\title{
MULTA SCRIPSIT, NIHIL TAMEN REPERITUR Niccolò Mattarelli giurista a Modena e Padova (1240 ca.-1314 ca.)
}

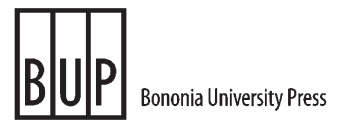


Bononia University Press

Via Ugo Foscolo 7, 40123 Bologna

tel. (+39) 051232882

fax $(+39) 051221019$

www.buponline.com

e-mail: info@buponline.com

(C) 2018 Bononia University Press

Tutti i diritti riservati

ISSN 2283-916X

ISBN 978-88-6923-301-2

Impaginazione: Sara Celia

Prima edizione: giugno 2018 
S E M I N A R I O G I U R I D I C O

D E L L A U N I V E R S I T À D I B O L O G N A

$* * *$

Per la valutazione della presente monografia la Giunta di Dipartimento ha nominato la seguente Commissione di lettura:

Prof.ssa Nicoletta Sarti (Alma Mater Studiorum Università di Bologna), per il Settore Scientifico Disciplinare IUS/19 Storia del Diritto medievale e moderno

Prof. Marco Cavina (Alma Mater Studiorum Università di Bologna), per il Settore Scientifico Disciplinare IUS/19 Storia del Diritto medievale e moderno

Prof.ssa Alessia Legnani Annichini (Alma Mater Studiorum Università di Bologna), per il Settore Scientifico Disciplinare IUS/19 Storia del Diritto medievale e moderno 

A Flavia, Laura e Simona insostituibili compagne di viaggio 



\section{INTRODUZIONE}

Nelle file dei doctores iuris, in specie civilisti, che la storiografia giuridica inquadra nella fluida e multiforme cornice dei postaccursiani $^{1}$ è senz'altro possibile riconoscere anche Niccolò Mattarelli, di origini modenesi ${ }^{2}$ e noto soprattutto come docente a Padova per un

1 Enrico Besta una novantina di anni or sono annoverava Mattarelli, anche se in forma cursoria, nella schiera dei postaccursiani avvicinandolo ai giuristi che «ancora maggior importanza ebbero quali commentatori»: BESTA, Fonti: legislazione e scienza giuridica, p. 825. Su tale feconda stagione del pensiero e della metodologia giuridica di età intermedia si veda CALASSO, Medio Evo del diritto, p. 545 ss.; NICOLINI, I giuristi postaccursiani; CoING, Handbuch der Quellen und Literatur, I, p. 268 ss., e p. 275 per una limitatissima bibliografia relativa a Nicolaus Matarellus, del quale si colloca erroneamente la morte nel 1339; Bellomo, Società e istituzioni in Italia, p. 445 ss. Si veda anche ID., Società e diritto, p. 307 ss., ove si sottolinea il ruolo creativo della dottrina postaccursiana, non appiattita nell'esclusiva funzione di predisporre ausilii e strumenti destinati ai pratici, e ID., Quaestiones in iure civili disputatae, p. XX, per la presa di distanza dalla lettura più tradizionale del «secolo racchiuso fra Accursio e Bartolo», segnato da una mediocre produzione scolastica destinata in primo luogo a causidici e pratici del diritto. In senso analogo anche le più recenti valutazioni di SARTI, Il diritto nei fatti, p. 461 ss., e anche ID., La città e lo studio nell'età di Pietro d'Anzola, p. 7, ove la categoria di «postaccursiani» è ritenuta espressione di «una storiografia ormai superata».

2 In forza di un incarico di insegnamento annuale a lui conferito nel giugno 1292, per il successivo anno scolastico a partire dalla data tradizionale del 29 settembre, dalla Universitas scolarium Cremone in iure civili allo scopo di leggere i libri del Codice, dell'Authenticum e i Tres Libri con apparato accursiano, si è talora pensato a Mattarelli come a un «grande giurista cremonese divenuto padovano»: in tal senso Bellomo, «Factum et ius», p. 88 e ID., Factum proponitur certum, p. 27; analoga valutazione in ID., I fatti e il diritto, p. 664. Per le fonti relative all'incari- 
ventennio negli anni a cavallo fra XIII e XIV secolo ${ }^{3}$. La figura di Mattarelli, la cui famiglia di giuristi Enrico Besta, con enfasi notevole e non senza esagerazione, collocava tra quelle di maggior spicco in età medievale per la propensione a trasmettere in via ereditaria la professione legale ${ }^{4}$, non appare certamente sconosciuta: a lui sono dedicati non pochi medaglioni biografici che, sin dal maturo Trecento, a pochi decenni dalla sua scomparsa, hanno progressivamente incrementato la conoscenza di fonti e notizie utili a delinearne il profilo giungendo sino ai nostri giorni. In proposito non è senza significato rimarcare che, assieme a Riccardo Malombra, è stato annoverato tra $\mathrm{i}$ «due maggiori giuristi padovani dei primi del Trecento» ${ }^{5}$ e che, anche in conseguenza di tale ruolo prestigioso, a lui sono dedicate voci specifiche nei più recenti repertori sia degli italiani in qualche modo illustri, sia specificamente dedicati al novero più ristretto dei giuristi ${ }^{6}$.

co didattico a Cremona si veda sotto, cap. I, nota 78. Mancano tuttavia conferme in merito all'accettazione da parte di Mattarelli e all'effettivo svolgimento dell'incarico.

3 La prima attestazione del legum doctor Niccolò Mattarelli a Padova risale al luglio 1290, l'ultima al luglio 1310: si veda cap. I.

4 Besta, Riccardo Malombra, p. 1; accanto a quella di Mattarelli sono ricordate le famiglie Accorsi, Denari, Belvisi, da Saliceto, Ubaldi e Socini. In proposito si ricorda che il padre di Niccolò, Giovannibuono, è attivo come notaio imperiale a Modena; un figlio di Niccolò, Francesco Mattarelli, è collega nel padre presso lo Studio padovano fra 1308 e 1310 e quindi giudice e vicario del podestà a Ravenna nel 1313-14; Giovannibuono (o Zambuono), altro figlio di Niccolò, insegna diritto canonico a Treviso dal 1314, a Padova nel 1326 ed è poi magister scholarum presso la Cattedrale di Modena; Antonio Mattarelli (...1306-1327...), figlio di Francesco, è ugualmente docente a Padova di diritto civile e nel 1336 viene eletto nel collegio degli statutari incaricati della riforma dei vigenti statuti modenesi risalenti al 1327: VicinI, Di Niccolò Mattarelli, p. 27 ss.; ID., Profilo storico, p. 19, nota 2.

5 Bellomo, Le istituzioni particolari e i problemi del potere, p. 213, nota 19.

6 Labardi, Mattarelli Niccolò; Semeraro, Niccolò Mattarelli. Non è ancora disponibile un catalogo aggiornato delle sue opere nel Compendium Auctorum Latinorum Medii Aevi (CALMA), organizzato su base alfabetica e giunto alla lettera G. Un limitato e parziale elenco delle opere attribuite a Mattarelli compare nel Repertorium fontium historiae medii aevi, 7, Romae 1997, p. 508. Mancando, allo stato delle conoscenze, autografi del giurista modenese, egli non viene annoverato nelle ricche sillogi curate da Giovanna Murano, Autographa, I.1. e Autographa, I.2. Un suo medaglione è assente anche dal recente repertorio dei docenti e degli studenti dell'ateneo patavino (Del Negro, Clariores), raccolta di brevi voci biografiche di taglio compilativo basate essenzialmente, per quanto concerne le figure dei giuristi, su quelle corrispondenti leggibili nel DBI, DBGI e nel Lexikon del Mittelalters. 
A fronte delle nebbie che hanno avvolto per secoli la produzione scritta di Mattarelli, lo spessore delle conoscenze su di lui ha subìto un incremento consistente unicamente dai primi del Novecento che ha interessato, con modalità e tempi differenziati, tanto l'aspetto più spiccatamente biografico quanto il profilo professionale documentato attraverso la sua produzione dottrinaria. Ancora alla metà dell'Ottocento Savigny non poteva che collezionare, in merito alla figura del giurista modenese, poche selezionate citazioni filtrate da illustri esponenti della scuola del Commento quali Cino e Giovanni d'Andrea, accanto a una breve serie di testimonianze tratte dall'erudizione bio-bibliografica soprattutto di età moderna. In ciò egli era largamente debitore delle raccolte documentarie tardo settecentesche realizzate in ambito padovano da Francesco Maria Colle e in ambito modenese da Girolamo Tiraboschi, il quale nel suo analitico repertorio degli scrittori di origine locale aveva tratteggiato il profilo di Mattarelli tramite una vasta rassegna di citazioni estratte dalla precedente letteratura tanto giuridica quanto, e più largamente, di impianto storico-erudito ${ }^{7}$.

All'alba del XX secolo Arrigo Solmi dava notizia, per la prima volta in modo circostanziato, di alcune opere di Mattarelli all'interno di codici vaticani e modenesi e ricordava la notevole serie di quaestiones citate da Alberico da Rosciate assieme ai compendi delle Letture odofrediane di cui aveva trasmesso il ricordo Guglielmo da Pastrengo verso la metà del Trecento. Sottolineando gli apprezzamenti tributati a Mattarelli anche da Cino, Giovanni d'Andrea, Bartolo e Baldo, Solmi ribadiva il contrasto tra la fama che avrebbe circondato il giurista modenese ai suoi tempi e l'oblio che ne aveva invece sepolto le opere, sulle quali, tranne minime eccezioni, «rimane l'oscurità più completa» ${ }^{8}$. Una prospettiva, quest'ultima, costan-

7 TiRaboschI, Biblioteca modenese, III, pp. 185-190. Al medesimo autore si deve un altro e più breve profilo di Mattarelli, non utilizzato da Savigny: ID., Storia della letteratura italiana, II, p. 346. Colle, Storia scientifico-letteraria dello Studio di Padova, II, pp. 77-84. Si tratta dei medaglioni più ricchi per segnalazioni di fonti risalenti e per l'approccio critico, in vari casi, alla loro lettura. Si rinvia al capitolo III per gli ulteriori profili biografici di Mattarelli prodotti nell'arco di vari secoli dalla storiografia sia di ambito giuridico, sia più latamente di carattere storico ed erudito.

8 Solmi, Alberto da Gandino, p. 169 s., nota 2, ove Mattarelli è ricordato come «giurista così famoso ai suoi tempi ed ora interamente ignorato nelle opere». 
temente ribadita da tutta la letteratura che a vario titolo e fin dal secolo XIV, a pochi decenni dalla sua scomparsa, si è occupata della sua figura e delle labili tracce, per certi aspetti incerte ed evanescenti, che dei suoi scritti parevano essersi conservate.

Nel medesimo torno di anni anche la documentazione relativa al giurista veniva messa in luce nel contesto di uno specifico e ampio inquadramento genealogico-famigliare, contribuendo a illuminare sia la dimensione privata del professionista e tecnico del diritto nell'ambito, in particolare, della sua città natale, sia diversi aspetti del suo impegno pubblico nelle file della parte popolare e della sua attività professionale in relazione soprattutto agli arbitrati che gli vengono affidati per risolvere numerose controversie private, agli incarichi di docenza a Modena e a Cremona e a quello di giudice a Foligno, ricoperto da Mattarelli nel 1282. Tale scavo archivistico si deve a Emilio Paolo Vicini (1872-1946), il miglior interprete della storiografia medievistica modenese del XX secolo di ambito non accademico, in grado di coniugare cultura giuridica (era laureato in giurisprudenza) e competenze paleografico-diplomatistiche (diresse l'Archivio Storico Comunale e, a lungo, l'Archivio Notarile distrettuale di Modena) applicandole a una seria opera di edizioni di fonti scritte e di approfondite ricerche, su fronti molteplici ed entro un orizzonte non esclusivamente locale ${ }^{9}$.

Le uniche note al giovane storico del diritto agli inizi del Novecento erano la repetitio copiata nel codice estense lat. 1161, certamente conosciuta grazie all'attività prestata a Modena sia come bibliotecario all'Estense nel 1898 sia come libero docente di Storia del diritto italiano dall'anno successivo (Mattone, Solmi Arrigo, p. 1889), e il Tractatus super instrumentis, leggibile all'interno del codice Vaticano latino 5773. Forse per il condizionamento indotto da una tradizione scritta lacunosa e dall'assenza di opere a stampa, ancora oggi si è portanti a ritenere che, eccetto quest'ultima opera, «niente si è salvato autonomamente» della sua produzione scritta: MatTarell, Tractatus super instrumentis, p. 8.

9 Con riferimento allo studio di VICINI, Di Nicolò Mattarelli, che verrà largamente citato in seguito e dal quale dipende in toto il sintetico medaglione biografico leggibile in Mor-Di Pietro, Storia dell'Università di Modena, I, p. 273. Più di recente in ambito locale si segnala il saggio di Bedoni, Niccolò Mattarelli, che tenta una esplorazione maggiormente documentata del profilo professionale del Maestro e della sua produzione, per quanto ancora in misura parziale e con imprecisioni nell'individuazione di alcune sue opere manoscritte. Per la figura e l'opera di Vicini si rinvia ai profili tracciati da Sorbelli, Emilio Paolo Vicini; ID., In memoria di Emilio Paolo Vicini. 
In tempi più prossimi a noi, dagli anni Sessanta del Novecento, una ricerca più mirata all'interno dei giacimenti vaticani ha portato all'affioramento di alcuni primi testi in seguito all'impegno di Mario Bevilacqua e ha soprattutto consentito di focalizzare l'attenzione sulla cospicua produzione di quaestiones attribuite a Mattarelli grazie all'avvio, da parte di Manlio Bellomo, di una prolungata serie di studi su due grandi e ora notissimi Libri Magni (Archivio San Pietro A.29 e Chigi E.VIII.245), completata nel tempo dal confronto e dall'integrazione con ulteriori cinque manoscritti individuati in altre biblioteche italiane ed europee ${ }^{10}$.

Da allora la conoscenza di opere riferite al giurista modenese si è notevolmente arricchita sia dal punto di vista quantitativo che tipologico: sermones pronunciati in occasione di esami di laurea; repetitiones a leggi del Codice e del Digesto vecchio; frammentarie testimonianze della sua attività didattica reportatae nei margini di codici giuridici legati a una circolazione in area padovana ma oggi dispersi in varie biblioteche su scala europea; trattazioni monografiche che sembrano procedere sia da scelte a libera discrezione del maestro sia da lecturae scolastiche. Tra esse, le più ampie e organiche vengono dedicate, in riferimento al primo gruppo, alla classificazione delle scritture pubbliche e private e alla loro forza probatoria e, relativamente al secondo, al valore della consuetudine e del diritto non scritto. Dall'impegno didattico di Mattarelli discende pure la redazione di Letture, in specie al Codice, di un apparatus e di un cursus lectionum - secondo le precise testimonianze di Baldo - ai quali si affianca il sunto delle Letture di Odofredo ricordato sempre all'altezza del secolo XIV da Guglielmo da Pastrengo con il nome di Decisa.

In merito all'attività di insegnamento una testimonianza rilevante è offerta dal codice 941 conservato alla Biblioteca Universitaria di Padova, un notissimo esemplare del Digesto vecchio già famigliare a Mommsen e a Besta ${ }^{11}$, databile alla prima metà del secolo XII ma

10 SARTI, Il diritto nei fatti, p. 457 s. Precisi riferimenti ai testi di Mattarelli e agli studi di Manlio Bellomo seguiranno nello sviluppo del testo e delle note.

11 Di Theodor Mommsen si veda la Praefatio all'edizione critica del Digesto, p. XXXXVIII s.; Besta, L'opera di Irnerio, p. IX ss. Dell'esame più approfondito del codice e delle ragioni per cui si suppone il suo utilizzo prevalente in ambito emiliano e padovano si dà conto nel cap. II. Sul verso del foglio di guardia una mano co- 
dalla lunga vita scolastica e per questo tormentato da una fitta trama di notazioni interlineari e marginali riconducibili a strati e mani diverse. Tra esse si contano numerose glosse e additiones chiuse dalla sigla $n y$, talora rafforzate da un'attenzione dell'estensore a evidenziare in modo puntuale e diretto le opzioni del maestro che sembra rivelare l'utilizzo del manoscritto, circolante dapprima in area emiliana e quindi padovana, direttamente all'interno della sua scuola.

Accanto al ventaglio di reportationes di derivazione scolastica e di testi dotati di sufficiente omogeneità tematica, a partire dal ricco serbatoio di quaestiones attribuite al docente modenese, si può cogliere la latitudine della sua produzione scientifica grazie anche ai folti richiami alle sue opinioni che emergono nella posteriore dottrina civilistica, soprattutto trecentesca, non soltanto come citazioni puntiformi allegate in appoggio o in contrasto alla linea argomentativa seguita dai rispettivi autori, ma in non pochi casi arricchite da testimonianze più approfondite del suo pensiero che ne chiariscono lo sviluppo logico e le opzioni interpretative.

Considerata in una prospettiva di lungo periodo, la memoria della figura e delle opere di Mattarelli potrebbe essere accostata con tutta la prudenza del caso - a quella del ben noto Pepo: tramandata in fonti coeve di XI secolo ma pure in scritti posteriori, ricomposta in modo frammentario in un profilo che appare incerto, per taluni aspetti, e non meno controverso, l'identità di quest'ultimo rimane condizionata, con minime eccezioni, dall'assenza di testimonianze scritte della sua riflessione in ambito giuridico ${ }^{12}$. Similmente, anche gli esemplari della produzione del maestro modenese scompaiono dal circuito della conoscenza diretta posteriormente al Trecen-

eva attesta che «Il presente codice manoscritto segnato col n. 941 fu dato a prestito per quattro mesi al Prof. presso l'Università di Berlino Dr. Mommsen, dietro interessamento (15 novembre 1864) della R. Legazione di Prussia a Vienna».

12 Eccezioni - come ben noto - relative a un parere in materia di mutuo tramandato in una summa Institutionum provenzale copiata verso la fine del XII secolo e a un intervento a sostegno della formulazione del verdetto in occasione di un placito celebrato in Lombardia verso il 1084 in presenza dell'imperatore: le fonti conosciute relative a Pepo sono ampiamente esaminate in Dolcins, Pepo, Irnerio, Graziano; ID., Velut aurora surgente; Struve, Die Salier und das römische Recht, p. 37, nota 112; Contese, Il diritto nella storia medievale, II, p. 57 ss.; Padovani, Alle origini dell'Università di Bologna, p. 13 s.; CORTESe, Pepo. 
to, mentre le informazioni cristallizzatesi nella successiva letteratura di carattere storico-giuridico e bio-bibliografico prodotta fra Quattro e Settecento, oltre a lamentare concordemente l'assenza di sue opere date alle stampe, vengono a incorporare dati molto parziali e fortemente imprecisi.

A fronte di questo quadro complessivo ancora limitato, per molti aspetti, e sbilanciato nella conoscenza dei diversi ambiti in cui si è sviluppata l'esperienza umana e professionale di Niccolò Mattarelli, questa ricerca è stata orientata su molteplici livelli, a partire dalla lettura delle fonti utili alla ricostruzione dettagliata del suo profilo biografico-professionale. Fonti che molto devono, per la parte maggiormente legata alle vicende private e famigliari del giurista, alle registrazioni presenti nei Memoriali notarili modenesi, che si rivelano di particolare utilità anche per rintracciare notizie sulla committenza di manoscritti giuridici, e non soltanto, da parte di Mattarelli e sugli incarichi professionali a lui conferiti - come già ricordato - quale docente a Modena e Cremona e giudice a Foligno.

In merito a tipologie e caratteristiche della produzione dottrinaria del maestro modenese si sono rintracciate tutte le testimonianze manoscritte a oggi note, sia frammentarie sia rispecchiate in testi più organici, con l'obiettivo di formare un primo inventario degli scritti a lui attribuiti grazie alla loro individuazione all'interno di un ventaglio copioso di codici italiani e stranieri ${ }^{13}$. A questi si affiancano ulteriori serie di allegazioni e rinvii a opinioni e scritti di Mattarelli diffusi nelle opere di giuristi, a lui pressoché contemporanei, quali in primo luogo Cino (1270 ca.-1336) ${ }^{14}$ e Giovanni D'Andrea (1271 ca.-1348), docente a Padova tra il tardo 1306 e l'autunno del 1309 e ancora nell'estate del $1319^{15}$, e anche seriori, ma che non sembrano oltrepassare la soglia degli inizi del Quattrocento per quanto con-

13 L'esigenza è già stata rilevata implicitamente da Murano, Copisti a Bologna, p. 31 s., nota 105, laddove, oltre a osservare che «del suo insegnamento (a Modena, Cremona e Padova) e della sua attività di giureconsulto rimangono sparse tracce», sottolinea che «non possediamo ancora il catalogo delle sue opere».

14 Per i dati relativi al profilo biografico si rinvia ai contributi più recenti di Maffer, Cino Sinibuldi da Pistoia, e di Murano, Cino da Pistoia, ove si rivolge specifica attenzione alle testimonianze manoscritte della sua produzione dottrinaria.

15 Bartocci, Giovanni d'Andrea, p. 1009; Autographa, I.1., pp. 44-50 (voce curata da G. Murano). 
cerne la conoscenza diretta di sue opere e la possibilità di attingere a esse.

Da quell'altezza cronologica si apre il largo spazio della produzione di testi classificabili nelle variegate categorie della storia giuridica e della bibliografia con ambizioni universali, entrambe destinate a rapida e larga diffusione, soprattutto in area italica e germanica, grazie al formidabile volano della stampa. Un'ampia rassegna di queste viene utilizzata nel terzo capitolo per ricostruire i percorsi seguiti dalla memoria postuma del giurista, nella quale si concentrano e si amplificano progressivamente notizie che è necessario sottoporre a confronti e valutazioni critiche per verificarne attendibilità e portata. Esse si rivelano comunque utili per osservare le forme e le modalità con cui si è conservato e diffuso, nello spazio e nel tempo, il ricordo del giurista modenese, delle tappe salienti della sua attività di docente e delle labili tracce delle sue opere sino al recupero più corposo e meditato cui si giunge sullo scorcio del Settecento per opera di Girolamo Tiraboschi. Grazie a una ricerca maggiormente documentata, al vaglio critico delle fonti e all'applicazione di un metodo storico che proprio in area modenese-estense affondava direttamente le radici nella lezione muratoriana.

Infine, a conclusione del lavoro e nella consapevolezza che esso non rappresenta che un avvicinamento parziale alla conoscenza dell'opera complessiva di Niccolò Mattarelli e del suo impatto sulla posteriore dottrina, in specie quella elaborata nel secolo immediatamente successivo alla sua scomparsa, desidero esprimere un particolare ringraziamento ad Andrea Padovani e Nicoletta Sarti, che hanno seguito la ricerca in tutte le sue tappe e dei cui numerosi e provvidi consigli essa si è largamente giovata. Un pensiero particolarmente grato anche a Marco Cavina ed Elio Tavilla, per le indicazioni metodologiche e i frequenti sproni alla concentrazione su questo studio in funzione della sua organica conclusione. Suggerimenti bibliografici e utili indicazioni devo a Bernardo Pieri, Ugo Bruschi e Riccardo Parmeggiani e ringrazio vivamente gli operatori di biblioteche e archivi italiani e stranieri che hanno generosamente supportato la consultazione di opere edite e inedite e la riproduzione di manoscritti. Rimangono a totale carico dell'autore imprecisioni e limiti, dovuti anche ai confini assai larghi e diversificati dati alla ricerca e al profilo di sintesi complessiva che essa si è posta sin dall'origine. 


\section{CAPITOLO I \\ CLARUS JURIS CAESAREI PROFESSOR \\ TAPPE DI UNA BIOGRAFIA PROFESSIONALE E POLITICA}

Sommario: 1. Le origini famigliari - 2. La matricola dei giudici e avvocati e la matricola dei notai -3 . La formazione giuridica: verso una 'linea alternativa' -4 . Lo Studio modenese nel Duecento e il primo magistero di Mattarelli - 5. Denaro, case, terre -6 . La committenza di manoscritti -7 . Al governo del Comune 8. Ancora nelle file del Popolo - 9. Il ritorno a Padova.

\section{Le origini famigliari}

Stando alle numerose testimonianze documentarie che rivelano considerevoli aspetti della sua vita privata e famigliare nonché di quella pubblica, Niccolò Mattarelli nasce, verosimilmente a Modena, nel corso degli anni Quaranta del secolo XIII. Proprio la quantità notevole di informazioni che connettono a questa città la rete delle sue relazioni personali, la dotazione patrimoniale da lui accumulata nel tempo, la sua identità professionale, oltre alla partecipazione alla vita delle locali istituzioni comunali in fasi decisive della loro evoluzione fra Due e Trecento, non può che convergere nell'identificare il giurista come di origini modenesi, sgombrando il campo da ipotesi emerse nel passato, e talora riaffiorate in tempi recenti, di possibili origini cremonesi oppure, in via ancora più remota, ravennati ${ }^{1}$.

Le fonti disponibili non permettono di acquisire informazioni

1 Per la discussione di tali ipotesi si veda qui di seguito e anche il cap. III. 
abbondanti sulle origini famigliari di Niccolò Mattarelli, il cui cognome, regolarmente utilizzato anche dai fratelli e dai discendenti diretti e collaterali, sembra derivare dal soprannome già portato dal padre, persona autorevole e nota nel panorama cittadino modenese dei decenni centrali del Duecento sia per l'esercizio della professione notarile, sia per il legame che, anche attraverso di essa, lo poneva in stretta relazione con l'autorità comunale.

Nel corso del 1243 il notaio imperiale Ioannes Bonus Boconus qui dicor [sic] Mattarelli è deputato alla scrittura degli atti del Consiglio Generale del Comune di Modena e, in specie, si occupa della registrazione di alcune deliberazioni inerenti la risoluzione di una controversia tra il Comune e il monastero femminile urbano di S. Eufemia $^{2}$ scaturita dal danneggiamento di un mulino di proprietà del monastero in seguito al prosciugamento di un'area paludosa estesa a sud della città fuori porta Baggiovara ${ }^{3}$. Ed è, probabilmente, in conseguenza dei vincoli parentali con i più stretti consanguinei del padre Giovannibuono Boconi, che una trentina di anni più tardi, il 5 agosto 1272, alla redazione del testamento di Richelda filia olim Raynerii Bochoni et nunc uxor Fosaregi ferarii interviene come testimone Nicholao de Matarello, già all'epoca stabilmente qualificato con il titolo di legum doctor ${ }^{4}$.

2 Sul monastero di Sant'Eufemia, documentato per la prima volta nel gennaio 1071 sotto l'episcopato del vescovo Eriberto ma legato, nelle proprie origini, a un ospizio funzionante a Modena già nel 1029, si rinvia ai dettagliati profili di Soli, $L a$ chiesa e il monastero di S. Eufemia, e di Al KalaK, Il monastero di Sant'Eufemia.

3 VICINI, Le pergamene del Monastero di S. Eufemia, n. 12, p. 10 (1243 giugno 15; Ego Iohannesbonus Boconus imperiali auctoritate notarius et tunc ad consilia scribenda pro comuni Mutine spetialiter deputatus); n. 13, p. 11 (1243 agosto 8; Ego Iohannesbonus Boconus notarius ad conscilia scribenda pro comuni Mutine spetialiter deputatus); n. 14, p. 12 (1243 novembre 9; Ego Iohannes Bonus Boconus qui dicor Mattarelli notarius ad consilia scribenda pro comuni Mutine spetialiter deputatus); $\mathrm{n}$. 15, p. 13 (1243 dicembre 11; Ego Iohannes Bonus Boconus qui dicor Mattarelli notarius ad consilia scribenda pro comuni Mutine spetialiter deputatus); n. 16, p. 13 (1243 dicembre 15; Ego Iohannes Bonus Boconus qui dicor Mattarelli imperialis aule notari$u s)$. Con riferimento alla prima di questa serie di scritture, già CAMPORI, Del governo a comune in Modena, p. CCXIX, nota 1, osservava come il cognome Mattarelli non fosse quello originario della famiglia del giurista, ma derivi dal soprannome portato dal padre di Niccolò. Concorda pure SANTINI, Università e società a Modena, p. 352.

4 ASMo, Memoriale antico, a. 1272/1, n. 716, ricordato in VICINI, Di Nicolò Mattarelli, p. 7. A conferma di probabili legami di parentela, Richelda torna anche come nome di una delle due figlie di Mattarelli. 
Tornando al padre di Niccolò, il 6 settembre 1259 Iohannebono Mattarelli, trovandosi in palatio novo comunis Mutine, testimonia all'accordo stipulato dal Comune con il vescovo Alberto Boschetti avente a oggetto la manutenzione e la pulizia del Canalchiaro, uno dei principali corsi d'acqua che attraversavano a cielo aperto la città ${ }^{5}$. Oltre a lui, sempre chiamati a testimoniare all'atto che coinvolge le maggiori istituzioni civili ed ecclesiastiche modenesi, sono ricordati Niccolò Bruneta, notaio del podestà, al fianco di alcuni esponenti di rilevanti famiglie dell'aristocrazia urbana e rurale. Accanto a essi, oggetto di un deferente e particolare riguardo che si riflette nella posizione di preminenza iniziale nella serie dei testimoni, compare il iuris professor Martino da Fano, il quale, dopo avere insegnato ad Arezzo fino al 1255 ed essere stato chiamato a Reggio a dirimere una controversia con il Comune di Bologna, tiene cattedra a Modena per alcuni anni sino al momento di assumere la podesteria di Genova nel $1260^{6}$.

Ancora nel settembre 1259 Iohannebono Mattarelli testimonia alla deliberazione del Consiglio generale degli Anziani vòlta a cassare una recente addizione agli statuti cittadini, secondo la quale le offerte tam de cereis quam de pallio donate alla chiesa cattedrale in occasione della festa del Santo patrono sarebbero dovute rimanere

5 RPCM, II, n. 329, a p. 207. Sul Canalchiaro, uno dei maggiori canali urbani in grado di assicurare il rifornimento di acque pulite originate da sorgenti poste a sud della città, si rinvia a BARACCHI-MANICARDI, Modena: quando c'erano i canali, p. 54 ss. Si rileva che Vicins, Di Niccolò Mattarelli, p. 7, verosimilmente per errori di trascrizione dal codice manoscritto, afferma che il notaio del podestà sarebbe il medesimo Giovannibuono e che nel codice, conservato in ASCMo, Camera Segreta, I.1, l'atto qui richiamato sarebbe contrassegnato dal numero progressivo 354 , mentre si tratta del n. 336 .

6 L'ampia bibliografia sul giurista marchigiano è ricordata in dettaglio nelle due voci biografiche di Liotta, Del Cassero Martino, e di Semeraro, Martino del Cassero da Fano. Un profilo biografico fortemente compendiato anche in LiotTA, Martino da Fano giurista e pratico del diritto. Sul punto del soggiorno di Martino a Reggio e a Modena si veda pure TAVILLA, Homo alterius, p. 201 s. Sulla sua esperienza di docente ad Arezzo e sull'incarico podestarile svolto una sola volta a Genova nel 1260, giacché nel 1262 il medesimo incarico viene conferito al figlio di Martino, Palmerio, si vedano, in particolare, le ricerche di NARDI, Martino da Fano, e di Petti Balbi, Martino da Fano, podestà a Genova. Sulla produzione giovanile del maestro fanese, con attenzione soprattutto alla materia processualistica filtrata attraverso i modelli documentari concepiti a uso dei notai, si ricorda l'analisi specifica di Padoa Schioppa, Martino da Fano processualista. 
presso la sagrestia del Duomo anziché andare a beneficio del massaro e della Fabbrica, come stabilito dal Capitolo della Cattedrale e dal vescovo Alberto già dal 1244 e confermato l'anno successivo da papa Innocenzo IV ${ }^{7}$.

Nello stesso torno di tempo il notaio imperiale Iohannesbonus Matarellus, dichiarando lo specifico titolo professionale nell'esercizio delle proprie funzioni, redige la deliberazione del Consiglio generale del Comune con cui si ratifica quella formulata dal Consiglio generale degli Anziani il giorno precedente, dopo avere verbalizzato alcuni mesi prima un'altra deliberazione, analoga per contenuto, con cui il Consiglio generale del Comune aveva già confermato alla Fabbrica di S. Geminiano la donazione delle oblazioni conferite al Santo in occasione della festa solenne del 31 gennaio e della vigilia, conformemente a quanto disposto dal Capitolo della Cattedrale e confermato dal pontefice ${ }^{8}$.

L'atto del 6 settembre 1259 sopra ricordato è incluso in entrambi gli esemplari dei poderosi Libri iurium compilati a Modena nella seconda metà del Duecento, il Registrum Privilegiorum e il Registrum Antiquum ${ }^{9}$, due importanti cartulari nei quali si riflettono le

7 Bonacini, La Fabbrica di San Geminiano, reg. 18, p. 66 (1259 settembre 4); reg. 1, p. 57 (1244 novembre 12); reg. 6, p. 59 (1245 marzo 29), nell'ordine.

8 Ibidem, reg. 19, p. 66, 1259 settembre 5; reg. 17, p. 65, 1259 marzo 25, nell'ordine.

9 ASCMo, Camera Segreta, I.1 e I.2. Cfr. LucchI, "Camera Segreta”, p. 11 s., con le correzioni di Pincella, Una signoria in crisi, p. 65 ss. Il primo tra i due codici, formato da 230 carte suddivise in 29 quaderni, contiene secondo la sua numerazione 343 atti datati dall'anno 969 al 1260, mentre il secondo, costituito da 291 carte di maggiore formato, contiene 702 atti numerati e datati sempre dall'anno 969 sino al 1315, di cui i primi 328 risultano inclusi pure nel Registrum Privilegiorum, Parte degli atti riportati in quest'ultimo, sino all'anno 1218 , sono esemplati dal notaio Degoldeo, il quale, con precisa regolarità, si sofferma ad autenticare ciascuno di essi, mentre la prima sezione del Registrum Antiquum risulta copiata nel 1263 dal notaio Delayto, che inserisce la propria formula di autenticazione soltanto alla fine del lavoro complessivo. La mancanza, in quest'ultimo codice, di una serie iniziale di atti presenti invece nel Registrum Privilegiorum è stata determinata dall'asportazione delle prime 30 carte del Registrum Antiquum, che nel suo complesso rimane tuttora inedito Il Registrum Privilegiorum è invece pubblicato in RPCM I-II, ove si veda in particolare l'Introduzione nel vol. I. La struttura e le modalità compositive di questo codice sono state esaminate più di recente e in modo approfondito da RöLKER, Il Registrum privilegiorum del Comune di Modena. Allo stesso autore si rinvia per un ulteriore studio specifico del Registrum Antiquum e dei suoi complessi rapporti compositivi con 
tipologie documentali tipiche dei Libri Iurium comunali italiani: diplomi imperiali o regi, patti e convenzioni tra comuni, atti di sottomissione compiuti da signori laici o ecclesiastici oppure da comunità del territorio, investiture feudali, cittadinatici, acquisizioni patrimoniali da parte del comune, locazione di beni immobili, quietanze di pagamento. Tutto il complesso, insomma, dei diritti, delle proprietà e delle prerogative accumulate nel tempo dall'ente comunale a partire dalle più antiche testimonianze che ne costituiscono la garanzia legittimante, filtrato attraverso una cosciente operazione di selezione e ordinamento dei singoli atti finalizzata al loro inserimento in apposite raccolte che vedono la luce, a seconda dei vari contesti cittadini, a partire dagli ultimi anni del secolo XII e poi lungo il corso del Duecento ${ }^{10}$.

il Registrum Privilegiorum in corso di stampa negli «Atti e Memorie dell'Accademia Nazionale di Scienze Lettere e Arti di Modena».

10 Per un panorama d'insieme in merito alle ricerche sui Libri Iurium dei comuni italiani, con analisi tanto sulla genesi formativa di tali raccolte quanto sulla fisionomia della documentazione in essi confluita, si vedano Rovere, $I$ «libri iurium» dell'Italia comunale; Cammarosano, Italia medievale, p. 144 ss.; Rovere, I «Libri iurium» delle città italiane; EAD., Tipologie documentali. Sulle scritture comunali e la loro genesi sono importanti le osservazioni, accompagnate da ricca bibliografia, di BAIETTO, Scritture e politica, nonché i saggi e l'Introduzione di AlBinI, Le scritture del comune. I Libri iurium - come noto - riuniscono gli atti scritti che documentano le ragioni formali della vita del Comune privilegiando quelli che meglio rispondono alla realtà politica contingente al momento della loro formazione ed escludendone a volte altri che, pur di notevole rilievo, per varie ragioni non producono più effetti giuridici, e in ciò seguendo una duplicità di criterio che pare costituire un fattore di distinzione notevole tra la prima e la seconda metà del secolo XIII, quando si afferma in molti casi un intervento ordinatore ben preciso e coerente nell'articolazione interna dei Libri Iurium teso a privilegiare una strutturazione tematica dei documenti in essi raccolti e a superare così la varietà di soluzioni compositive che aveva invece segnato i decenni precedenti. La ragione di questa differenziazione può risalire al quadro istituzionale dominante, distinguendo tra i libri della prima generazione, elaborati in epoca podestarile tra la fine del secolo XII e i primi decenni del Duecento, cui fa eccezione il precoce caso genovese (PUNCUH-Rovere, $I$ Libri Iurium, p. 19 ss.), e quelli più organici o monotematici della seconda generazione predisposti nella città, soprattutto settentrionali, dominate da contesti politici sia popolari che signorili (CAmmarosano, Prospettive di ricerca, p. 61), oppure si può connettere a prevalenti e maggiormente consapevoli esigenze di riorganizzazione e salvaguardia del patrimonio documentario posseduto dall'ente comunale, con la conseguente realizzazione di raccolte impostate secondo criteri tali da consentire un più facile recupero dei documenti al loro interno (su questo, in particolare, RoVERE, Tipologie documentali). 
Gli ulteriori atti in cui compare, in qualità di teste oppure di estensore, il notaio imperiale Giovannibuono Mattarelli sono invece inclusi nei due più antichi Libri iurium della Fabbrica o Fabbriceria del Duomo (secondo il termine ora di uso più frequente), l'istituzione che fin dal XII secolo, con la denominazione di domus (laborerii) sancti Geminiani e in connessione già con le prime fasi costruttive della Cattedrale cittadina e della torre contigua (l'odierna Ghirlandina), si è occupata del concreto svolgimento dei lavori edilizi, delle permanenti esigenze di integrazione, accrescimento e manutenzione dell'imponente complesso architettonico nonché del reperimento delle indispensabili risorse finanziarie necessarie alle varie fasi dei lavori grazie al sostegno precipuo, sotto il profilo economico-patrimoniale, del vescovo e delle autorità comunali.

Il codice più risalente tra essi (Capitolare O.III.30) è approntato dal notaio Bartolomeo Spadari di San Marco tra il 1259 e il 1261 riportando, in due sezioni distinte, gli statuta deliberati dal Comune di Modena in favore della Fabbrica di S. Geminiano tra il 1217 e il 1259 e quindi le concessioni e i privilegi elargiti o confermati a quest'ultima dal Comune di Modena, dal vescovo, dal Capitolo e dal papa tra il 1167 e lo stesso 1259. Un secondo e più completo $\mathrm{Li}$ ber della Fabbrica di S. Geminiano (codice Capitolare O.II.11) viene redatto dal notaio imperiale Niccolò Bruneta a partire dal 1271, verosimilmente su incarico del massaro della Fabbrica medesima, riordinando l'intera documentazione sino ad allora disponibile in base a uno schema ancora binario. In un primo gruppo di fascicoli è copiata una serie di atti già inclusi nel codice precedente e poi in un secondo gruppo tutti gli altri seguendo un ordinamento - a grandi linee - topografico, ossia riunendo assieme gli atti riferiti a beni collocati in uno stesso luogo o pertinenti a un medesimo nucleo patrimoniale. Negli anni successivi lo stesso notaio Bruneta procede a ulteriori integrazioni del lavoro già svolto copiando e autenticando altri atti nelle carte rimaste bianche all'interno dei fascicoli preesistenti e quindi redigendo cinque nuovi fascicoli con ulteriore documentazione dei secoli XII e XIII, assai eterogenea sia per cronologia che per contenuti ${ }^{11}$.

11 Su tutto questo Bonacini, Alle origini del codice O.II.11, pp. 10 ss., 20 ss. 
La documentazione, pur non abbondante, inerente l'attività di Giovannibuono Mattarelli ne mette in luce la qualifica professionale di notaio imperiale e l'attività svolta a stretto contatto con gli organi comunali tra gli anni Quaranta e Cinquanta del Duecento, quando partecipa sia a importanti accordi e deliberazioni assunte da questi ultimi, in qualità di testimone, sia direttamente alla redazione di deliberazioni del Consiglio generale del Comune. Come attesta una piccola lapide sepolcrale ancor oggi visibile su un pilastro della cripta del Duomo di Modena, il dominus Giovannibuono de Mattarellis muore il 29 novembre $1266^{12}$ e sei anni più tardi la sua scomparsa trova conferma nelle fonti scritte ${ }^{13}$.

\section{La matricola dei giudici e avvocati e la matricola dei notai}

La prima citazione conosciuta di Niccolò Mattarelli qualificato con il titolo di doctor legum risale al 1270 e compare nella redazione originaria della matricola dei giudici e degli avvocati di Modena, trascritta di seguito allo statuto duecentesco del medesimo Collegio. Il codice contenente lo statuto dei giudici e degli avvocati porta nell'intestazione il riferimento al 1337, l'anno successivo al rientro in Modena dei marchesi estensi dopo l'esaurimento della prima fase del loro dominio sulla città in seguito alla rivolta popolare scoppiata nel gennaio 1306, e si può ritenere copia fedele di quello approvato dal Collegio dei giudici - come si legge nel codice stesso - il 4

12 BeRTONI, Atlante storico-paleografico, scheda n. XVII, con trascrizione e riproduzione fotografica della lapide, che oggi risulta spostata rispetto alla sua collocazione originaria. Più recente trascrizione anche in Frugoni, Il Duomo di Modena. Testi, p. 389, n. 1366, con riproduzione fotografica in EAD., Il Duomo di Modena. Atlante, p. 707, n. 1366. La lapide è trascritta, in forma parziale e inesatta, pure in VICINI, Di Niccolò Mattarelli, p. 7.

13 ASMo, Memoriale antico, a. 1272/1, n. 2148: il 20 febbraio 1272 dominus Nycholaus legum doctor, magister Iohannes Novellus, Allexander fratres qd. Domini Iohannisboni Mattarelli vendono a Bernardo filio olim domini Petri de Suzo da Vignola un terreno arativo dell'estensione di 3 biolche ubicato super Formigine vetere per la somma di 27 lire modenesi. Il padre defunto di Niccolò Mattarelli e dei suoi fratelli è ricordato pure in altri atti riassunti nei registri dei Memoriali notarili citati nelle note successive. 
aprile 1270 in pallacio novo comunis Mutinae, che venne comunque preceduto da redazioni più antiche: due rubriche del medesimo statuto portano la data del 1262 e del 1267 e nella redazione trecentesca viene ricordato che il notaio Niccolò Bruneta lo estrasse ex autentico seu antiquo volumine statutorum collegii iudicum fideliter et dilligenter. La stessa redazione datata 1337 è peraltro anteriore a quell'anno, anche se non di molto, poiché il suo proemio venne scritto su evidente rasura allo scopo di adattare lo statuto alle nuove condizioni politiche indotte dal rinnovo della signoria estense sulla città risparmiando, nel contempo, la miniatura sovrastante collocata in apertura del testo e databile intorno al 1325, in sincronia con la scrittura di quest'ultimo ${ }^{14}$.

A margine della carta ove inizia la trascrizione dei nomi degli iscritti al Collegio dei giudici e avvocati il nome di Niccolò Mattarelli è affiancato da quelli, aggiornati nel tempo, dei suoi consanguinei Franciscus ac Thomas et Gentilis ac Diomedes, Antonius de Mattarellis omnes, nei quali si possono identificare il figlio di Niccolò, Francesco, e il figlio di questi, Antonio, entrambi doctores legum, oltre a Gentile, giurisperito e nipote di Alessandro, uno dei due fratelli di Niccolò. Più incerta invece l'identificazione di Diomede: nella genealogia approntata agli inizi del XX secolo da Emilio Paolo Vicini sono ricordati il giudice Diocles, nipote di Gian Novello, notaio, medico e fratello di Niccolò, e l'omonimo notaio figlio dello stesso Gian Novello e nipote ex fratre di Niccolò. Di Tommaso non si conoscono al momento tracce documentarie ${ }^{15}$.

Si hanno invece alcune notizie relative a Gian Novello, altro fratello di Niccolò e verosimilmente più anziano di lui: esercita la professione medica, nel 1276 rimane vedovo della consorte, Egidia de

14 ASCMo, Camera Segreta, IV.II.1, con edizione recente a cura di Valeria Braidi in Braidi-Tavilla, Statuti del Collegio dei giudici e degli avvocati, pp. 77-104, che include le integrazioni apportate successivamente al XIV secolo sia alla matricola, sia al codice nel suo complesso fino alla fine del Settecento. La citazione del dominus Nicolaus de Mattarellis doctor legum è a p. 85. Per criteri adottati e completezza testuale, tale edizione aggiorna quella precedente leggibile in VIcINI, Statuta iudicum et advocatorum, ove la citazione suddetta è a p. 24. Sul Collegio degli avvocati di Modena tra Medioevo ed Età Moderna si può vedere il sommario Della Fontana, Il collegio degli avvocati, benché privo di note e di apparato bibliografico.

15 VicinI, Di Nicolò Mattarelli, p. 31 s. e tavola genealogica finale. 
Poltoneriis, da lui sposata pochi anni prima ${ }^{16}$, e fa testamento il 19 gennaio 1292 morendo entro il mese successivo ${ }^{17}$. Ai sensi di una norma statutaria risalente alla metà del Duecento, egli è tra i destinatari di una serie di immunità ed esenzioni concesse dal Comune a una schiera numerosa di medici e cerusici, tutti dichiarati liberi e immuni da imposizioni e prestazioni comunali a contenuto tanto economico quanto materiale ${ }^{18}$. Nelle fonti scritte, tra 1272 e 1280 Gian Novello è di frequente attestato con il titolo di magister ${ }^{19}$, distintivo della professione da lui esercitata e forse da mettere in relazione con un'attività di insegnamento svolta in forma privata oppure presso la Scuola Capitolare cittadina, che emerge come «l'incubatrice da cui è nato lo 'Studium'» e nel cui ambito si ipotizza il funzionamento di una scuola medica almeno dal secondo quarto del secolo XII ${ }^{20}$.

Oltre che nella matricola tardoduecentesca dei giudici e avvocati di Modena, Niccolò Mattarelli è registrato anche in quella dei notai, di poco anteriore alla prima, assieme ad alcuni famigliari. Agli anni 1335-36 risale la redazione e l'entrata in vigore del primo statuto notarile modenese a noi giunto, cui venne unita la trascrizione della matricola degli associati realizzata tra la fine del 1347 e i primi mesi del $1348^{21}$ contenente, pur con alcune lacune ${ }^{22}$, i nomi de-

16 ASMo, Memoriale antico, a. 1271, n. 1571: il 20 aprile 1271 Gian Novello e Alessandro, a nome anche del fratello Niccolò, dichiarano di avere ricevuto da Bernardino de Poltoneriis la somma di 40 lire imperiali a titolo di dote per la figlia di questi, Egidia, promessa sposa a Gian Novello.

17 Vicini, Di Nicolò Mattarelli, p. 31; Bertoni-Vicini, I codici di un medico modenese, p. 12; VICINI, Medici modenesi nei secoli XIII e XIV, p. 28.

18 ID., Profilo storico, p. 23 e nota 3; ID., Medici modenesi nei secoli XIII e XIV, p. 5; BedonI, La medicina a Modena, p. 74.

19 ASMo, Memoriale antico, a. 1272/1, n. 2148; a. 1275, n. 2667; a. 1277, n. 4955; a. 1280, n. 3586; a. 1280, n. 4999.

20 SANTINI, Università e società nel XII secolo, p. 139; ID., Università e società a Modena, p. 330, da cui la citazione; Bedons, La medicina a Modena, p. 73.

21 Vicins, Di un codice di statuti notarili, pp. 6, 63. Nel codice manoscritto contenente lo Statuto, conservato presso l'Archivio Notarile di Modena, a f. 119v si ricorda che il 30 settembre 1336 i massari dell'Arte o Collegio dei notai Bonifacio Morano e Zanne de Sigonibus hanno consegnato il codice stesso al sacrestano della chiesa dei Frati Minori affinché vi sia conservato. Sul testo statutario e sul complesso della normativa in esso contenuta si veda BraIDI, Lo Statuto del Collegio dei notai, che integra e aggiorna il pur fondamentale studio di Emilio Paolo Vicini del 1910, in Appendice al quale viene pubblicata la Matricola sopra ricordata.

22 Bonacini, Scribarum turba Mutine, p. $52 \mathrm{~s}$. 
gli iscritti al Collegio notarile vissuti al tempo in cui furono redatti e applicati i primi Statuti alla metà degli anni Sessanta del Duecento; nomi che vengono elencati in base alla residenza nei rispettivi quartieri urbani, corrispondenti alle ripartizioni della città e del territorio circostante secondo le quattro principali porte cittadine (Albareto, S. Pietro, Baggiovara e Cittanova).

Nella Matricola non è registrato il notaio Giovanni Buono Mattarelli, il padre di Niccolò morto il 29 novembre 1266, per cui si può presumere che la sua redazione sia stata avviata successivamente a tale data. Diversamente, fra i notai già defunti residenti nel quartiere di porta S. Pietro già nella prima carta componente la Matricola è ricordato Niccolò Mattarelli, cui seguono più avanti il fratello Sander (Alessandro) e il figlio di questi, Corradino ${ }^{23}$, il nipote Dyocles, figlio del fratello medico Gian Novello ${ }^{24}$, e quindi Francesco, figlio dello stesso Niccolò ${ }^{25}$. In una carta successiva, sempre tra i notai defunti di porta S. Pietro, la Matricola riporta anche il nome di Attaleus de Mattarellis, altro figlio del medico Gian Novello e nipote di Niccolò ${ }^{26}$.

\section{La formazione giuridica: verso una 'linea alternativa'}

Tra la fine degli anni Sessanta e i primi anni Settanta del Duecento Mattarelli viene aggregato ai collegi professionali destinati a riunire e disciplinare quanti praticano le professioni legali in città e nel distretto. Nella matricola dei giudici e avvocati, composta nel

${ }^{23}$ VICINI, Di un codice di statuti notarili, pp. 26, 27 e 28; nel codice a f. 51vb e f. 52vb. Contando i nomi dei notai defunti a partire dall'inizio dell'elenco, quello di Mattarelli risulta il numero 105. Sul fratello del giurista, già defunto nel 1321, si veda il medaglione biografico di ID., Di Nicolò Mattarelli, p. 32 s. per Corrado/ Corradino, come viene ricordato nella Matricola dei notai, si veda l'albero genealogico in fine a quest'ultimo saggio.

24 ID., Di un codice di statuti notarili, p. 28; nel codice a f. 52va. Breve profilo del notaio Dyocles in ID., Di Nicolò Mattarelli, p. 31 s.

25 ID., Di un codice di statuti notarili, p. 28; nel codice a f. 52vb. Un profilo di Francesco, notaio e doctor legum, in ID., Di Nicolò Mattarelli, p. 27 s.

26 ID., Di un codice di statuti notarili, p. 30; nel codice a f. 54rb. Breve profilo di Attaleo, unito a quello del fratello Dyocles, in ID., Di Nicolò Mattarelli, p. 31 s. 
1270 a pochi anni di distanza da quella dei notai, egli risulta qualificato con il titolo di doctor legum e ciò induce a collocare in questo breve arco di tempo l'acquisizione, da parte sua, delle insegne dottorali. Un passaggio che trova conferma in un atto privato datato 20 febbraio 1272, il primo, sotto il profilo cronologico, in cui egli appare insignito del medesimo e prestigioso titolo ${ }^{27}$.

Un ulteriore elemento che concorre a circoscrivere il periodo cui fare risalire la nascita di Mattarelli è fornito ancora dallo statuto dei giudici e degli avvocati modenesi. Infatti, ai sensi del combinato disposto da una rubrica di questo datata 6 maggio 1267 e da un'altra contenuta nello statuto cittadino e richiamata all'interno della prima, all'esame per essere ammessi nella matricola del Collegio, basato sulla discussione di fronte a otto giudici collegiati di puncta individuati casualmente all'interno dei titoli del Digesto vecchio e del Codice, possono accedere i cittadini modenesi che abbiano studiato in legibus continue per quinquennium in scolis cum dominis legum, certificando tale preparazione attraverso testi idonei oppure tramite i rispettivi maestri, ai quali si deve prestar fede sine sacramento ${ }^{28}$.

Registrato pertanto nella matricola di giudici e avvocati nel 1270 con almeno cinque anni di studio giuridico alle spalle, già peraltro culminati nell'acquisizione del titolo di doctor legum, Niccolò Mattarelli assai verosimilmente può essere nato verso la metà degli anni Quaranta del Duecento e, di conseguenza, può avere studiato diritto a cavallo degli anni Cinquanta e Sessanta ${ }^{29}$, anche se manca-

27 ASMo, Memoriale Antico, a. 1272/1, n. 2148: si veda sopra, nota 13, e sotto, nota 80.

28 Braidi-Tavilla, Statuti del Collegio dei giudici e degli avvocati, rub. $<12>$, p. 83 s. e p. 27 , ove si richiama la rubrica poi confluita nella successiva redazione trecentesca degli statuti cittadini (Statuta civitatis Mutine, 1. I, r. CXXII, De offitio iudicum seu advocatorum Comunis, p. 98 ss.). Prerequisito per sostenere l'esame di ammissione è il possesso della cittadinanza modenese, riconosciuta dopo che il soggetto interessato vel familia sua abbia dimorato per 10 anni consecutivi in città oppure per 20 nel contado.

29 Risulta priva di fondamento la notizia secondo la quale Mattarelli sarebbe documentato come studente a Bologna nel 1269, riferita da GLORIA, Monumenti della Università di Padova, p. 249, sulla base di SARTI, De claris Archigymnasii Bononiensis professoribus, p. 234 ss., ove in realtà non compare il nome del giurista modenese. Per le imprecisioni e i limiti della prima edizione dell'opera di Mauro Sarti, affidata per la sua continuazione, purtroppo infarcita da rilevanti negligenze e omissioni, all'abate camaldolese Mauro Fattorini, si veda la Prefazione di Carlo Ma- 
no completamente notizie circa una sua formazione scolastica modenese nonché eventuali riferimenti all'opinione o alla grata memoria dei propri maestri all'interno delle sue opere, secondo una consuetudine tutt'altro che rara nella produzione dottrinaria dei giuristi di età intermedia.

In merito un indizio significativo affiora nella letteratura storico-genealogica già a breve distanza dalla scomparsa di Mattarelli, che tuttavia può prestarsi a una duplice lettura: da un lato condurrebbe a ipotizzare la frequentazione, da parte di Mattarelli, della Scuola bolognese del celebre Odofredo, attivo con continuità nella città felsinea fino alla morte, avvenuta sullo scorcio del 1265; dall'altro, si potrebbe spiegare come una consapevole opzione metodologica conseguente alla preparazione giuridica acquisita presso un Studium di minore caratura rispetto all'Alma Mater e più tipicamente legato, nel pieno del secolo XIII, allo sviluppo di interessi dottrinari diversificati e flessibili rispetto al rigido modello degli apparati di produzione azzoniana e accursiana ${ }^{30}$.

Entro l'orizzonte del secolo XIV, a pochi decenni dalla morte di Mattarelli, notizie importanti sulla sua produzione dottrinaria sono fornite da Guglielmo da Pastrengo, che già Savigny collocava pressoché alle origini della storiografia giuridica nella sua preziosa rassegna di autori rilevanti sia per la redazione di singole opere monografiche, sia per avere disseminato i propri testi, in misura più o meno intensa o cursoria, di notizie sulla vita e gli scritti di altri e più risalenti maestri del diritto di età intermedia ${ }^{31}$.

Guglielmo da Pastrengo è personaggio rilevante nella cultura padovana - e non soltanto - del pieno Trecento. Discepolo di Oldra-

lagola alla successiva edizione ottocentesca: SARTI-FATTORINI, De claris archigymnasii bononiensis professoribus, I, pp. 7-27.

30 La vicinanza di Mattarelli alle tipologie testuali e alle metodologie tipiche dei giuristi della seconda metà del Duecento che i numerosi studi di Manlio Bellomo individuano come esponenti di una "linea alternativa" rispetto a quella rappresentata dalla linea di sviluppo degli apparati di Azzone e Accursio sarà meglio specificata nel cap. II; si veda anche sotto, nota 44.

31 Savigny, Storia del diritto romano, I, p. 486 s., ove Guglielmo da Pastrengo è ricordato, in sequenza cronologica, subito dopo Giovanni d'Andrea nella serie di autori le cui notazioni o le cui opere risultano preziose per ricostruire il profilo biografico e dottrinario di numerosi giuristi di età intermedia. 
do da Lodi (1270 ca.-post 1345), si addottora nel prestigioso Studio veneto ed è avvocato, notaio e giudice in Verona, ove ricopre uffici pubblici di rilievo. Incaricato più volte di svolgere ambasciate per conto del papa in Avignone, è intimo amico e corrispondente di Petrarca partecipando, con raffinata competenza letteraria, a quella stagione della cultura dotta di matrice urbana e civile identificata come 'preumanesimo' padovano, le cui radici affondano già nel secolo XIII alimentate anche da fecondi contatti con lo Studio bolognese ${ }^{32}$.

Nel suo De viris illustribus, galleria di personaggi divenuti celebri per meriti acquisiti in vari campi dell'opera umana tra i quali non manca un discreto catalogo di giuristi corredato da non poche e circostanziate notizie e un altro di giureconsulti romani con l'indice delle rispettive opere, Guglielmo ricorda il Maestro modenese oltre che per il rudis eloquio, non troppo apprezzato al confronto di un più raffinato gusto espressivo di impronta già umanistica, anche per una ricca produzione di quaestiones e repetitiones e per avere compendiato - a uso assai verosimilmente didattico - le celebri Letture di Odofredo su Codice e Digesto in più snelli testi noti come Decisa, ottenuti cassando parti superflue, discorsive e certamente accessorie rispetto al cuore della riflessione giuridica per enucleare da quest'ultima - come si può supporre - il distillato del precetto interpretativo dell'illustre maestro felsineo ${ }^{33}$.

32 Billanovich, Il preumanesimo padovano; PolizzI, Nuovi documenti e ricerche sul cenacolo preumanistico padovano; BILlanovich, I primi umanisti padovani; CONETTI, La dottrina dell'impero, p. 354 s. con riferimento particolare agli anni della formazione universitaria di Alberico da Rosciate e alla sua complessiva formazione culturale di taglio non esclusivamente giuridico. Per un dettagliato profilo dell'autore padovano e delle sue opere, composte verso la metà del Trecento e trádite in 5 testimoni (4 manoscritti non autografi, di cui uno risalente al sec. XVIII, e una versione a stampa apparsa a Venezia nel 1547), si veda la robusta Introduzione in Guglielmo da Pastrengo, De viris illustribus et De originibus, pp. IX-XCIV, assieme al più recente contributo di Cerroni, Guglielmo da Pastrengo. Vedi pure SERraI, Storia della bibliografia, I, pp. 261-63 e AvESANI, Il preumanesimo veronese, p. 126 ss.

33 Guglielmo da Pastrengo, De viris illustribus et De originibus, p. 165: «Nicolaus de Macarelis de Muttina, legum doctor, scientia clarus sed eloquio rudis, Odofredi Lecturam, quam super Digesto et Codice exposuerat amotis supervacuis decidit, quod opus Decisa nuncupavit; Quaestiones bonas et utiles disputavit, quas cum legum multarum repetitionibus scripto traditis volentibus dereliquit». Si veda anche il più sintetico profilo di Odofredo: «Odofredus Bononiensis, legum doctor 
Vissuto tra la fine del Duecento e il 1362, quindi di un paio di generazioni più giovane di Mattarelli qui fuit magnus homo in iu$r e^{34}$, Guglielmo da Pastrengo è tra i testimoni a lui più prossimi poco più giovane di maestri quasi contemporanei a Mattarelli come Cino o Giovanni D'Andrea - sia per contiguità cronologica che per la sua origine padovana e per lo studio svolto alla scuola di Oldrado, collega a Padova di Mattarelli, almeno dal 1305 e maestro, tra gli altri, di Alberico da Rosciate ${ }^{35}$.

Pure ad Alberico (1290 ca.-1360), coetaneo del Pastrengo e cresciuto scolasticamente nell'ambiente padovano come allievo, oltre che del maestro lodigiano, anche del cremonese Riccardo Malombra $^{36}$, non risultano ignoti i compendi di Mattarelli alle Letture odo-

eximius, super Digesto veteri et Codice Lecturas egregias edidit, opus docentibus, discentibus et doctis utilissimus» (p. 170), ove si rimarca l'utilità delle Letture predisposte dal Maestro felsineo non soltanto per gli studenti e i tecnici del settore, ma anche per gli stessi colleghi, come ribadito nell'esempio di Mattarelli. Con modeste varianti si veda pure [Guglielmo Da PASTRENGo] De originibus rerum libellus authore Gulielmo Pastregico [...], Venetiis 1547, f. 52v: «Nicolaus de Ma<ta>relis de Muttina Legum Doctor scientia clarus, sed eloquio rudus Odofredi lecturam quam super Digesto et Codice exposuerat a montis supervacuis decidit, quod opus decisa nuncupavit. Questiones bonas et utiles disputavit, quas cum legum multarum repetitionibus scripto traditis volentibus dereliquit». Operazione ben diversa è compiuta dal fiorentino Bonaccorso degli Elisei, che ripete gli insegnamenti dei propri maestri bolognesi, Odofredo e il figlio Alberto, cogliendoli dalla loro viva voce e condensandoli all'interno di un trattato di argomento processuale composto forse nei primi anni Settanta del secolo XIII: MAfFeI, Un trattato di Bonaccorso degli Elisei, in part. p. 33; SoEtermeer, Une catégorie de commentaires peu connue, p. 49 ss.

34 Apprezzamento apertamente dichiarato nei confronti di Mattarelli in Oldrado da Ponte, Consilia, cons. 88, Tertio quaeritur de tali quaestione, f. 33ra, n. 1.

35 Per tali dati, oltre che per un approfondimento della biografia e della produzione scientifica del maestro lodigiano, si rinvia agli studi di Chiara Valsecchi, con completa bibliografia anteriore: VALSECCHI, Un'auctoritas del primo Trecento. Oldrado da Ponte, in specie p. 11 ss.; EAD., Oldrado da Ponte (a); EAD., Oldrado da Ponte (b). Vedi pure Guglielmo da Pastrengo, De viris illustribus et De originibus, p. XVII s. Durante il periodo di comune insegnamento a Padova Mattarelli compare al fianco di Oldrado in alcuni diplomi di laurea risalenti agli anni 13081310: Gloria, Monumenti della Università di Padova, p. 65 ss. e p. 259 s. per l'insegnamento patavino di Oldrado.

36 Sul profilo biografico e dottrinario di Alberico e sulla sua ricca produzione, di ambito non esclusivamente giuridico, sono importanti SAvignY, Storia del diritto romano, II, p. 625 ss.; ProsDocimI, Alberico da Rosciate e la giurisprudenza italiana del secolo XIV; CremaschI, Contributo alla biografia di Alberico da Rosciate; 
frediane, saltuari riferimenti ai quali emergono tanto nel Commentario al Codice ${ }^{37}$, quanto nella sua notissima opera dedicata alla materia statutaria ${ }^{38}$. Di essi, tuttavia, come opere autonome non viene fatta menzione nei testamenti dettati dal giurista bergamasco, nelle sezioni dedicate alla precisa e copiosa rassegna dei libri da lui posseduti, distinti con rigore - all'interno del testamento più risalente tra libri di diritto civile (elencati in due gruppi), libri extraordinarii («trattati di retorica, esempi di composizioni di discorsi, formulari giuridici, opere di letteratura, di storia o mitologia, e alcune altre varie»), libri di diritto canonico, libri morales (a prevalente contenuto filosofico e letterario) e, infine, di medicina ${ }^{39}$.

Le precoci testimonianze di Guglielmo da Pastrengo e di Alberico da Rosciate convergono nell'attestare l'opera perduta del giurista modenese, nella quale pare rivelarsi un interesse dell'autore in linea con le opzioni didattiche condivise da altri maestri della stagione po-

Prosdocimi, Alberico da Rosate; Billanovich, Epitafio, libri e amici di Alberico da Rosciate; Storti Storchi, Prassi dottrina ed esperienza legislativa; Quaglioni, Legislazione statutaria; Petolettr, «Ad utilitatem volentium studere in ipsa comedia»; CONETTI, La dottrina dell'impero; STORTI, Alberico da Rosciate, con ulteriore bibliografia; GiazZi, Alberico da Rosciate.

37 Alberico da Rosciate, Commentaria, al tit. Quae sit longa consuetudo (C. 8.52), proemio (f. 170rb): «Materia ista profunda est et utilis et per glossam et doctores sparsim tradita multis locis, quam infrascripto ordine colligere laboravi. Primo praemittendo utiles descriptiones, deinde contrarias quaestiones et distinctiones, et de ista materia tractatur per Azonem in Summa huius tituli et hic per Od(ofredum), Ni(cholaum) in Decisa et Cynum extra de verborum significatione $[\ldots] »$.

38 ID., Commentarium de Statutis, 1. III, q. 2, f. 55va: «Et sic concludendo tenet, quod si ex lege municipali sit facta publicatio, non extenditur ad bona alterius territorii, et tenet pro illa bona pertineant ad eum vel haeredes eius, ut in ipsa quaestione plenius tangitur, et incipit quaestio Quidam inculpatus de homicidio. Et de ista materia tangitur per Old(radum) [rectius: Odd(ofredum)] C. de haere. instit. 1. 1, et ibi per eum in Decisa Nic(olai) de Mata(rellis)». Già SolmI, Alberto da Gandino, p. 170, nota 2, riteneva attendibile la notizia riferita da Guglielmo da Pastrengo alla luce delle testimonianze concordi del Gandino.

$39 \mathrm{Si}$ tratta di cinque testamenti diluiti nell'arco di un quindicennio fra 1345 e 1360: CREMAschi, Contributo alla biografia di Alberico da Rosciate, p. 40 ss. e p. 43 per la citazione; per l'edizione dei cinque testamenti si veda p. 60 ss. «Purtroppo non si è ritrovato l'inventario definitivo e autografo, che, secondo un codicillo preposto al suo ultimo testamento, egli depose presso il Consorzio della Misericordia»: Billanovich, Cultura bergamasca del Trecento, p. 25. Sulla biblioteca di Alberico, che si può conoscere attraverso i suoi testamenti, si veda da ultimo Autographa, I.2., p. 86 ss. (voce curata da E. Giazzi). 
staccursiana quali, in primo luogo, Martino del Cassero, frequentato da Mattarelli nella propria città natale e attento alla formazione giuridica di alunni certamente meno 'pretenziosi' e facoltosi a confronto degli studenti patavini tramite la redazione di ben note raccolte di Notabilia sia a testi civilistici (Digesto e Istituzioni) che canonistici (Decreto e Decretali) $^{40}$.

Il particolare interesse a rimodulare secondo una cifra propria le Letture odofrediane, che affondano saldamente le radici nell'ambiente scolastico bolognese e nel personale impegno didattico del giurista contemporaneo ad Accursio, conduce a supporre una conoscenza diretta di Odofredo da parte del giovane Mattarelli offrendo, nel contempo, un indizio significativo per rischiararne la formazione universitaria, rimasta sino a ora nell'ombra causa l'assoluta mancanza di fonti specifiche ${ }^{41}$.

La frequentazione delle lezioni di Odofredo avrebbe quindi favorito una conoscenza approfondita delle Letture civilistiche elaborate dal proprio Maestro, il quale - tra l'altro - nel passato aveva pure avuto relazioni dirette, per quanto saltuarie, con la città e le istituzioni modenesi: nel dicembre 1249, assieme al giurista Pascipovero e a Salatiele, già suo allievo nello studio giuridico, Odofredo rappresenta il Comune bolognese alla stipulazione di un trattato di pace con Modena ${ }^{42}$ e nel luglio 1254 è inviato come ambasciatore direttamente a Modena allo scopo di comporre alcuni dissidi scaturiti all'interpretazione della pace siglata quasi cinque anni prima ${ }^{43}$.

40 In specie, su questi aspetti della produzione del giurista fanese, si veda SARTI, Martino da Fano e i suoi «Notabilia super Institutionibus»; EAD., Martino da Fano e $i$ suoi Notabilia super Institutionibus: una didattica per le università minori.

${ }^{41}$ A Manlio Bellomo si deve la cauta proposta di ritenere Mattarelli allievo di Nicola da Cremona sulla base di una additio apposta dal primo a margine di una quaestio civilistica del maestro cremonese trascritta in un noto codice vaticano: Bellomo, Giuristi cremonesi e scuole padovane, p. 140 s., e vedi anche sotto, cap. II, nota 91.

42 MSM V, n. 836, pp. 32-36, 1249 dicembre 15; Padovani, L'archivio di Odofredo, pp. 26, 47 s. Per il rapporto di discepolato di Salatiele nei confronti di Odofredo: Salatiele, Ars Notariae, I, p. XVII; Birocchi, Salatiele, p. 1769. Per Pascipovero, docente a Bologna e autore di una Concordia utriusque iuris redatta tra 1246 e 1253: BetTetini, Pascipovero.

43 Padovani, L'archivio di Odofredo, p. 29; Spagnesi, Denari Odofredo, p. 700; ID., Odofredo Denari, p. 1450. 
D'altro canto, nel legame dottrinario con Odofredo e con la sua opera di matrice scolastica si potrebbe anche ravvisare un orientamento del giurista modenese vòlto a privilegiare una linea meno rigidamente ancorata alla lezione culminata nella glossa accursiana; linea che trova in Ugolino Presbiteri e Iacopo Balduini, oltre che nell'opera odofrediana ${ }^{44}$, i propri modelli e che viene variamente sviluppata da altri autorevoli maestri attivi al di fuori dello Studium bolognese come - a titolo di esempio - Guido da Suzzara, il quale tiene cattedra a Modena per meno di due anni fra 1260 e 1262 prima di trasferirsi a Mantova e quindi a Venezia, Padova, Bologna e Napoli, ove fra il 1268 e il 1270 svolge il ruolo di consigliere di Carlo d'Angiò e di docente in quello Studio ${ }^{45}$.

\section{Lo Studio modenese nel Duecento e il primo magistero di Mat- tarelli}

A Modena lo Studio pare già stabilizzato intorno alla metà del secolo XII e nell'ultimo ventennio del secolo è accertata la presenza, sostanziata da un'operosa attività dottrinaria e anche dall'insegnamento giuridico, di Pillio da Medicina (1140 ca.-post 1207$)^{46}$, del

44 Allo sviluppo di una linea dottrinaria "alternativa" rispetto alle metodologie privilegiate dalla scienza dei glossatori e al primato della glossa accursiana, del resto non ancora consolidatosi pienamente entro l'orizzonte del secolo XIII, Manlio Bellomo ha dedicato - come ben noto - una prolungata serie di approfondimenti: si veda almeno Bellomo, Consulenze professionali e dottrine di professori, p. 200 ss.; ID., Intorno a Roffredo Beneventano: professore a Roma, p. 147 s.; ID., La scienza del diritto al tempo di Federico II, p. 176 ss.; ID., Sulle tracce d'uso dei «libri legales», p. 131 ss.; ID., L'Europa del diritto comune, p. 187 ss.; ID., Consulenze professionali e dottrine di professori, in part. pp. 19 s., 23 s.; ID., Tracce di lectura per viam quaestionum; ID., I fatti e il diritto, p. 385 ss. La tematica è ripresa anche da Speciale, La memoria del diritto comune, in part. p. 77 ss.

45 Per i dati biografici del maestro mantovano si fa riferimento a MAZzANTI, Guido da Suzzara, p. 421 s., e a Mazzanti-Murano, Guido da Suzzara, p. 19 ss. Per i caratteri originali della sua produzione dottrinaria, attestata tramite un numero cospicuo di manoscritti, si rinvia a MARTINo, Ricerche sull'opera di Guido da Suzza$r a$, e ID., Testimonianze sull'insegnamento del diritto a Napoli nei secoli XIII-XIV, oltre a Mazzanti-Murano, Guido da Suzzara, p. 22 ss.

46 Contese, Pillio da Medicina, p. 1588, con larga bibliografia finale a p. 1590. Sulla presenza e l'attività scientifico-didattica di Pillio a Modena si vedano, 
quale sono colleghi Guido da Pavia e Guizzardo da Colorno, che ricoprono a più riprese incarichi comunali fra il 1181 e il $1219^{47}$. Nella prima metà del Duecento tengono scuola a Modena Alberto da Pavia ( $†$ post 1243), che nel complesso e in modo intermittente vi insegna per quasi trent'anni ${ }^{48}$, Uberto di Bonaccorso ( $\dagger$ post 1240 ), modenese di origine e allievo di Azzone ${ }^{49}$, e Uberto da Bobbio (1185 ca.-1245), che dal 1237 rientra definitivamente a Parma e dall'anno precedente risulta già sostituito, da allora e per un ventennio, da Giovanni da Nonantola ${ }^{50}$.

Nell'arco di un triennio, probabilmente tra il 1242-43 e il 124445, insegna a Modena il cremonese Omobono Morisio ( $† 1262$ ), già allievo in questa stessa città di Alberto da Pavia e Uberto da Bobbio $^{51}$, e nel periodo 1256-59 Martino da Fano (1190 ca.-post

in particolare, Vicini, Profilo storico, p. 5 ss.; Mor-Di Pietro, Storia dell'Università di Modena, I, p. 4 ss.; FrIed, Die Entstehung, p. 188 s.; SANTINI, Università e società nel XII secolo, p. 161 ss.; Autographa, I.1., pp. 7-10 (voce curata da E. Tavilla).

47 Vicini, Profilo storico, p. 4, secondo il quale le testimonianze documentarie relative al primo, puntualmente richiamate in nota, si datano fra 1180 e 1205 , mentre quelle relative al secondo fra il 1188 e il 1211; Mor-Di Pietro, Storia dell'Università di Modena, I, p. 7; SANTINI, Università e società nel XII secolo, p. 236 s.; RöLKer, Nobiltà e Comune a Modena, pp. 187 s., 201 ss.

48 Vicini, Profilo storico, p. 8; Sorrenti, Tra «lecturae»e «quaestiones», p. 115 e nota 60; SANTINI, Università e società a Modena, p. 359 ss.; Bordini, Per un profilo di Uberto da Bobbio, p. 71 s., nota 23.

49 VicinI, Profilo storico, p. 11 s.: è presente con certezza a Modena fra 1231 e 1236; SANTINI, Giuristi settentrionali; SofFIETTI, Uberto di Bonaccorso. Uberto, oltre a far parte della schiera di giuristi di cui si circondò Federico II al tempo dell'istituzione dello Studio napoletano, dallo stesso imperatore è inviato a Vercelli a tenere cattedra di leggi in una data collocabile tra 1238 e 1240.

50 VicinI, Profilo storico, p. 12, ove si colloca l'attività di insegnamento di Uberto a Modena negli anni 1214 e 1227 . Anche sugli altri giuristi citati in precedenza e la loro attività di docenza a Modena si veda TiRaboschi, Biblioteca modenese, I, p. 49 ss.; Mor-Di PIETro, Storia dell'Università di Modena, I, p. 11 e II, p. 329; Fried, Die Entstehung, pp. 187, 193 ss., 197 ss., 199, 204 ss., 210, 216 ss., 223 ss., 239; RöLKer, Nobiltà e Comune a Modena, pp. 188, 201 s., 235 ss., 252; BordinI, Per un profilo di Uberto da Bobbio, p. 87 ss. Un'approfondita e recente analisi del noto libello di Uberto da Bobbio dedicato a fornire istruzioni pratiche e deontologiche a quanti esercitano la professione forense, con frequenti legami allargati ad altri maestri duecenteschi variamente collegati a Modena quali Alberto da Pavia, Uberto di Bonaccorso e Guglielmo Durante, si deve a SARTI, Il Liber cautele et doctrine, con un'ulteriore sintesi biografica in ID., Uberto da Bobbio.

51 GualazzinI, Contributi alla storia della scuola giuridica cremonese, p. 110, ove si trascrive una parte del testamento del maestro, già edito in Astegiano, Co- 
$1272)^{52}$, il quale, chiamato a ricoprire a Genova l'ufficio di podestà, dal 1260 è sostituito dal celebre Guido da Suzzara, che riceve dal Comune la cifra esorbitante di 2.250 lire, la maggior parte delle quali (1.250) avrebbero dovuto essere investite in beni immobili nella città e nel distretto, dietro promessa di porre in perpetuo la propria residenza in Modena, offrire consulenza gratuita alle autorità comunali, leggere nello Studio senza ricevere alcun salario dagli studenti e, infine, non prestare patrocinio ad alcuno eccetto gli studenti forestieri ${ }^{53}$.

Se l'attività modenese di Guido resiste unicamente due anni, interrotta dal suo repentino trasferimento a Mantova e dall'apertura di una controversia con il Comune risolta soltanto vent'anni più tardi ${ }^{54}$, nel settimo decennio del Duecento lo Studio modenese vanta la presenza di Guglielmo Durante ${ }^{55}$ e poco dopo dello stesso Mattarelli, il quale dal

dex diplomaticus Cremonae, I, n. 722, p. 305, 1259 luglio 15, con codicilli aggiunti in data 1261 agosto 31; in esso il maestro cremonese precisa di avere insegnato a Modena per un periodo complessivo di tre anni, due cum salario et per unum annum sine (p. 307); Loschiavo, Morisio Omobono, p. 864; ID., Omobono Morisio, p. 1456; Bordini, Per un profilo di Uberto da Bobbio, p. 96 s. Posticipano invece tra 1255 e 1259 il magistero modenese di Omobono Mor-Di Pietro, Storia dell'Università di Modena, I, p. 12.

52 Tavilla, Homo alterius, p. 201; Vicini, Profilo storico, p. 13 s.; LiotTa, Del Cassero Martino, p. 443; Semeraro, Martino del Cassero, p. 1291. Altri maestri sono attivi a Modena negli anni centrali del Duecento come Giovanni da Nonantola e il parmense Alberto Galeotti: VicinI, Profilo storico, p. 12; SANTINI, Università e società nel XII secolo, p. 152 s.; IsotTon, Galeotti Alberto, p. 929.

53 Il documento, datato 6 aprile 1260, è trascritto in Torelli-Vicini, Documenti su Guido da Suzzara, III, pp. 323-325, e in RPCM, II, n. 330, pp. 207-211. Al perfezionamento del contratto tra Guido da Suzzara e le autorità comunali modenesi fanno riferimento anche altri atti successivi datati fra I maggio e 15 settembre 1260: Torelli-Vicini, Documenti su Guido da Suzzara, IV-IX, pp. 325-33 (= RPCM, II, nn. 331-335, pp. 211-222). Si veda Mor-Di PIETRo, Storia dell'Università di Modena, I, p. 9 ss. anche per i riferimenti precedenti. Aggiornate sintesi biografiche relative a Guido da Suzzara si devono a MAzzAnti, Guido da Suzzara, a p. 421 s. per i suoi rapporti con il Comune modenese, e a BenATti, Guido da Suzzara.

54 Tra i documenti che attestano la risoluzione di tale controversia si ricorda anche il pagamento di 40 lire modenesi da parte di Gerardo da Costrignano, sindaco del Comune di Modena, al legum doctor Guido da Suzzara assolvendolo da ogni obbligazione verso il Comune stesso; atto stipulato nel Palazzo nuovo del Comune di Modena in presenza, come testimoni, del legum doctor Nicholaus Mattarelli, di Tommaso da Spezzano e Corradino de' Migliarini: ASMo, Memoriale antico, a. 1280, n. 1414, I agosto 1280 .

55 Sul maestro di origine transalpina si rinvia all'ampia bibliografia citata in Colli, Lo Speculum iudiciale di Guillaume Durand, in specie p. 517 ss., assieme a 
1270 - come sopra ricordato - nella documentazione scritta viene regolarmente qualificato mediante i titoli, equivalenti, di doctor legum o legum professor, in cui si riflette con evidenza il magistero giuridico da lui condotto da tale altezza cronologica ${ }^{56} \mathrm{e}$ continuato in patria - stando all'opinione di Carlo Guido Mor - almeno per un decennio ${ }^{57}$.

Un riscontro diretto di tale attività didattica emerge dieci anni dopo, quando il 6 agosto 1280 Gerardo da Costrignano, sindaco del Comune di Modena, si impegna a corrispondere a Nicholao de Matarello doctor legum la somma di 100 lire bolognesi o modenesi, metà alla festa di Ognissanti e metà a Natale, per remunerare l'insegnamento che egli dovrà tenere a partire dalla festa di san Michele (29 settembre) dello stesso anno e per il successivo leggendo librum exordinarium bona fide ad utilitatem scolarium comunis Mutine ${ }^{58}$.

Oltre a individuare nel librum exordinarium una delle parti dei libri legales differenti dal Codice e dal Digesto vecchio, ritenuti per consolidata tradizione didattica basi irrinunciabili delle letture ordinarie $^{59}$, è da notare come il compenso offerto a Mattarelli dal Comune, che fin dal XII secolo sostiene e finanzia in modo diretto lo Studio pubblico modenese ${ }^{60}$, sia in linea con i valori riscontrabili in diversi casi di incarichi conferiti a prestigiosi doctores legum ancora lungo il Trecento, benché il salario potesse incrementarsi in ragione della notorietà del maestro o della generosità del committente motivata dal desiderio di accaparrarsi comunque un docente di fama.

Il primo giugno 1279 Guido da Suzzara, reduce dall'insegna-

Roumy, Durand (Durant, Durandi) Guillaume: Durante insegna il Decreto a Modena probabilmente tra 1260 e 1264. Secondo Murano, Guillaume Durand, p. 49, studia diritto a Bologna tra 1250 e 1260 e si addottora in diritto canonico nel 1263.

56 Opzione interpretativa che già SAVIGNY, Storia del diritto romano, II, p. 453, risolveva con sicurezza in favore della rispettiva equivalenza e proprio in attinenza al caso di Niccolò Mattarelli.

57 Mor-Di Pietro, Storia dell'Università di Modena, I, p. 12, secondo cui Mattarelli succede a Guglielmo Durante, «certo dal 1272», fra gli «insegnanti di grido» attivi a Modena.

58 ASMo, Memoriale antico, a. 1280, n. 97. L'atto, ricordato in VICINI, Di Nicolò Mattarelli, p. 14 e in Murano, Paolo di Jacopino Avvocati, p. 26, è trascritto in Bedoni, Nicolò Mattarelli, p. 87 s.

59 Bellomo, Saggio sull'Università, p. 153.

60 Si ricordi lo stipendio offerto dal Comune di Modena a Pillio da Medicina intorno al 1180, che ammontava, per testimonianza dello stesso maestro, a 100 marche d'argento: ibidem, p. 144. 
mento a Reggio protrattosi dal 1270 al 1278 , stipula un contratto con i rettori degli studenti di Bologna per insegnare nella stessa città il Digesto nuovo dietro un compenso notevole di 300 lire bologne$\mathrm{si}^{61}$. L'anno successivo il canonista Garsia, dopo avere tenuto come supplente la scuola di Egidio dei Foscherari, riceve 150 lire dal Comune di Bologna per insegnare in modo autonomo il Decreto per un intero anno scolastico. Uguale stipendio viene offerto dalle stesse autorità comunali nel 1289 al canonista Altigrado da Lendinara, al quale viene affiancato, per il diritto civile, Dino Rossoni del Mugello, incaricato di tenere una cattedra straordinaria dell'Inforziato e del Digesto nuovo con il compenso di 100 lire $^{62}$.

Una somma un po' più alta, pari a 130 lire imperiali, viene offerta allo stesso Mattarelli nel giugno 1292 non dal Comune di Cremona, bensì dalla Universitas scolarium Cremone in iure civili per leggere i libri del Codice, dell'Authenticum e i Tres Libri con apparato accursiano durante il successivo anno scolastico a partire dalla data tradizionale del 29 settembre ${ }^{63}$.

61 Ibidem, p. 151; Mazzanti, Guido da Suzzara, p. 423.

62 Coppr, Le università italiane nel medio evo, p. 228; Bellomo, Saggio sull'Università, p. 151 s.; Padovani, Dino Rossoni, p. 769; Murano, Dino del Mugello, p. 54. Sul tema della remunerazione dei maestri tramite collette studentesche oppure tramite stipendi corrisposti da Comuni o autorità pubbliche si veda anche PosT, $M a$ ster's salaries, in particolare p. 193 ss. per alcuni casi relativi a città comunali italiane, privo tuttavia di esempi concernenti l'importo specifico dei salari pagati ai docenti.

63 CAPpelli, Dizionario di abbreviature latine, Tavola IV, pp. LXII-LXIV, con trascrizione e riproduzione fotografica del documento datato 23 giugno 1292, già trascritto in Romano, Un documento cremonese, p. 145 s., ma con data errata 8 giugno. L'atto è ricordato in Murano, Paolo di Jacopino Avvocati, p. 26. Nel documento si specifica che il maestro dovrà attendere all'insegnamento di persona, senza farsi sostituire da altri, svolgendolo in modo regolare e continuativo. Pochi giorni dopo, sabato 28 giugno, nel Consiglio generale del Comune di Cremona riunito super palacio veteri comunis Cremone e in presenza del Capitano del Popolo Barufaldino de Lavello si incarica magistrum Iohannem bidellum, quale sindaco e procuratore del comune, di recarsi a Modena ad domum nobilis viri domini Nicolay Mattarelli doctori et domino legum et honorabili civi Mutine per comunicargli l'elezione fatta dagli scolares legum Cremone e promettergli tuttavia il solo salario previsto dallo statuto, ossia 50 lire imperiali ad monetam Cremone da corrispondersi per metà in occasione della festa di tutti i Santi e per metà a Pasqua: AstegiAno, Codex diplomaticus Cremonae, I, n. 1124, p. 385. Anche Gualazzini, Nuovi contributi per la storia dello «Studium» di Cremona, p. 101, ricorda la chiamata di Mattarelli a Cremona nel 1292 e considera probabile la sua effettiva docenza presso lo Studium cittadino; vedi anche p. 110 s. per il funzionamento di quest'ultimo 
Nel 1310 a Bologna Osberto da Cremona è incaricato delle lettura straordinaria dell'Infortiatum per il triennio seguente con il salario di 100 lire bolognesi (verosimilmente annue), mentre un salario di 150 lire è offerto al maestro, ugualmente cremonese, Egidiolo Madalberti per la lettura del Decretum ${ }^{64}$. Analogo salario di 100 lire viene offerto a Osberto nell'ottobre 1313 dal Comune di Perugia per tenere la cattedra ordinaria di diritto civile nei tre anni successivi ${ }^{65}$, al termine dei quali, rientrato a Bologna, tiene dal 1316 al 1318 la lettura straordinaria del Digesto Nuovo e dell'Inforziato ancora dietro compenso di 100 lire bolognesi ${ }^{66}$, che per il 'mercato' della cultura universitaria del tempo si può valutare come un salario comunque non elevato ${ }^{67}$. Sempre a Bologna nel 1324 Ranieri Arsendi, nato a Forlì verso fine Duecento e giovane docente nella stessa città dal 1319-20, riceve uno stipendio di 100 lire per leggere il Digesto Nuovo ${ }^{68}$. Analogo compenso annuo, da corrispondere in tre rate, è fissato in uno statuto modenese del 1328 per remunerare unum doctorem sive lectorem terrigenum conventatum in artibus ad legendum medicinam, allo scopo precipuo di fornire un insegnamento medico agli studenti modenesi impossibilitati a recarsi a Bologna per addottorarsi in medicina a causa delle spese ingenti69.

$\mathrm{Su}$ valori notevolmente più elevati si colloca invece, nei primi decenni del Trecento, il compenso offerto al giovane Bartolo, ad-

in forma indipendente dalla sola iniziativa delle autorità comunali. Esattamente 30 anni prima, nel 1262, era morto a Cremona Omobono Morisio, rientrato in patria da poco più di un decennio per dedicarsi all'attività di insegnante, di consulente e di giudice, ed era stato tumulato nella chiesa cittadina di S. Guglielmo: ID., Contributi alla storia della scuola giuridica cremonese, p. 84; Loschiavo, Morisio Omobono, p. 864; ID., Omobono Morisio, p. 1456.

64 Ghirardacci, Della Historia di Bologna, libro XVII, p. 549; Semeraro, Osberto da Cremona, p. 17.

65 Semeraro, Osberto da Cremona, p. 19. Successivamente all'ampia biografia umana e dottrinaria del giurista scaturita dalle ricerche di Martino Semeraro, un ulteriore contributo per ipotizzare un soggiorno di Osberto anche a Vercelli, benché in un periodo non facilmente determinabile, o comunque un legame con la città piemontese sede di uno Studium attivo in modo discontinuo tra Due e Trecento viene in anni recenti da MAFFEI, Osberto da Cremona.

66 Ghirardacci, Della Historia di Bologna, libro XVIII, p. 585; Semeraro, Osberto da Cremona, p. 20.

${ }_{67}$ Coppi, Le università italiane nel medio evo, p. 227 s.; Semeraro, Osberto da Cremona, p. 20, nota 67.

68 AbBondanza, Arsendi Raniero, p. 333.

69 Bedoni, La medicina a Modena, p. 76. 
dottoratosi a Bologna nel settembre-novembre $1334^{70}$, al quale il Comune di Pisa nel 1339, per il suo primo incarico di insegnamento, offre 150 fiorini d'oro ${ }^{71}$. Pochi anni prima, nell'autunno 1325 , Osberto, tornano di recente a Perugia e divenuto docente di fama, viene confermato un altro anno presso lo Studio cittadino con un salario di 300 fiorini d'oro, corrispondente circa a 600 lire bolognesi ${ }^{72}$.

A cavallo tra gli anni Settanta e Ottanta del secolo XIII Niccolò Mattarelli appare quindi agli inizi dell'attività di docente e lo stipendio offerto dal Comune della sua città di origine, forse perché già dissanguato anni prima dall'elevatissimo compenso offerto a Guido da Suzzara in cambio di prestazioni rapidamente disattese dall'irrequieto maestro, si allinea su valori non particolarmente elevati ${ }^{73}$; valori che tuttavia, integrati da collette riscosse con forte probabilità dagli studenti, dai proventi derivati dagli arbitrati e da una importante attività di compravendita e di locazione immobiliare, gli permettono di acquisire nel tempo una significativa disponibilità economica e di finanziare la scrittura di codici giuridici, imprescindibili per la sua professione, e non soltanto.

\section{Denaro, case, terre}

I cespiti assicurati dall'insegnamento vengono integrati da un'ulteriore fonte di reddito che emerge dalle fonti in modo indiretto e che si deve ritenere conseguente allo svolgimento di una diversa attività sempre connessa alla sapienza giuridica acquisita dal maestro modenese. Mattarelli, infatti, e dopo di lui anche il figlio Francesco dal $1293^{74}$, è

70 Il contesto nel quale si colloca la laurea bolognese di Bartolo, segnato da violenti contrasti conseguenti alla cacciata del legato papale Bertrando del Poggetto, è approfondito da Padovani, Un sermo di Cino da Pistoia, p. 28 ss., che comprova la presenza di Cino nella città emiliana tra l'ottobre del 1334 e i primi mesi dell'anno successivo.

71 Calasso, Bartolo da Sassoferrato, p. $641 \mathrm{~s}$.

72 Semeraro, Osberto da Cremona, p. 26 e nota 99.

73 Sui salari percepiti dai maestri nel tardo Duecento, che risultano sensibilmente accresciuti nel corso del secolo successivo, si vedano i numerosi esempi riportati in SAVIGNY, Storia del diritto romano, I, p. 585 ss.

${ }_{74}$ ASMo, Memoriale antico, a. 1293, n. 826 (9 febbraio 1293): Iacopinus de Gorgadelis con la figlia Bartolomea, da una parte, e Zanne del fu Ugolino, dall'altra, affidano a Francesco di Niccolò Mattarelli il compito di giudicare come arbitro una 
ricercato nell'ambiente cittadino per lo svolgimento di arbitrati destinati a comporre controversie che interessano tanto soggetti di condizione laica quanto religiosa e pure varie istituzioni ecclesiastiche radicate in città e nel contado. Si tratta di vertenze per gran parte di natura fondiaria e patrimoniale che, a partire dal 1279 e sino al 1283, accanto a singoli proprietari privati ${ }^{75}$ vedono coinvolti il Capitolo della pieve di Massa $^{76}$, la domus dei Templari di Modena ${ }^{77}$ e il monastero benedettino di Nonantola ${ }^{78}$.

Le sintetiche registrazioni leggibili nei Memoriali notarili modenesi non consentono di evincere dati più ricchi e precisi né sul

questione dotale sorta tra loro. Ibidem, n. 827 (pari data): il doctor legum Francesco di Niccolò Mattarelli pronuncia un lodo (non meglio specificato) sulla questione dotale dietro richiesta delle parti.

75 ASMo, Memoriale antico, a. 1279, n. 3078 (14 dicembre 1279): il dominus Nicholaus Matareli e il dominus Coradinus de Miniarinis (Corradino de' Migliarini) pronunciano un lodo in base al quale Tobia Rangoni e Gerardino del fu Iacopino Rangoni devono corrispondere l'ingente somma di 200 lire modenesi a Castellano, servitore del fu Iacopino Rangoni, secondo quanto specificato nell'atto redatto dal notaio Mutinensis fratris Valentini. ASMo, Memoriale antico, a. 1280, n. 1848 (27 novembre 1280): Giuliano del fu frater Scaminax, rappresentato da Antonio de Pizollbechariis da una parte, e Simone de Carnelvariis, dall'altra, eleggono Niccolò Mattarelli quale arbitro nella questione sorta tra loro in merito alla compravendita di due terreni. ASMo, Memoriale antico, a. 1283, n. 2559 (26 giugno 1283): Niccolò Mattarelli, eletto dai fratelli Iacopo e Giovanni figli del fu Alberto Riticheldi e da Giovanni Restani, pronuncia sentenza (non meglio specificata) in merito a una questione inerente una terra nel distretto di Vignola. ASMo, Memoriale antico, a. 1283, n. 1 (1 luglio 1283): Niccolò Mattarelli, scelto quale arbitro sempre dai fratelli Iacopo e Giovanni, figli del fu Alberto di Simone Riticheldi, da una parte e da Giovanni Restani dall'altra, dispone in merito alla lite vertente tra essi che Giovanni Restani restituisca ai due fratelli due moggi di frumento e altri beni mobili.

76 ASMo, Memoriale antico, a. 1279, n. 3175 (2 gennaio 1279): il sindaco del Capitolo della pieve di Massa, Ardizio da Lovoleto, a nome della medesima pieve e del Capitolo, da una parte, e Zanino, Gerardino e Stefanino da Bagnolo, dall'altra, nominano il dominus Nicolaum de Matarello e Graziadio Andriani arbitri nella questione vertente tra di loro; tra i testimoni Gian Novello, fratello di Niccolò. Ibidem, n. 3176 (2 gennaio 1279): le parti insistono presso Mattarelli affinché definisca la questione ed egli sentenzia che i suddetti da Bagnolo debbano corrispondere alla pieve di Massa la somma di 9 lire modenesi in una parte.

77 Vedi sotto nota 98.

78 ASMo, Memoriale antico, a. 1280, n. 1665 (29 ottobre 1280): in presenza di Niccolò Mattarelli e di Corradino de' Migliarini viene composta la lite in merito a terre non meglio specificate fra il monastero di Nonantola, rappresentato dal sindaco donnus Petrus, e Maria del fu Iacopo del fu Guido Passaponti, la quale è tenuta a corrispondere al monastero la somma di 180 lire modenesi. 
compenso pattuito dal noto giurista, assieme pure ad altri esperti che in alcuni casi lo affiancano nello svolgimento di taluni arbitrati, né sulla qualità giuridica della funzione arbitrale svolta, se limitata al ruolo di arbitrator allo scopo di definire la vertenza in base a soli principi di equità (ex bono et aequo), senza vincoli in procedendo e in forma transattiva, secondo una soluzione compromissoria più vicina al modello dell'arbitrato delineato dalle fonti romane, oppure formalizzata in quello di arbiter, chiamato a risolvere una controversia civile secondo norme positive e secundum iudiciorum ordinem formulando una sentenza che fosse pertanto appellabile dinanzi al giudice ordinario ${ }^{79}$. Pur nella laconicità delle testimonianze disponibili, il riferimento esplicito alla pronuncia di una sentenza da parte del solo giurista, nei due casi in cui egli risulta unico titolare di tale funzione, suggerisce che la configurazione dell'operato di Mattarelli fosse piuttosto aderente a quest'ultimo profilo, lasciando comunque aperta la possibilità, in tutti gli altri, che egli sia stato chiamato a emanare anche lodi, grazie al suo prestigio professionale oltre che alla sua indubbia competenza legale, sulla base piuttosto di amicabiles compositiones richieste e accettate dalle parti.

Accanto ai profitti assicurati dall'attività di insegnamento e dallo svolgimento di arbitrati su incarico di numerosi soggetti di condizione laica quanto ecclesiastica, Mattarelli deriva una parte significativa della propria ricchezza dalla gestione, tramite contratti di

79 Le linee essenziali dell'istituto, così come evolutosi nella dottrina e nella prassi di età intermedia, sono delineate in PIANO MORTARI, Arbitrato (diritto intermedio). Indagini più recenti sull'argomento e sul contributo offerto dalla riflessione civilistica e canonistica, dal tardo XII secolo, alla puntuale definizione teorica della materia si devono a Fowler, Forms of Arbitration; MARTONE, Arbiter-arbitrator, in specie p. 73 ss.; Meccarelli, Arbitrium, p. 54 ss.; Tenella Sillani, L'arbitrato di equità, p. 70 ss. Con la premessa di un'ampia rassegna di ulteriore bibliografia e fonti dottrinarie, il complessivo sviluppo storico dell'istituto è tracciato da Piergiovanni, Profili storici. Ai fondamenti romanistici è dedicato in particolare BuIGUES, La solucion amistosa de los conflictos. Un recente esame delle due figure arbitrali, in specie attraverso il riscontro dei formulari notarili duecenteschi sino alla grande sistemazione della materia operata da Roladino, è condotto da STORTI STORсні, Compromesso e arbitrato, p. 333 ss. L'approfondimento di un caso specifico, calato nell'esperienza della società e delle istituzioni veneziane tra basso medioevo e prima età moderna sulla base di una folta documentazione inedita costituita da fonti consiliari e notarili, si deve a Marrella-Mozzato, Alle origini dell'arbitrato commerciale internazionale, in part. p. 38 ss. per il richiamo alla dottrina di diritto comune. 
compravendita e di affitto, di un patrimonio fondiario dislocato in città e in varie località del contado, che in parte viene amministrato assieme ai due fratelli; per altra parte più cospicua esso è frutto di operazioni economiche condotte in forma indipendente.

Derivano verosimilmente dall'eredità lasciata dal padre Giovannibuono, e vengono pertanto amministrati in modo indiviso, $i$ terreni che a partire dal 1272 il legum doctor Niccolò vende oppure concede in affitto unitamente ai fratelli Gian Novello e Alessandro ${ }^{80}$, ubicati in luoghi diversi nella fascia di pianura non lontano da Modena, soprattutto nella zona a settentrione della città nei pressi di Panzano $^{81}$ e Villavara ${ }^{82}$.

Un ultimo acquisto effettuato assieme ai due fratelli sullo scorcio del 1277 ha per oggetto un terreno ubicato nel distretto di Gorzano, una decina di chilometri a sud di Modena ${ }^{83}$, mentre sempre

80 ASMo, Memoriale antico, a. 1272/1, n. 2148 (20 febbraio 1272): dominus Nycholaus legum doctor, magister Iohannes Novellus, Allexander fratres qd. Domini Iohannisboni Mattarelli vendono a Bernardo filio olim domini Petri de Suzo da Vignola un terreno arativo dell'estensione di 3 biolche ubicato super Formigine vetere per la somma di 27 lire modenesi. Sul margine sinistro della registrazione sono evidenziati in colonna i nomi degli attori della vendita: dominus Nycholaus legum doctor, magister Iohannes Novellus, Allexander.

81 ASMo, Memoriale antico, a. 1273, n. 2873 (30 marzo 1273): i fratelli Gian Novello, Niccolò e Alessandro figli del fu Giovannibuono de Matarello vendono in Modena a Guglielmo del fu Iacopino Tedelendi e ai suoi fratelli Gianbuono e Sigizo un terreno di oltre 15 biolche ubicato nella curia di Panzano al prezzo di 148 lire modenesi. Panzano è identificabile con una località situata a oriente di Modena vicino al corso del fiume Panaro: DTS II, p. 179 s.

82 ASMo, Memoriale antico, a. 1278, n. 2062 (25 novembre 1278): Niccolò Mattarelli e Gian Novello, agendo anche per il fratello Alessandro, concedono in locazione ad laborandum ai tezolani Carnevale e Albertina tutte le terre che essi possiedono nella villa di Villavara. Sul significato del termine tezolani, quali coltivatori di condizione non libera, presenti anche all'interno di proprietà ecclesiastiche, che potevano detenere in proprietà non più di quattro biolche di terra, cfr. RÖLKER, Nobiltà e Comune a Modena, p. 234. Villavara è attualmente una località in comune di Bomporto, a nord-est di Modena e non lontano da Nonantola.

83 ASMo, Memoriale Antico, a. 1277, n. 4955 (22 dicembre 1277): Albertino del fu Martino da Sala vende al dominus magister Gian Novello e ad Alessandro, figli del fu Giovannibuno de Matarello, i quali acquistano per sé e per il fratello Niccolò, un terreno posto nel distretto di Gorzano al prezzo di 10 lire imperiali. Anche in corrispondenza dell'anno precedente sono registrati nei Memoriali notarili i sunti di altri tre atti relativi a transazioni immobiliari e mutui che vedono coinvolti solidalmente i fratelli Niccolò, Alessandro e Gian Novello: ASMo, Memoriale antico, a. 1276, n. 949 (5 febbraio 1276); n. 1260 (4 maggio 1276); n. 4103 (31 ottobre 1276). 
presso Villavara ancora nel 1289 Niccolò Mattarelli, tramite procura data al figlio Francesco, acquista alcuni appezzamenti di terre gravati - come pare - da un vincolo livellario in favore di un soggetto che non viene specificato nella registrazione compendiata dell'atto notarile a noi pervenuta ${ }^{84}$; e ancora due anni dopo con analoghe modalità Mattarelli dispone una permuta di terre situate nel medesimo $\operatorname{luog} 0^{85}$.

Non lontano da Villavara e dal contiguo territorio di Nonantola, sede dell'ancor prestigioso monastero benedettino fondato alla metà del secolo VIII, nel marzo 1283 Mattarelli investe la non piccola somma di 100 lire imperiali, pari a un anno di salario di insegnamento, nell'acquisto di un terreno fornito di abitazione posto nella villa di Navicello, poco a ovest del corso del fiume Scoltenna-Panaro ed entro il raggio di una decina di chilometri dalla città ${ }^{86}$.

Un altro cospicuo investimento fondiario è realizzato da Mattarelli nel 1280, all'epoca dell'acquisto di una residenza urbana nella contrada del Castellaro ${ }^{87}$, comprando per 139 lire imperiali un complesso di 10 appezzamenti di terreno situati nella campagna a ovest del centro cittadino, in direzione della località di Magreta di cui è originario il venditore ${ }^{88}$.

84 ASMo, Memoriale antico, a. 1288-89/1, n. 952 (20 aprile 1289): Francesco figlio Nicholay Matharelli, quale procuratore del padre, acquista da Gerardo de $\mathrm{Au}$ gustis e dai suoi figli Martino e Gianbuono iure libelli tre appezzamenti di terreno situati nel distretto di Villavara e nella curia di Nonantola per la somma di 22 lire, 11 soldi e 1 denaro. Considerata l'ubicazione dei beni, si può supporre che il proprietario eminente degli stessi sia il vicino monastero di S. Silvestro di Nonantola.

85 ASMo, Memoriale antico, a. 1291, n. 4136 (1 dicembre 1291): Francesco Mattarelli, come procuratore del padre, permuta con Giliolo de' Canonici alcune terre ubicate nel distretto di Villavara.

86 ASMo, Memoriale antico, a. 1283, n. 1978 (23 marzo 1283): il legum doctor Niccolò Mattarelli compra da Rainaldo Borghesani per la somma di 100 lire imperiali un terreno con casa nella villa di Navicello, esteso per 90 biolche e situato tra il corso del Panaro e la fossa Monda.

87 Vedi sotto nota 95.

88 ASMo, Memoriale antico, a. 1280, n. 5977 (23 aprile 1280): Niccolò Mattarelli compra da Bernardino del fu Arduino de Macreto, attraverso il procuratore Ugolino de' Crespi, 10 appezzamenti di terreno ubicati nella curia di S. Salvatore al prezzo complessivo di 139 lire imperiali. Murano, Copisti a Bologna, p. 32, nota 106 , ricorda questo atto come prova delle ampie disponibilità economiche del giurista. S. Salvatore è identificabile con uno dei due nuclei in cui si articolava la località di Freto, tuttora esistente poco a ovest di Modena: DTS I, p. $321 \mathrm{s.}$ 
Altri acquisti di terre rustiche e di immobili urbani sono effettuati tre anni dopo dal giurista modenese, il quale diversifica il proprio capitale anche nell'acquisto di animali, nel caso di specie ovini, da cui ricavare lana e formaggi per approvvigionamento famigliare, che conferisce a un allevatore tramite il collaudato contratto di soccida $^{89}$. Nel caso del contratto stipulato nel 1283 Mattarelli probabilmente investe una parte dei guadagni che gli ha fruttato l'incarico di consultore a Foligno, svolto nel corso dell'anno precedente ${ }^{90}$, acquistando da Pietro del fu Antonio da Roteglia le porzioni di sua pertinenza di diversi appezzamenti di terra con case sopra edificate nel territorio della villa di Corlo superiore, nella fascia di alta e fertile pianura a sud di Modena. Dallo stesso Pietro, Mattarelli compra anche una porzione di un terreno situato in città in contrata de Muro ${ }^{91}$, una via compresa nel quartiere di porta Baggiovara, corrispondente al settore sud-occidentale della città, ove già alcuni anni prima il giurista aveva acquistato altri immobili nell'ambito di una sequenza che lo porta ad accumulare un significativo patrimonio dislocato in zone ben precise dell'area urbana.

Nel settore sud-orientale di Modena occupato dal quartiere di porta San Pietro si trova un terreno con casa, posto in contrata de Malclavellis, che Niccolò Mattarelli vende a Francesco del fu Gerar-

89 ASMo, Memoriale antico, a. 1285, n. 533 (21 febbraio 1285): Tomasino Ungarini promette a Gian Novello Mattarelli, in qualità di procuratore del fratello Niccolò, di accettare 67 pecore in soccida dalla festa di tutti i Santi per 3 anni. Sulla soccida medievale, un contratto di natura associativa volto a definire una collaborazione economica tra chi è proprietario del bestiame e chi si incarica del suo allevamento, disponiamo di alcuni studi dedicati al territorio bolognese e a quello modenese contermine: Francia, Il contratto di soccida nel Bolognese; ANDrEOLLI, La soccida nel territorio mirandolese; COSER-GIANSANTE, Le locazioni di bestiame nel bolognese fra XIII e XIV secolo; ID., Libro di conti della famiglia Guastavillani, p. 50 ss.

90 Per il quale si veda di seguito, paragrafo 7.

91 ASMo, Memoriale antico, a. 1283, n. 4583 (23 gennaio 1283): il legum doctor Niccolò Mattarelli acquista per la somma complessiva di 50 lire modenesi da Pietro del fu Antonio da Roteglia le porzioni di diversi appezzamenti di terreno con casa posti nella villa di Corlo superiore assieme alla porzione di un terreno situato in Modena in contrata de Muro confinante a mane con la detta strada et a sero circa, ovvero con la fossa circondaria della città nel settore di sud-ovest, all'interno del quartiere di porta Baggiovara. Fra i testimoni è presente Gian Novello, fratello di Niccolò. 
do Guidoni nell'ottobre $1273^{92}$; un altro terreno edificato posto in contrada de Ferariis, all'interno dello stesso quartiere, viene ceduto due anni dopo a Niccolò e ai suoi fratelli direttamente dalla moglie del doctor legum, Albertina del fu Albertino da Montevallaro, quale saldo di un debito di 40 lire modenesi ancora aperto nei loro confronti ${ }^{93}$. Sempre nel 1275 Niccolò acquista dai frati Eremitani altri due appezzamenti in parte edificati nel vicino quartiere di porta Baggiovara entro la cinquantina di S. Barnaba, una delle minori circoscrizioni in cui risulta ripartito lo spazio urbano e quello immediatamente esterno alla città secondo una nuova suddivisione di carattere topografico-amministrativo, che si delinea a partire dalla metà del XIII secolo, funzionale allo svolgimento di compiti legati alla sicurezza pubblica e alla riscossione fiscale ${ }^{94}$.

In una posizione ancora più vicina al cuore politico e religioso della città rappresentato dalla piazza sulla quale si affacciano i pa-

92 ASMo, Memoriale antico, a. 1273, n. 4695 (8 ottobre 1273): Nicholaus Mattarelli vende in Modena a Francesco del fu Gerardo Guidoni un terreno con casa ubicato in porta S. Petri in contrata de Malclavellis al prezzo di 63 lire modenesi.

93 ASMo, Memoriale antico, a. 1275, n. 2667 (11 settembre 1275): Albertina del fu Albertino da Montevallaro, moglie di Niccolò, cede al marito e ai cognati Giovanni e Sandro (così indicati, corrispondenti a Gian Novello e Alessandro) un casamentum con edificio sovrastante posto in Modena in contrada de Ferariis nella porta di S. Pietro in pagamento di un debito di 40 lire modenesi contratto verso di essi, i quali avevano versato la somma a Maria, madre di Albertina. Albertina appartiene a una famiglia dell'aristocrazia rurale legata a un insediamento fortificato situato nella fascia appenninica della valle del fiume Panaro nel territorio della pieve di Trebbio: DTS II, p. 85.

94 ASMo, Memoriale antico, a. 1275, n. 2453 (12 agosto 1275): Niccolò del fu Giovannibuono Mattarelli acquista per la somma di 150 lire modenesi dai frati Eremitani duo casamenta pro parte vacuis et pro parte edificatis situati in Modena in porta Baggiovara nella cinquantina di S. Barnaba, confinanti con la strada della contrada e con la proprietà di Guglielmo Adelardi, il quale acconsente alla vendita e rinuncia a ogni diritto sui beni ceduti. Anche per Murano, Copisti a Bologna, p. 32 , nota 106, il contratto dà prova delle ampie disponibilità economiche di Mattarelli. Le cinquantine, che alla fine del Duecento ammontano al numero di 30 interne e 9 esterne al perimetro cittadino, prendono solitamente nome dalla chiesa o da una delle chiese poste al loro interno tranne quelle ubicate lungo il perimetro della città in corrispondenza delle porte e quelle che, prive di chiese, traggono il nome da un altro elemento di significato particolare per la comunità locale o riconducibile a un uso tradizionale in rapporto alla fisionomia di ciascuna di esse: su questo si veda RöLKer, Nobiltà e Comune a Modena, p. 240 s.; BonaCinI, Il Registrum Comunis Mutine, p. 71 ss., 97 s., 115 ss. 
lazzi del Comune e la cattedrale, Niccolò Mattarelli consolida una dotazione immobiliare frutto di acquisizioni realizzate sia assieme al fratello Gian Novello, sia a titolo personale. Nel marzo 1280, infatti, egli acquista per la notevole somma di 300 lire modenesi una casa posta nella contrada del Castellaro, che ancora oggi sbocca direttamente sulla piazza allineandosi parallelamente alla via Emilia ${ }^{95}$; l'immobile risulta contiguo a un altro di cui sono già titolari Niccolò e il fratello Gian Novello, i quali due mesi più tardi procedono alla divisione legale della proprietà ${ }^{96}$. Sulla casa acquistata da Niccolò in contrada del Castellaro sembrano gravare diritti in quale misura riconducibili all'ordine dei Templari, documentato a Modena dal 1176 e in seguito nel contado, forse come effetto di donazioni disposte in favore di quest'ultimo dai precedenti proprietari. In ogni caso, al fine di tutelare l'acquisto effettuato nel 1280 dal doctor legum, molti anni più tardi, nel 1307, il praeceptor domus militie Templi de Mutina conferma la vendita disposta 27 anni prima implicitamente rinunciando a ogni rivendicazione su di essa ${ }^{97}$.

Da tempo il medesimo responsabile della residenza modenese

95 ASMo, Memoriale antico, a. 1280, n. 3586 (5 marzo 1280): il dominus Nicolao de Matarello acquista da Tomasina del fu Modenese Scudari e dal marito Giovanni da San Martino una casa ubicata nella contrada del Castellaro per la somma di 300 lire modenesi; il terreno, già edificato, confina su un lato con la strada suddetta, su altri due lati con proprietà dello stesso Niccolò e di uno dei fratelli, il magister Gian Novello. Per Murano, Copisti a Bologna, p. 32, nota 106, conferma ancora le ampie disponibilità economiche di Mattarelli. Sulla contrada e la sua denominazione di origine medievale si rinvia a VICINI, I confini della parrocchia del Duomo, p. $99 \mathrm{s.}$

96 ASMo, Memoriale antico, a. 1280, n. 4999 (6 maggio 1280); Niccolò e il fratello, il magister Gian Novello, figli del fu Giovannibuono Mattarelli, si dividono una casa di loro proprietà posta nella contrada del Castellaro contigua a quella già acquistata da Niccolò da Tomasina del fu Modenese Scudari.

97 ASMo, Memoriale antico, a. 1307/1, n. 1044 (11 aprile 1307): frate Pietro praeceptor domus militie Templi de Mutina conferma la vendita di una casa in Modena fatta al doctor legum Niccolò Mattarelli da Tomasina del fu Modenese di Enrico Scudari; fra i testi è presente il fratello Alessandro. È verosimile che le donazioni effettuate in favore dei Templari fossero successive al 1280 e che l'atto cui si giunge nel 1307 componga una vertenza sorta in relazione all'immobile acquistato dal giurista, rivendicato dai Templari in quanto attratto nel complessivo asse patrimoniale a loro devoluto da Tomasina del fu Modenese Scudari. Sull'insediamento dei Templari a Modena e nel territorio tra XII e XIV secolo, con particolare riferimento alla residenza e all'ospitale situato a est della città vicino all'attraversamento del fiume Panaro, si veda TRотA, L'ordine dei cavalieri templari a Modena, in part. p. 32 ss. per ulteriori fonti riguardanti il procuratore dell'ordine frate Pietro da Montecucco. 
dei Templari, il preceptor et procurator Pietro, aveva conoscenza diretta di Niccolò Mattarelli, giacché proprio nel 1280 il già rinomato legum doctor era stato scelto quale arbitro, assieme a Corradino de' Migliarini, in una vertenza tra lo stesso Pietro e i fedecommissari testamentari chiamati a dare esecuzione ai legati disposti dal fu Rainero da Castello98; inoltre nel marzo 1283 intervengono entrambi come testimoni a un atto di investitura feudale disposto dal vescovo di Modena Ardizzone ${ }^{99}$.

Ancora in contrada del Castellaro Niccolò Mattarelli incrementa il proprio patrimonio allargando il nucleo immobiliare già accumulato, certamente in accordo con il fratello Gian Novello, tramite l'acquisto nel 1289 di una casa collocata sul fianco della proprietà di quest'ultimo ${ }^{100}$.

\section{La committenza di manoscritti}

Un ulteriore profilo attraverso il quale si può valutare la disponibilità economica di cui gode Mattarelli tocca il piano della committenza di manoscritti a contenuto giuridico e scritturale che il maestro si procura avvalendosi di alcuni tra i più accreditati amanuensi attivi sul mercato librario modenese e bolognese.

98 ASMo, Memoriale antico, a. 1280, n. 2718 (16 febbraio 1280) il legum doctor Niccolò Mattarelli, assieme a Corradino de' Migliarini, è chiamato a fare da arbitro in una vertenza tra frate Pietro preceptor atque procurator domus milicie Templi de Mutina e i fedecommissari testamentari chiamati a dare esecuzione ai legati disposti dal fu Rainero da Castello, in merito a un lascito del valore di 25 lire modenesi; n. 2719: in pari data i due arbitri accettano di comporre la vertenza tra le parti suddette.

99 AIMAe, V, coll. 299-301. Vedi sotto nota 134.

100 ASMo, Memoriale antico, a. 1289, n. 1453 (18 agosto del 1289): Francesco Mattarelli, agendo per il padre assente, acquista da Ugolino del fu Martino Benassati per la somma di 51 lire modenesi una casa situata in Modena, in contrada del Castellaro, confinante anche con la proprietà di Gian Novello Mattarelli. Una conferma della concentrazione del patrimonio immobiliare di famiglia attorno a questo asse viario viene da un atto del 13 gennaio 1377 con cui Bitina de' Mattarelli vende agli eredi di Gentile, nipote di uno dei fratelli di Niccolò, una casa con cortile delimitata dalla medesima strada, da una piccola piazza pubblica e dalla contrada della carceri del Comune (VICINI, I confini della parrocchia del Duomo, p. 103). 
Il decennio che si avvia dai primi anni Settanta del Duecento durante il quale si può collocare il magistero modenese di Mattarelli, giurista ormai affermato in sede locale, coincide con «uno dei più favorevoli per la cultura cittadina, dimostrata viva dai numerosissimi contratti riguardanti trascrizioni di manoscritti, specialmente giuridici ${ }^{101}$. Da essi emerge anche l'interesse scientifico e l'impegno economico di Mattarelli, che per la realizzazione di codici sia a contenuto giuridico, da completare con l'apparato accursiano ormai ritenuto ordinario, sia a contenuto biblico incarica abili copisti già legati all'ambiente universitario delle due limitrofe città padane.

Il 28 giugno 1274 Niccolò Mattarelli incarica i due figli del fu Pietro da Ganaceto, Delacore e Delaito, il quale è anche notaio, come capita di frequente tra i copisti attivi a Modena e Bologna nel Duecento ${ }^{102}$, di scribere et glosare de aparaticu ordinario domini Acursi quemdam codicem scriptum in textu in cartis edinis dietro un compenso di 37 lire modenesi ${ }^{103}$. All'attenzione del maestro modenese è l'apparato al Codex, che assieme al Digesto vecchio costituisce il pilastro delle letture ordinarie, predisposto verosimilmente da Accursio in un lasso di tempo compreso tra i 40 e i 50 anni prima e già considerato come ordinarius dai giuristi più giovani e attivi anche al di fuori dello Studio felsineo ${ }^{104}$.

101 Mor-Di PIetro, Storia dell'Università di Modena, I, p. 12, con riferimento a SANDONNINI, Di un codice, pp. 122-129, ove sono trascritti i sunti di 33 contratti relativi alla compravendita oppure alla scrittura di codici (tratti da ASMo, Memoriale antico), molti dei quali verranno citati nelle note seguenti.

102 Soetermeer, A propos d'une famille de copistes, p. 433 ss.; ID., Exemplar und Pecia, p. 494 ss.

103 ASMo, Memoriale antico, a. 1274, n. 4663, trascritto in SANDONNINI, Di un codice, p. 123. Lo ricorda Murano, Copisti a Bologna, p. 32 e nota 107, come testimonianza delle ampie disponibilità economiche di Mattarelli, che si manifestano anche nel conferimento di diversi incarichi di copiatura di codici. Delaito è registrato nella matricola dei notai modenesi: Vicini, Statuti, p. 92.

104 Secondo una notizia riferita da Angelo degli Ubaldi sullo scorcio del Trecento, il Codex sarebbe stata la prima parte dei testi giustinianei a essere completata da Accursio con il proprio apparato, seguita poi dal Digesto vecchio e quindi dal Digesto nuovo, dall'Inforziato e dalle Istituzioni: VALENTINI, L'ordine degli apparati accursiani, in part. p. 110 per la possibile datazione della Glossa al Digesto vecchio, in base a considerazioni di Giovanni Gualandi, circa tra la metà degli anni Venti e la metà degli anni Trenta del Duecento. La rapida egemonia acquisita dall'apparato accursiano sul mercato della cultura e del libro giuridico può essere stata favorita dal complesso di attività esercitate dal maestro e dai figli, presenti nel- 
Il 15 settembre sempre del 1274 il magister Bernardino Bochignolli, figlio di Giliolo, con il consenso e la volontà del padre, promette al doctor legum modenese di vergare di propria mano, in Modena o in Bologna, il testo di una parte della Bibbia al costo di 40 lire modenesi ${ }^{105}$. Si tratta di uno dei non pochi copisti attivi sulle due piazze di Bologna e Modena nella fitta attività di riproduzione di testi in prevalenza giuridici, e tra essi Bernardino soffre le pesanti conseguenze della guerra civile culminata nella città felsinea nell'aprile 1274 che, con l'affermazione violenta della parte guelfa capeggiata dai Geremei, provoca l'emigrazione anche di numerosi studenti e maestri, la contrazione del mercato librario di matrice universitaria e la parallela diaspora, per ragioni sia di opportunità professionale che di appartenenza politica, di non pochi copisti ${ }^{106}$.

Nonostante tali fattori sfavorevoli, che certamente determinano riflessi problematici sulla committenza di manoscritti di pregio e sulla complessiva attività dei professionisti della scrittura di codici legati all'ambiente universitario, l'opera di Bernardino ha potuto esprimersi in un esemplare prezioso e di notevole rilievo, se è possibile identificare quest'ultimo - alla luce delle ricerche di Giovanna Murano e Mas-

lo spazio della città e dello Studio felsineo non soltanto come autori e docenti, ma anche come proprietari per decenni «di una fiorente statio di riproduzione peciaria»: Menzinger-Vallerani, Giuristi e città, p. 211.

105 ASMo, Memoriale antico, a. 1274, n. 1293, trascritto in SANDONNINI, Di un codice, p. 124. Si veda Murano, Copisti a Bologna, p. 31 ed Ead., Paolo di Jacopino Avvocati, p. 26, secondo la quale «la presenza del padre o di altro procuratore alla stipula dei contratti è necessaria, così come previsto dagli statuti, per i copisti che non hanno ancora raggiunto la maggiore età e per le donne; poiché nei precedenti contratti bolognesi Bernardino stipula a proprio nome è probabile che il padre sia stato nominato in questa occasione soltanto per dare maggior enfasi al documento». Bernardino, modenese di origine e anche notaio, risulta documentato tra 1268 e 1278 ed è uno degli oltre 270 copisti attivi a Bologna tra 1265 e 1270: per notizie sulla sua attività scrittoria e per il complesso delle testimonianze manoscritte che lo riguardano si veda SOETERMEER, The origins of ms. d'Ablaing 14, p. 106, nota 28; ID., Exemplar und Pecia, p. 496; Murano, Copisti a Bologna, p. 109 s. In Modena lo stesso copista, sempre nel 1274, sottoscrive un contratto per finire unum suum [del committente] codicem e per realizzare sia il testo delle Decretali sia della Summa Codicis di Azzone; quindi nel 1276 incarica tre altri copisti di facere et complere et ei dare unum codicem in cartis edinis formato da 19 quinterni dietro compenso di 42 lire modenesi (ASMo, Memoriale antico, a. 1274, n. 3935 e a. 1276, n. 3862, trascritti in SANDONNINI, Di un codice, pp. 123, 125).

106 Soetermeer, The origins of ms. d'Ablaing 14, p. 103 s.; ID., Utrumque ius in peciis, p. 206. 
simo Medica - con la notissima Bibbia sottoscritta di proprio pugno dal magister modenese, attualmente conservata nella Biblioteca della cattedrale di Girona e ritenuta «uno dei massimi capolavori della miniatura europea del Duecento» ${ }^{107}$.

La breve serie di contratti di scrittura sottoscritti da Mattarelli si conclude il 22 marzo 1283, quando il doctor iuris promette di corrispondere la cifra di 16 soldi modenesi pro quolibet quaterno stationis a Petrus de Lusscho dietro assicurazione che il figlio di questi, Rolando, trascriverà le glosse al Digesto Vecchio in margine a un esemplare dello stesso Niccolò ${ }^{108}$.

Nello stesso torno di tempo, fra gli anni Settanta e Ottanta del Duecento, la riproduzione del manoscritto giuridico è un'attività ben documentata a Modena e interessa un ventaglio assai ampio e diversificato di testi seguendo con precisione, in alcuni casi, anche il sistema di tassazione praticato nelle botteghe dei copisti bolognesi ${ }^{109}$.

107 Murano, Paolo di Jacopino Avvocati, p. 27 s.; Ead., Firme e monogrammi d'artista, p. 76. Nel caso di specie Giovanna Murano suppone che Mattarelli abbia commissionato la Bibbia a Bernardino Bochignolli per conto di Jean Cholet (1212/1222-1292), doctor in utroque a Parigi e Orléans ed elevato alla porpora cardinalizia nel 1281, il quale secondo MedicA, La Bibbia di Gerona, p. 72 per la citazione e p. 78 per la sottoscrizione del noto copista Bernardino da Modena, sarebbe stato il committente effettivo dell'opera (Biblioteca Capitolare di Girona, ms. 10 [1-2], fine XIII sec.), acquistata dal re di Francia Carlo V nel 1378 presso l'abbazia di Saint-Lucien de Beauvais e miniata in un atelier bolognese da cui uscirono numerose altre Bibbie illustrate in modo analogo.

108 ASMo, Memoriale antico, a. 1283, n. 3878, ricordato in VicinI, Di Nicolò Mattarelli, p. 15, il quale legge il nome del sottoscrittore come Pietro del Bosco. Nel contratto si precisa che il primo quaterno termina con la parola «villis», mentre il secondo quaterno inizia con la parola «monimentis» e termina con la parola «renuntiaverit». Il contratto sottoscritto da Mattarelli è ricordato anche in SOETERMEER, Utrumque ius in peciis, p. 127, a proposito del sistema di tassazione 'per quaterni', praticato con evidenza anche a Modena, come confermano alcuni contratti di scrittura risalenti al 1279 citati sotto, nota 112. A titolo di ampio confronto, nel corso del Duecento a Bologna il prezzo di un intero esemplare manoscritto del Digesto vecchio aumenta dalle 20 (corrispondenti a 10 lire imperiali) a 40 lire di bolognini, mentre un analogo esemplare dotato di apparato accursiano raggiunge le 50 lire di bolognini: GRECI, Note sul commercio del libro universitario, p. 51 ss.

109 È accertato che il sistema di tassazione praticato a Bologna nella riproduzione del libro giuridico mediante exemplaria suddivisi in quaterni, formati da due pecie, e depositati presso le stationes appositamente autorizzate viene impiegato anche a Modena, ove trova una chiara benché tardiva sanzione normativa negli Statuti cittadini del 1327: Statuta civitatis Mutine, 1. I, r. CLXIII, p. 162 s. (Quod unus stationarius habeatur qui habeat esempla in iure civili et canonico), riprodotta in SANDON- 
Nel 1279 il facoltoso canonico di Reims Guglielmo, appartenente all'ordine dei Predicatori, originario di Laon e in seguito nominato nel 1314 docente di teologia da papa Clemente $\mathrm{V}^{110}$, si procura copie di numerosi testi civilistici e canonistici comprensivi dei rispettivi apparati di glosse, che in genere presentano un costo inferiore a quello del testo giuridico cui si riferiscono: un esemplare, rispettivamente, del Codice, del Digesto vecchio e delle Decretali, un esemplare dell'Inforziato cum trespartu ${ }^{111}$ e un esemplare del Volumen che include, come viene specificato nel contratto di scrittura, tres libros codicis et institutiones et autenticum et ius feudorum ${ }^{112}$.

Negli anni successivi, tra 1280 e 1282, lo stesso ecclesiastico si provvede di un'ulteriore copia del Codice, del Digesto vecchio e delle Decretali, di un esemplare della summa domini Açonis cum ordinariis et extraordinariis et cum additionibus e della glossa al Decre-

NinI, Di un codice, p. 117 s. Si veda Soetermeer, Utrumque ius in peciis, p. 69 ss., p. 117 ss., p. 127 (ove si ricorda l'incarico di copiatura conferito da Mattarelli nel marzo 1283), p. 133 ss. e p. 205. Si rammenti che gli Statuti cittadini riformati nel 1327 durante un breve periodo di governo del legato pontificio Bertrando del Poggetto costituiscono la prima redazione organica a noi giunta, ma proprio per tale ragione contenente materiale normativo derivato da redazioni precedenti e non conservate nella loro integrità; cfr. BonacinI, $I l$ Registrum Comunis Mutine, p. 28 ss.

110 Regestum Clementis Papae V, IX, Romae 1888, p. 89 s., nn. 10.28510.286, apud Castrumnovum Avinionensis diocesis, 1314 marzo 29; ricordato in SANDONnINI, Di un codice, p. 100, nota 1.

111 Nella produzione del manoscritto giuridico ancora del tardo Duecento le sezioni finali dell'Inforziato, le cosiddette tres partes, forse perché appartenenti in origine al Digesto nuovo, sono oggetto di una specifica divisione rispetto al testo precedente e di un trattamento differenziato da parte dei copisti, laddove, in numerosi manoscritti risalenti al XII e al XIII secolo, esse erano scritte da una mano diversa da quella che si applicava sui restanti libri centrali del Digesto: si veda in merito l'ampia e documentata ricerca di VAN DE Wouw, Zur Textgeschichte des Infortiatum. Altri contratti di scrittura modenesi esplicitano la riproduzione manoscritta dell'Inforziato tanto cum tribus partibus quanto sine tribus partibus: ASMo, Memoriale antico, a. 1275, n. 4365; a. 1279, n. 972, con trascrizione in SANDONNINI, Di un codice, p. 125 s.

112 ASMo, Memoriale antico, a. 1279, nn. 1020 e 1021: si riferiscono alla registrazione complessiva di sei contratti di scrittura datati al 19 dicembre, con trascrizione in Sandonnini, Di un codice, p. $126 \mathrm{~s}$. e in Soetermeer, A propos d'une famille de copistes, IV, p. 472. Secondo l'ultima registrazione, relativa alla scrittura del testo di un Codice, un Digesto vecchio e un esemplare delle Decretali, il committente si impegna a pagare al copista 25 soldi modenesi pro quolibet quaterno, taxati Bononie; anche un'altra tra le precedenti committenze, relativa alla glossatura di un Volumen, viene remunerata in base a quolibet quaterno taxato. Si veda in merito ID., A propos d'une famille de copistes, p. 451 e nota 123, e ID., Exemplar und Pecia, p. 489. 
tum $^{113}$, e infine acquista una casa in città da Francesco di Grasolfo, notaio e a sua volta appartenente a una famiglia di specialisti nella riproduzione manoscritta dei testi giuridici ${ }^{114}$.

Considerato lo stato ecclesiastico di Guglielmo, unito a un forte interesse verso i testi canonistici ma integrato dalla conoscenza di quelli civilistici e della consolidata dottrina imperniata sulla Glossa e sulla Summa azzoniana, e pure dalla contestuale necessità di acquisire una stabile dimora in Modena, non è escluso che egli in seguito divenga «uno dei Lettori dello Studio modenese, riprendendo l'insegnamento del diritto canonico, che un decennio prima o poco più aveva avviato l'altro grande canonista francese Guglielmo Durante» ${ }^{15}$.

\section{Al governo del Comune}

Nell'arco di oltre un trentennio, compreso tra gli anni Settanta del Duecento e il primo decennio del secolo seguente, Niccolò Mattarelli

113 ASMo, Memoriale antico, a. 1280, n. 297; a. 1281, n. 337; a. 1282, n. 3565, con trascrizione in Sandonnini, Di un codice, p. 127 s. e in Soetermeer, $A$ propos d'une famille de copistes, V e VII, p. $472 \mathrm{~s}$.

114 ASMo, Memoriale antico, a. 1282, n. 3566, con trascrizione in SANDONNINI, Di un codice, p. 128 e in Soetermeer, A propos d'une famille de copistes, VIII, p. 473. Sul notaio-copista Francesco di Grasolfo si rinvia a ID., A propos d'une famille de copistes, in specie p. 457 ss. Sempre a Modena lo stesso Francesco di Grasolfo si impegna a realizzare l'apparato all'Inforziato nel 1273 e sei anni più tardi il testo delle Decretali, mentre nel 1281 suo fratello Iacopo promette, a nome di un altro fratello, di scrivere la glossa al Decretum: ASMo, Memoriale antico, a. 1273, n. 4622; a. 1279 , n. 2481; a. 1281, n. 3508, con trascrizione in SANDONNINI, Di un codice, pp. 123, 127, 128, e in SOETERMEer, A propos d'une famille de copistes, II e III, p. 472, VI, p. 473, e cfr. pure p. 449. Ancora nel 1273 Bonfigliolo del fu Guidotto da Marano promette al padre provinciale Pietro Bernardo de Cedollis di glosare ei Decretum de glosiis consuetis et exemplare bene ponendo gloxas locis congruis sua propria manu ordinate: ASMo, Memoriale antico, a. 1274, n. 3984, con trascrizione in SANDONNINI, Di un codice, p. 123.

115 Vicin, Profilo storico, p. 17. Diversamente Murano, Firme e monogrammi d'artista, p. 81, nota 19, ritiene che Guglielmo di Laon stipuli contratti di scrittura non per esigenze personali, ma «per conto di ricchi committenti o istituzioni d'Oltralpe» e che la medesima funzione di procuratore per conto terzi sia svolta da Niccolò Mattarelli nel momento in cui stipula onerosi contratti di scrittura per copie del Codice e del Digesto vecchio. Ma ciò contrasta con il contratto relativo alla scrittura della Glossa al Digesto vecchio su un esemplare di proprietà dello stesso maestro. 
appare in relazione assai ravvicinata e, in certe fasi, direttamente organica alle istituzioni comunali modenesi in una misura che travalica il prestigio del proprio ruolo e la competenza tecnica di cui è portatore e ne fa un protagonista attivo e diretto degli organi di governo della città nel periodo successivo al 1271, caratterizzato dalla rinnovata affermazione della parte popolare al vertice del Comune modenese.

In quell'anno, infatti, dopo una fase di prevalenza dell'aristocrazia cittadina di parte guelfa che si riconosceva nella fazione degli Aigoni, le forze popolari raggiungono l'obiettivo di costituire un'organizzazione politica separata e alternativa rispetto a quella del Comune di matrice aristocratica tramite la formazione di una unitaria Societas Populi, che pare dotata di una propria configurazione militare e che trova un proprio spazio di solida rappresentanza politica nella nuova magistratura del Capitano del Popolo, in carica per sei mesi affiancato da un giudice, e nella creazione di un consiglio di 24 Defensores populi sempre espressione delle forze popolari ${ }^{116}$. A quello stesso anno appartiene anche la prima testimonianza relativa all'esistenza di statuta et ordinamenta et decreta populi, ossia di uno specifico statuto della parte popolare di cui si ha notizia indiretta ancora pochi anni dopo, nel $1276^{117}$.

Si tratta di un assetto istituzionale che ripropone, con forti analogie, quello già collaudato alcuni anni prima, nel quindicennio compreso tra 1249 e 1264, quando la colonizzazione politica del vicino comune di Bologna conseguente alla pesante sconfitta militare subita da Modena aveva favorito la creazione della nuova magistratura degli Anziani, destinata a riunire 12 delegati della parte popolare quali rappresentanti dell'esecutivo cittadino accanto al podestà, in forte prevalenza di estrazione bolognese e, di fatto, posto al vertice del Comune in cui si riconoscevano i due differenti e contrapposti schieramenti dell'aristocrazia cittadina ${ }^{118}$.

Sullo sfondo del nuovo scenario politico che si avvia dal 1271, quando il populus governa la città in condominio con la sola fazione

116 Simeoni, Ricerche sulle origini, p. 158 ss.; Vicini, I Capitani del Popolo, p. 198; SAntinI, Università e società, p. 344; Rölker, Nobiltà e Comune a Modena, p. 240 ss.; Bonacini, Dinamiche istituzionali, p. 431 ss.

117 Simeoni, Ricerche sulle origini, p. $159 \mathrm{~s}$.

118 RölKer, Nobiltà e comune a Modena, p. 237 ss.; BonacinI, Dinamiche istituzionali, p. 428 ss. e p. 455. 
dell'aristocrazia urbana che si identifica nel più ampio schieramento guelfo e che su base locale continua a essere rappresentata dal Podestà, Niccolò Mattarelli partecipa ad atti importanti della vita istituzionale cittadina ricoprendo anche, in alcuni casi, specifici incarichi pubblici.

Nelle vesti di testimone ad atti rilevanti che in forme diverse coinvolgono il Comune di Modena egli è presente in palazzo comunale dal maggio 1272, già qualificato dal titolo di doctor legum ${ }^{119}$, e nel medesimo ruolo interviene più volte ai giuramenti prestati in pleno consilio generali communis Mutine da Pellegrino Guidoni, da Grassone Grassoni e da suo cugino Bernardino Grassoni al momento di accettare, rispettivamente, la nomina a Capitano del Popolo di Perugia ${ }^{120}$ e di Cremona ${ }^{121}$ e di Podestà di Volterra ${ }^{122}$.

119 ASMo, Memoriale antico, a. 1272/1, n. 497 (14 maggio 1272): in presenza di Nicholao de Mattarelli doctore legum e di altri testimoni, nel palazzo comunale di Modena il procuratore del Comune di San Felice Guido de Husbertis vende ad Armanino e Gerardino Munari una porzione di terreno comunale nella corte di S. Felice detta bosco di Marzana, confinante sul lato orientale con il corso del Naviglio del Comune di Modena, per la somma di 500 lire modenesi. Gli altri testimoni presenti (Coradino Armanini de Miliarinis, Petro Roberti, Oprandino de Solaria et Philippo domini Petri de Rodelia) sono identificati da VIcINI, Di Nicolò Mattarelli, p. 10, come «vari giudici». ASMo, Memoriale antico, a. 1280, n. 1414 (1 agosto 1280): il legum doctor Nicholaus Mattarelli, insieme con Tommaso da Spezzano e Corradino de' Migliarini, nel Palazzo nuovo del Comune di Modena testimonia all'atto con cui Gerardo da Costrignano, sindaco del Comune, promette di pagare 40 lire modenesi al legum doctor Guido da Suzzara assolvendolo da ogni obbligazione verso il Comune stesso.

120 ASMo, Memoriale antico, a. 1272/1, n. 1410 (26 ottobre 1272): Nicholao de Matarello legum professor testimonia al giuramento che presta in pieno Consiglio, di fronte pure ai sindaci del Comune di Perugia, Pellegrino de Guidonibus, nominato Capitano del Popolo di Perugia.

121 ASMo, Memoriale antico, a. 1275, n. 3454 (26 giugno 1275): Nicholao Matharelli testimonia all'atto solenne con cui Garxonus de' Garxonis in pleno consilio generali communis Mutinae accetta e giura il capitaneatum populi Cremonae dietro corresponsione di un salario di 300 lire imperiali. Addobbato cavaliere nel 1270 assieme al cugino Bernardino dall'imperatore Michele Paleologo di passaggio a Modena, Grassone Grassoni, esponente di una cospicua famiglia di parte guelfa, esercita pure l'ufficio di capitano del Popolo a Perugia nel 1271 e in seguito di podestà a Parma e Lucca nel 1282 e nel 1284: LoRENZONI, Prime ricerche sulla famiglia Grassoni, p. 220 ss.

122 ASMo, Memoriale antico, a. 1279, n. 1727 e n. 1728 (14 novembre 1279): il dominus Nicholaus Matarello testimonia a Modena, assieme ad altri, all'atto con cui Bernardino de Garxonibus è nominato podestà di Volterra dai procuratori di questa città con il salario di 600 lire di pisani piccoli; ed è presente anche al conseguente giuramento da parte del podestà appena nominato. Con uno scarto sia onomastico che cronologico, Cecina, Notizie istoriche della città di Volterra, p. 261, 
Lo scambio di ufficiali incaricati di svolgere il mandato di podestà o di capitano del Popolo assume un fondamentale significato nel corso del Duecento per il consolidamento di alleanze politiche intercittadine che travalicano la fase di più rigida suddivisione in schieramenti orientati a sostenere oppure a rifiutare, nel corso del secondo quarto del secolo, la politica egemonica impostata da Federico II e fortemente osteggiata dal papato. Nei decenni successivi tali alleanze si rimodulano secondo un'articolazione in partes di matrice aristocratica schierate con la Chiesa o con l'Impero, che si mostrano variamente disponibili a convergenze e sperimentazioni politiche, su base locale, con le forze popolari e che soltanto dagli inizi del Trecento iniziano a distinguersi con precisione assumendo ovunque e in forma generalizzata i nomi distintivi di guelfi e ghibellini ${ }^{123}$.

Nell'esperienza modenese che vede il coinvolgimento diretto di Mattarelli è significativo il legame con Cremona, ove dal 1271 si installa al potere per un quarantennio una diarchia formata dal Popolo e dalla parte guelfa che scambia ufficiali in larga misura con città padane tradizionalmente alleate, come Modena, e collocate nel raggio di un centinaio di chilometri di distanza ${ }^{124}$. Si configura quindi un assetto politico analogo a quello maturato dallo stesso anno in quest'ultima città che motiva l'orientamento allo scambio di ufficiali tra i due centri urbani, dal momento che Modena, da cui proviene Grassone Grassoni per assumere la carica di Capitano del Popolo a Cremona nel 1275, riceve podestà cremonesi nel secondo semestre del 1273 (Guglielmo Oldoini), nel primo semestre del 1277 (Giovanni da Piscarolo) e ancora nel I semestre del 1283 (Guidotto Arcidiaconi $)^{125}$. E quattro anni più tardi riceve anche un Capitano

assegna la podesteria di Bernardinus de Opizzonibus de Mutina al secondo semestre dello stesso 1279 , nel complesso confermando sulla base delle fonti municipali l'ufficio podestarile svolto da un modenese nella città toscana. Nel passato Volterra avrebbe ricevuto un solo altro ufficiale modenese, Raynerius Pance de Buccabadatis de Mutina, nel primo semestre del 1252 (ibidem, p. 259).

123 Sul punto, le sintesi più recenti ed efficaci si devono a Milani, I comuni italiani, p. 113 ss. e a Menant, L'Italia dei comuni, p. 77 ss. Sulla specifica situazione modenese si rinvia a Bonacin, Dinamiche istituzionali, p. 428 ss., 453 ss.

124 Menant, Podestats et capitaines du peuple, p. 79 s., 84 ss.

125 Bonacins, Dinamiche istituzionali, Appendice IV, p. 483 s. 
del Popolo cremonese nella persona di Niccolò di Oddone Cortesi, in carica a Modena durante il I semestre del $1287^{126}$.

Un impegno diretto di Mattarelli nell'attività di governo emerge nel 1279, quando il dominus partecipa all'organo collegiale dei 24 Difensori del Popolo, che rappresenta a livello politico tale raggruppamento della società urbana svolgendo un ruolo tanto consultivo quanto esecutivo al fianco del Capitano del Popolo. In quell'ufficio Mattarelli assume una obbligazione in solido per l'alta cifra di 800 lire modenesi assieme a numerosi altri soggetti coobbligati che sembra fungere da garanzia rispetto a un debito contratto dal Comune di Modena nei confronti di cittadini fiorentini ${ }^{127}$.

Il forte impegno economico assunto personalmente da Mattarelli in quanto membro di un organo di governo si giustifica nel contesto delle pesanti sanzioni ecclesiastiche e finanziarie inflitte al Comune di Modena dal legato apostolico in seguito alla redazione di statuti ritenuti contrari alle libertà ecclesiastiche nonché per soprusi e danni inferti dal Comune stesso al monastero benedettino di Nonantola e ai coltivatori delle sue terre, per i quali erano stati comminati ai modenesi scomunica e interdetto, oltre al pagamento di 370 lire a titolo di indennizzo al cenobio e di altre 1.000 lire bolognesi a titolo di multa ${ }^{128}$.

A causa di queste iniziative messe in atto dalle autorità comunali papa Nicolò III aveva scomunicato queste ultime e lanciato l'interdetto sulla città attraverso il cardinale Latino Malabranca, legato

126 Vicini, I Capitani del Popolo, p. 181.

127 ASMo, Memoriale antico, a. 1279, n. 2697 (31 ottobre 1279): il dominus Nicholao de Matarelo, Filippo degli Occhi, Andrea Donolina, Francesco Merzadro, Amadeo de Veneciis, Bonincontro Spingardo, Zunta de Moza, Odorico Trenti, Guiberto calegario, Ugolino qui non sudat, Iacopino de Bozalibus, Modenese Purzilini, Giovanni del Ferro, Ambrogio da Imola, Ricobono de' Tregesi, Tommaso Pizoni, Paganino Cesi, Rolandino campanario, Altemanno tuscho, Iacopo de Tadulinis e Siletto Petrezzani sono assolti da Pietrobono del fu Ianuario, sindaco e procuratore del Comune di Modena, da una obbligazione di 800 lire modenesi che essi avevano contratto verso Spino de Nebola da Firenze e altri soggetti per il Comune stesso. VICINI, Di Niccolò Mattarelli, p. 12, ritiene assai probabile che il giurista abbia ricoperto in quell'anno l'ufficio di Difensore del Popolo, «anzi si può dire che è certo», giacché il suo nome risulta affiancato a coloro che, in base ad altre fonti, ricoprivano con certezza la medesima carica.

128 MSM II, p. 100 s.; SBN I, p. 137 ss.; VICINI, Di Niccolò Mattarelli, p. 12 s. 
apostolico in Romagna e in Toscana tra 1278 e 1280 con l'obiettivo principale di sviluppare un'opera di mediazione e pacificazione soprattutto a Bologna e a Firenze in funzione antiangioina e di consolidare i possessi della S. Sede in Romagna e nelle Marche ${ }^{129}$.

Come comprova un fascio di atti redatti nel cuore della città, presso le residenze delle autorità laiche ed ecclesiastiche coinvolte nella controversia - il palazzo vescovile, la cattedrale intitolata a San Geminiano, il palazzo comunale -, e trascritti all'interno di uno dei due Libri Iurium fatti redigere dal Comune modenese nel secolo XIII, le multe inflitte vengono prontamente pagate nel novembre 1279. Fra i testimoni, sempre in posizione rilevante sia per ruolo istituzionale che per distinzione professionale, compare regolarmente il doctor legum Niccolò Mattarelli ${ }^{130}$ che continua l'attività pubblica anche l'anno successivo, quando viene eletto tra i Sapientes del Comune di Modena per il quartiere di porta S. Pietro ${ }^{131}$, lo

129 Tilatti, «Legatus de latere domini pape». Sullo specifico intervento nei confronti del governo fiorentino da parte del cardinal Latino, il cui padre aveva ricoperto l'ufficio podestarile nella medesima città nel 1238, si veda NAJEMY, Storia di Firenze, 1200-1575, p. 91 ss. Sull'operato dei legati pontifici nell'Italia centro-settentrionale nel corso del Duecento si veda anche MaIRE Vigueur, Religione e politica nella propaganda pontificia e TILATTI, Legati del papa e propaganda nel Duecento.

130 ASCMo, Camera Segreta, Registrum Antiquum, n. 491 (= ASMo, Memoriale antico, a. 1279, n. 2808-11 novembre 1279): in palatio episcopatus Mutine in presenza dei testimoni dominus Bonincontro archipresbitero maioris ecclesie Mutine, Coradino de Milliarinis, Nicholao de Matarelis, Filipo de Occulis, Iohanne ferario de Ganaceto notario, Lodoyco de Lodoycis, Ugolino de Ançillino et Alberto de Montis; ASCMo, Camera Segreta, Registrum Antiquum, n. 492 (= ASMo, Memoriale antico, a. 1279 , n. 2809-12 novembre 1279): in palatio comunis Mutine in pleno conscilio generali comunis et populi Mutine in presenza dei testimoni dominus Nicholao de Matarello, Gerardo Carepto, Nicholao de Rebusatis, Gratiadeo Andriani, Filipo de Oculis, Pasqua scudario; ASCMo, Camera Segreta, Registrum Antiquum, n. 493 (= ASMo, Memoriale antico, a. 1279, n. 2810-12 novembre 1279): in civitate Mutine iuxta ecclesiam beatissimi Geminiani confessoris in presenza come testimoni dell'abate del monastero di S. Pietro Simone, dell'arciprete della pieve di Cittanova, di Bonincontro arciprete della chiesa maggiore di Modena, del domino Nicholao de Matharello doctore legum, domino Iohanne ferario, domino Pasqua scudario, domino Anthonio de Rubeis.

131 ASCMo, Camera Segreta, Registrum Antiquum, n. 508 (24 luglio 1280): nell'atto, che riporta i nomi di tutti i Sapienti del Comune di Modena in carica per quell'anno, dominus Nicholaus Mattarelli è registrato, primo della lista, fra i $15 \mathrm{Sa}$ pienti per la porta di S. Pietro. Se ne contano 12 per porta Albareto, 10 per porta Cittanova (il primo è il notaio Nicolò de Brunecta) e 12 per porta Baggiovara. La 
stesso ove appare radicato tramite la proprietà di diverse unità immobiliari $^{132}$.

Le sanzioni ecclesiastiche comminate dal cardinal Latino sono revocate soltanto nel 1283 dopo che le autorità locali si sono rassegnate a delere de libro Statutorum Comunis Mutine omnia Statuta et provisiones contra libertatem Ecclesie accogliendo i moniti intimati in modo perentorio dal vescovo Ardizzone de' Conti (1282-87) tra i mesi di maggio e giugno dello stesso anno agli statutari, al podestà, al capitano del Popolo, ai 24 Difensori del Popolo e infine anche al Consiglio generale della città ${ }^{133}$. Lo stesso vescovo non manca di invitare Mattarelli nel palazzo episcopale, nel marzo dello stesso anno, per testimoniare a un atto di investitura feudale disposto in favore dei figli ed eredi del fu Francesco da Balugola, ai quali egli conferma tutti i beni e diritti connessi alla loro posizione di vassalli episcopali e all'esercizio dell'ufficio di avvocazia, ovvero di procuratores curiae episcopatus Mutine, detenuto da quella famiglia da diverse generazioni ${ }^{134}$.

In seguito, durante il non breve periodo compreso fra gli ultimi mesi del 1283 e l'estate del 1290, quando il maestro modenese è attestato a Padova ${ }^{135}$, di lui si perdono le tracce dirette: non risiede più a Modena, poiché vari atti notarili vedono comparire come procuratori o come semplici esecutori in sua vece il fratello Gian Novello oppure il figlio Francesco ${ }^{136}$, e non affiorano testimonianze di

fonte è ricordata pure in SANTINI, Università e società a Modena, p. 346. In seguito alla rivoluzione popolare che si verifica nel gennaio 1306 viene costituito un vero e proprio collegio formato da 40 sapientes de vero popullo civitatis Mutine, scelti dal Capitano del Popolo e deputati a provvedere circa reformationem et conservationem et bonum statum comunis et populli Mutine (RM I, p. 14).

132 Immobili situati nella contrada de Feraris e in quella del Castellaro: vedi sopra paragrafo 5 .

133 CAMPori, Intimazioni legali. Fasi e contenuti di questa tenace vertenza si possono ricostruire, per la maggior parte, attraverso un gruppo di pergamene conservate presso ACaMo, Diplomatico, pergamene O.15.670 (1283), O.19.690 (contenente tre atti del 1283), O.23.709 (contenente due atti del 1284).

134 AIMAe, V, coll. 299-301 (14 marzo 1283). Sulla potente famiglia da Balugola, originaria della montagna modenese e radicata con numerosi patrimoni fondiari in diverse località del contado, si veda RöLKER, Nobiltà e Comune a Modena, p. 52 ss. 135 Vedi cap. II, nota 7.

136 Posteriormente a un atto disposto il 17 agosto 1283 dal fratello Alessandro (ASMo, Memoriale antico, a. 1283, n. 1191), ove Niccolò Mattarelli interviene come testimone, si registra l'assenza di rogiti, nel periodo sopra indicato, in 
un suo eventuale insegnamento in sedi diverse da Modena né dello svolgimento di incarichi pubblici su invito di altre amministrazioni comunali, come l'ufficio di consultore ricoperto a Foligno nell'anno 1282 dietro un compenso di 400 lire cortones ${ }^{137}$.

Dal momento che Mattarelli risulta di nuovo presente nella propria città natale soltanto nell'agosto $1293^{138}$, si può supporre che il lungo periodo di assenza sia stato determinato da circostanze legate all'assetto politico della città, forse come effetto di un bando o una condanna comminata al giurista dal nuovo regime signorile avviato da Obizzo II d'Este dagli inizi del 1289, segnato dalla nomina di

grado di documentarne la presenza a Modena. Si veda ASMo, Memoriale antico, a. 1285, n. 533 (21 febbraio 1285): Tomasino Ungarini promette a Gian Novello Mattarelli, in qualità di procuratore del fratello Niccolò, di accettare 67 pecore in soccida dalla festa di tutti i Santi per 3 anni; ASMo, Memoriale antico, a. 1285, n. 211 (16 marzo 1285): Gian Novello Mattarelli consegna 40 lire modenesi alla moglie del fu Francesco Speciali, la quale agisce tutorio nomine in vece dei figli, in pagamento di un debito contratto dal fratello Niccolò; ASMo, Memoriale antico, a. 1288-89/1, n. 952 (20 aprile 1289): Francesco figlio Nicholay Matharel$l i$, quale procuratore del padre, acquista da Gerardo de Augustis e dai suoi figli Martino e Gianbuono iure libelli 3 appezzamenti di terreno situati nel distretto di Villavara e nella curia di Nonantola per la somma di 22 lire, 11 soldi e 1 denaro; ASMo, Memoriale Antico, a. 1288-89/1, n. 493 (20 agosto 1289): Giordano Caxarius promette ad Alessandro de Mattarellis una somma di denaro a titolo di mutuo. Tra i testimoni il fratello Gian Novello e Zanilino becario; ASMo, Memoriale antico, a. 1289, n. 1453 (18 agosto del 1289): vedi sopra nota 100; ASMo, Memoriale antico, a. 1291, n. 4136 (1 dicembre 1291): Francesco Mattarelli, sempre come procuratore del padre, permuta con Giliolo de' Canonici alcune terre ubicate nel distretto di Villavara.

137 ASMo, Memoriale antico, a. 1281, n. 3090 (24 novembre 1281): Nycholaus Mattharellus doctor legum accetta l'ufficio di consultore che gli viene offerto dai delegati della città di Foligno, fra' Bartolomeo priore dei Servi di Maria del beato Iacopo da Foligno, inviato a Modena come procuratore del Comune umbro assieme ad altri frati del medesimo ordine, con lo stipendio di 400 lire cortonesi. Dell'incarico svolto da Mattarelli non danno notizia i Fragmenta Fulginatis Historiae ab anno 1198 usque ad anno 1341, in AIMAe IV, coll. 137-148, strutturati in forma di scarno elenco dei podestà succedutisi a Foligno nel periodo sopra indicato integrato da brevi notazioni sugli avvenimenti politico-militari più significativi per la storia della città. Una decina di anni dopo la presenza di Mattarelli a Foligno l'ufficio podestarile viene attribuito a un altro modenese, Gerardo Bozzalini, che rimane in carica nel biennio 1292-93 (ibidem, col. 141).

138 ASMo, Memoriale antico, a. 1293, n. 2831 (19 agosto 1293): il dominus Nicholaus de Mattarellis legum doctor pro vicinia S. Laurentii promette di corrispondere a Guglielmo de Petreçanis ad eius voluntas la somma di 150 lire modenesi ex causa mutui supena dupli. 
un podestà di propria fiducia e dalla pronta abolizione delle magistrature popolari, Capitano del Popolo e 24 Difensori, nonché della Società e del Consiglio del Popolo ${ }^{139}$. Diversamente potrebbe essere anche conseguenza dei rapporti intessuti da Mattarelli con famiglie dell'aristocrazia cittadina che già pochi anni prima della presa del potere da parte del signore di Ferrara vengono espulse da Modena a causa della spaccatura avvenuta nel 1284 all'interno dello schieramento di matrice guelfa. In seguito agli squilibri che allora si erano determinati all'interno della parte aristocratica, la città era rimasta sotto il controllo delle famiglie Rangoni, Guidoni e Boschetti, e a quest'ultima apparteneva anche il vescovo Filippo, mentre veniva abbandonata dalle famiglie da Savignano, da Sassuolo e Grassoni assieme ai rispettivi alleati sia popolani che cavalieri, che avevano trovato rifugio in alcuni castelli del contado posti sulle prime colline a sud della città ${ }^{140}$.

La peculiarità della fase politico-istituzionale avviatasi a Modena dal 1271 e segnata dal condominio di potere tra Populus e aristocrazia cittadina di orientamento guelfo aveva certamente favorito l'avvicinamento di Mattarelli a diversi esponenti di spicco di questa parte della società urbana grazie anche, con larga probabilità, al prestigio professionale accumulato dal giurista. A livello pubblico nel corso degli anni Settanta egli è presente al conferimento di incarichi di Capitano del Popolo e di Podestà a membri delle famiglie Grassoni e Guidoni ${ }^{141}$, mentre a livello privato intesse rapporti negoziali e direttamente parentali con quest'ultima, in seguito al matrimonio del figlio Francesco con Giovanna Guidoni ${ }^{142}$, e sviluppa legami ugualmente stretti con Rangoni, Grassoni e Boschetti ${ }^{143}$. Dai Ran-

139 VICINI, I podestà di Modena, p. 180; ID., I Visconti Estensi, pp. 25, 31; Braidi-Tavilla, Statuti del Collegio dei giudici e degli avvocati, p. 48 ss.; Braidi, I Modenesi nel Trecento, p. $32 \mathrm{~s}$.

140 Bonacini, Dinamiche istituzionali, p. 433 s.

141 Vedi sopra note 120, 121, 122.

142 VicinI, Di Niccolò Mattarelli, p. 28. Vedi sopra, nota 92.

143 ASMo, Memoriale antico, a. 1280, n. 6182 (24 maggio 1280): Mattarelli, assieme a Girardo de Careptis, Guido Guidoni e Corradino da Savignano, testimonia alla promessa di matrimonio stipulata da Tobia Rangoni, per le due figlie Alda ed Erminia, rispettivamente con Guglielmo Grassoni e Nicolò Boschetti; Alda è destinata in sposa a Rodolfo figlio di Guglielmo Grassoni, Erminia a Dainesio figlio di Nicolò Boschetti. Secondo Vicini, Di Niccolò Mattarelli, p. 14, Corradino da Sa- 
goni gli viene pure affidato un arbitrato in merito a una controversia con un servitore di famiglia ${ }^{144}$.

Un segnale dell'emarginazione dalla vita politica cittadina di Mattarelli e della sua assenza da Modena nel periodo a cavallo tra gli anni Ottanta e Novanta del secolo XIII emerge sullo scorcio della vita del giurista, quando è già un affermato maestro presso lo Studio padovano. Nel settembre 1309, infatti, Tommasino del Ferro assolve Niccolò Mattarelli pro quadam securitate quod fecit pro dicto domino Nicholao penes Comunis Mutine sive marchionem estensem, ossia dal debito che il Maestro aveva contratto con lui in seguito a copertura della garanzia prestata in favore di Niccolò presso il Comune di Modena ovvero il marchese d'Este per la somma - comunque non eccessiva - di 25 lire modenesi. Mattarelli dal canto suo approva l'amministrazione dei propri beni fatta da Tommasino, che appartiene alla medesima pars Populi ${ }^{145}$ ed è in rapporti stretti con la famiglia del giurista anche per altri interessi ${ }^{146}$, assolvendolo da tutto ciò che egli potrebbe richiedere a lui in futuro in merito alla stessa amministrazione ${ }^{147}$.

vignano è un altro legum doctor mentre gli altri due testimoni svolgono la professione di giudici.

144 Vedi sopra, nota 75.

145 BRaIDI, I Modenesi nel Trecento, p. 146: Tommasino del Ferro è censito nel Liber magna massa Populi, compilato subito dopo la rivoluzione a guida popolare del gennaio 1306, accanto ai più stretti famigliari di Niccolò, residente come loro nel quartiere di porta San Pietro e censito nell'ambito della medesima societas Castellarii, il raggruppamento a base rionale collegato alla strada (odierna via Castellaro) ove si concentrano le case dei Mattarelli. Viene anche eletto, al fianco di Niccolò, nel Consiglio dei 400 e in quello dei 600, i due vasti organi consigliari formati nel 1306 e nel 1307 come esito delle incerte istituzioni di matrice popolare (ibidem, pp. 203 e 233).

146 Tommasino del Ferro, assieme ad Alessandro Mattarelli, fratello di Niccolò, nel 1307 è chiamato a testimoniare a due atti che riguardano il giurista modenese: ASMo, Memoriale antico, a. 1307/1, n. 278 (14 agosto 1307): Guido di Giunta dei Molza riceve in dote da Giovannibuono Mattarelli, che agisce a nome del padre Niccolò, la somma di 300 lire modenesi a titolo di dote per Richelda, figlia dello stesso Niccolò, destinata in sposa a Gerardino Molza, figlio di Guido; si aggiunge la somma di 10 lire a titolo di donatio simplex; ASMo, Memoriale antico, a. 1307/1, n. 1044 (11 aprile 1307): frate Pietro praeceptor domus militie Templi de Mutina conferma la vendita di una casa in Modena fatta al doctor legum Niccolò Mattarelli da Tomasina del fu Modenese di Enrico Scudari (per cui vedi sopra, nota 97).

147 ASMo, Memoriale antico, a. 1309, n. 4125 (28 settembre 1309). Ricordato in Vicini, Di Niccolò Mattarelli, p. 17 s. 


\section{Ancora nelle file del Popolo}

Rientrato a Modena nel 1306, Mattarelli concede in sposa la figlia Bartolomea a Manfredino di Egidio Pio ${ }^{148}$, rampollo di una delle famiglie dell'aristocrazia ghibellina già allontanate da Modena dal 1264 e più avverse al monopolio signorile instaurato in città dai marchesi d'Este con Obizzo II (1247 ca.-1293) e poi con il primogenito di questi Azzo VIII $(\dagger 1308)^{149}$. Una scelta che si può valutare come effetto dell'ostilità del Maestro verso il governo esercitato in Modena per più di quindici anni da Azzo e dal padre e come conseguente esito del suo avvicinamento alla fazione aristocratica storicamente contrapposta ai marchesi estensi e ai loro alleati modenesi, avvicinamento cementato tramite un'unione famigliare che è documentata in modo significativo anche dopo la scomparsa del giurista, già avvenuta nel giugno $1314^{150}$.

La presenza a Modena di Mattarelli dal maggio 1306, che rappresenta una importante cesura nell'arco della lunga docenza a Padova avviata - con certezza documentaria - almeno dall'autunno $1295^{151}$, assume una rilevanza senz'altro particolare nel conte-

148 ASMo, Memoriale antico, a. 1306/1, n. 1253 (13 maggio 1306): Manfredino di Egidio Pio, in presenza e con il consenso del padre, riceve a titolo di dote da Niccolò Mattarelli la somma di 400 lire modenesi per avere in sposa una delle figlie di questi, Bartolomea; si aggiunge la somma di 20 lire a titolo di donatio simplex; fra i testimoni anche Alessandro Mattarelli, uno dei fratelli di Niccolò.

149 Una buona sintesi dell'azione politica e del potere signorile esercitato dai due marchesi estensi in CHIAPPINI, Gli Estensi, p. 59 ss. Sui Pio, il loro stretto coinvolgimento nelle contese politiche interne al comune modenese e l'avvio della loro signoria sul castrum e il territorio di Carpi, dislocato a nord di Modena in direzione di Mantova, si rinvia ai saggi recenti di BonacinI, Il governo del comune cittadino tra Due e Trecento, e di Andreolli, Dai 'Figli di Manfredo' ai Pio.

150 ASMo, Memoriale antico, a. 1314, n. 3504 (10 giugno 1314): testamento dettato in Modena da Francischus legum doctor filius quondam domini Nicholay de Matarelis legum doctoris in favore dei figli Antonio, Gian Novello, Ubertino e Bartolomeo; fra i testimoni sono presenti anche Manfredino Pio e Gerardino Molza (generi di Niccolò e cognati di Francesco), Bartolomeo Spetiali (già presente all'atto di costituzione di dote in favore di Bartolomea, figlia di Niccolò, datato 13 maggio 1306) e il magistro Athaleo de Matharelis (nipote di Niccolò in quanto figlio del fratello Gian Novello).

151 Gloria, Monumenti della Università di Padova, p. 49 (5 novembre 1295): conferimento del dottorato in diritto canonico, da parte di Giovannibuono, vicario del Capitolo della chiesa di Padova, a Tommaso de' Lamandini, priore di S. Anto- 
sto dell'esperienza politica del giurista e del ruolo da lui giocato nell'ambito delle istituzioni cittadine; una nuova fase che acquista un significato anche maggiore rispetto a quella esauritasi nel corso degli anni Settanta del Duecento.

Il 26 gennaio 1306, infatti, una rivoluzione di matrice popolare, anticipata e sostenuta da diverse iniziative politico-militari concertate dalle forze ostili al dominio marchionale ramificate in altre città padane, scalza il potere estense e istituisce un nuovo governo che tempestivamente si organizza attraverso organi e magistrature nuove e si orienta a definire con precisione quanti appartengono al Populus, e quindi sono ammessi a ricoprire cariche rappresentative all'interno delle nuove istituzioni, e quanti sono invece riconosciuti nobiles et potentes, con conseguente esclusione dalla partecipazione alla vita politica cittadina.

Il nuovo assetto di quest'ultima non appare tuttavia orientato a segnare una distinzione netta e una linea invalicabile tra le due partes annullando completamente la capacità politica di potentes $\mathrm{e}$ magnati, ma unicamente a mitigarne l'accesso agli organi consiliari limitandola a soggetti e famiglie alleate della pars populi. Al fine di attuare tale riconoscimento, presupposto ora indispensabile per consentire la partecipazione politica, viene prontamente allestito un registro destinato a censire la Magna Massa Populi separatamente da quello delle famiglie e dei singoli individui riconosciuti invece come appartenenti alla pars nobilium ${ }^{152}$. Le liste così approntate non

nio in Bologna, presentato da Bovetino decretorum doctor; sono presenti quali doctores legum Niccolò Mattarelli, Riccardo Malombra e Giovanni de Rechenati regentes Padue, e come doctores Decretorum Bovetino canonico padovano, Taddeo de Pocaterris da Cesena e Altigrado da Lendinara regentes Padue. Il documento è ricordato in Colle, Storia scientifico-letteraria dello Studio di Padova, p. 78. Sul canonista Bovetino, oltre alla voce biografica anonima in DBI, 13, Roma 1971, pp. 543-546, si veda il più recente e ampio studio monografico di GARDONI, Per la biografia di Bovetino da Mantova decretorum doctor, integrato dalle più recenti indagini di ZacCaria, Nuovi contributi. Mattarelli, attestato a Padova nel luglio 1290, è già rientrato a Modena nel 1293: vedi sopra, nota 138 e cap. II, nota 7.

152 ASCMo, Camera segreta, III.1. Magna Massa Populi Civitatis Mutine, contenente ai ff. 1r-6v l'elenco degli esponenti della nobiltà cittadina suddivisi per gruppi familiari, con i nomi di 271 individui (pubblicato in VICINI, Il «Liber nobilium et potentum», pp. 172-188, e in BRAIDI, I Modenesi nel Trecento, pp. 95-109, con commento a p. $58 \mathrm{ss}$. Il numero dei nobiles risulta tuttavia molto più elevato, poiché in numerosi casi le famiglie sono citate in forma cumulativa oppure l'elenco degli appar- 
riflettono una rigida e impermeabile contrapposizione fra i due raggruppamenti, giacché vengono accettate forme di travaso reciproco per riconoscere pubblicamente i soggetti qui digni non sunt esse in populo presenti, che in alcuni casi ottengono pure di essere riammessi tra i populares, e nel medesimo tempo individuare nobili e magnati che per le loro esplicite scelte di campo sono subito accolti nelle file della parte popolare.

In essa, fin dalla redazione originaria della lunga lista che deve comprovare l'appartenenza al Populus di oltre 5.000 cittadini modenesi adulti, sono annoverati tanto Niccolò, unico doctor legum così registrato nella lista dei popolari, quanto i suoi più stretti famigliari: i figli Francesco e Giovannibuono, Antonio e Gian Novello, figli di Francesco, il fratello Alessandro con il figlio Corradino e quindi Diocles e Athaleus, figli dell'altro fratello Gian Novello ${ }^{153}$. Tutti risiedono nel quartiere di porta San Pietro e, all'interno di questo, appartengono alla Societas Castellarii, così denominata dalla strada che sbocca sulla piazza centrale della città ove - come già evidenziato - nei decenni precedenti si è concentrata la presenza del gruppo famigliare e la parte preponderante delle sue proprietà urbane.

La competenza tecnica e il prestigio di un doctor iuris già partecipe negli anni precedenti al funzionamento delle istituzioni cittadine costituiscono probabilmente validi motivi per eleggere anche Niccolò Mattarelli all'interno dei nuovi organi consiliari cui è affidato il governo della città. Nei primi giorni del febbraio 1306 egli viene eletto, assieme al fratello Alessandro, sia nel Consiglio dei 400, fra i 100 consiglieri chiamati a rappresentare il quartiere di porta $S$. Pietro, sia nel più ristretto organo formato da 40 sapientes, 10 per ciascuna delle quattro porte cittadine e sempre in rappresentanza

tenenti ad esse è seguito dalla specificazione et omnes alii de dicta domo, tam clerici quam layci et tam naturales quam legitimi), e ai ff. 14r-59r l'elenco assai più consistente degli appartenenti al populus (5.189 nomi), suddivisi in base alle societates a base rionale costituite sullo schema delle vecchie cinquantine duecentesche (pubblicato in EAD., I Modenesi nel Trecento, pp. 129-200, con commento a p. 62 ss.). I principi normativi che ispirarono la compilazione dei due elenchi sono espressi in RM I, p. 41. Sull'evoluzione delle condizioni politiche che portarono alla rivoluzione del gennaio 1306 si veda in particolare VICINI, La caduta del primo dominio estense, e, da ultimo, Braidi-Tavilla, Statuti del Collegio dei giudici e degli avvocati, p. 50 ss.

153 BraIDI, I Modenesi nel Trecento, p. 147 s. 
del quartiere di porta San Pietro, deputati ad providendum et ordinandum omne id et quicquid eisdem videretur circa reformationem et conservationem et bonum statum comunis et populli Mutine ${ }^{154}$.

La presenza del giurista risulta preziosa nel contesto delle giovani e incerte istituzioni che assumono frettolosamente il governo della città e del suo territorio, dovendo anche preoccuparsi della difesa militare di quest'ultimo a fronte di attacchi e interventi delle milizie guidate da Azzo, dai suoi famigliari e dalle famiglie alleate. Di conseguenza Mattarelli viene pure eletto tra i 16 Difensori della Libertà, organo esecutivo posto a fianco del Capitano del Popolo che riesuma quelli analoghi già sperimentati nelle fasi di partecipazione al governo cittadino da parte del Populus, in variabile condominio con le fazioni nobiliari, tra il 1249 e il 1264 e ancora tra il 1271 e il 1289. Si tratta, per l'esperto giurista, di un'esperienza politica già collaudata, dal momento che nel 1279 aveva già fatto parte della stessa magistratura, al tempo composta da 24 membri $^{155}$. E per dargli la possibilità di completare il nuovo mandato, due mesi più tardi il Consiglio del Popolo propone di inviare un ambasciatore a Padova per supplicare il Comune e la locale Universitas scholarium di concedere al professor legum modenese il permesso di trattenersi ancora nella propria città natale per totum mensem aprilis ${ }^{156}$.

154 Ibidem, p. 233 per la composizione del Consiglio dei 400, i risultati della cui elezione sono pubblicati il 5 febbraio 1306; il nome di Niccolò Mattarelli appare registrato per primo nella lista dei consiglieri eletti per porta San Pietro. RM I, p. 16 ( 8 febbraio 1306): il consiglio dei 40 sapientes, eletto su mandato conferito dal Consiglio generale al Capitano del Popolo e da questi eseguito tramite l'intervento dei frati Minori, dei Predicatori e degli Eremitani, dovrà poi sottoporre le proprie deliberazioni al Consiglio generale del Popolo. Alessandro de Matarello l'8 marzo 1307 appare anche tra i 4 sapientes eletti per la porta di S. Pietro (assieme ad altrettanti per ciascuno degli altri tre quartieri urbani) deputati a verificare e distribuire le provvigioni destinate ai capitani e alle guarnigioni dei castelli e dei fortilizi del Comune sparsi nel contado (RM II, p. 249).

155 Vedi sopra, nota 127.

156 AIMAe III, col. 908, trascritto anche in TIRABOschi, Biblioteca Modenese, III, p. 187 e in RM I, p. 117 (nell'ambito delle deliberazioni di vario contenuto assunte dal Consiglio del Popolo il 9 aprile 1306, alle pp. 115-119): «Item, si placet dicto Consilio quod unus ambaxator expensis Comunis et ad salarium contentum in statutis Comunis, mittatur ad civitatem Padue ad requirendum et precandum Comune Padue et Universitatem Scholarium, et prout aliter fuerit necessarium ex parte Comunis Mutine, quod eis placeat, precibus et amore Comunis Mutine, dare et concedere licentiam sapienti viro domino Nicolao de Mattarellis professori le- 
Nella tumultuosa fase politica conseguente alla caduta degli Estensi i legami con Padova e con il suo ceto dominate risultano comunque solidi: accanto al rientro in patria del giurista modenese si segnala pure la chiamata come podestà del giudice Enselmino degli Enselmini, dotato di specifica preparazione tecnica e vicino a una fazione «politicamente non ortodossa» ma riconosciuta come ghibellina in ambito padovano ${ }^{157}$.

In realtà il soggiorno modenese di Mattarelli si prolunga ancora a lungo, benché non si escluda che possa venire interrotto da saltuari viaggi a Padova. Ancora nel 1307 egli è a Modena, eletto tra $i$ 600 componenti il Consiglio del Popolo sempre in rappresentanza del quartiere di porta San Pietro, ed è ancora registrato al primo posto nell'elenco dei consiglieri eletti per il quartiere di porta San Pietro ${ }^{158}$. Ma è l'ultimo contributo alla politica cittadina che può offrire l'esperto ma ormai anziano giurista.

La rivoluzione della parte popolare non riesce a garantire la pace all'interno del comune e non riesce a evitare la ripresa violenta degli scontri entro lo spazio urbano fra le fazioni nobiliari degli Aigoni di orientamento guelfo, guidati dalle famiglie Rangoni, Boschetti, Guidoni e da Savignano, e dei Grasolfi filoimperiali, polarizzati attorno ai da Sassuolo e ai Grassoni. La lotta armata si estende ad alcune roccaforti militari del contado e coinvolge il vicino comune di Bologna, che nel 1308, una volta scomparso il marchese Azzo VIII d'Este, interviene con successo al fianco degli Aigoni già esuli dalla città sconfiggendo l'esercito modenese ${ }^{159}$.

gum, standi Mutine per totum mensem aprilis ad complendum officium Defensoris libertatis Populi Mutine, in quo fuit electus, et quod iurare et exercere compulsus fuit per dominum capitaneum Populi Mutine, cum presentia dicti domini Nicolai dicto Populo et Comuni utilis et necessaria censeatur, et quod massarius generalis Comunis possit et teneatur facere solutionem dicto ambaxatori de avere Comunis libere et impune». La richiesta inoltrata alle autorità padovane, ricordata anche in Bortolami, Politica e cultura, p. 245, avrebbe avuto lo scopo di fare rientrare temporaneamente a Modena Mattarelli «per fungere da Difensore della libertà di quel popolo».

157 Ibidem, p. 255.

158 BRAIDI, I Modenesi nel Trecento, p. 203.

159 Una sintesi di queste convulse vicende politico-militari che vanificano rapidamente il progetto politico popolare avviato dagli inizi del 1306 in BraIDI, I Modenesi nel Trecento, p. 75 ss. 
Nel clima di crescente instabilità che pervade la società e la politica cittadina segnando il sostanziale fallimento delle prospettive inaugurate con la rivoluzione popolare del gennaio 1306 Niccolò Mattarelli pare defilarsi e già non risulta più eletto nel Consiglio dei 400 rinnovato nel $1308^{160}$. Ciò pone termine in via definitiva al suo diretto coinvolgimento negli organi istituzionali del comune modenese che aveva segnato due fasi importanti della sua vita, una prima volta nel corso degli anni Settanta del Duecento e in seguito, superati ormai i sessant'anni di età, in occasione della più originale esperienza politica conseguente all'abbattimento della prima signoria estense sulla città, perdurata fra il 1289 e i primissimi giorni del 1306.

\section{Il ritorno a Padova}

Esaurita la partecipazione diretta agli organi rappresentativi della Respublica mutinensis, dal maggio 1308 Mattarelli riprende l'attività presso lo Studio di Padova ${ }^{161}$ raggiungendo nuovamente il figlio Francesco, come il padre docente di diritto civile e in tali funzioni documentato dal febbraio $1305^{162}$ sino al luglio $1310^{163}$. In quella sede Mattarelli insegnava almeno dall'autunno del $1295^{164} \mathrm{e}$

160 Ibidem, p. 219 ss.

161 Vedi sotto, cap. II, nota 9.

162 Marangon, Gli «Studia» degli ordini mendicanti, n. 2, p. 101 (12 febbraio 1305): nel Maggior Consiglio del Comune di Padova, di fronte anche al podestà Pace de Pacibus de Bononia legum doctor, viene letto il consilium formulato dal legum doctor Pace de Tadis, dal giudice Paolo de Titulo e da dieci doctores salariati sia di diritto civile che canonico, ove si riconosce il diritto del Comune alla terza parte della condanna inflitta dall'ufficio dell'Inquisizione a Ezzelino da Este; fra i dieci doctores vi sono Niccolò Mattarelli con il figlio Francesco, Riccardo Malombra e Oldrado da Lodi.

163 Gloria, Monumenti della Università di Padova, p. 71 (25 luglio 1310). Breve profilo di Francesco Mattarelli in ID., Monumenti della Università di Padova, p. 260 s. e in Vicini, Di Niccolò Mattarelli, p. 27 s., ove tuttavia si data al 1308 la prima documentazione del suo insegnamento civilistico a Padova.

164 Vedi sopra nota 151. Mattarelli è già presente a Padova nel luglio 1290 in veste di testimone a un atto stipulato tra un privato e i ministri dei frati di penitenza: vedi sotto, cap. II, nota 7. 
nel dicembre 1305 disputa una quaestio in materia di dote conservata in due noti manoscritti vaticani ${ }^{165}$. In data imprecisata affronta anche una quaestio [quae] fuit Padue de facto, utilizzata già da altri giuristi quali Federico delle Scale, Francesco d'Accursio e Dino del Mugello, inerente l'azione intrapresa dal Comune per obbligare alla corresponsione di honera et factiones dicti communis un soggetto che invece si opponeva adducendo la ragione che neppure il padre mai li aveva sostenuti ${ }^{166}$. Sempre nella città veneta rilascia un consiglio in merito alla pena da comminare a chi smercia monete false affiancando il proprio parere a quello espresso da altri legum doctores legentes Padue, ossia Giovanni d'Anguissola, che vi insegna dal 1275, Iacopo d'Arena, Bartolus de Corazino e Federico delle Scale, documentato a Padova nel $1284^{167}$.

Fin dal dicembre 1281, poco prima di lasciare Modena per as-

165 Quaestio «Statutum est in civitate quod filia familias decedente matrimonio liberis extantibus» trádita in ASP A.29, ff. 288rb-289vb e in Chigi E.VIII.245, f. 129ra-vb. Si veda Bellomo, I fatti e il diritto, p. 189 e ID., Quaestiones in iure civili disputate, n. 363, p. 187 e n. 182, p. 292 s. Si ricorda anche una quaestio de facto in materia di sostituzione pupillare che fuit in civitate Mutine, ma che Mattarelli potrebbe avere riproposto nel corso della sua lunga permanenza a Padova: «Quidam filios non habens fecit testamentum in quo talem fecit institutionem et substitutionem»: in ASP A.29, ff. 276ra-277va e in Chigi E.VIII.245, ff. 140va-141rb, per cui si veda sotto, cap. II, nota 92.

166 Chigi, E.VIII.245, ff. 76rb-78rb: «Hec quaestio fuit Padue de facto. Communis consilium agebat contra quendam et dicebat eum teneri subire honera et factiones dicti communis [...]»; si veda Bellomo, Quaestiones in iure civili disputatae, n. 92, p. 254. Nel testo viene citato il giudice Obizzo, assessore del podestà di Padova Barone, nel quale si può riconoscere Barone dei Mangiatori da San Miniato, alla guida del comune padovano nel 1286: Annales Patavini, pp. 205, 230; Liber Regiminum Padue, p. 338 s. A tale quaestio de facto fa riferimento Baldo in BALDI UbaLDI, In sextum codicis librum commentaria, f. 26ra, n. 8 (ad C. 6.7.2).

167 «Quesitum fuit Padue qua pena puniatur ille qui expendit falsam monetam scienter» (Collegio di Spagna 126, f. 228v); si veda MAFFEI, I codici del Collegio di Spagna, p. 437. In calce al testo, in colonna sul lato sinistro del foglio, sono riportati i nomi di Ioannes de A(n)guisellis, Iacobus de Aretio, Bartolus de Corazino, Federicus de Lascha, Nicolaus de Matharelli, tutti assieme qualificati come legum doctores legentes Paduae. Assecondando l'orientamento del podestà, secondo il quale falsarios expendentes falsam monetam comburi (debeant), e sulla base del combinato disposto di D. 3.2.13.7 (De his qui notantur infamia) e C. 9.24.2 (De falsa moneta), l'opinione dei doctores interrogati sul punto risulta pienamente concorde (et sic volumus de facto fore pronuntiandum). Circa Giovanni d'Anguissola, originario di Cesena, si rinvia al breve profilo di Mellusi, Giovanni d'Anguissola. Il consilium è ricordato da EmiLı, Fridericus de Scalis, p. 583, n. 2. 
sumere l'incarico annuale di consultore a Foligno, Mattarelli aveva dettato le ultime volontà istituendo eredi i tre figli maschi Francesco, Giovannibuono e Manfredino e disponendo in favore dei figli e delle figlie che essi avrebbero avuto la somma non modesta di 200 lire modenesi ${ }^{168}$. Quanto alle figlie femmine, Bartolomea e Richelda, nel periodo in cui egli rientra a Modena tra 1306 e 1307 provvede ad accasarle dandole in moglie ai rampolli di due note famiglie dell'aristocrazia cittadina: la prima a Manfredino di Egidio Pio con una dote di 400 lire modenesi e la seconda a Gerardino di Guido Molza con una dote di 300 lire $^{169}$.

Alla data del 13 maggio 1306 Egidio Pio e i figli erano già stati verosimilmente liberati dal carcere in cui erano stati trattenuti in via temporanea allo scoppio improvviso della rivoluzione popolare nel precedente mese di gennaio ${ }^{170}$, mentre l'atto di costituzione di dote in favore di Richelda viene disposto il 14 agosto 1307 non direttamente da Niccolò, ma dal figlio Giovannibuono, forse perché il padre, eletto in quell'anno nel Consiglio dei 600 del Popolo, aveva già lasciato la città nel corso dell'estate o poco prima per rientrare a Padova.

Negli ultimi anni di attività di Mattarelli si può collocare la discussione relativa alla successione nel regno di Napoli, che assume un certo rilievo già al momento dell'investitura a duca di Calabria di Roberto d'Angiò il 2 febbraio 1297, accompagnata dalla sua designazione a erede al trono, e acquista ancor maggiore risonanza alla morte del padre Carlo II, avvenuta il 5 maggio 1309171; questione che egli affronta nella parte finale del commento al titolo del Digesto de iurisdictione omnium iudicum (D. 2.1), comunque a noi non

168 ASMo, Memoriale antico, a. 1281, n. 2351 (17 dicembre 1281): Niccolò Mattarelli detta il proprio testamento istituendo eredi i figli Francesco, Giovannibuono e Manfredino et postumos mascullos qui sibi nascerentur et si postuma unam vel plures nascerentur unam quamcumque in ducentarum libras mutine sibi haeredem instituit. Allo stato delle fonti conosciamo soltanto i nomi di quattro figli maschi del solo Francesco e dei figli di due soli di essi, Ubertino e Bartolomeo.

169 ASMo, Memoriale antico, a. 1306/1, n. 1253 (13 maggio 1306) e a. 1307/1, n. 278: vedi sopra note 146 e 148 .

170 Come documentato nel Liber nobilium et potentum redatto nel febbraio 1306: BRAIDI, I Modenesi nel Trecento, p. 106.

171 Per tali dinamiche successorie basti il rinvio a Galasso, Il Regno di Napoli, p. 114 ss.; Tramontana, Il Mezzogiorno medievale, p. 138 ss. 
pervenuto. Come riferisce molti anni dopo Baldo, del tema si erano occupati Riccardo Malombra e dopo di lui Cino e Bartolo risolvendolo pacificamente in favore di Roberto d'Angiò (1277-1343), terzo figlio di Carlo sopravvissuto al primogenito Carlo Martello (12711295) e al fratello Ludovico (1274-1297), entrato nell'ordine francescano $^{172}$. Diversamente Mattarelli aliter decidit preferendo riconoscere il diritto di successione al figlio del defunto Carlo Martello, Carlo Roberto (1288-1342), con un ragionamento più approfondito che discende dall'iniziale distinzione in merito all'intentio concedentis, nei casi in cui essa proceda ex verbis, vel ex consuetudine regionis vel ex consuetudine cedentis, et tunc servabitur quod actum est. Non potendo tuttavia determinarla su queste basi, e dichiarando che haec iurisdictio est feudum et iure feudi concessa, allora quest'ultima può transitare al nipote quia sic est in quolibet feudo ut nepos succedat avo in locum sui patris; se invece non erat feudo data, tunc magis ad filium debet pertinere quam ad nepotem, in quanto si configura non iure successionis, sive hereditatis e si trasmette ex proximitate liberorum.

Nel primo caso, preferito da Mattarelli, la trasmissione del potere regio che si materializza nell'alta funzione della iurisdictio si configura come delega da concedersi tramite gli strumenti del diritto feudale e l'erede diretto del primogenito conserva quindi il diritto a subentrare al padre già defunto, mentre nel secondo essa è declinata sotto forma patrimoniale e il diritto all'eredità segue la linea di successione in favore dei discendenti diretti del sovrano scomparso. Per quanto la prima fattispecie trovi corrispondenza nell'effettivo assetto giuridico-politico del grande regno del Mezzogiorno, le cui radici legittimanti affondavano nel concordato stipulato da papa $\mathrm{Ni}$ colò II con Roberto il Guiscardo in occasione del concilio di Melfi

172 Baldi UbALDI, In sextum codicis librum commentaria, f. 22ra-b, n. 24 (ad C. 6.3.10[6]), da cui le citazioni utilizzate di seguito nel testo. La quaestio, agitata anche nello studio padovano, ove Mattarelli e Malombra furono colleghi sino al 1310-11, è sinteticamente ricordata da BESTA, Riccardo Malombra, p. 131 s. La posizione di Riccardo Malombra, favorevole alla successione del secondogenito Roberto sulla base del principio per cui filii esse deteriores nepotibus non sinantur, è ricordata in CinO DA PISTOIA, Lectura super Codice, II, f. 434ra (ad C. 6.59[60].3) e da qui ripresa da Giovanni d'Andrea nell'ambito di una lunga additio a GugLIELmo Durante, Speculum iudiciale, IV, III, De feudis, in rubrica, t. 2, p. 311. 
nell'agosto $1059^{173}$, l'approccio teorico di Mattarelli non condiziona l'incoronazione a re di Napoli di Roberto d'Angiò, disposta ad Avignone da papa Clemente V il 3 agosto 1309, e la successiva azione dello stesso Roberto quale vertice della coalizione guelfa ostile al programma di ricostruzione del potere imperiale in Italia tentato da Enrico VII.

Ancora nel luglio 1310 Niccolò Mattarelli figura tra i docenti salariati dello Studio padovano ${ }^{174}$, ma risulta già defunto quattro anni dopo, nel momento in cui il figlio Francesco detta in Modena il proprio testamento ${ }^{175}$. Risultano parimenti incerte le sorti della sua sepoltura, che iniziano subito a intrecciarsi con quelle della sua memoria presso i posteri e della fortuna delle sue opere trovando una sponda importante nel contesto della sua città di origine, anche se soltanto a due secoli di distanza dalla scomparsa.

Bernardino Scodobi (1527-1550) è giurista assai precoce, di natali modenesi e allievo per quattro anni a Bologna di Andrea Alciato; di lui ci è giunta una interpretatio della costituzione Habita data alle stampe in Venezia nel 1547-48, pochi anni prima della morte del giovanissimo autore ${ }^{176}$. L'opera, introdotta da un indirizzo di saluto

173 Il quale, giurando fedeltà al pontefice, viene riconosciuto «duca di Puglia e di Calabria per grazia di Dio e di San Pietro e, con l'aiuto di entrambi, futuro duca di Sicilia»: Tramontana, La monarchia normanna e sveva, p. 496 ss.; ID., Il Mezzogiorno medievale, p. 22 s.; Houben, I Normanni, p. 65, da cui la citazione.

174 Gloria, Monumenti della Università di Padova, p. 71, 1310 luglio 25 (ricordato anche a p. 249, nota 7, e p. 387): conferimento del dottorato in diritto civile, nella cattedrale di Padova, a Giovanni di Giroldo da Losanna, presentato da Niccolò Mattarelli cum aliis doctoribus, ossia Riccardo Malombra, Oldrado da Ponte, Francesco Mattarelli, figlio dello stesso Niccolò, il bolognese Paolo de Sulimano e i canonisti Egidiolo da Cremona e Guido Ferri da Mantova. Vedi pure VicinI, Di Niccolò Mattarelli, p. 20. Pochi mesi prima uno dei figli di Niccolò, Giovannibuono, emancipato dal padre, aveva acquistato un terreno di 4 biolche: ASMo, Memoriale antico, a. 1310/1, n. 1900 (21 aprile 1310).

175 ASMo, Memoriale antico, a. 1314, n. 3504, 1314 giugno 10, lunedì: testamento dettato in Modena da Francischus legum doctor filius quondam domini $\mathrm{Ni}$ cholay de Matarelis legum doctoris in favore dei figli Antonio, Gian Novello, Ubertino e Bartolomeo.

176 Bernardini ScodobiI, In auctenticam habita; cfr. f. $41 \mathrm{v}$ per la conferma dell'origine modenese di Scodobi («Quemadmodum Mutinae patrie mee dulcissime evenisse legitur»), analogamente a Mattarelli (f. 54r: «et Nicolaus Matterellus civis noster ait»). Si veda pure a f. 40v per il grato ricordo dell’Alciato («ubi [scil. Bononiae] ego quoque cum plusquam sesqui mille scholasticis, sub magno illo iurecon- 
al lettore composto da Francesco Bellincini (1495-1565), giureconsulto sempre modenese intenzionato a celebrare in chiave squisitamente encomiastica le qualità umane e la sapienza dottrinaria del promettente Scodobi ${ }^{177}$, viene corredata da fitte aggiunte marginali che è verosimile attribuire al medesimo Bellincini sia per la comune origine cittadina, sia per la conoscenza che poteva legarlo al più giovane Scodobi, da lui segnalato e valorizzato agli occhi del benignus lector tramite la composizione del testo iniziale.

Ed è una nota a margine della pagina, ove si ricorda Nicolaus Matterellus item celebratissimus in eisdem scholis professor et civis, a riportare la precisa indicazione del luogo del suo sepolcro, collocato nel sotterraneo della duecentesca chiesa dei Predicatori di Mo-

sultorum principe et doctrinae legalis thesaurario Andrea Alciato annis quatuor militavi») e anche a f. 101v, ove è ribadito un ampio panegirico del giurista milanese che conferma la devozione dell'allievo nei confronti del maestro, oltre a numerosissime e costanti citazioni dalle sue opere distribuite in tutta l'opera. Alciato insegna effettivamente a Bologna dal 1537 al 1541 e perciò si deve ammettere che Scodobi abbia avviato gli studi giuridici in assai giovane età. Scarne notizie biografiche su di lui, per gran parte desunte dai riferimenti sparsi nella sua stessa opera, si devono a Vedriani, Dottori modonesi, p. 134 s.; Tiraboschi, Biblioteca modenese, V, pp. 185-190 (ed. 1784); ID., Storia della letteratura italiana, V/1, pp. 61-84 e V/2, pp. 430-434. L'opera di Scodobi è censita in ZiLETTI, Index librorum omnium, f. 20r, tra le repetitiones alla costituzione Habita inserita nel titolo Ne filius pro patre (C. 4.13). Fontana, Amphiteatrum legale, col. 39, attribuisce a Scodobi non meglio specificati «commentaria in $\mathrm{l}$. Si [ex] cautione de non numer. pecunia» (C. 4.30.3), rinviando all'indice di Ziletti, ove invece in relazione a questo titolo del Codice è ricordata soltanto una repetitio alla 1. generaliter (C. 4.30.13) attribuita a Pierre de Belleperche (ZILETTI, Index librorum omnium, f. 20r).

177 Sul Bellincini si vedano i profili biografici di Vedriani, Dottori modonesi, pp. 72-74; Forciroli, Vite dei modenesi illustri, p. 122 s.; TiRABOschi, Storia della letteratura italiana, III, p. 593 s. Studia diritto a Ferrara e poi svolge una prestigiosa carriera che lo conduce al servizio di alcune tra le maggiori corti rinascimentali italiane: svolge l'ufficio di podestà a Parma nel 1528-29, è auditore della Rota di Genova e giudice a Urbino; chiamato da papa Paolo III a Roma, gli viene conferita la dignità di senatore e l'incarico di amministrare la giustizia; in seguito si trasferisce a Ferrara, invitato dal duca Ercole III con il titolo di consigliere e segretario di giustizia. Nella stessa famiglia si ricorda pure la figura del giurista Bartolomeo (1428-1478), laureatosi a Ferrara, docente di diritto canonico nella capitale estense e poi a Bologna e infine chiamato da Sisto IV a Roma come uditore di Rota: a lui sono dedicati i sintetici profili biografici di Prosdocimi, Bellencini Bartolomeo, e di TAVILla, Bellencini (Bellincini) Bartolomeo. Opere manoscritte di Bartolomeo Bellincini di ambito canonistico sono presenti nella Biblioteca di Felino Sandei (14441503 ) e da questi ampiamente utilizzate: Pomaro, I manoscritti medievali della Biblioteca capitolare feliniana, pp. 165, 188, 213. 
dena ${ }^{178}$. Notizia che pare attendibile, giacché proviene dal medesimo ambiente urbano e professionale di Mattarelli, anche se oggi non più verificabile a causa delle profonde modificazioni subite dalla chiesa di San Domenico, ampliata e trasformata già tra la fine del secolo XIV e la metà del successivo e quindi rimodellata completamente nei primi decenni del Settecento per armonizzarne i volumi e l'orientamento rispetto al vicino palazzo ducale estense ${ }^{179}$.

Diversamente, una notizia riferita per la prima volta da Guido Panciroli (1523-1599) - e pertanto cronologicamente vicina all'esperienza personale e alle opere di Scodobi e Bellincini - vorrebbe che Mattarelli sullo scorcio della propria vita avesse esercitato l'ufficio di giudice a Lucca e in quella città fosse morto ${ }^{180}$, aprendo così ulteriori interrogativi sulla presenza nella città toscana di esemplari manoscritti delle sue opere, che egli avrebbe recato con sé e lì avrebbe abbandonato causa l'improvvisa scomparsa ${ }^{181}$. Ma a fronte dell'impossibilità di rintracciare testimonianze in grado di fornire indicazioni certe sulla morte del giurista modenese e sul luogo della sua sepoltura ${ }^{182}$, il filo di quelle relative invece alla sua produzione dottrinaria può essere sviluppato grazie a dati originali che consentono di integrare in misura apprezzabile le conoscenze acquisite sino a oggi.

178 Bernardini Scodobil, In auctenticam habita, f. 42r.

179 Soli, La chiesa di S. Matteo, poi di S. Domenico, pp. 354 ss., 389 ss.

180 Guidi Panziroli, De claris legum interpretibus, p. 181 (ed. Lipsiae 1721, p. 146): «Ferunt eum [scil.: Mattarelli] postremo praetorem Lucae ius dicentem in magistratu decessisse».

181 Per tali problematiche si rinvia al cap. III.

182 L'esistenza di una «statua» di Mattarelli all'interno del Duomo di Modena presso l'altare della famiglia Sadoleto è ricordata da Ludovico Vedriani nel tardo Seicento (cfr. cap. III, nota 105), ma non se ne trova alcuna menzione nelle memorie relative all'altare e alla cappella che lo custodiva. La cappella, intitolata alla SS. Trinità e promossa dal consigliere ducale Nicolò Sadoleto tramite un legato disposto con atto del 18 agosto 1495, venne ceduta dall'ultimo esponente della famiglia, il canonico Jacopo Sadoleto, nel dicembre 1646 al vescovo Roberto Fontana, che in seguito ne curò l'ampliamento facendovi allestire il proprio monumento funebre, già terminato nel 1652, nel quale venne tumulato nel 1654: Dond, Notizie storiche ed artistiche del Duomo di Modena, p. 257 s.; Baracchi-Giovannini, Il Duomo e la torre di Modena, p. 247 ss.; Frugoni, Il Duomo di Modena. Testi, p. 270 s. 



\section{CAPitolo II}

\section{FONS BONA, NIHIL ALIUD \\ OPERE DOTTRINARIE E LORO RICEZIONE NELLA SCIENZA GIURIDICA}

Sommario: 1. Nessuno è profeta in patria - 2. Accipe igitur librum sacratissimarum legum -3 . Tracce sparse di attività didattica -4 . Repetitiones e tractatus - 5. Nello strumentario del giurista postaccursiano: le quaestiones - 6. Libri magni quaestionum - 7. Un isolato interesse canonistico - 8. De hac materia summam prolixam formavit -8.1. Le imbreviature dei notai-8.2. Le scritture dei mercanti.

\section{Nessuno è profeta in patria}

A Modena, patria di Mattarelli, la consapevolezza di potere disporre in misura assai limitata delle opere dell'illustre conterraneo, scomparso tra il luglio 1310 e il giugno $1314^{1}$, emerge già nel tardo Trecento $^{2}$ e trova conferma due secoli più tardi, quando esse -

1 Vedi sopra, cap. I, note 174 e 175.

2 Vischi, Sandonnini, Raselli, Cronache modenesi, p. 206 (cronaca Tassoni, che riproduce gli Annales Veteres Mutinenses leggibili nella più risalente e meno affidabile trascrizione in RIS I, vol. XI, coll. 53-86, a col. 81): in riferimento all'anno 1334 , di seguito a varie notizie relative ai movimenti militari che interessano il territorio modenese coinvolgendo sia potenze esterne come Scaligeri, Gonzaga e marchesi estensi, sia gli intrinseci modenesi nella lotta contro altre famiglie dell'aristocrazia locale, si ricorda che «Hoc tempore floruit Nicolaus Mattarelus jurisconsultissimus mutinensis, qui composuit multa et maxime super Digestis et Codice, quamvis pauca reperiantur». Su caratteri e qualità di questi testi cronachistici si veda la scheda curata da Bruno Andreolli in Andreolli, GatTI, GreCI, Repertorio della cronachistica, p. 211 ss. 
$\mathrm{o}$, più verosimilmente, alcuni selezionati testimoni - sarebbero state individuate a Lucca, ove si riteneva che Mattarelli fosse spirato trovandosi a ricoprire la carica di giudice del podestà ${ }^{3}$. All'epoca, in ogni caso, il Consiglio della Comunità modenese non sembra avesse dato seguito alla proposta di farne eseguire copia a spese pubbliche ${ }^{4}$. E anche modernamente non se ne rinvengono esemplari all'interno di quella che spicca come il più rilevante deposito di codici manoscritti presente in città, ovvero la Biblioteca Feliniana di Lucca, il cui fondo principale non risulta tuttavia di formazione locale, ma è costituito dalla raccolta personale di Felino Sandei (14441503) lasciata in eredità al Capitolo del Duomo, incrementata dal fondo Capitolare aggiuntosi in tempi successivi e da materiali di più recente acquisizione ${ }^{5}$.

3 Come riferito pure da Guido Panciroli, il quale tramanda notizia che Mattarelli avesse ricoperto un incarico giudiziario a Lucca e che ivi sarebbe morto: vedi cap. I, nota 180 .

4 Cronaca modenese di Tommasino de' Bianchi, p. 143: [29 febbraio 1552] «Item el $\mathrm{M}^{\mathrm{co}} \mathrm{M}$. Lodovigo di Bianchi dottore che al presente è in la rota de Lucha ha scritto che in Luca ge le opere de M. Nicolò Matarello già dottore modenese valentissimo in lege ma che el bisognaria farle transcrivere in bona littera et s'è ottenuto el partito de farle transcrivere pensande de spendere pochi dinari»; p. 151: [11 marzo 1552] «Venerdì a di 11 ditto. Nui Conservatori se siamo adunati questa matina et è comparso M. Carolo Codebò dottore e fatto instantia che le opere del Matarello dottore antiquo modenese che se sono atrovate in Lucha per littere de M. Lodovico di Bianchi che è in la rota de detta città che le se dobiano fare transcrivere per poterle fare stampare, e la instantia che fa detti dottori voleno che la $\mathrm{M}^{\text {ca }}$ Comunità spenda per lori quello che doveriano spendere lori, perché li adoprano lori, la quale scriptura potria montare delli scuti centi. Et io Thomasino ge ho detto al capo di bancha M. Pietro Foschero che el se intenda quanto la montarà e non me ha voluto ascoltare». Sulla Rota o Consiglio di Giustizia attivo a Lucca dal $1530 \mathrm{si}$ veda Ascheri, Tribunali, giuristi e istituzioni, in part. p. 118.

5 Sintetico profilo biografico curato da MonTorzi, Sandei Felino, p. 1782 s. e anche ID., Felino Sandei, p. 275: il giurista ed ecclesiastico originario di Felina, nell'Appennino reggiano, è investito nell'agosto 1499 del vescovado di Lucca, ove tuttavia risiede pochissimo continuando le attività intraprese da oltre un decennio presso la Curia romana come uditore di Rota, membro della segreteria pontificia e della Segnatura apostolica. Altra bibliografia aggiornata nel recente approfondimento di alcuni profili del pensiero di Sandei messo a punto da di Renzo Villata, Diritto canonico, ricchezza e... povertà nell'opera di Felino Sandei. Per la formazione della Biblioteca Capitolare e i manoscritti ivi conservati si fa riferimento al recentissimo catalogo curato da PomAro, I manoscritti medievali della Biblioteca Capitolare, unitamente ai saggi introduttivi allo stesso, e a Murano, Felino Sandei (le opere e la biblioteca). Rilevanti manoscritti giuridici presenti nel fondo principale 
A conferma della debole e incerta fortuna che ormai segnava l'opera di Mattarelli nonché della fama più complessiva del giurista fissatasi nella memoria dei posteri, nei primi anni del Seicento il letterato modenese Girolamo Briani (1581-1646) porta proprio lui a singolare e paradigmatico esempio della sventura patita da molti «virtuosi» originari della medesima patria: un «famoso giurista de' tempi antichi», nel frontespizio dei cui libri campeggerebbe idealmente un motto non proprio lusinghiero per definirne la qualità scientifica e, assieme, la fama dell'autore: fons bona, nihil aliud ${ }^{6}$. A lui si attribuivano opere affidabili per i riferimenti che se ne potevano trarre e le allegazioni disperse nella posteriore letteratura giuridica, ma non certamente in grado di segnare tappe incisive nello sviluppo della dottrina e di perpetuare il nome dell'autore al livello di quello dei più celebrati maestri.

Si tratta - con tutta probabilità - di un giudizio di valore consolidatosi nella cultura municipale locale ed espresso tramite una metafora che prescinde dal riferimento a opere specifiche, ma tende piuttosto a qualificarne l'eredità giuridica in senso complessivo, almeno rispetto alla memoria tramandatasi in ambiente modenese, ove, in ogni caso, si era perduta la capacità di fornire elementi più certi e precisi in merito all'effettiva circolazione dei suoi scritti e alla loro conoscenza puntuale. Il progresso delle ricerche negli ultimi anni ha invece sovvertito in misura notevole tali valutazioni, consentendo di recuperare una serie di testi riconducibili a Mattarelli, sparsi in manoscritti distribuiti su scala europea, che appare rilevante sia per consistenza complessiva sia per varietà tipologica.

della Biblioteca sono oggetto di studio in SorrentI, Tra «lecturae»e «quaestiones»; MARTINo, «Lecturae per viam additionum»; ColLI, Il cod. 351 della Biblioteca capitolare «Feliniana» di Lucca.

6 Briani, Aggiunta a' Ragguagli di Parnaso, p. 19 s.: «Risguardo fra gli altri il premio che riportò Niccolò Matarello, famoso giurista de' tempi antichi, che troverai nel frontespicio de' suoi libri: fons bona, nihil aliud». In parte differente la versione riportata in Colle, Storia scientifico-letteraria dello Studio di Padova, p. 80: «Risguarda (dice) tra gli altri il premio che riportò Niccolò Mattarelli, famoso giurista dei tempi antichi, che troverai nel frontespizio de' suoi libri: Ars bona, nihil aliud». L'opera di Briani, pubblicata per la prima volta a Modena nel 1610, viene ristampata dal 1615 in poi variamente collocata di seguito ai Ragguagli del Boccalini: De CAro, Briani Girolamo, p. 212. 
2. Accipe igitur librum sacratissimarum legum

Non è dato sapere con esattezza in quale delle città ove opera come docente (Modena, Padova e probabilmente Cremona) oppure come giudice (Foligno) Mattarelli abbia composto i testi che a noi sono giunti sia in forma compiuta, dispersi nei fondi manoscritti di numerose biblioteche italiane e straniere, sia variamente ricordati in opere di diversi autori di età successive. Certamente un punto di riferimento importante nella sua produzione dottrinaria è rappresentato dalla città veneta, ove il giurista è presente già nel luglio $1290^{7}$ e poi con certezza nel ruolo di docente presso lo Studium dal novembre 1295 al luglio 1310. Anche le testimonianze fornite in merito da altri giuristi sottolineano il legame privilegiato tra la produzione scritta di Mattarelli e la sua presenza padovana.

Nell'arco dei molti anni in cui insegna in questa città Mattarelli partecipa in cattedrale alla solenne cerimonia di conferimento del titolo di dottore in diritto canonico oppure civile a studenti via via presentati dal doctor decretorum Bovetino da Mantova ${ }^{8}$, dai colleghi civilisti Riccardo Malombra e Oldrado da $\operatorname{Lodi}^{9}$ e da lui stes-

7 La prima attestazione del legum doctor Niccolò Mattarelli a Padova risale al 23 luglio 1290, giorno in cui egli testimonia, nell'ufficio dell'Inquisizione presso il convento di S. Antonio, assieme a Riccardo Malombra e al giudice Lovato del fu Rolandino da Lovato, a una transazione tra Leone de' Malcapelli e i ministri dei frati di penitenza (Gloria, Monumenti della Università di Padova, p. 43; atto ricordato anche a p. 249; BESTA, Riccardo Malombra, p. 11, nota 4; BONATO, Il «Liber contractuum» dei Frati Minori, n. 332, p. 789).

8 Vedi sopra cap. I, nota 165.

9 Gloria, Monumenti della Università di Padova, p. 65 s. (1308 maggio 25): conferimento del dottorato in diritto civile a Pietro Tome figlio di Guglielmo Tome de Hemanzo e a Bernardo de Codolis del fu Stefano de Codolis de Hemanzo, presentati da Riccardo Malombra e da Oldrado da Ponte da Lodi; sono presenti, accanto al rettore degli scolari ultramontani, Dadeo da Cesena, Giovanni d'Andrea da Bologna, Lichanore di S. Lucia da Padova, Guido a Ferris da Mantova, Egidiolo da Cremona decretorum doctoribus e Niccolò Mattarelli, legum doctore, omnibus sallariatis et actu regentibus in studio paduano, oltre a Giovanni de Ursinis canonico di S. Pietro de Urbe, Barnaba Malaspina, Tomasino de Cazadraghis da Mantova e Belcaro da Padova legum professoribus. Gloria, Monumenti della Università di Padova, p. 66 s. (1308 settembre 15, nella chiesa cattedrale di Padova): conferimento del dottorato in diritto civile a Francesco di Berto de Robertis da Borgo S. Sepolcro, presentato da Riccardo Malombra; sono presenti, accanto ai rettore degli scolari ultramontani e citramontani, Dadeo da Cesena, Giovanni d'Andrea da Bologna, Li- 
$\mathrm{so}^{10}$. In una occasione, nel 1308, è affiancato dal figlio Francesco, legum doctor, il quale insegna a Padova già dal $1305^{11}$, è ancora accanto al padre nell'aprile $1309^{12}$ e viene ricordato da Giovanni D’Andrea, collega di entrambi per alcuni anni, come autore di $c a-$ sus e quaestiones, anche disputatae, in tema di poteri riconosciuti al giudice, di false testimonianze, di legati testamentari e di norme previste dagli statuti padovani ${ }^{13}$.

chanore di S. Lucia da Padova, Guido a Ferris da Mantova, Egidiolo da Cremona decretorum doctoribus, Niccolò Mattarelli, Oldrado da Lodi e Francesco Mattarelli legum doctoribus, omnibus salariatis et actu regentibus in studio paduano. Atto ripetuto nella sostanza, ma con data diversa (1308 ottobre 6), ibidem, p. 67, ma che Colle, Storia scientifico-letteraria dello Studio di Padova, p. 82, nota b, considera diverso dal precedente. Riccardo, nato a Cremona intorno al 1259, insegna diritto civile a Padova dal 1289 al 1310-11 (GIAzZI, Riccardo Malombra, p. 66), Oldrado dallo scorcio del secolo XIII al 1310, prima di trasferirsi ad Avignone l'anno successivo come uditore della Sacra Rota (Murano, Oldrado da Ponte, p. 80 s.).

10 Gloria, Monumenti della Università di Padova, p. 71 (1310 luglio 25): conferimento del dottorato in diritto civile a Giovanni Giroldi da Losanna, presentato da Niccolò Mattarelli e da altri doctores padovani non specificati; sono presenti, accanto ai rettore degli scolari ultramontani e citramontani, Riccardo Malombra, Oldrado da Ponte, Francesco Mattarelli modenese e Paolo de Sulimano bolognese legum doctoribus, al fianco di Egidiolo da Cremona e Guido a Ferris da Mantova decretorum doctoribus, omnibus salariatis et actu regentibus.

11 Marangon, Gli Studia degli ordini mendicanti, n. 2, p. 101 (12 febbraio 1305); breve profilo in Gloria, Monumenti della Università di Padova, p. 260 s. e VICINI, Di Nicolò Mattarelli, p. 27 s., che ne ricorda l'insegnamento a Padova soltanto fra 1308 e 1310.

12 ASMo, Memoriale antico, a. 1309, n. 598 (1309 aprile 3): in Padova Niccolò e il figlio Francesco, anch'egli lettore nello studio cittadino, dichiarano di non avanzare ulteriori richieste nei confronti, rispettivamente, del fratello e dello zio Alessandro ex vigore cuiusdam instrumenti scripti per Albertum Bidellum notarium; tra i testimoni compare anche Diocles de Mattarellis.

13 Guglielmo Durante, Speculum iudiciale, II, III, De fructibus et interesse, add. Predictam legem, t.1, p. 913: «Ex quo in materia fructuum legata miscuimus occurrit quaestio, dum ibi erant Paduae, disputata per dominum Franciscum de Mattarelli [...]»; III, I, De accusatione, add. Ut intra certum tempus, t. 2, p. 21: «[...] duae tenent quaestiones, et praesupposito statuto, quod quaerelae bannitorum non audiantur et quod impune possit offendi, pono bannitum pro rapina accusari de homicidio, qui securatus per iudicem venit; deposuerunt testes contra eum, quos accusat de falso; quaeritur an per statutum repellatur accusatio? Et haec fuit Francisci de Mattarellis et incipit 'Statutum est Paduae' [...]»; III, I, De inquisitione, add. Terminum praefigat, t. 2, p. 36: «Et sciendum quod Franciscus Mattarelli in quaestio quae incipit 'Iudex habet merum' refert fratrem [scil. patrem] suum dominum Nicolaum tenuisse, ut supra, quod iudex super utraque procedit [...]»; IV, III, De obligationibus et solutionibus, add. secundum Albertum Galleotum, t. 2, 
Dall'avere svolto il ruolo di promotore nei confronti di alcuni allievi padovani traggono spunto un paio di sermoni pronunciati in pubblico da Niccolò Mattarelli e tráditi all'interno di due manoscritti fiorentini ${ }^{14}$, uno dei quali si segnala per alcuni motivi di indubbio interesse inerenti sia il profilo cronologico che contenutistico. L'orazione viene pronunciata da Mattarelli non oltre il maggio $1295^{15}$,

p. 361: «Franciscus de Mattarellis etiam disputavit hunc casum et in idem recidit in sua distinctione prolixa». Alcuni di questi passi sono ricordati in Colle, Storia scientifico-letteraria dello Studio di Padova, p. 82 s.; si veda Sorrenti, Testimonianze di Giovanni D’Andrea, n. 151, p. 95; n. 174, p. 104; n. 179, p. 107; n. 288, p. 143. Nella quaestio «Iudex habens merum imperium», trascritta nel codice vaticano ASP A.29, ff. 261ra-262rb, Francesco richiama anche il parere del padre Niccolò (non del fratello, come erroneamente riportato da Giovanni D’Andrea in Guglielmo Durante, Speculum iudiciale, III, I, De inquisitione, add. Terminum praefigat, t. 2, p. 36). La quaestio è ricordata in Bellomo, I fatti e il diritto, p. 172, note 63 e 64, e in ID., Quaestiones in iure civili disputatae, p. 176.

${ }_{14}$ Biblioteca Nazionale Centrale di Firenze, ms. II.I.64, ff. 155v-157r (edito in MARANGon, Un «sermo pro scolari conventuando», pp. 156-161) e ms. Magl. VI.134, ff. 62r-63r (ff. 65r-66r cartulazione moderna), con varianti che riguardano ortografia, scelta dei termini, trasposizioni di parole e giri di frase. In questo secondo manoscritto alle ff. 61r-62r (ff. 64r-65r cartulazione moderna) è contenuto un altro sermo dottorale attribuito al Mattarelli, non segnalato da Paolo Marangon ma già rilevato in Inventari dei manoscritti delle biblioteche d'Italia, XII, Forlì 19021903, pp. 148-150, con una descrizione sommaria dei contenuti del codice. Per una analoga descrizione del ms. II.I.64 (vecchia segnatura Magl. VI, 144) vedi Inventari dei manoscritti delle biblioteche d'Italia, VIII, Forlì 1898, pp. 25-27. Si tratta di due rilevanti codici miscellanei, realizzati da mani diverse, contenenti sia testi letterari, soprattutto a carattere epistolare e oratorio, sia testi giuridici quali consilia, repetitiones e disputationes, dovuti soprattutto a maestri attivi presso lo Studium bolognese nei secoli XIV e XV. All'interno del ms. II.I.64 si segnalano in specie i modi arguendi di Ranieri da Forlì (ff. 246r-250v) e le raccolte di glossae contrariae al Codice, al Volumen, al Digesto vecchio, all'Inforziato e al Nuovo di Dino del Mugello con le additiones di Ranieri da Forlì (ff. 250v-255v), studiate da Bellomo, Glossae contrariae di Cino da Pistoia, in relazione ad altri codici anche fiorentini come i Magl. XXIX, 172 e 179. Nel codice II.I.64 si leggono due sermones che si possono fondatamente attribuire a Giovanni da Imola, benché pronunciati in un contesto estraneo a quello prettamente didattico, riconosciuti di recente da PADOVANI, Dall'alba al crepuscolo del commento, p. 111 ss. Nel fondo Magliabechiano sono presenti altri codici di contenuto analogo come il Magl. XXIX, 179, che riunisce documenti connessi alla storia bolognese e soprattutto a quella dello Studium, tra cui una serie di sermoni accademici e altri testi giuridici di varia natura: inventario in Piana, Nuove ricerche su le Università di Bologna e di Parma, pp. 56-65.

15 Nel testo viene infatti ricordato il vescovo Bernardo Platon, alla guida della diocesi padovana dal marzo 1287 al maggio 1295: MARANGON, Un «sermo pro scolari conventuando», p. 151 e p. 161 nel testo del sermone. 
nel solco di un genere letterario ben diffuso negli Studia duecenteschi e ancora praticato nel secolo successivo tanto da Cino ${ }^{16}$, Barto$10^{17}$ e dal massimo allievo di questi ${ }^{18}$, ed è largamente intessuta di citazioni letterarie e scritturali, oltre che prettamente giuridiche, che attingendo a un lessico e a similitudini ben collaudate nella tradizione duecentesca ${ }^{19}$ convergono nel celebrare l'ingresso del nuovo dot-

16 Padovani, Un sermo di Cino da Pistoia, ove si pubblica e commenta un sermo pro doctoratu (conservato nel codice vaticano Chigi E.VIII.245, f. 61ra-b) pronunciato da Cino a Bologna tra l'ottobre del 1334 e i primi mesi dell'anno successivo, che costituisce una delle testimonianze più antiche di tale genere letterario.

17 Cfr. Bellomo, Saggio sull'Università, p. 244. Si rammentano i due noti sermoni tenuti da Bartolo in occasione dei dottorati del fratello Bonaccorso e di Giovanni Bentivoglio da Sassoferrato, suo parente, leggibili in BARTHOLUS DE SAXOFERrato, Commentaria cum additionibus Thomae Diplovatatii, ff. 214rb-215ra e f. 215ra-vb. Li ricorda CORTESE, Intorno all'edizione di Bartolo curata dal Diplovatazio, p. 384, e già Quaglioni, Autosufficienza e primato del diritto, p. 129 ss. e ID., I lettori di Bartolo, p. 190, in merito all'affermata superiorità del ius civile sul canonicum, nel quadro di una complessiva eccellenza degli studi legali rispetto a tutte le altre scienze, sottolineata da Bartolo nel discorso pronunciato per la laurea del fratello. Sul punto ritorna più di recente TregGiari, La laurea del giurista, p. 103 ss., sottolineando, in relazione soprattutto all'orazione pronunciata per la laurea di Bonaccorso, la sua valenza «come testo di autorappresentazione della scienza civilistica trecentesca, compiuta attraverso la simbologia del rituale dottorale» (p. 107). Nel contesto padovano, dopo la testimonianza offerta da Mattarelli sullo scorcio del Duecento, la tradizione dei sermones dottorali pronunciati dai maestri in occasione della laurea degli allievi conosce un rilancio dalla seconda metà del secolo XIV: Mantovani, Le orazioni accademiche per il dottorato, p. 79 e passim.

18 Padovani, Sette orationes pavesi pro doctoratu di Baldo degli Ubaldi, ove si pubblica e si commenta un manipolo di discorsi pronunciati dal giurista perugino a Pavia nel decennio 1390-1400 in occasione delle lauree di alcuni dottorandi, traditi dal codice vaticano Barb. Lat. 1410.

19 Già Azzone nel proemio alla Summa Institutionum, sottolineando l'uso, dal parte del princeps, delle armi in tempo di guerra e delle leggi in tempo di pace, valorizza la similitudine tra armi e leggi e la conseguente identificazione tra milites e operatori del diritto: Azonis Iurisconsultissimi in ius civile Summa, f. 278rb: «[...] et tanta gaudent similitudine pariter et splendent utilitate ut nomen armorum et nomina eorum qui exercentur in armis accomodentur legibus et legistis. Leges ergo dicuntur arma et imperator legibus dicitur armari ut in principio huius proemii et milites dicuntur advocati [...]». Oltre che nella costituzione Imperatoriam maiestatem con cui vengono promulgate le Istituzioni nel novembre 533, la stretta correlazione tra leggi e armi quali strumenti di governo del principe e di difesa della respublica è notoriamente sottolineata anche nella costituzione Summa rei publice dell'aprile 529. Il rapporto fra questi tre testi viene già evidenziata da FiTTING, Das Castrense Peculium, p. 538 s. Una recente riflessione sul significato che assume l'identificazione tra giuristi e milites e quindi tra scientia iuris e militia nell'ambito 
tore in una milizia togata posta al fianco di quella armata e distinta dal liber legum, simbolo della sapienza dottrinale infusa dal maestro nell'allievo, anziché dalla spada, richiamata invece come simbolo della dignità militare ${ }^{20}$.

Il discorso, impostato in chiave di diretto e affabile colloquio tra il maturo giurista e il giovane dottore, fa appello a una intima dilectio, a una suavitas et dulcedo nella quale si riflette un rapporto di filiazione intellettuale del secondo rispetto al primo che viene esplicitato tramite l'esempio privilegiato del dialogo con il proprio figlio Francesco. Un riferimento posto in apertura del testo che si può interpretare come espediente retorico finalizzato a rafforzare il modello dell'associazione padre-figlio, diffusa e usuale all'interno di tale letteratura d'occasione ${ }^{21}$, ma che non meno potrebbe documentare l'effettiva cerimonia di laurea dello stesso Francesco Mattarelli, compatibile dal punto di vista cronologico sia con l'esperienza complessiva del padre, sia con la più risalente qualificazione nota dello stesso Francesco come doctor iuris ${ }^{22}$.

della società comunale italiana del Duecento si deve a MenZInger-VAlLERAnI, Giuristi e città, p. 206 ss.

20 Un motivo letterario, che fa leva sulla similitudine tra la dignità dei giuristi e quella dei milites, già collaudato da tempo anche nei sermoni pronunciati dagli studenti in occasione dell'examen publicum per il rilascio ufficiale del titolo dottorale: CONTE, $U n$ sermo pro petendis insigniis, pp. 79, 83. Nel discorso di Mattarelli si ritrova menzione della consegna del liber legum dal maestro all'allievo, che nel secolo XIII «è indicato normalmente come l'unico segno tangibile che accompagnava la licenza di insegnare concessa dal professore al termine dell'esame», e anche dell'imposizione del berretto sul capo del dottorando, un elemento che invece si diffonde nel secolo successivo assieme all'anello e alla toga: ibidem, p. 83 ss. e p. 84 , nota 47 , per la citazione.

21 Come propende Marangon, Un «sermo pro scolari conventuando», p. 152 s., relativamente al richiamo scritturale e al paragone esplicitato in apertura del sermone: «Ecce odor filii mei sicut odor agri pleni, cui benedixit Dominus. Verba ista sunt Ysaac ad Iacob filum suum, Genesis, capitulo.27., que satis convenienter ad presentem sollempnitatem adaptari possunt, ut sicut verba mea ad filium meum. F.» (ibidem, p. 156).

22 Ritenendo Niccolò Mattarelli nato intorno al 1240, si può agevolmente pensare che il figlio si sia addottorato quando il padre aveva circa cinquant'anni. La prima attestazione nota di Francesco quale doctor legum risale infatti al 1293: ASMo, Memoriale antico, a. 1293, nn. 826 e 827 (9 febbraio 1293): Iacopinus de Gorgadelis con la figlia Bartolomea, da una parte, e Zanne del fu Ugolino, dall'altra, affidano a Francesco figlio di Niccolò Mattarelli il compito di giudicare come 


\section{Tracce sparse di attività didattica}

Accanto a queste testimonianze strettamente legate all'ambiente dello Studio padovano e, in specie, a una tappa significativa dell'attività del docente che segna il traguardo conclusivo nella formazione dei propri allievi, è possibile ricostruire almeno in parte la trama del lavoro di Mattarelli connesso al suo insegnamento civilistico.

Tracce di allegazioni attribuite al maestro modenese si ritrovano in una serie di manoscritti contenenti il Codice giustinianeo e accomunati tanto dalla circolazione in area padovana quanto dal fatto di documentare il pensiero di maestri collocati su quella ormai nota "linea alternativa" rispetto all'asse privilegiato della tradizione azzoniano-accursiana che dal magistero bolognese di Ugolino Presbiteri, Iacopo Balduini e Odofredo, nell'arco della prima metà del Duecento, si dipana fino ai primi decenni del secolo successivo attraverso l'attività di noti giuristi quali Roffredo Beneventano, Benedetto d'Isernia, Accursio da Reggio, Alberto di Odofredo, Martino da Fano, Giovanni Fagioli, Guido da Suzzara, Dino del Mugello e Pietro dei Cerniti. Maestri che favoriscono la diffusione delle nuove metodologie anche verso lo Studio di Napoli e altri centri italiani condividendo la tendenza - in linea generale - a staccarsi dall'esegesi dottrinaria tutta interna al perimetro delle leges imperiali per guardare con sempre maggior attenzione agli ordinamenti particolari, al loro diritto e alla miriade di casi concreti che scaturiscono dall'esperienza quotidiana e dal dettato delle norme statutarie e si prestano a essere valutati alla luce delle potenzialità espansive del ius commune.

In questa serie di manoscritti largamente circolanti in ambienti scolastici è rilevante il codice rumeno Alba Iulia II.4, dotato di apparato azzoniano e fittamente utilizzato sino ai primi del Trecento. Conserva infatti tracce di letture tenute nelle scuole, oltre che da giuristi di minor caratura, da affermati docenti quali, accanto a Mattarelli23 ${ }^{23}$ Iacopo Balduini, Benedetto d'Isernia, Giovanni Fagioli,

arbitro una questione dotale sorta tra loro; e in pari data il doctor legum pronuncia il lodo richiesto.

23 DolezaleK, Repertorium manuscriptorum Codicis Iustiniani, pp. 91-97 per la descrizione analitica del codice, in part. p. 96: sono presenti additiones siglate ni. de Matarelis ai ff. 94v e 103v, e anche a f. 160va, ove un ignoto scolaro 
Simone Vicentino, Iacopo d'Arena, Federico delle Scale e Riccardo Malombra ${ }^{24}$, attivi per la maggior parte a Padova tra il tardo secolo XIII e i primi decenni del Trecento ${ }^{25}$. L'uso prolungato in ambiente padovano è confermato dal fatto di attestare l'attività forense del giudice e dotto podestà Lovato de' Lovati, nato a Padova intorno al 1240 - quindi contemporaneo di Mattarelli -, e del trevigiano Varnerius de Falchis, sapiente della curia della propria città nel $1315^{26}$. Tra i possessori del codice spicca anche il giudice Henrigetus, che lo acquistò nel 1281 e poi negli anni Venti del Trecento svolse l'incarico di iudex vicarius di Guecellone da Camino a Serravalle, nel Trevigiano ${ }^{27}$.

Altre allegazioni in grado di documentare riferimenti al pensiero di Mattarelli si rinvengono nel codice Augsburg 2.52, dotato di apparato accursiano e risalente al secolo XIV, contenente le sigle

dichiara di avere ascoltato la lectura del maestro rilevando la consonanza della sua opinione con quella di Martino da Fano. L'additio è trascritta in parte in SPECIALE, La memoria del diritto comune, p. 86, nota 24. Si veda anche DolezAleK, The Lectura Codicis of Odofredus, p. 105 ss e in part. p. 110 ss., ove trascrive le serie di circa 60 additiones riferite a Iacopo Balduini e riportate nel codice Alba Iulia II.4 a margine del secondo libro del Codex, che in alcuni casi si devono a studenti direttamente presenti alle lezioni del maestro bolognese.

24 Speciale, La memoria del diritto comune, pp. 80 s., 84 ss., 96 ss., 239 s. Il manoscritto è ricco anche di glosse in cui viene richiamato il pensiero di numerosi altri giuristi attivi tra XII e XIV secolo quali Irnerio, Piacentino, Giovanni Bassiano, Ugolino, Accursio, Odofredo, Omobono da Cremona, Francesco d'Accursio, Dino, Martino Sillimani, Bartolomeo da Cornazzano e del meno noto Conversano di Leone da Viterbo; riporta anche una nutrita serie di quaestiones ex facto emergentes e di quaestiones statutorum, oltre a «un reticolo grafico di glosse di Jacopo Baldovini e un diverso reticolo grafico che testimonia parti di lecturae ricche di quaestiones di Alberto Papiense, Uberto da Bobbio, Alberto Galeotti, Jacopo d'Arena, Accursio da Reggio, Guido da Suzzara» (ibidem, p. 97).

${ }_{25}$ Per i dettagli della residenza in Padova e dell'attività di insegnamento si rinvia ai profili biografici di LABARDI, Malombra Riccardo (a); ID., Malombra Riccardo (b); Giazzi, Riccardo Malombra; Quaglioni, Dell'Arena (D’Arena) Iacopo; ID., Iacopo d'Arena; ID., Delle Scale Federico; Menzinger, Federico della Scala (delle Scale).

26 Speciale, La memoria del diritto comune, p. 88 e anche p. 99 s.; KoHL, Lovati Lovato.

27 Sul giudice-cronista e i suoi rapporti con il codice Alba Iulia II.4: SPECIALE, Henrigetus magistri Gerardi giudice e cronista, ripreso sul punto in questione in ID., La memoria del diritto comune, p. 102 ss. Di Henrigetus nel codice sono anche riportate una distinctio a f. $23 \mathrm{v}$ e una quaestio de facto: DolezAleK, Repertorium manuscriptorum Codicis Iustiniani, p. 95. 
di 26 maestri da Iacopo Baldovini a Federico di Ranieri Arsendi da Forlì. Il codice non fornisce indicazioni circa il suo uso in corrispondenza di rilevanti centri di studio nel panorama italiano del DueTrecento come Bologna, Padova o Napoli, ma soltanto sull'attività didattica di singoli giuristi, come per esempio Accursio da Reggio e la sua scuola, documentando nel complesso la consistente vitalità della 'linea alternativa' nel secolo successivo agli anni Trenta del Duecento ${ }^{28}$.

A questi si può accostare anche il codice Ambrosiano E.25 inf., un pregevole esemplare miniato databile al secolo XIV con apparato accursiano e una consistente trama di additiones contenenti le sigle di 26 giuristi da Bulgaro a Raffaele Fulgosio, tra i quali non mancano Iacopo Baldovini, Odofredo, Martino da Fano, Iacopo d'Arena, Guido da Suzzara, Francesco d'Accursio, Simone Vicentino, Dino, Oldrado, Iacopo Bottrigari e ancora, limitatamente, Mattarellii ${ }^{29}$. Particolarità di tale manoscritto che ne conferma l'utilizzo in ambito padovano è la nota a f. 1r, conforme a una prassi affermatasi circa tra gli anni 1350 e 1475, che attesta l'ingresso in città del codice e la conseguente esenzione dal pagamento del dazio o dall'inoltro di una specifica richiesta a un pubblico ufficio in favore di studenti e maestri che se ne servissero unicamente per uso proprio ${ }^{30}$. Non unico il caso di Padova, per l'applicazione di tale procedura in rapporto alla circolazione del libro

28 Speciale, La memoria del diritto comune, p. 125 s. e anche pp. 219, 230, 242.

29 Ibidem, pp. 140, 220, 231, 280 s. PAce, Riccardo da Saliceto, p. 151 s., al quale si deve l'esame, in particolare, delle testimonianze ascrivibili a un corso di Iacopo Bottrigari e al suo prestigioso allievo, Riccardo da Saliceto, che attestano la circolazione del manoscritto in ambienti didattici bolognesi e padovani. Breve scheda del codice in Cipriani, Codici miniati dell'Ambrosiana, p. 235, che lo attribuisce al secolo XIV e lo definisce di «scuola bolognese». Una più analitica descrizione alla URL http://manus.iccu.sbn.it/opac_SchedaScheda.php?ID=35991, ove si conferma a f. 196vb un'unica additio attribuita a Mattarelli, che risulta collocata a margine di C. 6.37 .8 (De legatis), nel limitato spazio bianco fra testo e Glossa, ed è firmata Nicollaus Matarelli de Mutina.

30 Note di conduxit sembrano rilevabili a f. 1r malamente rifilato (Speciale, La memoria del diritto comune, p. 139). Su tale pratica e la definizione dell'arco di tempo in cui risulta concretamente in uso rimangono fondamentali gli studi di GARGAN, L'enigmatico «conduxit»; ID., Nuovo codici "condotti" a Padova; ID., Le note «conduxit»; ID., Un nuovo elenco di note «conduxit», ove tuttavia non compare mai censito il manoscritto ambrosiano. 
giuridico a uso di docenti e allievi dello Studio locale; anche in altri centri urbani come Bologna e Imola, in base a specifiche disposizioni statutarie, i codici manoscritti sia in entrata che in uscita dalla città vengono sottoposti a dazio tranne che per quelli condotti da studenti, e con la varia aggiunta di ulteriori clausole restrittive ${ }^{31}$.

Legato strettamente allo Studio di Padova è ancora il codice Palatino latino 759, contenente il testo dei primi 9 libri del Codex giustinianeo corredato di apparato accursiano e rintracciabile a cavallo fra Tre e Quattrocento nelle mani del facoltoso studente siciliano Cosimo Veronesi, che lo acquista a Pavia il 1 luglio 1398 e poi lo introduce in Padova il 19 dicembre $1407^{32}$. Il manoscritto, nel quale si rinvengono sparse glosse e additiones chiuse dalle sigle di una trentina di maestri, da Azzone a Raffaele Fulgosio, circola nella scuola bolognese di Iacopo Bottrigari senior nella prima metà del secolo XIV e incorpora riferimenti ai nomi di altri giuristi del medesimo ambiente come Riccardo da Saliceto e Maccagnano degli Azzogui$\mathrm{di}^{33}$. Da Bologna il codice sembra passare a Padova, ove servirà per la trascrizione di alcune additiones del Fulgosio, una delle quali incorpora l'ormai lontana opinione di Mattarelli, recuperando anche una vecchia glossa dello stesso giurista modenese ${ }^{34}$.

Saltuarie tracce della sua attività scolastica provengono da altri due manoscritti sempre contenenti il Codice con apparato accursiano. Il Paris lat. 4533 riporta le sigle di 27 giuristi da Iacopo Baldovini a Bartolomeo da Saliceto e reca tracce anche dell'insegnamento di Johannes de Fabrica, professore a Tolosa nel $1409^{35}$. Il Vaticano

31 Per alcuni esempi e il richiamo alle specifiche norme statutarie si veda $\mathrm{P}_{\mathrm{A}}$ Dovani, Dall'alba al crepuscolo del commento, p. $21 \mathrm{~s}$.

32 Gargan, Nuovi codici "condotti" a Padova, p. 42; Pace, 'Cosmas de Veronisiis de Sicilia', p. 193 ss.: Cosimo acquista sempre a Pavia anche un esemplare del Digesto Vecchio (ms. Palatino Latino 731) e dell'Inforziato (ms. Palatino Latino 742). Speciale, La memoria del diritto comune, p. $138 \mathrm{~s}$.

33 Ibidem, pp. 69, 204 s., 222, 232, 324; PACE, Riccardo da Saliceto, pp. 148 s., 169 ss.

34 Ibidem, p. 185 (la citazione di Mattarelli è a f. 284va) e p. 189 s. per l'attribuzione a Nicolò Spinelli di alcune additiones siglate 'Nic.', 'Ni.' o 'Nich.'; BeLLomo, Giuristi bolognesi al tempo di Taddeo Pepoli, p. 315 s.: la glossa attribuita a Mattarelli è a f. 176rb.

35 Speciale, La memoria del diritto comune, pp. 147, 155n, 221, 232, $297 \mathrm{~s}$. Cfr. MeIJERs, Etudes d'histoire du droit, III, ad indicem. 
Latino 1428 ha circolazione soprattutto in ambiente napoletano e attesta una folta presenza di giuristi attivi nelle scuole locali tra Due e Trecento, da Roffredo Beneventano a Nicolò Spinelli e Giovanni Petrucci ${ }^{36}$. Nel complesso il codice annovera i riferimenti a 35 giuristi da Ottone da Pavia a Baldo e Bartolomeo da Saliceto e l'unica attestazione di Nicolaus de Mactarellis emerge a f. 196v come sottoscrizione a una glossa di commento al testo del Codice ${ }^{37}$.

In merito all'insegnamento svolto dal giurista modenese una testimonianza di assoluto rilievo è offerta dal codice padovano 941, un celebre esemplare del Digesto vecchio databile alla prima metà del secolo XII conservato alla Biblioteca Universitaria di Padova e proveniente dal convento dei frati Eremitani di S. Agostino, ove è attestato con sicurezza dal secolo XIV ${ }^{38}$. Il manoscritto rappresenta uno dei più risalenti testimoni della versione prebolognese della prima parte del Digesto (fino a D. 24.3.1 incluso) e occupa una po-

36 Speciale, La memoria del diritto comune, pp. 142 ss., 207, 222, 228, 297 s. Si veda anche la descrizione analitica del manoscritto in KuTTNER-Elze, A Catalogue of Canon and Roman Law Manuscripts, I, pp. 228-230.

37 Sella, Sigle di giuristi, p. 197, ove si segnala pure la sigla 'Ni. de Ma' a margine di f. $72 \mathrm{r}$ del manoscritto Vaticano latino 2619, contenente la lectura di Bartolomeo da Saliceto al VI libro del Codice giustinianeo con sparse additiones marginali dovute a numerose mani differenti: KuTtNER-Elze, A Catalogue of Canon and Roman Law Manuscripts, II, p. 184. Bevilacoun, Una «quaestio» di Niccolò Mattarelli, p. 142, nota 3, ove ricorda la presenza anche nel Palatino latino 735, a f. 21r, di glossule al Digestum Vetus con Glossa accursiana sempre riconducibili a Mattarelli. Anche in quest'ultimo codice, databile al XIV secolo, a f. 1r-v è documentata la nota conduxit che ne attesta l'introduzione in Padova nel 1393 da parte di Donatus de Imola e anche, in data imprecisata, da parte dell'abate del monastero toscano di S. Maria di Prataglia; il manoscritto è utilizzato da Giacomo Pace nella sua ricerca delle testimonianze dei giuristi che animarono le fasi aurorali della Scuola bolognese: PACE, 'Garnerius Theutonicus', p. 124, nota 1.

38 Descrizioni aggiornate del codice, con riferimenti agli altri testimoni del Digesto vecchio confrontabili con esso (Vaticano Latino 1406 e 1739 (frammento), Parigi 4450, Lipsia 873), in SANTONI, Copisti-editores di manoscritti giuridici, $\mathrm{p}$. 243, nota 56; NicolaJ, Documenti e libri legales a Ravenna, p. 785 s.; GNAN-TonioLo, Splendore nella Regola, p. 75 s. Sul manoscritto padovano vedi anche Toniolo, Frati maestri e libri miniati a Padova, p. 580 s. Al testo del Digesto vecchio è stato premesso un bifoglio contenente, in grafia duecentesca, l'indice dei titoli sino a D. 24.2 (f. 1r) seguito dal testo della costituzione Omnem (ff. 1v-2v). Sul margine superiore di f. $1 \mathrm{v}$, al di sopra della rubrica premessa alla costituzione giustinianea, è riportato il seguente distico ancora in auge in età umanistica: Dat Gallienus opes et sanctio iustiniana / ex alliis (sic) paleas, ex istis collige grana. Cfr. AnTonAzzi, Lorenzo Valla e la polemica sulla donazione di Costantino, p. 65. 
sizione importante nella tradizione testuale di questa difficile parte dei libri legales ${ }^{39}$. Il codice ha attirato l'attenzione degli studiosi anche per le catene di segni in minio apposti sui margini del testo, a doppia colonna, letti in un primo tempo come segni di paragrafatura del Digesto tracciati su un esemplare in origine privo di suddivisioni interne e senza rinvii speculari nell'interlinea del testo, ma più di recente interpretati come rinvii a passi paralleli e a riferimenti testuali reciprocamente connessi grazie a un ventaglio di confronti assai più esteso dal punto di vista sia quantitativo che tipologico ${ }^{40}$.

Il codice è costellato da una fittissima trama di annotazioni interlineari e marginali che attestano un minuzioso lavoro interpretativo svolto nell'arco di varie generazioni con interventi di mani diverse databili dal XII al XIV secolo in cui si possono distinguere quattro strati principali. Accanto a glosse chiuse con sigle riferibili a Irnerio ${ }^{41}$ se ne rilevano altre che rinviano a maestri bolognesi delle prime generazio-

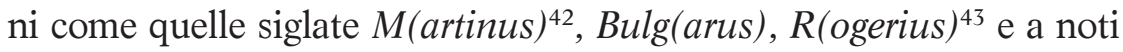

39 In merito si rinvia alle analisi filologiche di PESCANI, La posizione del $\mathrm{V}$ 1406 e ID., La scoperta del 'Bononiensis', corrette qualche anno dopo da MüLLER, The Recovery of Justinian's Digest. Più di recente, l'intera problematica relativa alla tradizione del codice padovano rispetto all'archetipo del Digesto giustinianeo e alla versione offerta dalla Littera Florentina è riconsiderata in RADDING-CIARALLI, The Corpus iuris civilis in the Middle Ages, p. 171 ss.

40 Mor, La divisione in paragrafi delle leggi del Digesto, in part. pp. 147 s. e 150 per i riferimenti al codice Padova 941; Dolezalek-Weigand, Das Geheimnis der roten Zeichen, passim.

41 Besta, L'opera di Irnerio, p. IX ss.: il manoscritto padovano è uno dei tre utilizzati per l'edizione delle glosse irneriane.

${ }_{42}$ Ai ff. $11 \mathrm{v}, 39 \mathrm{v}, 83 \mathrm{r}, 87 \mathrm{v}, 135 \mathrm{v}, 174 \mathrm{r}$.

43 Nicolat, Documenti e libri legales a Ravenna, p. 785 s.: come già BESTA, L'opera di Irnerio, p. IX, distingue quattro principali mani che hanno lavorato sul codice redigendo le glosse chiuse, oltre che dalla sigla di Irnerio, da quelle che rinviano a Bulgaro, Rogerio e molte altre del secolo XIII «fra le quali colpiscono quelle segnate gy. (Giovanni Ispano: Bologna e Padova), g. de suça (Guido da Suzzara: Modena, Padova...), dominus Nicholaus o Ny. (Niccolò Mattarelli, XIII-XIV sec.: Modena-Padova)» (p. 786). La sigla Bulg(arus), in particolare, si rileva ai ff. 93v, 179v; quella $R$ (ogerius) ai ff. 3r, 7r, 8r, 14r-v, 15r, 16r-v, 17r, 20r-v, 21r, 37r-v, 55r, 102r, 134v, 137r, 143v, 144v, 149r, 159r, 175r. Diversamente BESTA, op. cit., p. XI, attribuiva la sigla $N y$. a Nicolò Furioso, allievo di Giovanni Bassiano attivo a Bologna fino al primo decennio del Duecento. Una significativa testimonianza extrabolognese a margine di D. 16.3.24 concerne un esempio in materia di interessi conseguenti al deposito di una cospicua somma di denaro riferito a Burgundione pisano (f. 139r). 
giuristi di epoca successiva tra i quali Azzone ${ }^{44}$, Uberto da Bobbio ${ }^{45}$, Jacopo d'Arena ${ }^{46}$, Odofredo ${ }^{47}$ e ancora, fatta eccezione per le numerosissime glosse seguite dalla sigla di Accursio, a Guido da Suzzara ${ }^{48}$, a Martino da Fano ${ }^{49}$ e soprattutto a Niccolò Mattarelli ${ }^{50}$. Glosse e additiones a lui riferite si distendono per tutta l'ampiezza del testo e in alcuni casi appaiono rafforzate dall'interesse del reportator a sottolineare in forma esplicita l'orientamento del maestro (placet domino Nicolao, f. 56r; dominus Nicho tenet contrarium, f. 90v; secundum Ny, ff. $20 \mathrm{v}, 81 \mathrm{r}, 142 \mathrm{v}$ ) manifestando un'attenzione puntuale e diretta che lo avvicina strettamente all'ambiente della sua scuola. Un orientamento che si coglie anche quando una breve additio riferita a Mattarelli va a integrare la prima di una serie di tre quaestiones di Guido da Suzzara in materia di arbitrato sviluppate a margine di D. 4.8.21.10 (f. $49 \mathrm{v})$ : l'amanuense è il medesimo, attento a riportare per esteso i casi proposti dal maestro reggiano assieme alla riflessione integrativa del collega modenese, mentre il comune ambiente emiliano famigliare ai due giuristi si riflette nelle fattispecie proposte da Guido in merito ad arbitri che si figurano attivi sia in civitate Mutine sia in civitate Regii.

Eccettuati Jacopo d'Arena, che insegna a Padova dai primi anni Sessanta del Duecento, e l'irrequieto Guido da Suzzara, che soggiorna nella città veneta soltanto per un biennio fino al 1266, i giuristi

44 Aç: ff. 11r, 55r, 65r, 177r, 182v. A f. 183r all'interno di una glossa chiusa con la sigla di Accursio si rinvia all'opinione contenuta in libro domini Azonis.

45 Ub., Ubertus: ff. 49v, 96v.

46 Iac., Ia. de Ar.: f. 120r, 195v (et hoc placuit domino Ja. de Ar.).

47 Odoff., Odd., Odo: ff. 54v, 86r, 111r, 139v.

48 G., Gu., Gui., Gui de Suç., G. de Suça: ff. 28r, 49v, 50r-v, 72r, 77r, 85v, 117r. A Guido da Suzzara rinvia pure una glossa isolata vergata sull'ultimo foglio del manoscritto $(198 \mathrm{v})$, contenente una nota di possesso trecentesca che fa riferimento al convento padovano dei frati Eremitani di S. Agostino e un arbor parentelae schizzato a penna, nella quale si concentra un suo insegnamento venato da istanze molto pratiche: Dominus Guido de Suzaria dicebat quam tres erant leges in iure utiliores: aliis prima est $C$. de iudiciis l. properandum (C. 3.1.13), alia est ff. de re iudicata l. a divo Pio (D. 42.1.15), alia est ff. ex quibus causis in possessione eatur l. Fulcinius (D. 42.4.7).

49 M.F., M.Fa., Ma., Ma.Fa., Ma. de Fano: ff. 6v, 7r, 8v, 9r-v, 14r, 34v, 44r, $47 \mathrm{v}, 53 \mathrm{v}, 90 \mathrm{r}-\mathrm{v}, 100 \mathrm{r}, 108 \mathrm{r}, 141 \mathrm{r}$.

$50 \quad N y .:$ 1v, 12r, 14r, 15r, 16r, 18v, 20v, 23r, 28r, 30v, 32r, 37r-v, 49r-v, 53r, 59r, 65v, 66r, 67v, 71r, 78v, 81r, 83v, 90v, 92r, 93v, 102r-v, 114v, 116r, 118r, $128 \mathrm{r}, 130 \mathrm{r}, 134 \mathrm{r}, 158 \mathrm{v}, 159 \mathrm{v}, 167 \mathrm{r}, 180 \mathrm{v}, 181 \mathrm{v}, 188 \mathrm{r}, 191 \mathrm{v}, 193 \mathrm{r}$. 
maggiormente rappresentati nel codice 941 seguono percorsi estranei allo Studio padovano che rinviano a una circolazione in sedi emiliane quali, oltre a Bologna, soprattutto Modena e Reggio, ove peraltro insegna anche Guido tra gli anni Sessanta e Settanta. A Padova, tuttavia, riporta una lunga glossa chiusa dalla sigla di Francesco Mattarelli (f. 18r) che prova una continuità d'uso del manoscritto rispetto agli anni di insegnamento del padre Niccolò, quando egli era seguíto da reportatores molto attenti alle sue lezioni e interessati a segnalare anche il contrasto rispetto alle posizioni di altri giuristi, come - con un ulteriore esempio - nel caso di Martino da Fano e della sua interpretazione di una norma penale contenuta negli statuti di Ferrara (f. 90v $)^{51}$.

A un diverso circuito di utilizzo appartiene invece un esemplare miniato del Codex Iustiniani completo di apparato accursiano databile alla prima metà del secolo XIV e conservato a Tubinga. Non appare di largo uso in ambito scolastico, dal momento che riporta una serie ristretta di additiones attribuite unicamente a Mattarelli52.

Le testimonianze ora ripercorse non si rivelano abbondanti, ma, a parte quest'ultimo caso isolato, convergono nel documentare una circolazione di allegazioni riconducibili a Niccolò Mattarelli in stretta associazione con gli esponenti di una linea interpretativa che dal tempo di Iacopo Balduini viene via via arricchita da richiami all'insegnamento di Odofredo, Iacopo d'Arena, Francesco d'Accursio e Guido da Suzzara, tutti maestri sempre attestati nella serie di manoscritti sopra richiamati ${ }^{53} \mathrm{e}$ in buona parte documentati, assieme a

51 Si segnala anche un notabilis a f. $15 \mathrm{r}$ in materia feudale (Intellige ut in usibus feudorum si vasallus feudo privatur, cui debeat deferri. lex unica. Ny) a margine di un frammento di Ulpiano incluso nel titolo de in ius vocando (D. 2.4.8.2). Il richiamo diretto è a LF II, 31 (= Lehmann, Consuetudines feudorum, p. 249).

52 Tübingen Mc 294. È ricordato da SPECIALE, La memoria del diritto comu$n e$, pp. 159, 319. Per la descrizione del codice, che per grafia e gusto iconografico pare redatto in Francia nella prima metà del Trecento, si veda BRINKHUS-MeNTZELREUTERS, Handschriftenkatalog der Universitätsbibliothek Tübingen, p. 183 s., con richiami anche alle pp. 424, 444, 448. Una più sintetica scheda del codice in EBEL, FIJAL, Kocher, Römisches Rechtsleben im Mittelalter, p. 196.

53 Sono frequenti pure le allegazioni del pensiero di Cino da Pistoia e Iacopo Bottrigari (Augsburg 2.52, Milano E.25 inf., Paris lat. 4533, Palatino lat. 759, Vaticano lat. 1428), di Riccardo Malombra (Alba Iulia II.4, Augsburg 2.52, Milano E. 25 inf., Palatino lat. 759, Vaticano lat. 1428), Dino del Mugello (Alba Iulia II.4, Milano E.25 inf., Palatino lat. 759, Vaticano lat. 1428), Iacopo Belvisi (Augsburg 2.52, Milano E.25 inf., Paris lat. 4533, Vaticano lat. 1428) e Bar- 
Mattarelli, anche nelle serie di additiones al Digesto vecchio presenti nel codice Laurenziano $158^{1}$, che in più ne testimonia la circolazione in ambienti scolastici sensibili all'insegnamento di noti giuristi ultramontani quali Guglielmo de Plaisian, Guglielmo de Cuneo, Jacques de Révigny, Pierre de Belleperche e Pietro de Podiodorphila ${ }^{54}$.

\section{Repetitiones $e$ tractatus}

Una significativa presenza di Mattarelli in stretta relazione con il circuito di giuristi maggiormente rappresentativi di un indirizzo dottrinario più flessibile e libero rispetto alla tradizione azzonianoaccursiana viene confermata tramite il legame con Guido da Suzzara documentato dal manoscritto Madrid, Biblioteca Nacional, 823. Si tratta di un codice miscellaneo ove, accanto a opere canonistiche e civilistiche, sono trascritte le supleciones di Guido al Codice e al Digesto vecchio (ff. 89ra-129ra e 129rb-152rb) ${ }^{55}$ seguite da una colla-

tolomeo da Saliceto (Augsburg 2.52, Milano E.25 inf., Paris lat. 4533, Palatino lat. 759).

54 Firenze, Biblioteca Medicea Laurenziana, Acquisti e Doni $158^{1}$, con additiones riferite a numerosi giuristi operanti fra la metà del Duecento e i primi decenni del Trecento; accanto ai maestri francesi si rilevano riferimenti a Dino del Mugello, Cino da Pistoia, Guido da Suzzara, Jacopo d'Arena, Iacopo Belvisi, Martino da Fano, Odofredo e Uberto da Bobbio: MAFFEI, Qualche postilla alle ricerche di E.M. Meijers, in part. p. $97 \mathrm{~s}$.

55 Descrizione del codice, composto da 158 fogli e risalente al sec. XIV, in Inventario general de manuscritos de la biblioteca nacional, II (501-896), Madrid 1956, p. 421 s., ove non sono individuati i testi attribuiti a Mattarelli, confusi con la parte finale dell'opera di Guido, e non ne viene riconosciuto l'autore. In merito si veda Martino, Ricerche sull'opera di Guido da Suzzara, pp. 15 ss., 31 ss. per osservazioni sulla natura e le caratteristiche delle supleciones contenute nel manoscritto madrileno, frutto di un complesso di appunti presi da un anonimo allievo di Guido durante le lezioni da lui tenute. Gli altri testi inclusi nel codice sono un ordo iudiciarius composto da Tancredi (ff. 1ra-12vb); una serie di quaestiones dominicales seu sabatine di Bartolomeo da Brescia seguite dalla Summa super titulis decretalium a lui attribuita (ff. 13ra-35vb, 36ra-54va); i Praeludia et exceptiones di Uberto di Bonaccorso (ff. 54va-74va); una Summa super feudis attribuita a Oddone (ff. 74va-88vb). Cfr. Schulte, Die Geschichte der Quellen und Literatur des canonischen Rechts, I, p. 203 s.; II, p. 86. Un esemplare dei Praeludia di Uberto di Bonaccorso è contenuto nel codice della Biblioteca dell'Archiginnasio B.2794, ai ff. 123r-128v: cfr. NicolinI, Trattati 'de positioni- 
na di repetitiones - forse frammenti di più ampie lecturae - ad alcune leggi sempre del Codice e del Digesto vecchio identificate con la sigla finale di Mattarelli; testi che, accanto alle collane di quaestiones confluite nei due ben noti libri magni vaticani, rappresentano il complesso più organico di riflessioni del maestro modenese più direttamente collegato all'attività didattica da lui svolta ${ }^{56}$. Di quest'ultima potrebbe essere frutto diretto la lectura - verosimilmente al Codice - più volte richiamata da Baldo e da lui tenuta largamente presente nei propri Commentari, in specie quello al VI libro del Codice giustinianeo ${ }^{57}$.

Conferma della circolazione abbastanza larga di materiali derivati dall'attività di insegnamento svolta da Mattarelli è data dalla disponibilità di ulteriori testimoni di due tra le repetitiones incluse nel manoscritto madrileno, dedicate rispettivamente ai giudizi d'appello ${ }^{58}$ e alla consuetudine ${ }^{59}$, diffuse in esemplari

bus' attribuiti a Martino da Fano, p. 26 s. Edizione a stampa in CASELLI, Corpus glossatorum juris civilis, V.3.

56 Già Martino, Ricerche sull'opera di Guido da Suzzara, p. 15 s., nota 24, individua la serie di testi attribuiti a Mattarelli, ma l'ulteriore esame del manoscritto aiuta a precisarne il riconoscimento. Si tratta di repetitiones - interpretabili anche come frammenti di più ampie lecturae - ad alcune leggi incluse nei libri 2,1 e 7 del Codice giustinianeo e nel libro primo del Digesto, copiate ai ff. 152va-158vb nel seguente ordine: ad C. de edendo (C. 2.1), ove sono esaminate la 1. ipse despice (C. 2.1.1), la l. is apud quem (C. 2.1.2) e la l. qui accusare volunt (C. 2.1.4); in calce alle singole parti del testo si rilevano le sigle ni. de m. (f. 152va); ni.m. (f. 152vb); ni.m. (f. 152vb); ni.m. (152vb). Ad C. de pactis. l. si quis (C. 2.3.29) in calce al testo, che si presenta frammentario, la sigla ni. (f. 153ra). Ad C. de edendo, 1. edita actio (C. 2.1.3); in calce la sigla ni. (f. 154ra). Ad D. de legibus et senatusconsultis, 1. de quibus (D. 1.3.32); a margine del testo la nota de Matarello (f. 156rb). Ad C. de episcopis et clericis, l. nulli licere (C. 1.3.28); in calce la sigla ni. (f. 157ra). Ad C. qui et adversus quos in integrum restitui non possunt, 1. in consilio (C. 2.41.1); in calce la sigla ni. (f. 158rb). Ad C. de temporibus appellationum (C. 7.63), con testo incompleto.

57 Per gli specifici riferimenti vedi sotto, cap. III, par. 4.

58 La repetitio ad C. de temporibus appellationum (C. 7.63) è riportata in altri due manoscritti: ASP A.29, ff. 292ra-294ra e La Seu D’Urgell 2109, ff. 164ra$167 \mathrm{rb}$, per i quali vedi cap. II, nota 127.

59 La repetitio ad D. de legibus et senatusconsultis, 1. de quibus (D. 1.3.32) è nota, nella forma di trattato autonomo, anche tramite i seguenti manoscritti: Roma, Vittorio Emanuele 1511, ff. 180va-182ra, indicata nell'indice analitico a f. 188vb come Tractatus consuetudinis per ny(colaum) de mat(tarellis); München, Codices Latini Monacenses 26669, ff. 283v-292v; Stuttgart, Jur. fol. 123, ff. 12vb-19ra; Tübingen Mc 299, ff. 43ra-48ra. Sul noto codice romano, riconducibile per gran parte a un'origine bolognese e con tracce d'uso che lo legano a Perugia e ad altre vicine città umbre, si veda sotto, cap. II, note 132-133. All'interno del codice mo- 
riprodotti in codici oggi conservati in area iberica, italica e germanica.

In tempi pressoché corrispondenti all'arco dell'attività didattica e scientifica di Mattarelli, la riflessione sulla consuetudine viene sviluppata anche dai maestri orleanesi e sia Jacques de Révigny che Pierre de Belleperche elaborano repetitiones sulla medesima legge del Digesto vecchio, individuata per lunga tradizione come una delle sedes materiae privilegiate per fornire un inquadramento dottrinale all'argomento ${ }^{60}$. Rispetto a essi, il giurista modenese procede su una linea indipendente innestata nel solco della tradizione interpretativa di matrice bolognese, e la sua riflessione è giudicata di notevole interesse come anello di congiunzione tra i glossatori e i successivi commentatori italiani del Trecento ${ }^{61}$.

La repetitio-tractatus di Mattarelli, conosciuta da Alberico da Rosciate $^{62}$ e anche da Cino e da Angelo degli Ubaldi ${ }^{63}$, si pone su una

nacense il tractatus-repetitio del maestro modenese è segnalato in HALM-MAYER, Catalogus codicum latinorum Bibliothecae Regiae Monacensis, p. 201. Relativamente al codice di Stoccarda, l'opera di Mattarelli era già registrata nel catalogo manoscritto approntato da E. SECKEL, Verzeichnis der mittelalterlichen Rechtshandschriften der königlichen Bibliothek zu Stuttgart (1859), f. 39rv. In riferimento a questo testimone un passaggio del testo di Mattarelli viene segnalato da RYan, Political Thought, p. 432. Una moderna descrizione del codice di Tubinga, proveniente dall'Italia settentrionale e databile al secolo XV, in BrinKHUS-MenTZEL-Reuters, Handschriftenkatalog der Universitätsbibliothek Tübingen, p. 188 s. Riportando alcune opere attribuite al maestro lodigiano Martino Garato, il manoscritto è oggetto di studi specifici e di edizioni parziali del contenuto da parte di BAUMGärTNER, Martinus Garatus Laudensis, passim; EAD., «De privilegiis doctorum», pp. 312 ss., 323 ss.; EAD., An expediat habere multos libros, pp. 307 s., 322. Non viene segnalato in Murano, Martino Garati da Lodi.

60 WaelKens, La théorie de la coutume, pp. 119 ss., 161 ss.; FeEnSTRA-DuYnSTEE, Un recueil de textes de l'école de droit d'Orléans, p. 98. Una repetitio sul medesimo argomento viene attribuita, in forma dubitativa, anche a Jean de Monchy, maestro a Orléans di Jacques de Révigny: Bezemer, Les répétitions de Jacques de Révigny, pp. 261 s., 301. Altre leggi sulle quali si appunta l'attenzione dei giuristi nello sviluppare la riflessione sulla consuetudine sono quelle riunite nel breve Titolo Quae sit longa consuetudo (C. 8.52).

61 WaelKens, La théorie de la coutume, p. 250, nota 18.

62 Alberico la utilizza nel commentario alla 1. de quibus (Commentaria ad D. 1.3.31[32], f. 35rb, n. 60; cfr. WAELKENS, La théorie de la coutume, p. 142, nota 9: per un errore del copista l'allegazione viene attribuita a Jacques de Révigny) e filtra pure nella successiva opera di Bartolo.

63 Per le allegazioni di Mattarelli da parte di Cino (Cino Da Pistoia, Lectura super Codice, lib. VIII, tit. LIII Quae sit longa consuetudo, f. 519vb, n. 9 (ad 
linea di condivisione, nella definizione di consuetudine, dell'orientamento espresso in forma consolidata dai maestri bolognesi. Ne afferma anzitutto il carattere distintivo e precipuo di ius non scriptum ${ }^{64}$, in ciò ponendosi lungo la linea interpretativa che dopo Azzone ${ }^{65}$ era stata ripresa da Odofredo ${ }^{66}$ e che Cino fa propria rifacendosi anche alla lezione di Pierre de Belleperche ${ }^{67}$. La consuetudine, che tuttavia non trova nella scrittura l'elemento che la distingue prioritariamente dalla lex e che quindi può essere dichiarata sia in forma scritta che non ${ }^{68}$, trae forza dalla sua persistenza nel tempo e un intervallo di 10 anni è giudicato sufficiente affinché consuetudo dicitur lon$g a$, in quanto osservata per un diuturnum tempus che presuppone la voluntas di mantenerla realmente in vita ${ }^{69}$. La consuetudine, inol-

C. 8.53[52].1); f. 520rb, n. 1; f. 521ra, n. 4; f. 522rb, n. 9 in fi.; f. 522va, n. 11; f. 524rb, n. 21 (ad C. 8.53[52].2), e da Angelo degli Ubaldi nella repetitio manoscritta ad Inst. 1.2.9, si veda CORTESE, La norma giuridica, II, p. 106, nota 14 e p. 166, nota 146. Rassegna aggiornata dei manoscritti contenenti opere del più giovane fratello di Baldo in WoelKI, Angelo di Francesco degli Ubaldi, in part. p. 124 per il codice Ottoboniano latino 1726 contenente ai ff. $28 \mathrm{r}-35 \mathrm{v}$ la citata repetitio sulle Istituzioni e pp. 123 s., 128 per altre repetitiones e consilia di Angelo copiati nel codice Vat. lat. 5773, che conserva un esemplare del Tractatus super instrumentis di Mattarelli, per il quale si veda sotto, par. 8. È possibile individuare i passi relativi all'istituto dell'errore in rapporto alla consuetudine, allegati dai due commentatori, all'interno della repetitio-tractatus di Mattarelli, laddove si distingue tra error facti qui non habet in se consensus [populi], ut d. l. si per errorem (D.2.1.15) ed error iuris [...] qui non nocet cum sit error populi vel decurionum vel magistratum e in proposito si introduce l'ulteriore distinctio tra ius naturale e ius positivum (Stuttgart, Jur. fol. 123, f. 16ra-b).

64 «[...] consuetudo duobus modis dicitur. Uno modo dicitur consuetudo ius non scriptum, secundo dicitur comunis observantia que magistraliter et doctrine causa nominatur consuetudo» (Stuttgart, Jur. fol. 123, f. 12vb).

65 Azzone, Summa super codicem, p. 324b (ad C. 8.52); passo ricordato da WAELKENS, La théorie de la coutume, p. $248 \mathrm{~s}$.

66 OdofRedi, Lectura super Digesto veteri, lib. I, De legibus et senatisconsultis, f. 14vb, n. 2; f. 15rb, n. 6; f. 15vb, n. 12 (ad D. 1.3.32).

67 Cino da PIstoia, Lectura super Codice, lib. VIII, tit. LIII Quae sit longa consuetudo, f. 520va, n. 3 (ad C. 8.53[52].2); si veda anche CORTESE, La norma giuridica, II, p. 358, nota 117.

68 Stuttgart, Jur. fol. 123, f. 15va: «Circa consuetudinem declaratio fit duobus modis, sive expresse per scritpuram, et tunc est ius scriptum, aut per actus ordinatos cum continuitate temporis et tunc est ius non scriptum de quo tractamus in dictis legibus, ut C. de prescriptione longi temporis (C. 7.33)». Sulla scrittura come elemento non discretivo tra legge e consuetudine: CORTESE, La norma giuridica, II, p. 355 ss.

69 Stuttgart, Jur. fol. 123, f. 15ra: nella definizione di longa consuetudo Mattarelli richiama la concorde riflessione del dominus Io. [Giovanni Bassiano] et do- 
tre, debet esse ius generale e perciò non ammette un vincolo temporale differenziato tra presentes et absentes, per quanto Azzone, in ciò confortato dalla Glossa, oltre a rigettare l'idea di riconoscere la consuetudine soltanto se immemorabile (ex longevo tempore, cuius non extat memoria), avesse puntualizzato che un periodo di dieci o vent'anni sarebbe stato comunque sufficiente per poterla qualificare come longa $a^{70}$ : omnes tenet [consuetudo] tam maiores quam minores, tam absentes quam presentes sicut et leges scripte ${ }^{71}$. Un principio, anche questo, nella sostanza non certo nuovo ${ }^{72}$.

Nel solco autorevole ed esplicito di Govanni Bassiano e di Azzone si pone ancora Mattarelli fissando i presupposti in grado di formare una consuetudine, individuati nella semplice riproduzione di un atto in momenti diversi. Sine aliqua reiteratione, ciò è ritenuto sufficiente ad ius constituendum, ovvero a fissare un diritto nella cui spontanea formazione si ravvisa la tacita volontà del populus di preservarlo quale ius non scriptum sive consuetudo ${ }^{73}$.

minus Azo. Al Bassiano risale la tesi che a fondare una longa consuetudo bastassero dieci o vent'anni: CORTESE, La norma giuridica, II, p. $144 \mathrm{~s}$. L'orizzonte di dieci anni per fissare una longa consuetudo è condiviso da successivi maestri quali CINO (Lectura super codice, lib. VIII, tit. LIII Quae sit longa consuetudo, f. 523rb, n. 15 (ad C. 8.53[52].2), Alberico da Rosate (Commentaria ad D. 1.3.31[32], f. 36vab, n. 83) e IACOPO BOTTRIGARI (In primam et secundam veteris digesti partem, lib. I, tit. III De legibus et senatusconsultis, p. 13a, n. 8 (ad D. 1.3.32).

70 Azzone, Lectura [...] Codicis Iustiniani, lib. VIII, tit. LIII Quae sit longa consuetudo, p. 671, n. 1 (ad C. 8.53[52].2); gl. ad C. 8.53[52] in rub.: «[...] si ergo inducitur decem annis (ut dictum est) multo magis.XX. aut.XXX. annis».

71 Stuttgart, Jur. fol. 123, f. 15rb-va. Nell'affermare la validità uniforme della consuetudine Mattarelli si rifà esplicitamente a Odofredo e Iacopo Balduini, come già osserva WAELKENS, La théorie de la coutume, p. $250 \mathrm{~s}$.

${ }_{72} \mathrm{Si}$ veda ad esempio OdofREDI, Lectura super Digesto veteri, lib. I, De legibus et senatisconsultis, f. 16ra, n. 13: «[...] unde dicam quod consuetudo inducitur decennio et dicam quod ligat tam presentes quam absentes».

73 Stuttgart, Jur. fol. 123, f. 13va: «[...] et dominus Io(hannis) et Azo dicunt quod duplex talis actus interveniens et tempus diversitate sit certus inducet consuetudinem, quia unus actus facit consuetudinem, ut C. de episcopali audientia, 1..iii. in fi. (C. 1.4.3.4). [...] si fuerit talis actus duplicatus et diversitate temporis comprobatus possit presumi et intelligi per talem modum populum sic voluisse pro iure servari et sic erit ius non scriptum sive consuetudo». Sul punto, la diversa linea interpretativa sostenuta da Iacopo Balduini e sviluppata in seguito dai maestri orleanesi, che reclamava la ripetizione più frequente di un atto per configurare la consuetudine e affidava piuttoso al magistrato la valutazione del numero di atti necessari alla sua precisa individuazione, viene esaminata da WAELKENS, La théorie de la cou- 
Ancora nell'ambito della produzione di repetitiones da parte di Mattarelli va ricordato un commento alla legge Cum pro eo (C. 7.47. un.) presente all'interno di una cospicua raccolta di Consilia et allegationes quasi sicuramente approntata in ambiente modenese nei primi decenni del secolo XVI benché a noi giunta in forma certamente rimaneggiata ${ }^{74}$. La repetitio, sviluppata su una nota costituzione giustinianea del 531 in tema di protezione dei debitori ${ }^{75}$, nel codice viene affiancata a quella attribuita, sul medesimo argomento, a Pierre

tume, p. 262 ss. Già CoRTESE, La norma giuridica, II, p. 144 ss., poneva l'accento sul confronto tra una linea interpretativa orientata a individuare «una componente essenziale del processo creativo della consuetudine» nel «decorso del tempo combinato con una determinata prassi giudiziaria» (p. 146) e un'altra, già ravvisabile fin dagli esordi della Scuola bolognese, incline piuttosto a svalutare questi due elementi rispetto all'egemonia della voluntas populi.

${ }_{74}$ Modena, Biblioteca Estense, ms. lat. 1161, ff. 133r-161r: inc. Nicolai Mattarelli mutinensis repetitio eiudem lex unica de sententiis, que pro eo quod interest; expl. Ideo quia plenius ac clarius posui super hanc legem in hanc scripturam reducere ac compilare disposui. Ego Nicolaus de Mattarellis mutinensis (cartulazione moderna in cifre arabe a matita nell'angolo inferiore sinistro del recto di ciascuna carta). Si tratta di un codice miscellaneo di estremo interesse, composto da fascicoli di dimensioni e grafie diverse; quelli centrali contengono pareri e consilia dotati anche di sigillo finale e di firma autografa; tra i più significativi quelli di Vincenzo de Paliottis bolognese, Bartolomeo Socini da Siena, Francesco Tigrini da Pisa, Angelo degli Ubaldi, Cristoforo Castiglioni, Giovanni Petrucci da Montesperello, Floriano di San Pietro, Giovanni Sadoleto modenese, Giovanni da Imola (Padovani, Dall'alba al crepuscolo del commento, p. 100), Carlo Ruini e Francesco de Pepis. Per grafia e dimensioni si può supporre che i fascicoli A-M, ff. 17r-172v, terminanti con un parere in materia di dote rilasciato da Francesco Guicciardini, commissario pontificio di Modena dal 1516 al 1524 (ff. 169r-172r), siano parte della raccolta cinquecentesca originaria, alla quale furono aggregati altri fascicoli contenenti consilia di autori vari; opera di assemblaggio che non pare di molto successiva, considerata la grafia cinque-seicentesca sia della primitiva numerazione, in cifre arabe a penna poste nell'angolo superiore destro di ciascuna carta, sia degli indici iniziali di autori e materie, premessi al restante materiale e privi di numerazione delle rispettive carte. L'intitolazione di Consilia et allegationes si legge a f. 1r. Una limitata e parziale descrizione del codice alla URL $<$ http://manus.iccu.sbn.it//opac_Scheda $\% 20$ Scheda.php? ID $=216660>$.

75 La costituzione, non limitandosi a fissare il tetto degli interessi, investe l'ammontare del debito nella sua globalità stabilendo che, a prescindere dalle ragioni addotte dal creditore, il debitore non possa essere condannato a risarcire più del doppio del debito originario in tutti i casi in cui quest'ultimo sia accertabile, diversamente si raccomanda moderazione ai giudici facendo affidamento sulla loro subtilitas: in merito si rinvia a TAFARO, C.I. 7.47.1. Giustiniano e i limiti della condanna del debitore, p. 226 ss.; ID., Debito e responsabilità. Profili romanistici, p. 44 ss.; ID., La limitazione dei debiti. Sul tema specifico, l'orientamento della dottrina 
de Belleperche, tenuta dal maestro ultramontano a Bologna durante il viaggio a Roma in occasione del primo giubileo proclamato da papa Bonifacio VIII ${ }^{76}$. Si tratta probabilmente di una copia tratta da una reportatio della lezione tenuta dal giurista francese e ritenuta interessante per essere inserita nella raccolta modenese, considerata la rinnovata attenzione per l'opera di Belleperche favorita dall'attività didattica svolta da Jean Feu a Pavia negli anni 1509-11 e dalla pubblicazione sempre a Pavia, nel 1513, di alcune sue repetitiones ${ }^{77}$.

Il fatto di rintracciare la repetitio di Pierre de Belleperche accanto a quella sviluppata da Mattarelli sulla medesima legge del Codice non sembra dovuto a interessi comuni o convergenze dottrinarie tra i due doctores, dal momento che quest'ultimo non mostra mai di confrontarsi con le opzioni interpretative dei maestri ultramontani e ne ignora le opere, che entrano progressivamente nell'orizzonte dei giuristi italiani - come ben noto - a partire dalla generazione di Cino e Giovanni d'Andrea, e non sempre per conoscenza diretta e citazioni di prima mano $^{78}$. Denominatore comune

giuridica nell'età dei Glossatori è oggetto di recenti indagini da parte di CONDORELLI, Sul contributo dei canonisti alla definizione del concetto di interesse.

76 Modena, Biblioteca Estense, ms. lat. 1161, ff. 118r-132v: inc. Petri de Bellapertica de anno domini m.ccc. Bononiae. Repetitio lex unica. De sententiis quae pro eo quod interest (C. 7.47.un.); expl. Animal irrationale contra propria semper pugnans utilitate. Come noto, nella città felsinea la repetitio tenuta da Belleperche sarebbe stata ascoltata personalmente da Cino, che la ricorda nel commento alla medesima legge del Codice: CINO DA PISTOIA, Lectura super Codice, in lib. VII, tit. XLVII De sententiis, f. 458va, n. 6 in fine; Cortese, Il diritto nella storia medievale, II, p. 411.

77 Soetermeer, Recherches sur Franciscus Accursii, p. 25 ss. e p. 43 s. per la parziale edizione della repetitio di Pierre de Belleperche. Su Jean Feu (Joannes Ignaeus, 1477, o più probabilmente 1483/84-1549) e la sua attività pavese si vedano Cortese, Controversie medievali sul dominio utile, p. 617 ss., e ID., Jean Feu a Pavia: da parte del giurista transalpino il richiamo a Pierre de Belleperche, più volte espresso nel corso di una repetitio solenne tenuta presso lo Studium pavese il 10 agosto 1509 , funge soprattutto da «vessillo simbolico di una scienza francese brillante scatenata al contrattacco di un bartolismo italiano che viene gabellato tutto per filoimperiale e retrogrado» (p. 133). Si ricorda pure il succinto profilo biografico di Arabeyre, Feu (Ignaeus) Jean. Repetiones del giurista orleanese sono raccolte nel volume Index operum d. Johannis Ignei doctoris [...] \& primo eiusdem solemnis \& valde quotidiana repetitio L dudum L. de contrahen. emptione. Eiusdem pomposa \& elegantissima repetitio auctentice ex causa. L. de libe. [...], Papiae, per magistrum Bernardinum de Garaldis, 1513.

78 MeIJERS, Etudes d'histoire du droit, III, p. 114 ss. 
ai due testi è indubbiamente lo stesso Cino, che mostra di utilizzarli entrambi nel proprio commento alla legge Cum pro $e o^{79}$, ma ciò non aiuta a illuminare il criterio seguito dal compilatore cinquecentesco nel riunire assieme i testi cronologicamente più risalenti dell'intera raccolta.

Nel codice modenese le due repetitiones sono riprodotte all'interno di un gruppo di 4 fascicoli ${ }^{80}$, precedute da una copia del trattatello di Iacopo d'Arena De praeceptis iudicum ${ }^{81}$ e seguite da materiale di ambito strettamente locale consistente in un casus relati-

79 SoEtermeER, Recherches sur Franciscus Accursii, in part. p. 27 s. per la conoscenza del testo della repetitio in seguito confluito nel codice modenese. Per i rinvii alla repetitio di Mattarelli ad C. 7.47.un. si veda CINO DA PIsToIA, Lectura super Codice, in lib. VII, tit. XLVII De sententiis, f. 460vb (Nicolaus vero Matharellus mutinensis doctor, qui longo tempore rexit in studio paduano, dicit quod certi casus et contractus sunt illi, in quibus non petitur interesse iure actionis a principio debiti. Incerti vero sunt, ubi interesse a principio iure actionis venit), f. $461 \mathrm{vb}$ e f. 462ra, ove è richiamata due volte e approvata l'opinione di Mattarelli nello sviluppo della penultima quaestio che scandisce il commento ciniano (qualiter interesse deductum in iudicium probetur?) in merito alle modalità di prova in giudizio dell'interesse vantato dal creditore. A margine si può osservare che la Lectura Codicis viene terminata da Cino l'11 giugno 1314 (Maffer, Cino Sinibuldi da Pistoia, p. 544), lo stesso mese ed anno in cui Mattarelli è ricordato come già defunto all'interno del testamento del figlio Francesco.

80 Fascicoli di uguale formato distinti dalle lettere $\mathrm{H}$ (ff. 107r-120v), I (ff. 121r-136v), K (ff. 137r-152v), L (ff. 153r-168v). Tutti i fascicoli precedenti A-G (ff. 1r-106v) contengono consilia e allegationes di dottori e giureconsulti toscani, in larga parte fiorentini; materia analoga caratterizza pure i fascicoli successivi, ove però si osserva una maggiore varietà nell'origine degli autori, come il reggiano Carlo Ruini (1456-1530) e il senese Bartolomeo Socini (1436-1507).

81 Modena, Biblioteca Estense, ms. lat. 1161, ff. 107r-117r: inc. Cum queritur de preceptis factis in suos subditos continentibus aliquid dari vel fieri, ut plenius de talibus preceptis notitia habeatur, sic distinguendum est; expl. Recipienda est igitur intentio, quia continet id, quo probato, iustitia et veritas iam facti precepti in aperto clarebit. Finis. Si tratta di una versione compendiata, in numerosi passaggi, del trattatello stampato in TUI, III/II, Venetiis 1584, ff. 34vb-35vb. L'esemplare modenese è privo dell'ultimo paragrafo leggibile nell'edizione a stampa che si chiude con una allegazione di Cino, unica in tutta l'opera, in base alla quale MARCELLo, Opere giustamente e ingiustamente attribuite a Iacopo d'Arena, p. 856, ne respinge l'attribuzione a Iacopo. Pare tuttavia che quest'ultimo paragrafo, dedicato al tema An a preceptis iudicum possit appellari ma assente dall'indice iniziale del trattato (nn. 1-23), sia frutto di un'aggiunta posteriore non confluita nell'esemplare utilizzato per la copia modenese. Un esemplare incompleto del medesimo trattato anche nel codice Bologna, Collegio di Spagna 122, ff. 348v-349v (corrispondente alla edizione in TUI, III/II, ff. 34vb-35rb, nn. 1-16), che si aggiunge agli altri due manoscritti censiti in Quagloni, Dell'Arena (D’Arena) Iacopo, p. 248. 
vo a una controversia, datata 1525 , tra il Giudice delle vettovaglie - la magistratura civica che sovrintendeva ai vari settori dell'economia urbana - e i titolari di alcune botteghe di calzolai ${ }^{82}$. Nell'ottica del compilatore cinquecentesco della vasta miscellanea giuridica la scrittura di questo manipolo di fascicoli sembra prescindere da un criterio di omogeneità tematica; soltanto le due repetitiones di Pierre de Belleperche e Mattarelli sono dedicate al medesimo argomento e riprodotte l'una di seguito all'altra, e la disponibilità di un testo riferibile al noto giurista modenese - l'unico conservato nella sua città di origine - può avere suggerito di copiarne un altro, immediatamente accanto, ispirato a un caso giuridico scaturito da una situazione di ambito strettamente municipale.

5. Nello strumentario del giurista postaccursiano: le quaestiones

Nell'ambiente padovano, rappresentato da ben noti allievi di Oldrado quali Guglielmo da Pastrengo e Alberico da Rosciate e da prestigiosi docenti del calibro di Giovanni D’Andrea, la produzione dottrinaria di Mattarelli - come già osservato in precedenza - viene legata soprattutto alla redazione di quaestiones e repetitiones ${ }^{83}$.

82 Modena, Biblioteca Estense, ms. lat. 1161, ff. 161v-165v: la controversia scaturisce dalla multa comminata dallo Iudex victualium civitatis Mutine, che agisce vigore potestatis sibi attribute, ad alcuni sutores, qui in die prohibito apote(c)has tenebant apertas, nonostante la deroga al divieto prevista dagli statuti dell'Arte. Il Casus è datato Mutinae 1525 e sottoscritto da Petrus Benintendus Cesenas iud. Da rilevare che il successivo fascicolo M (ff. 169r-172v) contiene un parere in materia di dote rilasciato da Francesco Guicciardini negli anni in cui è governatore di Modena e Reggio (1516-24). Inc. Divino nomine implorato. In presenti consultatione breviter me expediam tum quia res iuditio meo est satis clara, tum quia plurimis occupationibus ita sum implicitus ut cogar brevis esse; expl. Ex quibus concludo parvus D. U. esse in pronuntiando iudices mercantie esse incompetentes et non posse procedere super dicta causa, et ita consulo et arbitror. Ego Franciscus de Guicciardinis I. U. doctor Mutine et Regii gubernator.

83 Quaestiones di Mattarelli sono ricordate anche come disputationes sia da Giovanni D'Andrea, In secundum Decretalium librum Novella commentaria, de fide instrumentorum, f. 172v, n. 20 (in X.2.22.10), sia da Alberico DA RosciaTE, Commentaria, de in litem iurando, 1. nummis depositis (D. 12.3.3), f. 38ra, n. 3 in fi. Una disputatio di Mattarelli sulla l. Cunctos populos (C. 1.1.1) è ricordata all'interno di una additio posta a margine del Digesto nuovo in corrispondenza 
Grazie anche alla preziosa testimonianza di Alberico e Giovanni, che di frequente si dimostrano «attenti a segnalare sequele di opinioni quando su un tema eguale o similare erano note due o più quaestiones di predecessori o contemporanei ${ }^{84}$, il duttile genere della quaestio, più o meno disputata, emerge indubbiamente come quello ove il maestro modenese ha lasciato considerevoli e prevalenti testimonianze. Ed è anche rilevante notare come la copiosa raccolta posseduta da Alberico e qualificabile come un vero e proprio Liber magnus quaestionum disputatarum, una delle tre antologie di questo tipo a sua disposizione, si aprisse con una quaestio di Mattarelli in materia di bando e altre ne seguissero nelle carte successive ${ }^{85}$.

Dalle allegazioni e citazioni emergenti dalle opere dei due giuristi affiora il profilo di un Mattarelli interessato a una pluralità di tematiche fortemente imperniate sul mondo delle istituzioni e della

della 1. Interdum (D. 39.4.16) nel ms. La Seu d'Urgell 2031, f. 18r: Legendre, Manuscrits avec gloses postaccursiennes, p. $702 \mathrm{~s}$. Per una descrizione dapprima sintetica e quindi maggiormente analitica di tale codice si rinvia a BERTRAM-MafFEI, $A$ Catalogue of the Manuscripts in the Chapter Library of La Seu d'Urgell, p. 67, e al successivo Catálogo de los manuscritos jurícos de la biblioteca capitular de la Seu d'Urgell, pp. 56-59.

84 Bellomo, Quaestiones in iure civili disputatae, p. XIII, nota 27.

85 Quaestio «Quid sit bannum et unde dicatur» in Alberico DA Rosciate, Commentarium de Statutis, 1. IV, q. 1, f. 65vb, n. 1. La prima quaestio all'interno del liber magnus in possesso di Alberico viene ricordata anche ibidem, 1. III, q. 2, f. 55va, in fi., con tuttavia riferimento alla quaestio «Quidam inculpatus de homicidio». Altre quaestiones affrontate da Mattarelli sono ricordate da Alberico all'interno della propria raccolta distinte dai numeri 299, 302 e 305: ibidem, l. I, q. 150, f. $24 \mathrm{rb}$, n. 17; 1. II, q. 32, f. 33vb, in.; 1. II, q. 44, f. 35v, n. 16. Una ulteriore quaestio di Mattarelli, dedicata al tema dell'imperium e del titolo che ne legittima l'esercizio, viene ricordata da Alberico come una disputatio e collocata al terzo posto nel liber magnus a sua disposizione: Alberico da Rosciate, Commentaria, de iurisdictione, 1. imperium (D. 2.1.3), f. 93rb-va, nn. 41-45 e ibidem, de iurisdictione omnium iudicum, 1. perinicuum (C. 3.13.7), f. 153ra-b, n. 12. Si veda anche sotto, nota 79. Alcune citazioni già in Solmi, Alberto da Gandino, p. 170 s., nota 2; si veda più ampiamente Romano, Le quaestiones disputatae, p. 63 ss. e n. 37, p. 95 s.; n. 48 , p. $101 ;$ n. 103 , p. $134 ;$ n. 128 , p. 149. Considerazioni sulla ricostruzione della struttura del perduto esemplare di liber magnus a disposizione di Alberico si devono a Bellomo, I fatti e il diritto, p. 141 ss. Altri esemplari di raccolte in possesso del giurista bergamasco sono ricordate come Quaestiones Bononiae disputatae e Quaestiones per doctores antiquos Bononiae disputatae, e anche Giovanni d'Andrea ne possedeva una, ma diversa per assortimento e distribuzione interna dei testi: ibidem, pp. 145 s., 547, e già Miglionino, «Dominus meus in legibus», p. 124 e nota 15. 
società comunale, con spiccato e prevalente interesse verso l'ambito giudiziario, le problematiche di tipo successorio, le prescrizioni della normativa statutaria, le modalità di redazione e il valore probatorio delle scritture notarili. Non sembra invece documentata l'attenzione specifica di Mattarelli verso temi che toccano orizzonti di respiro più ampio rispetto a quelli abbracciati dagli ordinamenti locali, come i fondamenti dell'assetto politico-giuridico di quella potestà temporale che ancora al suo tempo tenta di rinnovare progetti di egemonia sulla penisola italiana. Egli non compare, infatti, tra i civilisti italiani e francesi alle cui opere lo stesso Alberico attinge, avendo anche conoscenza diretta degli autori transalpini, nell'elaborazione di una dottrina sui poteri dell'impero incentrata soprattutto sulla valutazione della Donazione di Costantino, che egli giunge a considerare invalida sulla base della indisponibilità e imprescrittibilità degli iura imperii e della iurisdictio imperiale ${ }^{86}$. Dal punto di vista speculativo Mattarelli non fornisce un contributo significativo su questo livello di argomenti, preferendo concentrarsi su ambiti più aderenti alla vita degli ordinamenti particolari e al raccordo tra normativa locale e sistema generale delle leges. Ne è un esempio una quaestio sviluppata in tema di imperium riferito all'ambito circoscritto della giurisdizione applicata su un nucleo signorile castrense, nella quale la problematica più generale si confronta con il mondo delle vive relazioni basate sulle situazioni di fatto. Un caso da cui il giurista prende spunto per esaminare i profili concreti rispetto ai quali misurare la legittimità del potere rivendicato in iudicio dal quidam nobilis distinguendo, nella solutio conclusiva, le fattispecie che si possono considerare per confermare o respingere le pretese dell'attore e che si allineano entro il preciso spazio delimitato, per un versante, dalla concessione e dall'autorità del princeps e, per l'altro, dalla concessione e dal consenso del populus ${ }^{87}$.

86 Conetti, La dottrina dell'impero, p. 324 ss.

87 «Quidam nobilis contendit in iudicio imperium, iurisdictionem et omnem potestatem ad se pertinere in quodam castro»: ASP A.29, ff. 284rb-285va; edizione in Bellomo, Le istituzioni particolari e i problemi del potere, p. 235 ss., con ulteriori riferimenti e commento a p. 212 ss. e in ID., I fatti e il diritto, p. 259. La quaestio di Mattarelli è nota sia ad Alberico da Rosciate, da lui collocata al terzo posto all'interno di un suo liber magnus (vedi sopra nota 71), sia a Baldo (vedi sotto cap. III, nota 29). 
Nell'ambito della vastissima casistica desunta dal funzionamento della giustizia pubblica comunale, di Mattarelli si ricordano quaestiones attinenti a profili sia procedurali ${ }^{88}$ sia sostanziali che investono situazioni di rilievo penale ${ }^{89}$, accanto alla produzione e alla

88 Quaestio disputata Paduae «Si iudex assumpsit tres consultores super diffinitiva, quorum duo consulerunt reum condemnandum, tertius absolvendum, et iudex absoluit, an valeat sententia, cum statutum habeat non valere sententias, nisi latae sint cum consilio sapientium», che Mattarelli risolve riconoscendo comunque validità alla sentenza in quanto pronunciata nel rispetto dello statuto che richiedeva unicamente il preventivo ricorso al consilium sapientis; nel caso poi che essa fosse giudicata iniqua, non rimaneva che avvalersi dei communia remedia, ovvero il ricorso in appello (GIOvanNI D'ANDREA, In titulus de Regulis iuris, in reg. 62.VI, f. 54ra-va; richiamata pure in Guglielmo Durante, Speculum iudiciale, II, II, de requisitione consilii, add. consilium, t. 1, p. 763). La quaestio è analoga, nella sostanza, a quella con inc. (Sequitur.vii. quaestio domini Nicolai Ma.) Statutum est in civitate Padue quod quilibet iudex teneatur sententias ferre cum conscilio sapientum non suspectorum et alia sententia lata non valeat; expl. sententia inter eos lata, alia non, ut ff. de pignoribus, 1. si superatus (D. 20.1.3) et ibi notatur et ff. de exceptione rei iudicatae, 1..i. (D. 44.2.1) secundum dominum Nico. de Ma. de Mutina, leggibile in ASP A.29, ff. 280va-281rb, e in Collegio di Spagna 81, ff. 83r-84r (ff. 82r-83r cartulazione antica); si veda Bellomo, I fatti e il diritto, p. 378; ID., Quaestiones in iure civili disputatae, n. 356, p. 184; MAFFEI, I codici del Collegio di Spagna, p. 168. Quaestio «Quidam veniens ad penitentiam confessus fuit» (Guglielmo Durante, Speculum iudiciale, II, III, de appellationibus, add. confessus, t. 1, p. 831, richiamata pure ibidem, IV, IV, de raptoribus, incendiariis et violatoribus ecclesiarum, add. in rubrica, t. 2, p. 493), leggibile in ASP A.29, ff. 287ra-288rb e Chigi E.VIII.245, ff. 129vb-130va; si veda Bellomo, I fatti e il diritto, p. 189, nota 121; ID., Quaestiones in iure civili disputatae, n. 362, p. 186 s. e n. 183 , p. 293. Quaestio disputata «de illo, qui per stipulationem ad id tenebatur, condemnato per potestatem ad equum restituendum Titio intra xxx dies sub poena decem librarum solvendarum communi; termino transacto potestas pro communi vult poenam, actor petit illum condemnari quantum in litem iuraverit; quaeritur an procedant praedicta duo, an neutrum, an alterum solum et quod. Et incipit quaestio Quaedam per Potestatem Paduae» (Guglielmo Durante, Speculum iudiciale, IV, II, de iureiurando, add. actione, t. 2, p. 182). In merito Sorrenti, Testimonianze di Giovanni D’Andrea, n. 54, p. 60 s. e n. 123, p. 84; n. 145, p. 92 e n. 345, p. 161; n. 212, p. 119.

89 Quaestio olim fuerat disputata [...] in studio paduano «De illo qui occidit permissione iudicis vel statuti clericum bannitum, qui laicus putabatur, an excusetur a dolo et poena» (GIOVANNI D'ANDREA, In titulus de Regulis iuris, in reg. 24.VI, f. 81rb). Quaestio "An veniret Titius condemnandus de occiso, contra quem duos testes deposuerunt, quod notorium est, quod occidit Seium» (Guglielmo Durante, Speculum iudiciale, III, I, de notoriis criminibus, add. ut nostrum, t. 2, p. 52). In merito Sorrenti, Testimonianze di Giovanni D'Andrea, n. 182, p. 108. Si aggiunga la quaestio «Commisso quodam homicidio in conspectu potestatis pro tribunali sedentis potestas per inquisitionem contra quendam processit et aliquibus inditiis 
valutazione in giudizio di publica instrumenta ${ }^{90}$ e alla riflessione sul tema della pace privata, sviluppata a integrazione di una quaestio attribuita a Nicola da Cremona ${ }^{91}$. La materia civilistica è orienta-

motus tormentavit eundem, qui confessus fuit de maleficio» in ASP A.29, ff. 264rb265vb; si veda Bellomo, Quaestiones in iure civili disputatae, n. 344, p. 177.

90 Con riferimento alla quaestio «Titius coram iudice», per cui si veda sotto, nota 80 , e alla quaestio prolixa et de facto «Actor produxit instrumentum confectum secundum scripturam, nunc sunt XX anni [...]» (Guglielmo Durante, Speculum iudiciale, I, IV, de teste, add. § fi., t. 1, p. 320), identificabile con quella con incipit «In quadam lite que erat occasione cuiusdam instrumenti scripti modo sunt. XXIX. anni contra ipsum instrumentum producti et publicati sunt testes [...]», leggibile in ASP A.29, ff. 283ra-284rb; si veda SorrenTI, Testimonianze di Giovanni D'Andrea, n. 51, p. 59; Bellomo, Quaestiones in iure civili disputatae, n. 359, p. 185. Quest'ultima trova corrispondenza nella quaestio con incipit «In quadam questione quae erat occaxione cuiusdam instrumenti scripti modo sunt.XXIIII. anni contra ipsum instrumentum producti sunt et publicati testes [...]» leggibile nel ms. Foligno, Biblioteca Jacobilli, A.VI.17, ff. 32r-33v, che differisce tuttavia nella parte finale chiusa dal richiamo al parere del maestro: «Et ita consulo ego Nicolaus de Mattarelli». Per la descrizione complessiva del codice si rinvia a Sorbelli, Inventari dei manoscritti delle biblioteche d'Italia, n. 123, pp. 57-59: i ff. 1-35 contengono consulti legali di numerosi giuristi che, con limitate varianti, si chiudono tutti con la formula ricorrente «Et ita consulo ego ...» Iacobus Buctrigarius (f. 1r), Baldus de Perusio (f. 1r), Marcus de Perusio (f. 2r), Baldus de Perusio (f. 12r), Iohannes de Lignano iuris utriusque doctor (f. 12v), Francischus de Albergottis (ff. 14v-20r), Baldus de Perusia (f. 21r), Federicus de Senis decretorum doctor (f. 21v), Franciscus de Albergottis de Aretio civis Florentinus legum doctor (f. 22v), Baldus de Peruxio iuris utriusque doctor (f. 22r), Iohannes de Lignano subscripsi et sigillavi (f. 22v), Baldus de Peruxio (f. 23r), Ricardus de Saliceto de Romania legum doctor (f. 23r), Franciscus de Albergottis de Aretio legum doctor (f. 24r), Franciscus de Albergottis (f. 25r), Baldus de Perusio (f. 26v), Iohannes Pagliarensis de Senis legum doctor (f. 27v), Anthonius de Aretio (f. 28r), Dyonisius de Barigianis de Perusio iuris utriusque doctor (f. 28v), Franciscus de Albergottis (f. 29v), Franciscus de Albergottis (f. 30r), Rainerius de Monterubiano legum doctor (f. 30v), Griffolus de Monte Palitiano legum doctor (f. 31r), Franciscus Tigrini (f. 31v), An(tonius) de Pii et Cinus de Pistorio legum doctores (f. 32r), Nicolaus de Mattarellis (f. 33v), Nicolaus de Mattarellis (f. 35r).

91 «Iohannes ex parte una, Martinus ex altera fecerunt inter se pacem» (Vat. Lat. 10726, f. 292r), censita in Borino, Codices Vaticani Latini. Codices 1070110875, p. 134, n. 200. La quaestio è trascritta anche in Chigi, E.VIII.245, f. 251rbva: si veda Bellomo, Giuristi cremonesi e scuole padovane, pp. 140, 146, con edizione parziale a p. 159 s.; ID., Factum proponitur certum, p. 3, nota 7; ID., I fatti e il diritto, p. 305 s.; ID., Quaestiones in iure civili disputatae, n. 395, p. 377 s. In entrambi i codici la quaestio di Nicola da Cremona è seguita da una additio di Mattarelli (con edizione in Bevilacoua, Nicolai de Mactarellis Consilium de pace promissa et non servata) vòlta ad approfondirne due profili preminenti tramite il ricorso a fonti sia romanistiche sia scritturali e anche a un passo dei libri feudorum. 


\section{ta, in particolare, verso problemi successorii ${ }^{92}$ o comunque inerenti la trasmissione ereditaria ${ }^{93}$, verso fattispecie riguardanti la materia}

92 «Quidam in testamento fratrem suum instituit heredem et rogavit eum ut certis personis certa legata daret»: in ASP A.29, ff. 271ra-272rb e in Collegio Spagna 81 , f. 86v (f. 85v cartulazione antica) (inc. Sequitur quinta quaestio dicti domini Nicolai. Quidam in testamento fratrem suum heredem instituit et rogavit eum ut certis personis certa legata daret; expl. sub aliqua specialitate relicta sunt ut in legibus ad hoc superius allegatis secundum dominum Nic.); si veda BelLomo, Quaestiones in iure civili disputatae, n. 349, p. 180 e MAfFeI, I codici del Collegio di Spagna, p. 169. "Quidam filios non habens fecit testamentum in quo talem fecit institutionem et substitutionem»: in ASP A.29, ff. 276ra-277va e in Chigi E.VIII.245, ff. $140 \mathrm{va}-141 \mathrm{rb}$; edizione parziale in BeLlomo, Giuristi cremonesi e scuole padovane, p. 161 s., e p. 142, nota 23; si veda anche ID., I fatti e il diritto, pp. 192, nota 134, 298, 303; ID., Quaestiones in iure civili disputatae, n. 352, p. 181 s. e n. 203, p. 302; WeIJERS, Queritur utrum, p. 132 s. «Quidam fecit testamentum in quo constituit extraneum quendam eredem»: in ASP A.29, ff. 277va-278va e in Chigi E.VIII.245, f. 132rb-vb; si veda Bellomo, Legere, repetere, disputare, p. 42, nota 61; ID., Quaestiones in iure civili disputatae, n. 353, p. 182 e n. 186, p. 294. È utilizzata da Giovanni d'Andrea in Guglielmo Durante, Speculum iudiciale, II, II, de instrumentorum editione, add. penultimo, t. 1, p. 681, su cui SorrenTI, Testimonianze di Giovanni D'Andrea, n. 98, p. 76. «Quidam filius familias habens bona adventitia factus est furiosus et postea institutus est heres a patre cum quodam suo fratre germano»: ASP A.29, ff. 279rb-280va; si veda BeLLOMO, Quaestiones in iure civili disputatae, n. 355, p. 183. «Duos fratres a patre heredes instituti rogati fuerunt ut quicumque decederet sine liberis restitueret hereditatem superstantibus»: ASP A.29, ff. 282va-283ra, e Collegio di Spagna 81, f. 85r-v (f. 84r-v cartulazione antica) (inc. Undecima quaestio dicti domini Ni. Duos fratres a patre heredes instituti rogati fuerunt ut quicumque eorum decederet sine liberis; expl. et hec videtur mihi esse intentio legis in corpore de sanctis episcopis $\S$ Sed et haec praesenti (Nov. 123.37 = Auth. 134, Coll. IX, tit. 15) et secundum dominum Ni. de Ma.); si veda Bellomo, Quaestiones in iure civili disputatae, n. 358, p. 185 e MAfFeI, I codici del Collegio di Spagna, p. 168. «Quidam habens duos filios, unum ex primo matrimonio alterum ex secundo, primum emancipavit»: ASP A.29, f. 251va-b (edita parzialmente in Bellomo, Giuristi cremonesi e scuole padovane, p. 169); si veda ID., Quaestiones in iure civili disputatae, n. 396, p. 378. Si aggiunga la quaestio «Si probetur per testes quod testator dixit: Titius haeres est, et in scriptura testamenti continetur solum 'Titius' et abest ' haeres esto', an valebit?» ricordata da Baldo in Baldi Ubaldi, In sextum codicis librum commentaria, f. 61 va, n. 6 (ad C. 6.23.7).

93 «An, fideicommissario ex speciali potestate nominante alium fideicommissarium, iste secundus habeat eandem potestatem quam primus vel quam haberet directe factus a testatore» $\mathrm{e}$ «An ipse commissarius vivens possit alium subrogare vel per alium officium suum implere», utilizzate da Giovanni d'Andrea in Guglielmo Durante, Speculum iudiciale, II, II, de instrumentorum editione, add. nulli, t. 1, p. 715 s.; si veda SorrentI, Testimonianze di Giovanni D’Andrea, n. 104, p. 78. Sul tema delle obbligazioni contratte con minori si ricorda la quaestio «Statutum est in civitate quod nullus contractus et nulla obligatio fiat cum minore sine auctoritate tutoris et si contra factum fuerit non valeat ipso iure», trascritta in ASP A.29, ff. 
del prestito e del mutuo ${ }^{94}$ e verso il mondo degli scolari, strettamen-

281rb-282va e Collegio di Spagna 81, ff. 84r-85r (ff. 83r-84r cartulazione antica) (inc. Sequitur.x. questio dicti domini Nicolai quae talis est. Statutum est in civitate quod nullus contractus et nulla obligatio fiat cum minore sine auctoritate curatoris; expl. contractus nullus, non obstante autentica Sacramenta (post C. 2.27[28].1) ut supra in allegationibus pro. i $^{\text {a }}$ parte dictum est secundum Nico de Ma.); si veda BELLOMO, I fatti e il diritto, p. 378; ID., Quaestiones in iure civili disputatae, n. 357, p. 184 e MAfFer, I codici del Collegio di Spagna, p. 168.

94 «Titius coram iudice talem petitionem exposuit. Dicit Titius quod Seius fuit confessus se recepisse.C. libras in depositum ab eo, unde petit eum sibi condempnari ad restitutionem» (ASP A.29, ff. 272rb-273vb); si veda Bellomo, Quaestiones in iure civili disputatae, n. 350, p. 180 s. È utilizzata da Giovanni d'Andrea in Guglielmo Durante, Speculum iudiciale, I, IV. de teste, add. fundus, t. 1, p. 297 e ibidem, IV, III, de deposito, add. in rubr., t. 2, p. 224. Si veda Sorrenti, Testimonianze di Giovanni D'Andrea, n. 46, p. 57 e n. 222, p. 121. Già Colle, Storia scientifico-letteraria dello Studio di Padova, p. 81, notava come Giovanni d'Andrea ricordasse una disputa tenuta da Mattarelli «sulla questione di un deposito, ove si vede una eccessiva cavillosità e un sommo abuso dei termini e delle formalità legali, onde liberare giudiziariamente un debitore dal restituire un deposito che aveva per propria confessione ricevuto». La quaestio è trascritta anche in Archiginnasio B.1393, ff. 95v-97r: «An confessio depositi sufficiat ad agendum in probato deposito. Rubrica». Sul codice miscellaneo bolognese, contenente anche quaestiones disputatae di maestri attivi nello studio patavino come Pietro d'Ancarano e Angelo degli Ubaldi, si veda Mazzatinti, Inventari, 79, p. 23 s.; Lucchesi, Manoscritti della Biblioteca Comunale dell'Archiginnasio, p. $38 \mathrm{~s}$. Il codice, giunto in proprietà del notaio vescovile bolognese Rolando Castellani, era appartenuto a Luca Cantarelli da Reggio, addottoratosi a Padova in diritto canonico nel 1399 e allievo di Francesco Zabarella, in seguito vicario del vescovo di Bologna Nicolò Albergati nel periodo 1419-22 e ancora presente a Bologna negli anni 1437-38: si veda la voce redazionale Cantarelli Luca, in DBI, 18, Roma 1975, pp. 239-240, integrata da PARMEGgiani, Visite pastorali e riforma a Bologna, p. 37, nota 59. A Mercati, Per la storia letteraria di Reggio Emilia, p. 65 ss., si deve una rassegna di codici appartenuti al Cantarelli e per gran parte acquisiti in seguito dalla Bodleian Library di Oxford, dalla quale è tuttavia assente il codice bolognese sopra citato. Secondo la nota di possesso leggibile a f. 146r, il notaio Castellani acquistò il codice dal converso certosino Giovanni Bitini, esecutore, in qualità di amministratore dei Pauperes Christi di Bologna, di un codicillo testamentario di Luca Cantarelli. Altre quaestiones in materia di prestito e di mutuo: «Quidam per potestatem Padue sollempniter est condempnatus petitori ex promissione eidem facta per stipulationem ad restituendum eidem quemdam equum certum ab inde ad.XXX. dies, sub pena.X. librarum communi solvenda» (ASP A.29, ff. 314vb-316ra); si veda Bellomo, Quaestiones in iure civili disputatae, n. 394, p. 199. È utilizzata da Giovanni d'Andrea in Guglielmo Durante, Speculum iudiciale, IV, II. de iureiurando, add. actione, t. 2, p. 182; si veda Sorrenti, Testimonianze di Giovanni D'Andrea, n. 212, p. 119. «Quidam creditor egit contra debitorem» (Collegio di Spagna 81, f. 86r; f. 85r cartulazione antica; inc. Sequitur eiusdem quaestio domini Ni. octava. Quidam creditor egit contra debitorem ad.x.; expl. talis exceptio ut ff. de exceptionibus, l. exceptio- 
te compenetrato all'ambiente dello Studio ${ }^{95}$. Trova conferma, anche tramite questo canale, il forte ruolo di collegamento tra prassi e scuola esercitato da tale genere dottrinario e letterario, attraverso il quale fra XIII e XIV secolo matura un sempre più forte aggancio delle normative municipali alla scienza universitaria, chiamata a individuare un diritto in grado di adeguare la varietà e la mutevolezza dei fatti reali a concetti e rationes generali a fronte dell'assenza di una normativa valida come legge generale e assoluta e per di più lacunosa e parziale nelle sue differenti manifestazioni a livello municipale $^{96}$

Condividendo un percorso battuto da numerosi altri maestri nel corso del pieno Duecento, tra le quaestiones attribuite a Mattarelli ab-

nes, $\S$ finalis (D. 44.1.3) et si messor [rectius: mensor] falsum modum dixerit, 1..i. (D. 11.6.1) secundum dominum Nicolaum); si veda MAFFeI, I codici del Collegio di Spagna, p. 168. «Quaestio de confessione in l. pretor, § docere, ff. vi bonorum raptorum» (Collegio di Spagna 83, f. 148r; inc. Iste paragraphus est positus in 1. pretor, § docere, ff. vi bonorum raptorum (D. 47.8.4.12); expl. que est species diversa a contractu mutui qui re contraitur ergo etc. et hoc disputavit Ny. de Mactarellis quod notat Ia(cobus)); si veda MafFeI, I codici del Collegio di Spagna, p. 222. La quaestio è ripresa in seguito da Baldo: Vat. Lat. 10726, f. 296v; in merito BeLlomo, Quaestiones in iure civili disputatae, n. 68, p. 652. «Quidam petit a Titio centum ex causa mutui ex lite contestati, produxit ystrumentum publicum et sollemne, in quo continetur quod petitor ex causa mutui numeravit» («Quaestio notabilis an duo testes reprobent ystrumentum. Rubrica») (Vat. Lat. 10726, ff. 175r-176r): edizione in Bevilacoua, Una «quaestio» di Nicolò Mattarelli, pp. 151-157, già ricordata in Borino, Codices Vaticani Latini. Codices 10701-10875, p. 125, n. 107. Per il fatto di contenere anche il bartoliano De tyranno, il codice miscellaneo vaticano trascritto nel 1442-43 da Johannes de Guidonibus de Accumulo, assessore del podestà a Corneto, Viterbo, Norcia e Perugia, è ricordato in Quaglioni, Politica e diritto, pp. 87 s. e 239 s., e in ID., Il pubblico dei legisti trecenteschi, p. 198.

${ }_{95}$ Quaestio «Quidam scolaris» riferita da Giovanni d'Andrea in Guglielmo Durante, Speculum iudiciale, IV, III, de locato, add. prima, t. 2, p. 262, e leggibile in ASP A.29, ff. 289vb-290vb. In merito SorrentI, Testimonianze di Giovanni D'Andrea, n. 239, p. 127. Si aggiunga anche la quaestio «Quidam mercator in publico sollempni instrumento fuit confessus se debere cuidam scolari centum ex causa mutui», riportata sempre in ASP A.29, ff. 252ra-253rb, e pure nel ms. Foligno, Biblioteca Jacobilli, A.VI.17, ff. 33v-35r, con varianti nella parte finale (expl. «[...] ad aliam causam trahi non potest, ff. de penis, l. interpretatione (D. 48.19.42). Et ita consulo ego Nicolaus de Mattarelli»). Si veda BEllomo, Quaestiones in iure civili disputatae, n. 338, p. 173 s. e n. 364, p. 188, e anche sopra, cap. II, nota 90.

96 Il tema è largamente approfondito in importanti saggi apparsi negli ultimi decenni, per i quali si veda almeno CORTESE, Il Rinascimento giuridico medievale, $\mathrm{p}$. 66 ss.; Bellomo, Due 'libri magni quaestionum disputatarum'; ID., Aspetti dell'insegnamento giuridico nelle università medievali. 
bondano soprattutto quelle inerenti problemi statutari, legate ai contenuti della normativa municipale e alle numerosissime fattispecie che scaturiscono dalla sua applicazione soprattutto in ambito criminale, ove risulta più urgente chiarire sia numerosi aspetti procedurali, sia modalità e misure nella somministrazione delle pene a vantaggio di un funzionamento efficace delle giustizia pubblica comunale e della sua capacità di affermare un sufficiente livello di legalità ${ }^{97}$.

Anche per tali ragioni, tra i contributi di numerosi giuristi antiqui e moderni citati da Alberico nel proprio opus statutorum, che per ampiezza e sistematicità si può considerare «l'espressione più compiuta della riflessione giuridica medievale sulla legislazione statutaria $\rtimes^{98}$, non mancano quaestiones di Niccolò Mattarelli che

97 I legami tra produzione di quaestiones, soprattutto connesse alla normativa statutaria, la loro frequente traduzione in consilia e il funzionamento delle istituzioni giudiziarie comunali fra Due e Trecento è in particolare esaminato da VALLERANI, Il diritto in questione, pp. 13 ss., 27 ss., ove rileva come in numerosi manoscritti antologici che raccolgono masse di quaestiones vengano copiate anche opere a carattere prevalentemente processuale. Nella produzione di Mattarelli non mancano neppure quaestiones di ambito civilistico ispirate a norme particolari, come la quaestio «Statutum est in civitate quod heres per potestatem mitti debeat in possessionem rerum quas possidebat defunctus tempore mortis»: in ASP A.29, ff. 278va-279rb e in Chigi E.VIII.245, f. 140ra-va (qui attribuita a Nicola da Cremona); edizione parziale in Bellomo, Giuristi cremonesi e scuole padovane, p. 152 ss.; si veda ID., Factum proponitur certum, p. 3, nota 7, e ID., Quaestiones in iure civili disputatae, n. 354, p. 183 e n. 202, p. 301 s. In merito anche la quaestio «Statutum est in civitate quod filia familias decedente matrimonio liberis extantibus dos a patre profecta perveniat ad liberos mulieris»: ASP A.29, ff. 288rb-289vb e Chigi E.VIII.245, f. 129ra-vb (si veda Bellomo, Quaestiones in iure civili disputatae, n. 363, p. 187 e n. 182, p. 292 s.).

98 Quaglioni, Legislazione statutaria e dottrina della legislazione, p. 42. Si rinvia a Bellomo, Aspetti dell'insegnamento giuridico, p. 51 ss. e ID., I fatti e il diritto, pp. 529 ss., 545 ss. e 554 ss. per la qualificazione tramite l'appellativo di moderni dei doctores iuris attivi dai primi anni Settanta del Duecento contrapposti agli antiqui riferibili piuttosto agli anni centrali dello stesso secolo. Oltre che puramente temporale, la distinzione si basa anche sulla strumentazione logica che differenzia i doctores antiqui da quanti vengono considerati moderni oppure moderniores ed è contraddistinta dall'analisi delle potenzialità conoscitive non delle singole cose, ma di più complesse proposizioni verbali esaminate tramite la loro espressione in forma di consequentiae secondo tecniche e schemi argomentativi che si distaccano dall'aristotelismo tradizionale: ERrERA, Il concetto di scientia iuris, pp. 96 ss., 135 ss.; MARTino, Argumenta ex legibus, in part. pp. 149, 154 ss.; Padovani, A proposito di, p. 434 ss.; ID., Modernità degli antichi, p. 150 ss. In forma assai sbrigativa, un giurista al passo con i tempi come Cino taccia senza riserve la Glossa di incompletezza e scarsa attendibilità: Doctores moderni di- 
approfondiscono tipologie diverse di violenza privata ${ }^{99} \mathrm{o}$ che vengono comunque ricordate da altri giuristi - in primo luogo Alberico da Rosciate - nel contesto della catena di opinioni dottrinarie cui si appoggia la linea argomentativa da essi seguita ${ }^{100}$.

cunt, quod glossa minus plene et minus vere loquitur; e i moderni sono identificati direttamente con i maestri ultramontani: Moderni ut Iac. de Ra. \& Pet. non approbant [...] (CINO DA PISTOIA, Lectura super Codice, in lib. VII, tit. XLVII De sententiis, f. 459ra e 461rb).

99 Quaestio «Duo fratres videntes duos suos inimicos transire ante domum eorum insurrexerunt in eos et, eis in terram deiectis, cum manibus et pedibus fortiter percusserunt. Isti percussi quilibet per se accusant percussores, 1. Iul. de vi privata, vel ex forma statuti loquentis de percussionibus. Quaeritur an percutientes bis condemnari debeant an semel tantum» (Alberico da Rosciate, Commentarium de Statutis, 1. II, q. 32, f. 33vb-34rb). Alberico precisa che si tratta della quinta questio di una serie che inizia a f. 33rb dedicata a varie fattispecie penali con profili comuni: reati diversi ma in qualche forma tra loro connessi oppure la reiterazione di un medesimo reato e le conseguenti sanzioni previste dalle norme statutarie. Oltre che in ID., Commentaria, de modo multarum, 1. eos, qui ordinario (C. 1.54.6), f. 68va, n. 8, la medesima quaestio è ripresa da Giovanni d'Andrea in Durante, Speculum iudiciale, IV, IV, de penis, add. prelibatum, t. 2, p. 516, e richiamata anche ibidem, IV, I, de libellorum conceptione, add. defensionibus, t. 2, p. 76; si veda SORRENTI, Testimonianze di Giovanni D'Andrea, n. 357, p. 165, richiamata pure a n. 186, p. 110; Romano, Le quaestiones disputatae, n. 48, p. 101, richiamata pure a n. 86, p. 123. La quaestio è trascritta in ASP A.29, ff. 267ra-268vb, su cui si veda Bellomo, Quaestiones in iure civili disputatae, n. 346, p. 178. Quaestio publice disputata «In statuto dicitur quod qui vulneraverit cum lancea, condemnetur in centum, qui aliter in quinquaginta, et pro vulneribus factis de nocte penae duplicentur. Duo accusati sunt, quod vulneraverant Titium cum lanceis, testes producti dicunt quod viderunt tali nocte accusatos et quod unus eorum ipsum vulneravit. Sed quis eorum fuerit, et utrum cum cultello vel lancea, discernere non potuerunt propter noctem. Quaeritur an accusati debeant condemnari et si debeant condemnari, in quantum» (ALBerico da Rosciate, Commentarium de Statutis, 1. II, q. 44, f. 35rb-vb; richiamata anche in ID., Commentaria, de infamibus [de his qui notantur infamia], 1. quid ergo, § pena gravior (D. 3.2.13.7), f. 194ra, n. 30. Un esemplare manoscritto si trova in ASP A.29, ff. 268vb-270ra, per cui si veda Bellomo, Quaestiones in iure civili disputatae, n. 347, p. 179). È ricordata in RomANo, Le quaestiones disputatae, n. 49 , p. 101.

100 Vengono ricordate la due quaestiones di Mattarelli «Quidam occisus»e «Statuto cavetur» in chiusura della quaestio affrontata da Alberico «Quinque erant in una domo, unus eorum reperitur interfectus, quaeritur, cum nesciatur per quem, an omnes debeant puniri pena statuti vel nullus» (Alberico da Rosciate, Commentarium de Statutis, 1. III, q. 61, f. 64ra-b). La seconda tra le due quaestiones era stata già in precedenza utilizzata da Alberico (ibidem, l. II, q. 44, f. 35rb-vb; cfr. sopra, nota 99) e ricordata pure nel Commentario al Digesto vecchio con il titolo «In statuto cavetur quod qui alium vulneraverit»: ID., Commentaria, de infamibus [de his qui notantur infamia], 1. quid ergo, § pena gravior (D.3.2.13.7), f. 194ra, 
Da questo quadro abbastanza frammentato di testimonianze, se non può certamente filtrare una visione d'insieme del pensiero di Mattarelli in campo penale, emerge tuttavia una tendenza prudente nel delimitare la portata delle norme stabilite ex forma statuti come nel caso delle percosse inferte da più soggetti ad altrettante vittime, risolta inquadrando la fattispecie come crimine pubblico, cui deve conseguire una sola imputazione in capo a ciascuno dei responsabili e, come ulteriore effetto, una sola sanzione ${ }^{101}$.

In ossequio a un principio generale per il quale in criminali vero agitur pro bono publico, ut maleficia puniantur, si ammette che due soggetti accusati di avere procurato di notte ferite a un terzo armati di lancia vengano effettivamente sottoposti a condanna. Nonostante l'esame di tre condizioni a discarico degli imputati prospettate da Alberico (la difficoltà di stabilire chi abbia realmente inferto $i$ colpi, l'incertezza nel determinare se le ferite siano state procurate con lance o con coltelli, la mancata indicazione nel libello di accusa dell'ora precisa in cui si sarebbe svolto il fatto), la solutio prospettata da Mattarelli richiede in via generale che i responsabili condemnari debeant de vulnere facto cum lancea per iura ad hoc adducta $\mathrm{e}$ per di più quod pena debeat duplicari propter noctem per iura pro hac parte adducta ${ }^{102}$. È uno dei casi in cui la norma statutaria po-

n. 30. Su quest'ultima si veda Bellomo, Quaestiones in iure disputatae, n. 347, p. 179. Le due quaestiones sono censite in Romano, Le quaestiones disputatae, n. 49, p. 101 e n. 125, p. 147.

101 Alberico da Rosciate, Commentarium de Statutis, 1. II, q. 32, f. 33vb34rb: a f. 34rb la solutio, conclusa con il richiamo al nome del maestro, Nicolaus. $\mathrm{Si}$ vedano anche le due quaestiones di ambito criminale «Quidam accusabatur de aliquo crimine ex quo per legem debebat imponi pena corporalis, set per legem municipalem imponitur peccuniaria» e «Quidam accusabatur de quodam delicto et pendente accusatione mortuus est accusator» (vedi sotto, nota 129), risolte entrambe da Mattarelli circoscrivendo la portata della lex municipalis, nell'un caso ammettendo l'applicazione della pena corporale sancita dalla lex anziché di quella pecuniaria prevista dal diritto proprio e nell'altro rimarcando l'annullamento della condanna penale in seguito alla sopravvenuta morte dell'accusatore, all'insaputa del giudice, che invalida tutti gli atti successivi sulla base della l. si quis, C. si reus vel accusator mortuus fuerit (C. 9.6.6) e della l. libellorum, ff. de accusationibus, $\S s i$ (D. 48.2.3.4).

102 Ibidem, 1. II, q. 44, f. 35rb-vb. Il tema del raddoppio della sanzione penale in caso di crimine compiuto nelle ore notturne, così come sancito di frequente dalla normativa statutaria, e la conseguente riflessione dottrinaria concentrata sull'indi- 
sta alla radice della quaestio viene sottoposta all'interpretazione di principi più generali anche in consonanza con l'opinione di una più larga corrente dottrinaria di multi tamen et magni doctores - tra $\mathrm{i}$ quali vengono espressamente ricordati Dino e lo stesso Niccolò concordi nel ritenere che il presunto reo debba essere assolto se tuttavia l'accusatore non prova con esattezza la qualità delle ferite subite al fine di stabilire con quale arma siano state inferte e se vi sia stata o meno effusione di sangue ${ }^{103}$.

La considerazione di istituti tipici del diritto proprio emerge con evidenza in relazione al tema del bando, cui Alberico - come ben noto - riserva l'intero quarto libro del proprio opus statutorum aprendolo con una questio di carattere generale, incentrata sulla definizione stessa dell'istituto e sulla sua origine, che già era stata di Niccolò Mattarelli ${ }^{104}$. Proprio lui a Padova, assieme a Iacopo Belvisi, aveva rivolto specifica attenzione alla problematica scottante del bando e dei suoi effetti, considerata la larga applicazione dell'istituto nella dinamica della politica comunale, senza tuttavia manifestare interesse nei confronti del suo uso come strumento di lotta politica ${ }^{105}$.

Sulla problematica generale ripresa da Alberico il pensiero del docente modenese si dipana seguendo i capisaldi di un filone consolidato a livello dottrinario che riconosce all'istituto un duplice profilo: a tenore del diritto comune esso è l'effetto di una sentenza giudiziaria contro il contumace usque ad relegationem, mentre secondo

viduazione precisa delle due fasi di luce e di buio nell'arco temporale della giornata sono in particolare approfonditi da MonTANOS FERRIN, «An de die vel de nocte».

103 Una quaestio ritenuta esemplare da Storti Storchi, Prassi, dottrina ed esperienza legislativa, p. 447 e p. 451 per l'interpretazione dello statuto mediante principi del diritto comune convalidati dal consenso unanime della dottrina. Si veda anche la quaestio «Quidam accusabatur de aliquo crimine ex quo per legem debebat imponi pena corporalis, sed per legem municipalis imponitur peccuniaria», che Mattarelli, sviluppandola probabilmente a margine di una sua lectura, risolve in senso sfavorevole all'applicazione della sanzione prevista dalla lex municipalis: ASP A.29, f. 314va-b; si veda Bellomo, I fatti e il diritto, p. 381; ID., Quaestiones in iure civili disputatae, n. 392, p. 199.

104 Alberico da Rosciate, Commentarium de Statutis, 1. IV, q. I, ff. 65vb66rb. La materia trattata nell'ultima parte dell'opera di Alberico, articolata in 114 questioni, è complessivamente analizzata in Storti Storchi, Prassi, dottrina ed esperienza legislativa, p. 469 ss.

105 Esempi delle quaestiones dedicate da Iacopo Belvisi e da Mattarelli al tema specifico sono analizzate da Bellomo, I fatti e il diritto, p. 661 ss. 
consuetudine e diritto municipale fere totius Italiae esso può anche oltrepassare i limiti di tale sanzione ed essere comminato in seguito a gravi crimini di qualsiasi entità ${ }^{106}$. Di esso Mattarelli fornisce anche una curiosa paretimologia derivando la parola da banderia id est parvo vexillo, ovvero una pubblica insegna militare utilizzata in battaglia dall'autorità imperiale, ciò che ne legittima il significato di pubblica espulsione ab honoribus et officia et statu civitatis comminata sia contro soggetti in quanto contumaci sia di frequente propter delicta, cui consegue la deiectio e l'interdictio dalla comunità cittadina dichiarate in forme pubbliche tramite banditori o pubblico editto e perciò definite come bando ${ }^{107}$. Analoga origine del termine viene ricordata da Alberico in altra sede precisando che si tratta di un vocabolo largum et obscurum, il cui significato effettivo in massima parte è determinato per statuta vel consuetudinem civitatum ${ }^{108}$.

106 È frutto della speculazione dei Glossatori avere esaminato l'istituto dell'esilio romano nelle forme soprattutto della relegazione e della deportazione, mentre è soltanto circa dalla metà del Duecento che matura l'attenzione al bando come istituto di diritto proprio utilizzando in prevalenza gli schemi della relegazione, che permette ai condannati di rimanere titolari di beni e diritti propri, come la capacità di testare, di succedere in testamento e di agire in giudizio: CAVALCA, Il bando nella prassi e nella dottrina, p. 78 ss. Nella dinamica sociale e istituzionale del Comune di Siena l'applicazione di tale istituto è stata studiata, grazie allo spoglio dei registri giudiziari duecenteschi, da PAZZAglins, The criminal ban of the Sienese Commune, in part. p. 26 ss. per la procedura seguita dalle autorità comunali nella comminazione del bando e la sua evoluzione tra Due e Trecento. In anni recenti la più complessiva analisi del bando come strumento punitivo del reato politico, nei casi del Comune di Bologna e di altre città dell'Italia centro-settentrionale, si deve a MiLANI, Prime note sulla disciplina del bando a Bologna; ID., L'esclusione dal Comune; ID., Banditi, malesardi e ribelli.

107 Di tale derivazione del lemma in questione non si riscontrano tracce nei più collaudati lessici del tempo riconducibili a Papias, Uguccione da Pisa e Giovanni Balbi, dai quali sono completamente assenti i lemmi 'Bando' e 'Bandiera'. Si vedano Papias Vocabulista, Elementarium; Balbi, Catholicon; Riessner, Die Magnae Derivationes des Uguccione da Pisa, opera composta dal celebre canonista intorno al 1190, a conclusione del suo insegnamento bolognese e prima di assumere la guida della diocesi di Ferrara: Mercati, Sul luogo e sulla data di composizione delle «Derivationes», p. 493. Neppure Bartolo si dimostra interessato ad approfondire la funzione delle bandiere come insegne nel suo noto trattato sul linguaggio araldico: Cavallar, Degenning, Kirschner, A grammar of signs, pp. 109-121 per l'edizione del testo.

108 Alberico da Rosciate, Dictionarium iuris, f. 32rb, v. Bannum. È noto che questa opera di Alberico, già terminata alla metà del Trecento, incorpora un nucleo derivato dall'analogo dizionario predisposto da Jacques de Révigny: D’AmELIO, 
Oltre a ricordare il parallelo, già istituito da Dino e che sarà poi tranquillamente confermato da Baldo, tra bando e scomunica, in quanto sanzione che conduce all'espulsione dalla comunione con la Chiesa e, di conseguenza, dalla comunità dei fedeli ${ }^{109}$, nella quaestio ripresa da Mattarelli si sottolinea che sono gli statuti comunali a dare forma a questo istituto particolare, che prevede la relegazione e l'espulsione dal distretto di competenza del giudice che lo emana e la deposizione da ogni pubblico ufficio ${ }^{110}$.

La pena del bando si può comminare pro delicto aut pro contumacia. Nel primo caso essa, pronunciata sia in presenza che in assenza del reo, ha il duplice effetto della privazione degli incarichi pubblici e dell'espulsione dalla civitas e dal distretto del giudice, e può essere anche integrata dalla confisca dei beni esplicitamente dichiarata, che non era invece prevista nella relegazione dal diritto romano. Nel secondo caso, se non viene stabilita la confisca dei beni il bando ha unicamente l'effetto cuiusdam publice excommunicationis secularis che si può revocare comparendo in giudizio; se invece

Il Dictionarium iuris di Jacques de Revigny. In anni recenti è stata anche messa in discussione l'autenticità del testo del giurista bergamasco che la tradizione ha tramandato come tale, confluito nell'editio princeps bolognese del 1481 rapidamente seguita dalla stampa milanese del 1482 e da quella pavese del 1498: per la discussione si veda CONETTI, La dottrina dell'impero, p. 321.

109 Ghisalberti, La condanna al bando, p. 37 ss.; Cavalca, Il bando nella prassi e nella dottrina, p. 88, e cfr. Alberico da Rosciate, Commentarium de Statutis, 1. IV, q. 1, f. 66ra, n. 8. Per la ripresa da parte di Baldo si veda BALDI UbaLDI, In sextum codicis librum commentaria, f. 69va (ad C. 6.24.1), ove si richiama esplicitamente l'opinione di Mattarelli sul fatto che «bannitio eiicit hominem extra bonum publicum, ut gaudere non debeat auxilio reipublice nec officis publicis, nec iurisdictione iudicis adiuuvetur, secundum Nico. de Ma».

110 Già nei primi anni del secolo XIII Guizzardino da Bologna sottolinea la forza dello statuto locale quale fonte normativa per giustificare la condanna al bando del presunto reo contumace: «Sed quid si ille contumax, etiam multa indicta, non veniat, nec in ea prestanda pareat? Respondeo quod ponat eum in banno suae civitatis secundum loci consuetudinem quae pro lege habetur» (PAlmieri, Pillii libellus de preparatoriis litium, p. 24; l'attribuzione a Guizzardino anziché a Pillio da Medicina si deve a Enrico Besta nell'ambito di una più complessiva rivalutazione dell'opera del giovane giurista bolognese: in merito BESTA, Su due opere sconosciute, p. 7 ss.; ID., Per la storia della nostra letteratura processuale. Ancora un secolo più tardi Alberto Gandino fonda nella consuetudo Lombardorum la legittimità della condanna al bando perpetuo per il presunto reo contumace: GANDINo, Questiones statutorum, q. LVIII Bannum perpetuum an possiti dari alicui secundum consuetudinem Lombardorum, p. 183. 
viene comminata pure la confisca, esso conserva un effetto duplice e quest'ultima non è revocabile pur cessando la contumacia. Queste forme, tuttavia, non esauriscono tutti gli effetti del bando che si determinano nella prassi, poiché molte altre si possono avere secundum diversa statuta et consuetudines civitatum.

Su alcuni corollari più specifici dell'istituto del bando Mattarelli ritorna nella formulazione di altre quaestiones che toccano punti nodali circa le condizioni di quanti incorrevano in tale sanzione pro maleficiis, quali i diritti ereditari e l'eventuale impunità per chi li avesse uccisi. Considerate le differenze tra bando e deportazione, i banditi possono essere nominati eredi anche dopo la confisca dei beni e il patrimonio così acquisito può passare ai rispettivi successori, inclusi beni già detenuti in alia civitate, ovvero entro un distretto diverso da quello ove è stato emanato il bando ${ }^{111}$.

Sul problema della confisca dei beni detenuti dal bandito in distretto alieno il giurista modenese si era già pronunciato in un'altra quaestio publice disputata risolvendola in senso negativo ${ }^{112}$. Sul tema invece dell'impunibilità per quanti avessero penalmente offeso un bandito, anche nel caso si trattasse di un chierico, l'opinione di Mattarelli sembra concordare con quella di Iacopo d'Arena, Dino et multi alii doctores nel cui solco si colloca pure Alberico da Rosciate,

111 Alberico da Rosciate, Commentarium de Statutis, 1. IV, q. 13, f. 67vb: «Et quia ista sunt utilia et continua et habita est mentio de questione domini Nic. de Mata. qui multum declarat praesentem materiam [...] Et quaestio talis est: Quidam bannitus fuit de homicidio et eius bona omnia publicata, post quem ipse ab aliquo haeres institutus, adita haereditate decessit. Nunc haeres eius contendit de bonis, que ipse bannitus in alia civitate habebat, et de aliis bonis praedictae haereditatis ei delatae. Quaeritur an ipsa bona ad ipsum haeredem debeant pertinere an ad alium et si ad alium ad quem».

112 Ibidem, 1. III, q. 2, f. 55ra-va: «Quaero, quia dictum est forensem debere puniri pena statuti, an si ex forma statuti ex delicto debeant bona publicari an publicari poterunt bona quae habet in alieno districtu», ove Alberico recupera la quaestio publice disputata da Mattarelli «Quidam inculpatus de homicidio» (f. 55va, in fine), riportata in due codici vaticani: ASP A.29, ff. 273vb-276ra e Chigi E.VIII.245, ff. 108vb-109rb (ma con diverso explicit); si veda Bellomo, I fatti e il diritto, pp. 142, 146, nota 224, 302; ID., Quaestiones in iure civili publice disputatae, n. 351 p. 181 e n. 146, p. 276. La quaestio è utilizzata da Giovanni d'Andrea in Guglielmo Durante, Speculum iudiciale, II, I, de cessione actionis, add. l. fin., t. 1, p. 385 e ibidem, III, I, de accusatione, add. ut intra certum tempus, t. 2, p. 20. Si veda Sorrenti, Testimonianze di Giovanni D'Andrea, n. 173, p. 104 e n. 61, p. 63. 
il quale sul punto riprende una quaestio de facto già esaminata da Alberto da Gandino durante la sua attività di assessore del podestà a Siena ${ }^{113}$ partendo dalla constatazione formale che devono essere considerati banditi soltanto coloro i cui nomi sono inseriti negli appositi registri comunali (in libris clavium dicte civitatis) ${ }^{114}$.

La riflessione sviluppata da Mattarelli in via diretta sul tema punta a privilegiare l'impiego di categorie generali quali criteri sicuri in grado di sostenere l'interpretazione dei fatti concreti desunti dall'esperienza materiale. Ponendosi il dubbio sulla validità dello statuto nel legittimare la non punibilità dell'omicida del bannitus, egli preferisce piuttosto scindere la quaestio iuris nei singoli atti che ne costituiscono la fattispecie e ribaltare il nucleo del problema interrogandosi sulla possibilità che l'omicida possa restare sine pena ratione statuti. La ratio dello statuto può essere tale da legittimare l'impunità per chi si macchi di un omicidio ma può anche essere messa in discussione, al pari di qualsiasi contingenza fattuale, per dubitare della sua validità a fronte invece delle categorie assolutamente stabili garantite dal ius commune $e^{115}$.

Per argomentare sul problema Mattarelli pone al centro della riflessione la serie di condizioni attraverso le quali si può meglio definire, nei suoi molteplici profili, la fattispecie considerata: in primi luogo, se si può effettivamente provare trattarsi di omicidio e se, in

113 Quindi collocabile durante il primo semestre del 1299, dopo che il Gandino era entrato ufficialmente in carica l'8 dicembre dell'anno precedente: QuAGLIONI, Gandino Alberto, p. 943.

114 Alberico da Rosciate, Commentarium de Statutis, 1. IV, q. 29, ff. 70va71 ra. Come ricorda Baldo, già Martino Sillimani precisava che il bando dovesse essere esemplato in libris comunis per tabellionem, e se ciò non fosse avvenuto per errore del notaio, a parere di Cino, ne sarebbe stato viziato il bando stesso; mentre il notaio, secondo Iacopo Belvisi, avrebbe potuto sanare il proprio errore non tramite una correzione apportata sua authoritate, ma unicamente mandato superioris: BALDI UbALDI, In sextum codicis librum commentaria, f. 66rb, n. 3-4 (ad C. 6.23.24).

115 La quaestio «Statutum est in civitate quod banniti possint impune occidi» è leggibile in ASP A.29, ff. 265vb-267ra; si veda Bellomo, Quaestiones in iure civili disputatae, n. 345, p. 177 s. La quaestio, che affronta un tema generale e ricorrente in merito all'esenzione dalla pena per quanti infliggono un danno, mediante ferite, o direttamente la morte a chi è stato bandito, è analizzata in particolare in BELLoмо, 'Factum' e 'ius', p. 88 s.; ID., Factum proponitur certum, p. 27; ID., I fatti e il diritto, p. 664 s. La riflessione della dottrina bassomedievale sul punto specifico, a partire dai Glossatori, è ricostruita da GHISALBERTI, La condanna al bando, p. 69 ss. 
base a due testimonianze concordi, si può provare la notorietà del reato contestato al presunto responsabile. Da cui consegue anche la riflessione sul valore della testimonianza, che è tale perché in grado di riportare fatti osservati direttamente e non riferiti in base alla sola notizia pubblicamente circolante: testes debent testificare de sua sciencia quam habent presenciam suam, quia fuerunt presentes facto, non de alieno ${ }^{116}$.

Scandita tramite una minuta analisi degli elementi che possono intervenire a definire la situazione di fatto e arricchita dal fitto richiamo a passi delle leggi romane, con ricorrente citazione della lex Aquilia e della responsabilità penale da essa riformulata all'interno dell'ordinamento romano ancora in età repubblicana ${ }^{117}$, l'analisi di Mattarelli si muove unicamente sul piano dei fattori concreti, soggettivi e oggettivi, che possono rilevare in merito alla quaestio iniziale convergendo nel riconoscere sostanziale validità al dettato statutario. A parte il caso in cui l'omicidio del bandito sia compiuto dolosamente, ciò che deve essere punito, il responsabile debet absolvi tanto nel caso sia consapevole dello stato ecclesiastico della vittima quanto ne sia all'oscuro, dal momento che la condizione di quest'ultima non appare più rilevante dopo che ne sia stato riportato il nome in libris bannitorum, cosa che equivale alla sostanziale rinuncia all'appartenenza all'ordo clericalis ${ }^{118}$.

Le riflessioni maturate in precedenza da Iacopo d'Arena sull'analogo tema del bando e dei suoi effetti avevano preso forma, negli ultimi decenni del Duecento, sia in una quaestio disputata riferibile

\footnotetext{
116 ASP A.29, f. 266va.

$117 \mathrm{Si}$ contano otto allegazioni di tale complesso normativo, con riferimento ai brani dottrinari notoriamente riuniti sotto il titolo II del IX libro del Digesto. Sulla specifica legislazione, in una prospettiva concentrata unicamente sull'esperienza e sulle fonti di età romana, si rinvia agli studi recenti di Zıьıтто, L’imputazione dal danno aquiliano; Corbino, Il danno qualificato e la lex Aquilia, in specie p. 41 ss.; Schipani, Contributi romanistici, pp. 41 ss., 63 ss., 133 ss.; Rossetti, 'Poena' e 'rei persecutio'; DesAnti, La legge Aquilia. Osservazioni in merito alle riflessioni della dottrina di età medievale e moderna intorno ad alcuni aspetti del danno aquiliano si devono a CuRsi, Iniuria cum damno, in part. p. 29 ss. Nello svolgimento della quaestio da parte di Mattarelli lo strettissimo dialogo con le fonti romanistiche è accentuato dalla totale indipendenza rispetto a differenti opzioni dottrinarie, mancando qualsiasi allegazione del pensiero di altri giuristi.

118 ASP A.29, ff. 266vb-267ra.
} 
al problema dell'impunità da riconoscersi a quanti uccidono un bandito, ben nota e citata da Alberico ${ }^{119}$, sia nella redazione di un trattatello sulla materia che costituisce la prima riflessione organica volta a esaminare i molteplici profili di tale istituto. In questa operetta il giurista, allievo di Guido da Suzzara e docente per molti anni a Padova ${ }^{120}$, insiste sulla distinzione tra consuetudine e leggi municipali, generalmente inclini ad ammettere l'omicidio impunito dei banditi, e ius commune, che invece, recuperando la similitudine con la relegazione e la deportazione romana, non ammette che essi vengano offesi impunemente, a parte alcuni casi particolari ${ }^{121}$. Su questa linea prudente si colloca la riflessione di Iacopo, diversamente dalla contraria opinione ribadita dal Gandino richiamando la catena di pareri favorevoli alla liceità delle offese nei confronti dei banditi ${ }^{122}$. Ed egli vi aderisce sottolineando tuttavia il

119 Il quale la ricorda con precisione in chiusura della propria quaestio ricordata sopra, a nota 260 (f. 71 ra) e anche in Alberico da Rosciate, Commentaria, in ius vocati, l. item pro patrona (D. 2.6.2), f. $115 \mathrm{rb}, \mathrm{n}$. 23. Altre quaestiones di Iacopo d'Arena riferite da Alberico in ID., Commentarium de Statutis, l. IV, q. 4, f. 66vb, n. 7. Si aggiunga un'altra quaestio dedicata al problema della legittimazione del bandito ad agire, una volta cancellato il bando, per la riparazione delle offese subite, di cui dà notizia Giovanni D’Andrea in Guglielmo Durante, Speculum iudiciale, III, I, de accusatione, add. ut intra certum tempus, t. 2, p. 18; la quaestio è ricordata in CAVALCA, Il bando nella prassi e nella dottrina, p. $92 \mathrm{~s}$.

120 Quaglioni, Dell'Arena (D'Arena) Jacopo, p. 243 s.; ID., Iacopo d'Arena, p. 1100.

121 Iacopo D’Arena, Tractatus de bannitis, f. 355vb-356ra, n. 16, ove per tali eccezioni richiama C. Quando liceat sine iudice unicuique vindicare (C. 3.27.1 e 2) e C. De desertoribus (C. 12.45), precisando altresì che quanto vi è disposto non deve applicarsi ai banditi «arg. 1. cum quidam de libe. et posth. (= D. 28.2.19) imo favorabiliter est interpretandum, ut l. interpretatio(ne) de pen. (D. 48.19.42) \& 1 . quotiens (D. 48.19.1 pr.) de reb. dub. et talis interpretatio esset ambitiosa, quia homines vita privaret et aliis materiam delinquendi daret. Immo, quod plus est, talia statuta hoc expresse permitentia non valent de iuris subtilitate». Vari passaggi del trattato di Iacopo d'Arena, l'unico sul tema del bando a confluire nel TUI assieme a quello tardo quattrocentesco di Nello da S. Gemignano (TUI, XI/1, ff. 357r-406v: sull'autore e la sua opera si veda in particolare BASSANI, Note a margine), sono analizzati da GHisAlberti, La condanna al bando, p. 16 s. e passim.

122 Ibidem, p. 71 ss. Un atteggiamento improntato a una notevole rigidità nella repressione penale che traspare anche dalla diretta esperienza del Gandino come giudice del podestà a Bologna nel 1289 e poi del Capitano del Popolo nel 1294; atteggiamento che viene mitigato in vari casi all'interno dei processi da lui istruiti, come pure per molte altre cause in materia di bando, dai consilia formulati da autorevoli giuristi bolognesi maggiormente inclini a valorizzare le tutele a favore dei rei e a creare «una rete di assistenza di altissima qualità per i cives bolognesi»: VAL- 
fatto che la condanna e i suoi effetti, e quindi la possibilità di subire offese senza poterne sanzionare i responsabili, andrebbe commisurata alla condizione posseduta dai banditi anteriormente all'emanazione della sentenza anziché a quella, ben diversa, conseguente al bando, secondo una interpretatio ambitiosa e comunque favorevole al condannato in una materia che il giurista ammette assai delicata, quia homines vita privaret et aliis materiam delinquendi daret ${ }^{123}$.

\section{Libri magni quaestionum}

La rilevanza di Niccolò Mattarelli come autore di quaestiones, sebbene all'interno di una schiera di giuristi largamente rappresentata fra Due e Trecento, emerge con forte spessore attraverso la testimonianza di due ben noti manoscritti vaticani risalenti alla prima metà del secolo XIV e oggetto di pluridecennali ricerche da parte di Manlio Bellomo.

Il primo di essi (ASP A.29) risulta formato da masse omogenee di testi riferibili, talora anche in modo apertamente dichiarato, a un solo giurista oppure attribuiti ad autori diversi ma riuniti assieme sulla base di altri criteri. Una prima e consistente parte del codice dimostra la sedimentazione di quaestiones formatesi soprattutto in ambiente bolognese e dovute a numerosi giuristi in un arco di tempo considerevole, tra gli ultimi tre decenni del Duecento e il quartoquinto decennio del Trecento e imperniate, per quest'ultimo periodo, sulla scuola del celebre Iacopo Bottrigari ${ }^{124}$.

LERANI, Consilia iudicialia, pp. 139 s., 142 s. L'orientamento fortemente punitivo del Gandino si riflette nell'architettura procedurale da lui costruita nel Tractatus de maleficiis, ove egli sfrutta un'ampia conoscenza sia dei più noti doctores civilisti del secolo XIII sia della normativa canonistica, in cui affondano le radici del metodo inquisitorio, per giustificare una nuova logica giuridica «che tende a privilegiare la pena come mezzo di difesa della respublica»: si veda l'approfondita analisi di VALLERANI, Il giudice e le sue fonti, a p. 41 per la citazione.

123 Una linea prudente seguita da Iacopo d'Arena che sembra emergere, in base al solo incipit, anche dalla quaestio disputata «Statuto vel consuetudine civitatis habetur quod non fiat ratio de occisione banniti», che gli attribuisce AlberICO DA Rosciate in Commentaria, de adquirendo rerum dominio, 1. in laqueum (D. 41.1.55), f. 75va, n. 3.

124 Bellomo, I fatti e il diritto, p. 156 ss. per il dettagliato esame dei blocchi testuali che compongono le due masse e risultano variamente alternati nello svi- 
Ad essa fa seguito una serie di quaestiones di giuristi padovani che prende avvio a f. 252ra con un testo di Mattarelli, il cui nome viene specificato nella subscriptio a f. 252rb. Da questo esemplare inizia una prima e breve sequenza a base antologica sino a f. 264ra, che include pure un'unica quaestio riferita al figlio Francesco ${ }^{125}$, cui segue sino a f. $290 \mathrm{vb}$ una serie di quaestiones tratte invece da una raccolta organizzata in forma regolare che si apre solennemente, evidenziata da un'apposita rubrica, con un nucleo di 21 quaestiones publice disputatae in studio paduano per dominum Nicholaum de Materello. Ciò che attesta come il redattore del codice seguisse un modello formato da una compatta raccolta di quaestiones del giurista lungamente attivo nello Studio veneto che «doveva essere una raccolta piuttosto rifinita, diffusa e importante» ${ }^{126}$. In seguito, dopo un ampio spazio lasciato in bianco tra i fogli $290 \mathrm{v}$ e $291 \mathrm{v}$, ricompaiono testi redatti ancora da autori padovani che rappresentano una delle ultime masse antologiche del codice vaticano e prendono avvio con una repetitio - o frammento di lectura - sempre di Mattarelli sul tema dell'appello nota pure grazie ad altri due codici iberici ${ }^{127}$.

luppo del manoscritto, la cui descrizione codicologica si deve a Livia Martinoli in Bellomo, Quaestiones in iure civili disputatae, Appendice D, pp. 757-762. La serie compatta di 39 distinctiones di Giovanni Calderini contenute nei primi fascicoli, uniti al restante materiale nel secolo XV, è studiata in particolare da BeLlomo, Saggio sulle distinctiones di Giovanni Calderini. Sul codice e i suoi contenuti, confrontati con quelli analoghi del ms. Chigi E.VIII.245, si veda anche ID., Due 'libri magni quaestionum disputatarum', p. 249 ss.; VALLERANI, Il diritto in questione, p. 18 s.; WeIJERs, Queritur utrum, p. 153 s. Tra i molti giuristi è presente anche Martino Sillimani con 48 quaestiones, 16 delle quali sono riprodotte nel ms. Chigi E.VIII.245, 18 sono riprese da Giovanni d'Andrea, allievo di Martino a Bologna e verosimilmente in possesso di una raccolta di quaestiones del proprio maestro, e 13 da Alberico da Rosciate: Migliorino, «Dominus meus in legibus».

125 ASP A.29, ff. 261ra-262rb. Vedi sopra, nota 13.

126 Bellomo, I fatti e il diritto, p. 118 s., da cui la citazione, e p. 172 ss. Le quaestiones di Mattarelli riportate nel codice ASP A.29 sono analiticamente citate nelle note precedenti, con l'aggiunta di altre due quaestiones: «Quidam nobilis contendit in iudicio imperium, iurisdictionem et omnem potestatem ad se pertinere in quodam castro» (vedi sopra, nota 87 ); «Quidam possidebat quendam fundum quem per plures annos possederat tamquam suum et cum quidam ecclesia contendet dictum fundum esset suum [...]» (ASP A.29, ff. 285va-287ra = Chigi, E.VIII.245, ff. 75va-76rb; si veda Bellomo, Quaestiones in iure civili disputatae, nn. 360-361, p. 186 e n. 91, p. 254).

127 ASP A.29, ff. 292ra-294ra: repetitio o frammento di lectura ad C. de temporibus appellationum (C. 7.63): Bellomo, I fatti e il diritto, p. 174; ID., Quaestio- 
In merito alla compatta serie di quaestiones attribuite al giurista modenese e trascritte nel codice vaticano, l'attenta analisi delle forme testuali porta a identificarle non soltanto come publice disputatae, ma anche come testi scaturiti dalla lettura del Maestro e fissati grazie all'opera di un reportator attento a cogliere con perspicacia le espressioni da lui formulate in prima persona, presumibilmente al momento stesso di conservarne diretta memoria scritta ${ }^{128}$.

Le quaestiones, o forse soltanto una parte di esse, potrebbero quindi essere state tratte «dai margini di un qualche esemplare dei libri legales, dove stavano accanto le une alle altre glossae redactae e glossae reportatae», cosa che comunque porta a concentrare l'attenzione sul mondo dello Studio, soprattutto in ambito padovano, e sulla circolazione di codici legati in modo diretto all'attività didattica di Mattarelli. Al quale si possono riferire, nella parte finale dello stesso codice, altre quaestiones reportatae che non trovano origine in pubbliche dispute, ma scaturiscono piuttosto dall'esegesi condotta dal maestro nell'ambito di una lectura riprendendo anche quaestiones sviluppate da altri giuristi e, nel caso, riferite succintamente dal reportator omettendo le serie dei contrapposti argomenti ${ }^{129}$.

nes in iure civili disputatae, n. 365, p. 188. Essa è riportata in altri due manoscritti: La Seu D’Urgell 2109, ff. 164ra-167rb [inc. Quia multa et diversa tempora circa ap(p)ellationes in iure statuta sunt et in hac l..ii. Codicis de temporibus et reparationibus (C. 7.63.2); expl. [...] supra de iudiciis l. properandum (C. 3.1.13) et notatur supra de litis contestatione Auth. offeratur (Nov. $53=$ Coll. V. tit. 6, post C. 3.9.1, de litis contestatione. l. unica). Dominus Nycholaus de Mactarellis]; Madrid, Biblioteca Nacional, 823, ff. 158rb-vb [inc. C. de temporibus appellationum 1. ii (C. 7.63.2). Quia multa tempora et diversa circa appellationes in iure statuta (ripetuto) sunt; mutila della parte finale]. Sul primo dei due manoscritti si veda GARCíA Y GARCía, Iter hispanicum, n. 84. 2109, p. 112; Catálogo de los manuscritos jurídicos de la biblioteca capitular de la Seu d'Urgell, n. 2109, p. 357 s. Sul secondo si veda sopra, cap. II, nota 55.

128 Bellomo, I fatti e il diritto, p. 377 ss. e p. 379: si tratta delle quaestiones presenti ai ff. $280 \mathrm{va}-281 \mathrm{rb}$ «Statutum est in civitate Padue quod quilibet iudex teneatur sententias ferre cum consilio sapientum non suspectorum»; ff. 281rb-282va «Statutum est in civitate quod nullus contractus et nulla obligatio fiat cum minore sine auctoritate tutoris»; ff. 282va-283ra «Duo fratres a patre erede instituti».

129 ID., I fatti e il diritto, p. 380 ss., con riferimento alle quaestiones «Quidam accusabatur de aliquo crimine ex quo per legem debebat imponi pena corporalis, set per legem municipalem imponitur peccuniaria»e «Quidam accusabatur de quodam delicto et pendente accusatione mortuus est accusator», che viene risolta affermando la necessità di considerare estinta l'accusa e l'invalidità di tutti gli atti 
Il secondo codice vaticano (Chigi E.VIII.245) pare costruito con criteri fortemente analoghi al codice A.29: nella prima parte appaiono riunite quaestiones di noti giuristi attivi fra il terzo e il quinto decennio del secolo XIV, ossia gli anni in cui si formava il codice, largamente riferibili alla generazione di maestri bolognesi che ha avuto in Iacopo Bottrigari l'esponente di maggior spicco, mentre nuclei successivi ospitano quelle riferite a giuristi sempre attivi a Bologna sia nell'ultimo trentennio del Duecento, sia nei primi decenni del secolo successivo. Altre masse, invece, collocate ai fogli 75va-88ra, 99rb-111ra e $125 \mathrm{ra}-132 \mathrm{vb}$, contengono testi che, salvo poche eccezioni, sono di origine padovana oppure hanno avuto occasione di essere utilizzati a Padova, dei quali Mattarelli è frequente autore accanto a maestri di fama quali, tra i maggiori, Riccando Malombra, Tommaso di Piperata, Oldrado da Lodi, Iacopo Bottrigari, Uberto da Cremona, Bartolomeo Brancati, Vianisio dei Passipoveri, Pietro dei Cerniti e Iacopo Belvisi ${ }^{130}$. Seguono altre sequenze di quaestiones dovute sempre in

conseguenti, e pertanto anche l'annullamento della condanna già pronunciata dal giudice ignaro della morte dell'accusatore. Le due quaestiones sono trascritte in ASP A.29, ff. 314va-b e 314vb; si veda ID., Quaestiones in iure civili disputatae, n. 392 e n. 393, p. 199.

130 Per la descrizione del codice chigiano si rinvia a ID., Due 'libri magni quaestionum disputatarum', p. 255 ss.; ID., Giuristi cremonesi e scuole padovane, p. 137 ss.; ID., I fatti e il diritto, p. 176 ss. e p. 181 ss. per le masse di testi riferibili per gran parte ad autori collegati allo Studio padovano. L'analisi codicologica del manoscritto si deve a Livia Martinoli in Bellomo, Quaestiones in iure civili disputatae, Appendice E, pp. 763-771. La massa testuale che inizia a f. 125ra appare più omogenea nella prima parte che termina a f. $132 \mathrm{vb}$, mentre risulta più composita in una seconda parte che si conclude a f. 141rb, ove si alternano quaestiones, consilia e una lettura-commento di Dino del Mugello in tema di emancipazione. La sequenza delle quaestiones di Mattarelli nel codice chigiano, nel complesso assai meno numerose di quelle contenute in ASP A.29, è la seguente: ff. 75va-76rb «Quidam possidebat fundum»; ff. 76rb-78rb «Hec quaestio fuit Padue de facto. Communis consilium agebat contra quendam et dicebat eum teneri subire honera et factiones dicti communis [...]»; ff. 108vb-109rb «Quidam inculpatus de homicidio secundum formam legis municipalis bannitus est»; ff. 129ra-vb «Statutum est in civitate quod filia familias decedente matrimonio liberis extantibus»; ff. 129vb-130va «Quidam veniens ad penitentiam confexus fuit coram vicario sui episcopi»; ff. 132rb$\mathrm{vb}$ «Quidam fecit testamentum in quo instituit extraneum quendam heredem»; ff. 140va-141 rb «Quidam filios non habens fecit testamentum in quo talem fecit institutionem et substitutionem» (edita in parte in ID., Giuristi cremonesi e scuole padovane, p. 161 s., con osservazioni sulla struttura e sulla forma a p. 142, nota 23); f. 251rb-va «Iohannes ex parte una, Martinus ex altera fecerunt inter se pacem» (edita 
larghissima parte a giuristi bolognesi e padovani ma caratterizzate da differenti criteri aggregativi; tra esse anche molte di ambito canonistico disputate lungo la prima metà del Trecento, ove in forma del tutto sporadica compare ancora il nome di Mattarelli ${ }^{131}$.

La presenza di scritti del maestro modenese è ugualmente attestata, per quanto in misura quantitativamente modesta, all'interno di un terzo codice conservato presso la Biblioteca Nazionale di Roma, ben conosciuto anche per contenere alcuni frammenti di libri magni di questioni disputate e mostrare una larga corrispondenza con i testi presenti nei due codici vaticani sopra citati ${ }^{132}$. Il codice della Nazionale, di formazione per gran parte bolognese e integrato da un limitato gruppo di opere di giuristi attivi in altre sedi come Padova, Perugia o Napoli, raccoglie una massa cospicua di testi nella forma prevalente di consilia, quaestiones anche disputatae, trattati e parti di lecturae collocabili tra la metà del secolo XIII e il secondo decennio del successivo, con l'aggiunta iniziale di alcune opere bartoliane e le tracce di interventi di sistemazione complessiva compiuti da un anonimo giurista tre-quattrocentesco attivo in area umbra, forse direttamente a Perugia ${ }^{133}$.

A differenza delle serie di quaestiones incorporate nei due precedenti codici vaticani, in quest'ultimo sono riportate, di Mattarelli, due

in parte in ID., Giuristi cremonesi e scuole padovane, p. 159 s.); f. 251va-b «Quidam habens duos filios, unum ex primo matrimonio alterum ex secundo, primum emancipavit» (edita in parte in ID., Giuristi cremonesi e scuole padovane, p. 169). Si veda ID., Quaestiones in iure civili disputatae, n. 91, p. 254, n. 92, p. 254, n. 146, p. 276 , n. 182, p. 292 s., 183, p. 293, n. 186, p. 294, n. 203, p. 302, n. 396, p. 378.

131 ID., I fatti e il diritto, pp. 192 ss. e 204 ss. Nel medesimo codice vaticano si rileva anche la presenza a f. 58ra-vb della serie di glossae contrariae relative agli apparati accursiani composte da Dino del Mugello: vedi ID., Glossae contrariae di Cino da Pistoia, in part. p. 433 ss., e anche sopra, cap. II, nota 14.

$132 \mathrm{Su}$ tale aspetto, in particolare, MarTinoLI, Quattro frammenti di 'Libri magni quaestionum disputatarum'. Si riscontra una larga corrispondenza fra i testi riuniti nel manoscritto Chigi E.VIII.245 e due masse di quaestiones che, con analoghi contenuti ma con differenze nel numero e nella sequenza, sono presenti nel codice Archivio S. Pietro, A.29 e nel Vittorio Emanuele 1511: Bецlomo, I fatti e il diritto, p. 176 ss. Si veda anche Vallerani, Il diritto in questione, p. 17.

133 Martinoli Santini-Peruzzi, Catalogo delle opere giuridiche, in specie pp. 225 s., 275, 277 per i riferimenti ai testi di Mattarelli ivi contenuti; Peruzzi, Da un manoscritto miscellaneo ad una raccolta di «quaestiones», in part. p. 211 s.; MARTINOLI, Quattro frammenti di 'Libri magni quaestionum disputatarum', in part. p. $273 \mathrm{~s}$. Si veda pure la descrizione analitica del codice, a cura della stessa Livia Martinoli, alla URL <http://manus.iccu.sbn.it//opac_SchedaScheda.php?ID=69682>. 
repetitiones - o frammenti di lecturae - a leggi incluse nel primo libro del Codice e nel primo libro del Digesto ${ }^{134}$. La seconda tra queste, in particolare, ha una discreta circolazione come testo autonomo anche con il titolo Tractatus de consuetudine et iure non scripto ${ }^{135}$, che sembra qualificare una particolare sensibilità del giurista nei confronti di una delle componenti fondamentali del ius proprium anche di matrice comunale e ne attesta, tramite la sua diffusione in vari esemplari, una significativa risonanza nell'ambito della cultura giuridica entro l'orizzonte dei secoli XIV-XV, cui appartengono i manoscritti che li contengono.

Il periodo compreso fra Tre e Quattrocento corrisponde anche al limite cronologico cui giunge l'utilizzo, da parte della dottrina giuridica, di opinioni e allegazioni estratte dalle opere riconducibili a Mattarelli, che arrivano comunque a toccare quell'orizzonte temporale ormai affievolite dalla ripetizione entro sequele di richiami in cui vengono sgranati i nomi di vari maestri di consolidata fama nel panorama della dottrina giuridica trecentesca. Ne è conferma Giovanni da Imola, il quale, anche se mostra di conoscere una disputatio del giurista modenese in materia di validità dei testamenti ${ }^{136}$ e una sua quaestio ispirata a materia statutaria ${ }^{137}$, in molti casi ne rammenta l'opinione variamente riferita da Giovanni d'Andrea ${ }^{138}$, Cino ${ }^{139}$, Bartolo ${ }^{140}$ e so-

134 Ad C. de legibus et constitutionibus, l. leges et constitutiones (C. 1.14.7) (f. $184 \mathrm{va}$ ), che segue analoga opera di Iacopo d'Arena sempre a f. 184va ed è interpretabile come frammento di lectura per viam addictionum: Bellomo, Quaestiones in iure civili disputatae, n. 210, p. 479. Ad D. de legibus et senatusconsultis, 1. de quibus (D. 1.3.32) (ff. 180va-182ra), che nell'indice analitico a f. $188 \mathrm{vb}$ è indicata come Tractatus consuetudinis per ny(colaum) de mat(tarellis). Si veda sopra, cap. II, nota 59.

135 Tübingen Mc 299, f. 43ra: «Incipit tractatus de consuetudine et iure non scripto per dominum Nicolaum de Mattarellis».

136 Si veda di seguito, par. 7.

137 Una quaestio sviluppata pure da Iacopo Belvisi, dal quale potrebbe averla tratta Giovanni. IOANNIS AB ImOLA, In secundum Decretalium commentaria, f. 189vb, n. 40: «[...] post Nicola. de Mata. et Iaco. de Belvi. ubi tangunt questionem quid si statuto cavetur, quod filiusfa. non possit obligari sine expressa licentia patris».

138 IOANNIS DE IMOLA, In primam Infortiati partem, f. 96va, n. 9; IOANNIS AB Imola, In secundum Decretalium commentaria, f. $273 \mathrm{vb}$, n. 11.

139 IoAnNis aв Imola, In secundum Decretalium commentaria, f. 71rb, n. 5; f. 71va, n. 7; f. $273 \mathrm{vb}$, n. 11.

140 IOANNIS DE IMOLA, In secundam Digesti novi partem, f. 138va, n. 7; IoANNIS AB IMOLA, In secundum Decretalium commentaria, f. 95vb, n. 10; f. $131 \mathrm{ra}, \mathrm{n}$. 21; f. 134 va, n. 24. 
prattutto Baldo ${ }^{141}$ oppure incastonata in modo seriale entro sequenze più o meno ricche di richiami concordi ai medesimi giuristi, talora variati dalla citazione di altri autorevoli colleghi ${ }^{142}$; in altri casi più sporadici la citazione di Mattarelli appare veicolata attraverso il richiamo fissato dal proprio maestro Antonio da Budrio, presente alla laurea in diritto canonico conseguita da Giovanni a Bologna il 7 agosto $1402^{143}$.

\section{Un isolato interesse canonistico}

È infine da segnalare una quaestio disputata di Mattarelli in merito a una norma formulata da papa Gregorio X concernente il divieto per gli usurai di fare testamento, rubricata da Alberico al numero 299 del proprio Liber magnus quaestionum disputatarum ${ }^{144}$, rintracciabile all'interno di uno fra $\mathrm{i}$ codici vaticani sopra ricordati ${ }^{145}$ e riecheggiata ancora da Giovanni da Imola nel commentario alla prima parte dell'Inforziato, risalente alla metà degli anni Trenta del secolo $\mathrm{XV}^{146}$. Da par-

141 IOANNIS DE IMOLA, In secundam Infortiati partem, f. 90vb, n. 3; IOANNIS AB Imola, In secundum Decretalium commentaria, f. 134va, n. 23; f. 195rb, n. 33 e n. 34; f. 195va-b, n. 34; f. 196ra, n. 34.

142 IoAnNis De Imola, In primam Infortiati partem, f. 66rb, n. 74; f. 68vb, n. 10; f. $74 \mathrm{rb}, \mathrm{n} .75$ in fine; f. 90rb, n. 7.

143 IoANnIS AB Imola, In secundum Decretalium commentaria, f. 130vb, n. 21; f. 274rb, n. 11; f. 274va, n. 11. Per il magistero di Antonio da Budrio: PADOvANI, Giovanni Nicoletti, p. 1016; ID., Giovanni da Imola, pp. 155, 157 s.; ID., Dall'alba al crepuscolo del Commento, pp. 23, 44 ss.

144 Alberico da Rosciate, Commentarium de Statutis, 1. I, q. 150, ff. 23vb24rb: «Quidam usurarius manifestus non praestita cautione fecit testamentum, demum post aliquod tempus prestitit cautionem predictam. Quaeritur an praedictum testamentum valeat». Ricordata in Romano, Le quaestiones disputatae, n. 37, p. 95 s.

145 Archivio S. Pietro, A.29, ff. 270ra-271ra. Si veda Bellomo, Quaestiones in iure civili disputatae, n. 348, p. 179 s. In merito CORTESE, Il Rinascimento giuridico, p. 65 e nota 191, seguito da LABARDI, Mattarelli Nicolò, p. 102, ritiene che la decretale pontificia non abbia trovato accoglienza nel Sextus.

146 Nel commento alla lex Filio praeterito del Digesto vecchio (D. 28.3.17) in relazione alla validità del testamento dell'usuraio non praestita cautione de restituendis usuris secundum formam ibi posita: GiovanNI DA IMOLA, In primam infortiati partem, f. 65vb, n. 71: «[...] et pro ista parte, quod testamentum reconvalescat, facit id quod tenet Nic. de Mata. in sua disputatione, ubi hoc idem tenet et pro responsione ad contraria considerabat quatuor casus, quia interdum testamentum deficit propter defectum personae testantis [...], aut deficit ratione solennitatis 
te del civilista modenese si tratta di una sporadica manifestazione del contatto con una materia anche di interesse canonistico, che non pare rinnovarsi in altri ambito della sua riflessione dottrinaria né tanto meno in riferimento a problematiche conciliari, che diverranno maggiormente scottanti soltanto nei primi decenni del Quattrocento ${ }^{147}$.

La disputa trae spunto dal dettato di una constitutio attribuita a papa Gregorio X poi confluita nel Sextus ${ }^{148}$ concentrata sul divieto, per l'usuraio, di fare testamento a meno che non abbia restituito il denaro guadagnato in modo illecito o non abbia prestato idonea cauzio-

[...], aut deficit ratione personae instituti [...], aut deficit ex alio accidenti extrinseco [...]». PAdovani, Dall'alba al crepuscolo del commento, p. $51 \mathrm{~s}$. per la redazione dell'opera «nella versione divulgata dalle stampe».

147 Non si può pensare a un consilum «di Niccolò Mattarelli in difesa delle idee conciliariste, alla vigilia del consilio di Pisa del 1409», come dichiarato da CoRTESE, Canetoli Giovanni, p. 410, e ancora ID., Paolo di (da) Castro, p. 1507. In realtà, in materia conciliare la raccolta di consilia di Paolo di Castro ne incorpora uno di Antonio da Budrio (n. 419) seguito da un altro del bolognese Matteo Mattesillani (n. 420), datato 11 ottobre 1407 e sottoscritto pure da un altro giurista bolognese, Giovanni Canetoli. Il testo è chiuso da una nota esplicativa del curatore dell'opera, Nicolaus Michaelis, tesa a precisare che «Haec facti species cum suo consil. in antiquo Codice reperitur post consi. 418 inter consi. Praemitto, quod hic est sub illo nu. et consil. Viso puncto, quod est hic in ordine 419, tamen quia materiae illorum consiliorum sunt connexae invicem, et pariter materia cons. 420 ne fiat confusio, hoc loco relinquimus praemissa monitione. N.M.» (Paolus De CASTRo, Consiliorum sive responsorum [...] volumen primum, n. 418, ff. 213rb-214ra; n. 419, ff. 214ra215ra; n. 420, f. 215ra-va, a f. 215va per la citazione).

148 Come ricorda con precisione lo stesso Alberico, secondo il quale «olim extravaganti, hodie de usur. c. 2, lib. 6» (= VI, 1. V, tit. V De usuris, cap. 2). La norma discende da uno dei canoni approvati in occasione del XIV concilio ecumenico di Lione, convocato da Gregorio X per il I maggio 1274 con l'obiettivo prioritario di discutere della riforma della disciplina ecclesiastica, dei rapporti con la Chiesa orientale e del soccorso alla Terrasanta: Hefele-Leclerco, Histoire des conciles, $\mathrm{VI} / 1$, p. 153 ss. e p. 181 ss. per i canoni approvati nel corso delle sue varie sessioni, tra i quali si contano i cc. 26 e 27 relativi agli usurai; nel c. 27 è affermata l'invalidità del testamento dell'usuraio nel caso egli non abbia provveduto a restituire il denaro illecitamente guadagnato o non abbia rilasciato idonea cauzione. Non appunta l'attenzione sulla normativa canonica formulata da Gregorio X in materia testamentaria, come neppure sui canoni del Concilio lionese, il succinto profilo di Dumas, Testament. A papa Gregorio, d'altronde, non si può riconoscere un contributo particolare e caratterizzante nella definizione della normativa canonica in materia testamentaria, che si è piuttosto avvalsa dei più incisivi interventi di Alessandro III, Innocenzo III e IV, Gregorio IX e Bonifacio VIII: Merzbacher, Das Testamentsrecht des Corpus Iuris Canonici, in part. p. 291 s. Alcune indicazioni circa le prescrizioni canonistiche in materia di testamenti entro l'orizzonte del secolo XII sono fornite da SHEeHan, The Will in Medieval England, p. 120 ss. 
ne per la sua restituzione. Scaturisce quindi il problema della validità del testamento nel caso in cui la cauzione sia prestata in tempi successivi alla sua scrittura, che Alberico, sulla scia del maestro modenese ${ }^{149}$, risolve con spirito fortemente pratico, attento più alla tutela della volontà soggettiva piuttosto che alle implicazioni morali e spirituali della condizione di usuraio, tramite una catena di allegazioni e di casus a base unicamente romanistica riconoscendo piena validità alla disposizione testamentaria: la norma canonica non ne proibisce formalmente la redazione in via assoluta, ma la subordina alla prestazione di una garanzia che per i due giuristi può essere formata anche in seguito, quia tantum operatur ex post facto cautio, quantum si ab initio praestita foret. Essa non attiene alla sostanza del testamento, ma conserva il carattere di un quidam actus remotus ab ipso tale da assicurarne l'efficacia in qualsiasi momento essa venga prestata.

A Mattarelli, seguito in ciò dal giurista bergamasco, interessa maggiormente riflettere sulle disposizioni di ultima volontà e sulla latitudine della loro efficacia piuttosto che sulla specifica problematica dell'usura e della sua declinazione sub specie iuris, materia invece particolarmente sensibile per la Chiesa e per il diritto canonico ${ }^{150}$ ma presente anche nell'orizzonte dei civilisti, che pur si muovono su una trama normativa del diritto civile giustinianeo estremamente frammentata in materia ${ }^{151}$.

Per quanto nella sua ricca collana di quaestiones Alberico si dimostri ben attrezzato nella conoscenza della dottrina canonistica e abbia familiarità con gli apparati anche al corpus iuris canonici ${ }^{152}$, egli recupera la quaestio di Mattarelli nella sua prospettiva tutta ci-

149 Un caso specifico, evidenziato da STORTI Storchi, Prassi, dottrina ed esperienza legislativa, p. 452, in cui il giurista tende «ad aderire al risultato della elaborazione scientifica di un singolo maestro solo se confortato dal consenso della dottrina prevalente o applicato nella vita giudiziaria».

150 Sul tema della restituzione del denaro lucrato in forme usurarie si veda TodeschinI, I mercanti e il tempio, in part. cap. IV (Restituire: indennizzare) per i riferimenti alla riflessione canonistica soprattutto in relazione al Decretum Gratiani e alla normativa canonica prodotta entro la metà del Duecento.

151 Un approfondimento sulla riflessione della Scuola del Commento tra Cino e Baldo sul tema complessivo dell'usura e degli equilibri, in materia, tra istanze canonistiche e civilistiche si deve in anni recenti a QuAGLIONI, Standum canonistis?

152 Storti Storchi, Prassi, dottrina ed esperienza legislativa, p. 440; EAD., Alberico da Rosciate, p. 21. 
vilistica. Soltanto in chiusura ricorda il parere opposto di Giovanni d'Andrea, radicalmente contrario, in consonanza con la rigida norma canonica, a riconoscere validità al testamento dell'usurario sin dal momento della sua originaria formazione, come pure ad accettare che la cauzione richiesta iure canonico venga prestata dagli eredi del testatore usuraio ${ }^{153}$.

\section{De hac materia summam prolixam formavit}

Nel ventaglio di testi attribuiti a Niccolò Mattarelli una posizione non marginale è occupata dall'opera modernamente nota come Tractatus super instrumentis, leggibile all'interno di un unico manoscritto vaticano di provenienza bobbiese ${ }^{154}$, già riconosciuto verso la fine del XIX secolo ${ }^{155}$ e contenente una vasta silloge di opere giuridiche formata da repetitiones, quaestiones, tractatus e consilia

153 Con riferimento alla gl. Aliter facta, ad VI, l. V, tit. V, in fi., e al commento ribadito in GIOVAnNI D'ANDrEa, Novella in Sextum, l. IV, tit. de usuris, in fi., ove si ricorda la solemnis disputatio di Mattarelli sull'argomento.

154 Con riferimento al Vat. Lat. 5773, ceduto nel 1618 dai monaci di Bobbio alla Biblioteca Vaticana su richiesta di papa Paolo V all'interno di un manipolo di una trentina di manoscritti: Mercati, M. Tulli Ciceronis De re publica, p. 139 ss. Anche FERRARI, $I l$ codex Muratorianus, p. 11, dichiara la provenienza dal monastero di S. Colombano di Bobbio del codice in questione e il possesso del medesimo, in un periodo non definito, da parte della famiglia de Georgis assieme ad altri manoscritti a contenuto anche giuridico poi confluiti nella Biblioteca Nazionale di Torino (attuali F.IV.10, F.IV.11, F.IV.13). Il manoscritto cartaceo F.IV.13, in particolare, contiene le Tragedie di Seneca e la sua copia viene conclusa a Savona il primo luglio 1411 dal giurisperito bobbiese Antonio de Georgis: PetтI Balbi, Cultura e potere a Genova, p. 254. Su questa famiglia di origini pavesi, considerata tra le più in vista nel panorama sociale del piccolo centro appenninico tra Cinque e Seicento anche se nessuno dei suoi membri vi ricoprì mai la carica di podestà (FIORI, I podestà di Bobbio), si veda il profilo riassuntivo di ID., Antiche famiglie di Piacenza, p. 484 s., e anche ID., Le istituzioni ecclesiastiche minori bobbiesi, p. 322 e p. 337, nota 31, per l'epigrafe funeraria dell'illustris iuris consultus Giannotto Giorgi, defunto nel 1462 e tumulato nella cattedrale di Bobbio. Un breve accenno all'appartenenza dei Giorgi alla nobiltà bobbiese riconosciuta tramite il possesso di singoli stemmi famigliari già in ID., Osservazioni sulle famiglie nobili di Bobbio, p. 24.

155 Seebass, Handschriften von Bobbio, p. 3 s.: mancante di tracce evidenti di appartenenza alla biblioteca del monastero di S. Colombano di Bobbio, il codice vi può essere giunto dopo la chiusura dell'inventario redatto nel 1461 e pubblicato in Cipolla, Codici bobbiesi, pp. 15-18. Potrebbe derivare da un lascito disposto da 
risalenti ai secoli XIV-XV ${ }^{156}$. Il codice è utilizzato presso lo Studio pavese verso la fine del Trecento e certamente nell'agosto del 1395, come attesta una annotazione a f. $11 \mathrm{va}$ in cui riecheggia anche il nome di Baldo, e conserva pure a f. $37 \mathrm{v}$ una nota d'uso vergata da un allievo di Raffaele Fulgosio, che insegna diritto civile a Pavia fino al $13999^{157}$.

Il testo di Mattarelli che vi è riportato è stato oggetto di una recente e meritevole trascrizione che ha privilegiato criteri a base essenzialmente diplomatistica lasciando, di conseguenza, ampio spazio alla sua integrazione tramite un commento storico-giuridico, che in questa sede sarà tuttavia circoscritto a due soli profili comunque non irrilevanti.

L'opera nasce, assai verosimilmente, in ambito scolastico come repetitio al Titolo 21 (De fide instrumentorum) del IV libro del Codice e si presenta come un tentativo di fornire un inquadramento teorico e sistematico alla materia delle scritture utilizzabili a fini probatori redatte dai notai nei diversi ambiti dell'attività professionale e rispetto alla molteplicità delle esigenze e delle funzioni sollecitate dall'ormai matura civiltà comunale nei decenni di passaggio tra Due e Trecento. Nel testo, che non è privo di una certa originalità quanto a profilo tematico e impianto espositivo, sono considerate anche altre categorie di scritture che possono acquisire rilevanza in sede processuale, come quelle prodotte dagli argentarii (banchieri) e dai mercanti, in ciò mostrando un interesse calato in forme robuste nel

giurista Giannotto, figlio di Antonio Giorgi, che muore l'anno successivo, nel 1462 (cfr. nota precedente).

156 Vat. Lat. 5773, ff. 47va-50vb. Il codice è censito in Sella, Appunti su alcuni Codici Giuridici Vaticani, p. 383. La presenza in esso di opere di Mattarelli era già segnalata in MonTFAUCON, Bibliotheca bibliothecarum, I, p. 141. In tempi recenti ne dà notizia, in forma cursoria, NicolaI, Lineamenti di diplomatica generale, p. 85, nota 174; EAD., Lezioni di diplomatica generale, p. 88, nota 133. Considerata la varietà dei numerosi testi in esso riuniti, che, a parte vari anonimi, attestano la produzione di un gran numero di giuristi attivi fra Tre e Quattrocento, il manoscritto è utilizzato anche da Baumgärtner, Quidam presbiter beneficialis, $p$. 222 ss., per l'edizione di un consilium di Martino Garato da Lodi; da MARI, Un consiglio collegiale, p. 115 , nota 408 , per la sottoscrizione del giurista pavese Torberto Torti a un consilium ancora di Martino Garato; da MüLLER, Signorolus de Homodeis, p. 230, per la segnalazione di un consilium del giurista milanese contenuto ai ff. 174ra-175rb; da Peruzzi, Prime note, p. 885 s. per la copia di un consilium di Francesco Tigrini.

157 Murano, Raffaele Fulgosio, pp. 145 e 147. 
mondo della prassi e decisamente precoce rispetto a quello palesato dalla posteriore dottrina giuridica trecentesca ${ }^{158}$.

$\mathrm{Al}$ centro dell'attenzione del giurista è soprattutto l'atto notarile in quanto strumento di prova documentale, esaminato sotto il profilo di tre categorie connesse ad altrettante fasi dell'attività del notaio: una prima, di notevole sviluppo, dedicata alla confectio del documento di prova considerato nelle sue varie tipologie formali e funzionali; una seconda, brevissima, riservata all'editio e alla traditio del documento e alla sua conseguente produzione in sede processuale; una terza, infine, inerente la scrittura probatoria considerata vacua, ossia invalida, e pertanto inefficace a provare le ragioni per cui è stata prodotta in giudizio ${ }^{159}$.

Destino peculiare del testo di Mattarelli è stato quello di essere in larga misura riprodotto da Giovanni d'Andrea nelle Additiones allo Speculum di Guglielmo Durante, a margine del corposo Titolo De instrumentorum editione e in specie nell'ampia glossa alla rubrica iniziale $^{160}$. L'autorevole canonista, collega per un triennio di Matta-

158 Come osserva Pecorella, Fides pro se, p. 155, in rapporto soprattutto alle scritture mercantili.

159 Una prima descrizione dell'articolazione interna dell'opera è fornita da Macino, Contributo alla storia della trasmissione del Tractatus super instrumentis, p. 258 ss.

160 Guglielmo Durante, Speculum iudiciale, II, II, De instrumentorum editione, Rubrica, t. 1, p. 629 ss. Non vi è dubbio che la summam prolixam che Giovanni esplicitamente attribuisce a Mattarelli circa scripturam probatoriam, quae dicitur intrumenta sia da ravvisare nell'opera conservata nel codice Vaticano latino 5773, nonostante alcuni dubbi avanzati da Pecorella, Fides pro se, nota 72, p. 159 s., in merito soprattutto al valore dell'aggettivo prolixus (con significato originario di 'ampio', 'esteso') rispetto al ritmo e allo sviluppo effettivo del testo composto dal giurista modenese. Medesima qualificazione Giovanni usa nei confronti del prolixum tractatum sugli esecutori testamentari, articolato in 135 quaestiones, composto nel 1263 dall'avvocato e giudice marsigliese Jean Blanc (GuGLIELMo DuRANTE, Speculum iudiciale, II, II, De instrumentorum editione, t. 1, p. 691), allievo a Modena negli anni Trenta del Duecento di Uberto di Bonaccorso, Uberto da Bobbio e Omobono da Cremona e autore pure di una Summa super usibus feudorum intorno alla metà del secolo: CAILlEMER, Les débuts de la science du droit en Provence, p. 772 ss. e p. 784 ss. per l'opera di Jean Blanc ripresa pressoché integralmente da Giovanni d'Andrea al pari di quella di Mattarelli; Cortese, Scienza di giudici e scienza di professori, p. 142 s.; GIORDANENGo, Jean Blanc feudiste de Marseille; ID., Les feudistes, pp. 79, 108 s.; ID., Le droit féodal dans les pays de droit écrit, pp. 133 s., 141 ss.; ID., Blanc (Blancus) Jean. Pure Jean Blanc è tra i numerosi giuristi che, a partire da Pillio, si sono interessati alla materia feudale ricordati da Baldo nella sua Lectura feudorum: DANUSSO, Ricerche sulla «Lectura feudorum», p. 51. Tornando 
relli a Padova tra 1306 e 1309, potrebbe avere avuto facile e immediato accesso al testo predisposto da quest'ultimo e, pur seguendone da vicino impostazione e contenuti, approfonditi anche sulla base di interessi comuni nel periodo del magistero patavino ${ }^{161}$, si sarebbe impegnato a darne una versione filtrata attraverso «una modalità espressiva più accademica e scolastica» basata, oltre che sul timbro fortemente didascalico, su una formale aderenza alla tradizione romanistica sia dal punto di vista linguistico ed espressivo, sia da quello delle opzioni in merito a contenuti, figure ed elementi strettamente pertinenti a quest'ultima ${ }^{162}$.

Anche Baldo mostra di avvalersi ampiamente dell'opera monografica di Mattarelli dando così un forte contributo, dopo Giovanni d'Andrea, alla sua conoscenza indiretta nel mondo della posteriore dottrina giuridica ma favorendone, nel contempo, la sostanziale scomparsa dalla circolazione sia manoscritta, sia dal circuito delle successive riproduzioni a stampa. Baldo utilizza il trattato del doctor copiosus et subtilis ${ }^{163}$ non tanto nella giovanile operetta dedicata alla figura e alle funzioni dei tabelliones ${ }^{164}$, ma soprattutto all'interno del commentario al quarto libro del Codice, che egli pubblica in seguito a Padova, dove Mattarelli aveva insegnato per molti anni e dove Baldo, verosimilmente, ne aveva preso diretta visione, in atti-

brevemente a Mattarelli, anche Baldo ne ricorda, almeno in un passo, la prolixitas verborum: BALDI UBALDI, In quartum et quintum codicis libros commentaria, $\mathrm{f}$. 125ra, n. 13 (ad C. 4.50.6).

161 Su tale argomento, come dichiara lo stesso Giovanni, i due maestri ebbero modo di fornire assieme una consulenza comune: «[...] quia in quadam quaestione, quam circa materiam ipse et ego consulere habebamus, Paduae satis illam revolvimus, ideo hic illam ponam, quam brevius potero, prosequendam» (GuGLIELmo Durante, Speculum iudiciale, II, II, De instrumentorum editione, Rubrica, t. 1, p. 629).

162 Macino, Contributo alla storia della trasmissione del Tractatus super instrumentis, p. 262 ss.

163 Così è definito Mattarelli in BALDI UBALDI, In quartum et quintum codicis libros commentaria, f. 36rv, n. 3 (ad C. 4.19.3).

164 Valentini, Il «Tractatus de tabellionibus» di Baldo degli Ubaldi: si tratta di un'opera composta a Perugia verso la metà del Trecento e di impianto fortemente compilativo, costituita da una collana di 25 quaestiones, molte delle quali si esauriscono nella esposizione delle contrastanti opinioni di autorevoli maestri, sia civilisti che canonisti, sul tema di ciascuna, con forte preferenza per allegazioni tratte dagli scritti di Sinibaldo Fieschi, Guillaume de Cun, Bartolo, Guglielmo Durante, Dino, Jacopo Belvisi, Jacopo Bottrigari, Cino e Ranieri da Forlì. 
nenza a una delle sedes materiae privilegiate per l'esame della documentazione scritta e dell'attività del notaio sub specie iuris ${ }^{165}$.

Sebbene Mattarelli sviluppi la trattazione sulla base di una solida griglia di argomentazioni e allegazioni radicate esclusivamente del diritto romano e nei modelli interpretativi da questo assicurati, senza prestare attenzione alla dottrina giuridica a eccezione del consolidato appiglio alla Glossa ${ }^{166}$ e con ricorso del tutto marginale

165 Esplicito il debito con l'opera del predecessore dichiarato in BALDI UBALDI, In quartum et quintum codicis libros commentaria, IV, De fide instrumentorum, Rubrica, f. 55vb, n. 1. «[...] ut autem de his aliqua utiliter dicam, summatim pertractabo ea quae tractat hic Nic. de Mat.». Fittissime poi le allegazioni del maestro modenese nello sviluppo del commento al medesimo Titolo del Codice giustinianeo, in specie alla stessa lex "Contractus", che permettono di verificare la stretta aderenza di Baldo alla struttura e ai contenuti del trattato di Mattarelli. Il fatto che il testo di Mattarelli sia in gran parte confluito nell'opera di Giovanni d'Andrea e in quella posteriore di Baldo è sottolineato già da Pecorella, Studi sul notariato a Piacenza, pp. 3 s., 115, che costituisce l'unico studio - a mia conoscenza - in cui viene diffusamente esaminata questa specifica opera del giurista modenese, artefice del primo saggio che ex professo abbia sviluppato «con una certa completezza l'esame dei problemi che si agitavano nel mondo dei giuristi con riferimento all'attività notarile» (ibidem, p. 115).

166 Rinvii alla Glossa emergono in varie sezioni dell'opera: Vat. Lat. 5773, ff. 48ra, 48rb, 48vb, 49va, 50rb; cfr. MatTarell, Tractatus super instrumentis, pp. 33, 37, 39, 45, 47, 60, 69. Diversamente si rilevano soltanto due sporadiche allegazioni dottrinarie relative ad Angelus e a Cynum (Vat. Lat. 5773, ff. 48rb, 48va; cfr. ID., Tractatus super instrumentis, pp. 37, 44), che tuttavia sembrano frutto dello scivolamento all'interno del testo, giunto in copia quattrocentesca, di riferimenti originariamente contenuti in note marginali. In alcune di quelle ancora presenti nel manoscritto vaticano compare infatti il richiamo a passi tratti dalla Lectura codicis di Angelo degli Ubaldi, che ovviamente non poteva essere nota a Mattarelli (ID., Tractatus super instrumentis, pp. 38 nota a, 42 nota a, 58 nota b, 68 nota a, 75 nota b, 78 nota b). Al contrario, Angelo conosce, almeno in parte, l'opera di Mattarelli (segnatamente la sua operetta de consuetudine et iure non scripto, per cui vedi sopra paragrafo 6), come testimonia un'allegazione contenuta nella repetitio in Inst. 1.2.9 del maestro perugino all'interno del codice Vaticano, Ottoboniano latino 1726 (Cortese, La norma giuridica, II, p. 106, nota 14). Analogamente, è Cino a includere riferimenti all'opera del giurista modenese all'interno della propria Lectura codicis, e non, ovviamente, il contrario. Al richiamo a Cino presente all'interno del testo di Mattarelli si affianca, sul margine della medesima carta, una nota nella quale, circa la possibilità per un notaio di facere instrumentum traendolo ex protocholo di un altro notaio, si richiama l'opinione del giurista pistoiese nella parte finale del commento alla lex "Contractus": Cino Da Pistoia, Lectura super Codice, in lib. IV, tit. XXI De fide instrumentorum, f. 224va, n. 14 (C. 4.21.17). 
a fonti canonistiche e citazioni scritturali ${ }^{167}$, dall'esame della vasta materia documentaria emergono profili strettamente connessi al vivo tessuto dei bisogni materiali del proprio tempo ${ }^{168}$.

Basti considerare le problematiche legate al riconoscimento del valore probatorio delle scritture mercantili o alla legittimazione delle imbreviature notarili quali mezzi di prova al pari degli intrumenta redatti in mundum. In entrambi i casi il giurista, che procede nell'esposizione dei molteplici profili ancorandoli a una sequenza gerarchica scandita attraverso i collaudati strumenti della distinctio e della quaestio, non offre soluzioni d'avanguardia, ma sviluppa una esposizione completa ed equilibrata degli elementi caratteristici dei due ambiti segnando, con le sue scelte, una tappa importante nella traiettoria che segna il mutamento di valore delle due tipologie di scritture e il loro autonomo riconoscimento in sede giudiziaria.

\subsection{Le imbreviature dei notai}

Nel lessico di Mattarelli le scritture prodotte dai tabellioni-notai, rispecchiando la netta contrapposizione prevista dal diritto romano ${ }^{169}$, si dividono in due precise categorie: il mundum, ovvero la scrit-

167 Si osserva la rapsodica allegazione di capitoli del Liber Extra estratti dai Titoli De probationibus e De fide instrumentorum del II libro (c. Quoniam contra $=$ X. 2.19.11; c. Cum tabellio = X. 2.22.15; c. Ex litteris = X. 2.19.3; c. Post cessionem $=$ X. 2.19.7; c. Cum dilectus $=$ X. 2.22.9; c. Inter dilectos = X. 2.22.6; c. Scripta $=$ X. 2.22.2; cfr. MatTARelli, Tractatus super instrumentis, pp. 24, 26, 49, 73, 76) e di due soli passi scritturali (ibidem, p. 39 s.).

168 Un unico accenno alla diretta esperienza personale emerge nel riferimento all'incerta decifrazione delle imbreviature nei casi in cui queste presentino troncamenti e abbreviazioni che rispondono a criteri unicamente soggettivi dell'estensore: Vat. Lat. 5773, f. 49va («Sed tunc potest dubitari quid si imbreviatura habeat detruncaciones in oracionibus, ut quia dicit "Ticus promossit et cetera", item "renunciavit et cetera" et symilia vel forte habe $<a>t$ notas et signa aliquid significancia secundum intencionem scribentis, non tamen secundum ordinationem litterarum, ut quia ponebat duo "cc" pro uno "a", ut iam vidi [...]»); cfr. MatTAReLLi, Tractatus super instrumentis, p. 49. Si aggiunga un isolato richiamo, circa le scritture probatorie redatte dai banchieri (argentarii), al «servo uxori mee [...], qui non erat argentarius [...]»: Vat. Lat. 5773, f. 49va; cfr. ID., Tractatus super instrumentis, p. 60.

169 Crescenzi, La rappresentazione dell'evento giuridico, p. 86 ss. I principali provvedimenti normativi emanati dall'età costantiniana a quella giustinianea in materia di redazione di documenti e di attività dei tabellioni sono esaminati, con 
tura perfezionata e completata in tutte le sue parti, e l'imbreviatura que vocatur scheda, il primo abbozzo scritto dal notaio finalizzato ad appuntare gli estremi essenziali del negozio recependo le primissime volontà dei contraenti e per ciò definibile anche come protocollo ${ }^{170}$.

La scheda, ancora così formalmente identificata in base all'appiglio normativo garantito da Giustiniano tramite la lex "Contractus" (a. 528) ${ }^{171}$, viene equiparata in modo esplicito all'imbreviatura, quia ibi breviter comprehenditur intencio contrahencium, che è identificata a sua volta, secondo l'uso corrente affermatosi nella prassi e non soltanto ${ }^{172}$, nel protocollo, id est prima collatio scripturarum instrumenti sive prima scriptura instrumenti, contenente gli elementi distintivi fondamentali da ripetere nella carta destinata ad accogliere l'instrumentum mundum ${ }^{173}$. Tra quest'ultimo e l'imbreviatura/pro-

riferimento a bibliografia specifica, da Feo-Iannacci-Zuffrano, Il formulario del documento privato, par. I. Il periodo post-classico. In merito si veda anche AмELоттI, Notaio. Diritto romano, p. 555 ss.

170 Vat. Lat. 5773, f. 48rb; l'equivalenza imbreviatura-scheda è ribadita a f. 47vb; MatTaRelli, Tractatus super instrumentis, p. 41 e p. 30 rispettivamente. Differente in questo la prassi notarile e l'orientamento che cerca faticosamente di imprimere ad essa la normativa statutaria duecentesca, laddove distingue tra scritture preparatorie concise e stringate, riassuntive delle volontà delle parti e dei beni oggetto di transazione (notae, notulae, schedae, rogationes), e scritture invece complete di tutte le formule e clausole necessarie (imbreviature), pronte ad essere trasformate, quando richiesto, nell'instrumentum definitivo: TАмвА, Una corporazione per il potere, p. 181 s. Già al tempo di Ranieri, quindi almeno dagli anni Venti del secolo XIII, le rogationes erano raccolte «in quaterno vel libro rogationum dicti tabellionis»: RANIERI DA PERUgIA, Ars Notariae, rub. [LXVII] De exenplationibus scripturarum, p. 67.

171 Con riferimento specifico a C. $4.21 .17 \mathrm{pr}$. e C. 4.21.17.1. In questo titolo del Codice giustinianeo confluisce materiale normativo derivato «da una produzione legislativa che spazia dal III al VI secolo» e «tutto incentrato sulla accezione di instrumentum come atto scritto»: CRESCENZI, La rappresentazione dell'evento giuridico, p. 72 ss. e p. 95 per la citazione.

172 Anche la dottrina canonistica concorda nell'identificazione tra protocollo, nota e imbreviatura: Henrici de Segusio cardinalis Hostiensis, Summa aurea, 1. II, De fide instrumentorum, col. 659: «Item quando tabellio morte perventus est et tenor remansit in protocollo sive nota vel abbreviatura [...]». In seguito anche Durante accetta l'immediata equivalenza tra notula e protocollo: Guglielmo DuRANTE, Speculum iudiciale, II, II, De instrumentorum editione, § Instrumentum, t. 1, p. 667.

173 Vat. Lat. 5773, ff. 48rb-48va, e anche 48vb, ove si ribadisce che il protocollo est principium instrumenti. Cfr. MatTARell, Tractatus super instrumentis, pp. 41, 47. La definizione di protocollo è ormai lontana dall'originale giustinianeo richiamato nella Novella 44, capo II (= Auth. 45, Coll. IV, tit. 7, a. 536), ove si giu- 
tocollo non si devono riscontrare differenze negli elementi essenziali caratterizzanti il negozio giuridico, espressi soltanto in forma più ampia e chiara nel primo tra essi, con il rischio, in caso contrario, che l'instrumentum sia giudicato falso e adulterato ${ }^{174}$.

Un'unica oscillazione pare Mattarelli manifestare laddove identifica l'imbreviatura come secunda scriptura e il mundum come prima scriptura ${ }^{175}$; il che può interpretarsi come un riferimento alla semplice gerarchia di importanza delle due versioni, da riconoscersi in misura maggiore nell'atto perfezionato in tutte le parti, privo di abbreviazioni e di parti ceterate tipiche della scrittura preliminare

stificava nella prescrizione, per i notai costantinopolitani, di scrivere gli atti su fogli nella cui parte superiore (il protocollo) fossero preventivamente riportati il nome del comes sacrarum largitionum e la data di confezionamento della carta stessa, allo scopo di assicurarne l'autenticità e impedire falsificazioni; un obiettivo che il tabellionato bizantino, alla luce delle prescrizioni fissate anche nella Novella 47 (= Auth 48, Coll. 5, tit. 3, a. 537), assolse sviluppando, per la scrittura di tale specifica sezione dei documenti, una tecnica grafica assolutamente particolare: si veda Iannacci, Modesti, Zuffrano, La misteriosa scrittura grande dei papiri ravennati, p. 106 ss., e anche CRESCENZI, La rappresentazione dell'evento giuridico, pp. 97 ss., 118 ss., 127 ss., 145 ss., 173 ss., 184 ss., per l'analisi coordinata degli interventi legislativi fissati nelle Novelle giustinianee (Novelle 44, 47 e 73) e destinati a tracciare una disciplina organica in tema di instrumenta e tabelliones. Dall'esposizione di Mattarelli si evince che l'equivalenza tra imbreviatura e protocollo è giustificata dal fatto che quest'ultimo, a tenore dell'originaria disposizione legislativa, deve contenere inderogabilmente l'indicazione della data cronica, elemento già presente anche nella primitiva raccolta, da parte del notaio, degli estremi essenziali del negozio in base alla volontà espressa dalle parti e tale perciò da connotare, per consuetudine invalsa, l'identità complessiva della notazione preparatoria vergata dal notaio: «Et ideo consuetudo, que est optima legum interpres, induxit declarando huiusmodi protochola in quinternis tabellionum scribi, ex quibus postea intrumentum mundum trahitur quod datur contrahenti» (Vat. Lat. 5773, f. 48va; cfr. MATTARELLI, Tractatus super instrumentis, p. 42). L'equivalenza tra le diverse denominazioni della scrittura preparatoria sono pacificamente condivise da CINO DA PISTOIA, Lectura super Codice, in lib. IV, tit. XXI De fide instrumentorum, f. 223vb, n. 1 (C. 4.21.17): «Nota in primo dicto quod contractus qui initus est in scriptis non ante habet efficaciam, quam in scheda fuerit positus, quam vulgus appellat rogationem sive rogitum, que est protocollum sive imbreviatura, et ex scheda sive protocollo redactus fuerit in mundum et absolutus a partibus».

174 Vat. Lat. 5773, f. 48va; cfr. MatTARelLi, Tractatus super instrumentis, p. 42.

175 Vat. Lat. 5773, f. 47vb; cfr. MatTaRelli, Tractatus super instrumentis, p. 28 , ove la scelta dell'editore è quella di emendare il testo in base alla forma compendiata preferita da Giovanni d'Andrea in Guglielmo Durante, Speculum iudiciale, II, II, De instrumentorum editione, Rubrica, t. 1, p. 630b, snaturando tuttavia il senso dell'originaria espressione di Mattarelli. 
e corroborato dalle publicationes del notaio. Ma si può leggere pure come un'allusione ai casi in cui l'imbreviatura sia redatta successivamente al mundum, soprattutto per motivi di garanzia a fronte della possibile impugnazione di falso di quest'ultimo e della non obbligatorietà, da parte del notaio, di conservare le imbreviature; una prescrizione, quest'ultima, rispetto alla quale la prassi, disciplinata dalla normativa statutaria, aveva generalmente imboccato una direzione opposta ${ }^{176}$. È tuttavia già ben chiaro ai tempi di Odofredo e del suo allievo Salatiele, entro l'orizzonte degli anni Quaranta del secolo XIII, che è del tutto tramontato l'uso di vergare la scrittura preparatoria su una faccia della pergamena (lato pelo) e l'atto completo e perfezionato sull'altra (lato carne). I notai sono ormai avvezzi a prendere nota dei dati essenziali preliminari alla compiuta definizione del negozio giuridico in appositi registri che vengono identificati secondo una varia tipologia di denominazioni differenziate su base locale ${ }^{177}$.

Posto che dottrina giuridica e notarile convergono sulla redazione preventiva dell'imbreviatura rispetto all'instrumentum publicum $^{178}$, l'ordine inverso delle scritture dichiarato da Mattarelli può

176 Caso quest'ultimo, della preparazione dell'imbreviatura successivamente al mundum, esaminato in Pecorella, Studi sul notariato a Piacenza, p. 117. Esempi della normativa locale duecentesca tesa a imporre ai notai la tenuta della imbreviature in appositi registri con caratteri ben determinati in ТАмВА, Una corporazione per il potere, p. 183 s. e anche p. 180, ove si richiama la possibilità, per i notai, di redigere soltanto l'instrumentum definitivo da consegnare alla parte quasi esclusivamente in relazione ad atti dal contenuto semplificato.

177 MeYer, Felix et inclitus notarius, p. 143 ss. e p. 150 s. per l'ampia serie di esempi relativi alla denominazione dei registri notarili.

178 Odofredi, In primam Codicis partem, ad l. Contractus, C. de fide instrumentorum (C. 4.21.17), f. $217 \mathrm{vb}, \mathrm{n} .7:$ «[...] ita ista rogatio, scheda et protocolum, nam bene videtis quod tabelliones semper primo scribunt in quaterno suo et ista scriptura vocatur scheda, quasi scissa, vel continens in se id quod agebatur inter contrahentes. Item vocatur protocolum, quia olim a pilo scribebant arrogationes [...], et postquam tabelliones fecerunt schedam, in pulchra charta scribunt instrumentum et tradunt partibus contrahentibus». Il passaggio dalla scrittura preliminare al mundum è chiaro anche in Cino, Lectura super codice, ad l. Contractus, C. de fide instrumentorum, f. 223vb, n. 1 (C. 4.21.17): «[...] contractus qui initus est in scriptis non ante habet efficaciam quam in scheda fuerit positus, quam vulgus appellat rogationem sive rogitum, que est protocollum sive imbreviatura et ex scheda sive protocollo redactus fuerit in mundum». Anche Salatiele, sul punto, non ha dubbi: Salatiele, Ars notarie, I, rub. Quid debeat notarius observare gratia sui of- 
trovare fondamento nelle iniziative messe in campo dalle autorità comunali per tutelare l'interesse delle parti contraenti e l'avvenuta modificazione dei diritti soggettivi attuata mediante la documentazione notarile, soprattutto per negozi di un certo valore economico. Si può cioè interpretare come riferimento alla registrazione affidata ai registri dei Memoriali, demandata ad appositi uffici comunali che nelle realtà di Bologna e Modena funzionano dagli anni Sessanta $\mathrm{e}$ Settanta del Duecento e sono certamente ben noti a Mattarelli.

All'aprile 1265 risale l'istituzione dell'ufficio bolognese dei Memoriali, cui è affidato il compito di conservare i registri nei quali dovevano essere trascritti, gratuitamente e a cura delle parti, nonché a pena di nullità degli atti stessi, gli estremi essenziali tanto dei contratti redatti in città, l'oggetto dei quali superasse le venti lire di bolognini (il valore di una coppia di buoi), quanto degli atti di ultima volontà ${ }^{179}$. Il Comune di Modena sembra essere il primo a imitare l'istituzione bolognese e sin dal marzo 1271 si conservano analoghi registri che si differenziano da quelli bolognesi soprattutto per due elementi: la responsabilità dei notai, sancita fin da subito, nella registrazione dei rispettivi atti e la registrazione dei testamenti, ove risulta obbligatorio riportare anche la menzione dell'erede istituito, secondo una prassi che a Bologna viene introdotta soltanto a partire dal marzo $1290^{180}$.

A Modena - come precisano gli statuti - l'istituzione dei Memoriali assolve primariamente a esigenze di legalità per contrastare la

fitii, p. 17: «Item si contractus est talis qui celebretur in scriptis primum debet facere scedam seu imbreviaturam seu rogationem et postea facere publicum instrumentum» (= II, p. 15).

179 Tамва, Teoria e pratica, p. 46; ID., Una corporazione per il potere, pp. 192 ss., 227 ss. Si veda anche FranchinI, L'istituto dei «Memoriali»; Cesarini Sforza, Sull'ufficio bolognese dei Memoriali; Orlandeldi, I Memoriali bolognesi. In seguito viene sancita la responsabilità della registrazione a carico dei singoli notai.

180 Tамва, Una corporazione per il potere, pp. 247, 252; Sarti, Publicare exemplare - reficere, p. 637 s. La diffusione anche a Modena dell'ufficio dei Memoriali può essere fortemente dovuta all'evoluzione degli equilibri politici interni e all'importazione di modelli amministrativi di matrice bolognese, favoriti dal fatto che nel primo semestre del 1271 è capitano del Popolo a Modena il bolognese Niccolò Baccellieri, passato poi a ricoprire l'ufficio podestarile nel semestre successivo: per riflessioni più ampie e documentate in merito si rinvia a BoNACINI, $I l$ «Registrum Comunis Mutine», p. $19 \mathrm{~s}$. 
redazione e la circolazione di contratti e testamenti falsificati o contraffatti tramite la garanzia offerta dalla genuinità del negozio giuridico fissato negli appositi registri comunali ${ }^{181}$, e tali obiettivi furono perseguiti non senza spunti di originalità rispetto al corrispondente modello bolognese. Nei Memoriali modenesi venivano infatti registrati gli atti rogati non soltanto in città, ma nell'intero distretto, e con una graduazione del tempo concesso per assolvere l'obbligo di registrazione in rapporto alla distanza del luogo di redazione dell'atto dal centro urbano; inoltre, il valore dei beni oggetto delle transazioni registrate oltrepassa le dieci lire modenesi (nonostante l'obbligo statutario scattasse al superamento della soglia più alta di venti lire), corrispondenti al valore di altrettante lire bolognesi, e responsabili delle registrazioni sono individuati da subito gli stessi notai, secondo una prassi che «lascia intravedere una volontà di controllo dell'intera attività notarile per la negoziazione privata molto intensa

181 Statuta civitatis Mutine, 1. I, r. CXXXVIII, De instrumentis ponendis in memorialibus Comunis Mutine a viginti libris Mutine supra, p. 124 ss. Con una soluzione similare a quanto già in vigore a Bologna, i registri dei Memoriali notarili modenesi erano redatti in doppio esemplare e sino ai primi decenni del Trecento depositati ogni semestre l'uno (l'autenticum) in sacristia fratrum predicatorum, l'altro (l'exemplum) in scrineo librorum causarum presso il palazzo comunale. In seguito alla riforma statutaria del 1327 se ne modificarono i luoghi di conservazione: l'autenticum nella sacrestiam sancti Geminiani in scrineo, armario vel loco ad hoc deputato vel deputando (ossia nella sagrestia della chiesa vescovile ricavata in una stanza della torre di S. Geminiano, la Ghirlandina) e l'exemplum nella camera actorum in armario ad hoc deputato: Statuta civitatis Mutine, 1. I, r. CXXXVIII, De instrumentis ponendis in memorialibus Comunis Mutine a viginti libris Mutine supra, p. 127, e r. XLV, Quod turris comunis habeatur pro secrestia comunis, p. 36 s., ove si conferma la decisione, presa forse a seguito delle distruzioni del 1306, di sistemare parte dell'archivio pubblico nella torre del Comune (la Ghirlandina), che perciò avrebbe dovuto svolgere una funzione analoga alla secrestia comunis et camera (degli atti) per custodirvi omnia que ad Comune pertinent et ibi gubernentur, teneantur et ponantur carte et privilegia Comunis que sunt in massaria Comunis. La Camera degli Atti fungeva da archivio generale al quale erano preposti, ogni tre anni, quattro notai con il compito di raccogliere e ordinare la documentazione di interesse pubblico tra cui, principalmente, i libri dei bandi, delle riformagioni, i libri delle entrate e delle spese delle quali erano responsabili i massari del Comune, i registri degli atti giudiziari civili e criminali nonché i libros Memorialium Comunis Mutine: MARChetTI, Inventario dell'Archivio Notarile, p. 2 s.; ToRelli, Studi e ricerche, p. 293 s. Le tipologie di scritture depositate presso la Camera degli Atti sono specificate in Statuta civitatis Mutine, 1. I, r. CXXXIII, De offitio camare actorum et eorum notariis et designatione quam facere tenentur officiales comunis, p. 111 ss. 
e priva affatto di quelle remore che avevano caratterizzato l'istituzione dell'ufficio dei Memoriali in Bologna ${ }^{182}$.

Quanto al riconoscimento dell'autonomo valore giuridico dell'imbreviatura, distinto da quello di mero riscontro probatorio di clausole e contenuti dell'intrumentum laddove questo sia sospettato di falso o quantomeno viziato da errori materiali ${ }^{183}$, i primi passi si devono alla normativa di ambito comunale, interessata a specificare in dettaglio contenuti e forme delle scritture preparatorie, nei decenni attorno alla metà del Duecento, al fine di garantirne un riconoscimento pari a quello dell'instrumentum perfetto ${ }^{184}$.

Su questo piano la dottrina impiega molto tempo ad allinearsi e soltanto con Baldo, nel maturo Trecento, si giunge a ravvisare nell'imbreviatura i caratteri di scrittura "originale" e di "matrice" 185 , purché dotata di alcuni tra i caratteri individuati, per consolidata

182 ТАмвА, Una corporazione per il potere, p. 251 s.: tale prassi sarebbe anche spia evidente del «peso sociale e politico di gran lunga inferiore» acquisito dal ceto notarile modenese rispetto a quello raggiunto negli stessi anni dal corrispondente ceto bolognese. Valutazioni in parte concordi sono espresse in BonAcinI, Scribarum turba Mutine, p. 57 ss.

183 Caso contemplato da Bartolo nel commento alla 1. si librarius, ff. de regulis iuris (D. 50.17.92) (Commentaria, VI, f. 272rb-va, n. 9). Vedi sotto nota 194.

184 Tамва, Una corporazione per il potere, p. 183 s. MeYer, Felix et inclitus notarius, p. 147 ss. per esempi tratti sia dalla normativa statutaria del secondo Duecento sia dalla prassi giudiziaria tesi a riconoscere validità alle registrazioni preliminari del notaio unitamente alla possibilità di ricorrere ad esse nei casi di perdita del mundum o di sospetti sulla sua genuinità.

185 Una scrittura "originale" che tuttavia si può ricostituire nei casi, a quanto pare non infrequenti, in cui vada perduta oppure distrutta anche per effetto di conflitti politici: BaLdi Ubald, In primum, secundum, tertium codicis libros commentaria, 1. et quae a divo, C. de edendo, f. 100va, n. 15 (C. 2.1.8): «[...] quia si notarius perdidit originale idest nativum instrumenti, potest petere a possessore instrumenti, quia edat sibi notario cum die et consule, ut notarius edat alteri, ut ff. eo, l. si quis ex argentariis, § pen. (D. 2.13.6.9), quod tene menti, quia saepe contingit quod notarii, restitutis instrumentis, perdunt protocolla; sicut est saepe in civitatibus, quoniam notarii expelluntur et protocolla comburuntur vel comburitur armarium publicum, tamen apparent quaedam condemnationes inde sumptae, nam illis legitime sumptis tamquam protocollo est standum et habentur pro protocollo, ex quo matrix scriptura non apparet». Sulla medesima linea interpretativa anche Angelo degli Ubaldi nel commento alla l. si quis ex argentariis, ff. de edendo (D. 2.13.6): Angel Ubaldi, In I. atque II. Digesti Veteris partem Commentaria, f. 53vb, n. 1: «Et sic est casus expressus quod licet notarius ediderit instrumentum parti, tamen praesumitur retinuisse penes se primum originale, quod vocamus prothocollum seu imbreviatura, et si in aliquo instrumentum differt a prothocollo, standum 
tradizione della teorica notarile duecentesca, tra le publicationes necessarie a distinguere l'instrumentum redatto dal notaio e pronto per essere consegnato alla parte richiedente ${ }^{186}$.

Il cammino in questa direzione è tuttavia già aperto da Mattarelli, che si mostra propenso a riconoscere autonomo valore probatorio all'imbreviatura parificandola in ciò all'instrumentum publicum, benché con qualche oscillazione che denota ancora incertezze e remore nel dichiararlo in forma convinta e univoca. All'imbreviatura si può attribuire un valore probatorio pari a quello dell'instrumentum sulla base di due ragioni fondamentali: essa habet omnia que debet habere instrumentum [...] ymo habet hoc plus, quia ipsa est originalis cui magis creditur; inoltre, se si presta fede all'atto quod est estractum ex prothocolo, ergo multo magis originali unde trahitur $^{187}$. Ma si deve anche considerare che il protocollo costituisce la fase iniziale dell'atto e quest'ultimo, che ne è il completamento di-

esse prothocollo». Meyer, Felix et inclitus notarius, p. 145: nel corso del Trecento, all'imbreviatura è generalmente riconosciuto il carattere di principalis scriptura.

186 BALDI UbaLDI, In quartum et quintum codicis libros commentaria, C. de fide instrumentorum, Rubrica, f. 57ra, n. 39: «[...] imbreviatura est scriptura rei substantiam continens, cum loco et tempore debitis et testibus et necessariis, ideo sic dicta, quia est quodam modo casus summarius et istam imbreviaturam appellant plurimi matricem scripturam. Secundo quaero, si ista originalis imbreviatura non reperitur, utrum instrumentum parti redditum vitietur, et certum est, quia in civitate Constantinopolitana est de substantia, quod ista matrix scriptura appareat, ut in Auth. de tabellionibis, $\S$ fi. ubi dicit tex. (Nov. 44 = Auth. 45, Coll. IV, tit. 7), quia alias praesumitur instrumentum falsum». Una sintetica ma efficace esposizione delle sei publicationes ritenute necessarie in ТАмВА, Una corporazione per il potere, p. 62 ss. A queste («anno domini, indictio, dies, locus, testes et nomen tabellionis») si aggiunga come settima publicatio, a seconda delle consuetudini locali, il «nomen papae vel imperatoris eo tempore regnantis»: formule tratte dalla rolandiniana Collectio contractuum (1255), riprodotte anche in Atti \& formule di Rolandino, p. 111 (De publicationibus instrumentorum). I caratteri distintivi del publicum instrumentum vengono specificati da Salatiele in apertura del proprio trattato, ma integrati dal signum notarile «quod debet ponere (scil. notarius) in margine extra instrumentum vel in principio instrumenti vel in fine ante suam subscriptionem, et hoc ut, si in posterum dubitaretur fuisset scriptum manu illius tabellionis cuius manu subscriptio innuit esse scriptum, per collationem et comparationem litterarum et signorum possit illud instrumentum confirmari vel eius fidei derogari»: SALATIELE, Ars notarie, I, rub. Quid debeat notarius observare gratia sui offitii, p. 17 s. (= II, p. 15 s.).

187 Vat. Lat. 5773, f. 48va; cfr. MatTarelli, Tractatus super instrumentis, p. 43. 
retto, non ha valore se non è perfezionato in tutte le sue parti ${ }^{188}$. Tra le due scritture permangono quindi differenze non trascurabili che non permettono di collocare l'imbreviatura/protocollo su un piano di piena ed autonoma efficacia legale rispetto all'atto perfezionato in tutte le sue parti, per quanto alla prima si possa comunque attribuire una latitudine e una forza probatoria abbastanza ampia.

L'imbreviatura/protocollo stesa dal notaio deve essere prodotta in giudizio, quando richiesto, per verificarne la piena consonanza con l'instrumentum e quindi confermarne la genuinità, di cui si può invece dubitare se esso manifesta significative divergenze rispetto alla scrittura preparatoria ${ }^{189}$. Scrittura che acquista valore particolare anche quando instrumentum esset nullum ex causa solempnitatis obmisse, in quanto contenente tutti gli elementi sostanziali per la formalizzazione del negozio giuridico ${ }^{190}$ e per la redazione - si può dedurre - di un altro instrumentum completo di tutte le solempnitates in base al pacifico accordo tra le parti ovvero per ordine di un magistrato, nel caso in cui fosse già insorta una lite.

La scrittura preliminare del notaio, pertanto, è in grado di costituire un fondamentale termine di paragone per valutare i contenuti dell'atto perfezionato in tutte le sue parti, ne precostituisce, in un certo senso, l'attendibilità in quanto testo in cui si incontrano e si definiscono in tutti i termini essenziali le volontà delle parti; eccetto i casi in cui, con la complicità del notaio stesso, la parte più forte non riesca a fare inserire nel mundum condizioni ad essa maggior-

188 Vat. Lat. 5773, f. 48va: «[...] ipsa scriptura publica probabit, licet a prima processerit, quamvis prima non probet, quia nundum est quo ad probacionem perfecta nixi fuerit completa per publicacionem [...]»; cfr. MatTarelLI, Tractatus super instrumentis, p. 44.

189 Vat. Lat. 5773, f. 49rb: «[...] ut quandoque oportet ad probacionem vel improbacionem instrumenti, producio prothocoli fieri possit, ut ex eo undique consonante cum instrumento aliquid operetur ad probacionem instrumenti, que quandoque requiritur [...] et ipso disonante manifeste, instrumenti vicium arguatur [...]»; cfr. MatTarelli, Tractatus super instrumentis, p. $52 \mathrm{~s}$.

190 Vat. Lat. 5773, f. 49ra: «Sed hoc fallit, nisi instrumentum esset nullum ex causa solempnitatis obmisse, prothocolum autem esset solempne, ut quia in instrumento non esset omnia ea que ad instrumenti solempnitatem requiritur [...] et idem etiam postest dici quando ea que sunt de substancia alicuius instrumenti continentur in prothocolo <aliter> quam in instrumento [...]»; cfr. MatTarelli, Tractatus super instrumentis, p. 51. 
mente favorevoli tramite l'esplicitazione delle clausole di rinuncia o l'allargamento del raggio di applicazione di quelle eventualmente sottese dalle abbreviazioni inserite nel protocollo. Esempi non eccezionali nella realtà bolognese e ben noti a Odofredo, che ne rammenta la diffusione nel passato in materia di contratti soprattutto per quanto concerne i vantaggi a favore dei creditori ${ }^{191}$.

L'apertura offerta da Mattarelli verso il riconoscimento del valore probatorio delle imbreviature rimane comunque significativa e di portata più avanzata rispetto alle riflessioni di altra dottrina giuridica anche successiva. Cino, infatti, per quanto sembri propenso ad attribuire un valore probatorio autonomo all'imbreviatura, appoggiandosi a Iacopo d'Arena e alla vulgaris opinio da questi richiamata $^{192}$ non abbandona una posizione di prudente diffidenza valutando come ciò sia possibile nisi sit receptum in mundum e quindi, in ultima istanza, essa rimanga condizionata dal confronto con quest'ultimo e dalla maggiore garanzia probatoria da esso offerta. Il protocollum sive scheda è pur validato dalla data cronica e dal signum tabellionis apposto in principio quaterni, ma rimane comunque opportuno che instrumenta habeant suas proprietates, quas non habet imbreviatura, ovvero siano validati da elementi certificanti riconosciuti essenzialmente nella subscriptio del notaio e nel suo signum distintivo ${ }^{193}$.

Un passo successivo è marcato da Bartolo laddove precisa che,

191 OdofRedi, In primam Codicis partem, ad l. contractus, C. de fide instrumentorum (C. 4.21.17), f. 217vb, n. 9: «Et in lex ista municipali huius civitatis est exemplum et in ea ita continetur, ne debitores baratentur per creditores, si contractus excedit summam.xxv. librarum, quod primo debet scribi totus contractus voluntate partium et postea debet eis legi et postea ab eis approbari antequam vocentur testes et postea debet legi presentibus testibus. Nam olim notarii consueverunt ita facere: nam vocabant testes et non scribebant aliquid, sed postmodum cum scribebant, detrahebant et adiungebant ad sensum suum et creditoris».

192 Iacopo D’Arena, Commentarii in universum ius civile, l. Contractus, C. de fide instrumentorum, f. 24ra-b (C. 4.21.17). Anche l'additio posta a integrazione di tale passo, appoggiandosi alla più risalente autorità di Uberto da Bobbio, conferma l'opinione assolutamente prevalente tesa a negare che la scheda sive imbreviatura tabellionis possit per se probare, quia non sit ad probationem, sed ad solam memoriam, giacché solum instrumentum probare debet in quo omnia sigillatim redacta sunt, quia illud fit ad probationem.

193 Cino Da PIstoia, Lectura super Codice, in lib. IV, tit. XXI De fide instrumentorum, f. 224va, n. 14 (C. 4.21.17). 
in caso di errore materiale compiuto dal notaio nel redigere una propria scriptura, allo scopo di verificarne con esattezza il tenore si può ricorrere al protocollo, che assume pertanto la funzione e la definizione di scrittura principalis, dal momento che il notaio difficilmente si limita a redigere $a b$ initio il solo instrumentum ${ }^{194}$. È ad essa che di deve prestare fede in caso di contrasto tra le due scritture perché originalis, in quanto, etimologicamente, predisposta per prima e quindi in grado di assicurare pienamente i contenuti dell'exemplum da essa derivato ${ }^{195}$. E con ciò pare dichiarata senza reticenze la forza autonoma dell'imbreviatura/protocollo, confermata poi da Baldo facendo sempre leva sul connotato di originalità e sulla sua identità di scrittura matrice, ovvero 'madre' di quelle da essa originate e formate in tempi successivi.

Anche la scienza canonistica viene a convergere sulle medesime posizioni. Senza alcuna reticenza Giovanni da Imola, nella sua lettura al secondo libro delle Decretali ${ }^{196}$, dichiara che si può prestare fede al protocollo, dal momento che esso viene letto dinanzi alle parti e ai testimoni, e quale testimonianza contraria riporta proprio l'opinione di Mattarelli filtrata da una Additio di Giovanni d'Andrea

194 Bartolo da Sassoferrato, Commentaria, 1. si librarius, ff. de regulis iuris (D. 50.17.92) (Commentaria, VI, f. 272rb-va, n. 9): «Si vero scripsit (notarius) scripturam propriam, tunc si habet prothocollum probat per ostensionem prothocolli $[. .$.$] nam ex illo verificatum instrumentum extensum et sic vero erratum$ in transcribendo. Sed posses dicere quare dicitur magis principale prothocollum quam publicum instrumentum, nonne potuit esse quod notarius ab ipso initio confecit publicum instrumentum».

195 Ibidem, l. sempronius, ff. de legatis (D. 31.47.un.), IV, ff. 40vb-41 ra, n. 3. «[...] et ideo si producerentur due scripture sive instrumentum publicum et prothocollum notarii, starem potius prothocollo quia ista potius est originalis scriptura [...] et ita hic si constaret unam scripturam primo factam et aliam inde exemplatam, prime starem». Una posizione ribadita, nella sostanza, anche nel commento alla 1. si quis ex argentariis, ff. de edendo (D. 2.13.6), I, ff. 82vb-83ra: «Secundo dico quod intrumentum quod notarius reddit parti non presumitur esse originale, sed originale remanere presumitur apud ipsum notarium, ex quo semper remanet instructus, nisi probet se perdidisse [...]; et ex hoc sequitur quod si est varietas inter instrumentum datum parti et librum notarii, potius est credendum libro notarii».

196 Posto che egli insegna le Decretali dai primi anni del nuovo secolo fino al 1415 in sedi diverse (Ferrara, Siena Padova, Bologna) e poi di nuovo nella città felsinea a partire dal 1422, la Lettura al secondo libro delle Decretali rinvia all'insegnamento tenuto a Bologna nel corso del 1424: PADovani, Dall'alba al crepuscolo del commento, pp. 24 ss., 77 ss. 
allo Speculum ${ }^{197}$. Per quanto il protocollo habeat solennitates, quas habet instrumentum publicum, causa la sua incompletezza rispetto a quest'ultimo e la sua scrittura in forma abbreviata, il maestro modenese tenet contrarium ritenendo che esso non probet. E tale posizione, in cui si rispecchiano le argomentazioni più caute di Mattarelli già sopra richiamate, pare ormai anacronistica al punto da indurre l'autorevole giurista imolese, concludendo la serrata confutazione delle allegazioni assunte a sostegno della tesi opposta, a esortare il lettore a non oscurarne la memoria: tene menti singulariter dictum ipsius Nicolai ${ }^{198}$.

Se queste sono le tappe salienti scandite dalla dottrina giuridica tra Due e Trecento, un percorso nettamente diverso è seguito dalla dottrina notarile di matrice bolognese nel corso del secolo XIII, che non rivolge particolare attenzione alla problematica inerente il valore probatorio dell'imbreviatura/protocollo e che, al pari della dottrina giuridica precedente e contemporanea, non viene fatta oggetto di considerazione da parte di Mattarelli, tutto compreso nell'approfondimento della tematica documentaria alla luce dei soli agganci normativi al ius civile.

Concentrata nella puntuale rassegna formulare tanto gradita ai pratici, ma senza neppure ignorare il più difficoltoso approfondimento della materia secondo la prospettiva dottrinaria, destinato in ogni caso a riscuotere assai minore successo da parte del fiorente notariato cittadino ${ }^{199}$, la Scuola notarile bolognese rivolge scar-

197 Guglielmo Durante, Speculum iudiciale, II, II, De instrumentorum editione, § Videndum, add. In principio, t. I, p. 649: «Sed Nic. contradicens protocollum esse principium instrumenti nondum completi, unde nisi compleatur, pro nihilo est $[\ldots] »$.

198 Ioannis ab Imola, In secundum Decretalium Commentaria, f. 136va, n. 3 $(\operatorname{ad} X .2 .32 .15)$.

199 Non si vuole ripercorrere, in questa sede, la bibliografia dedicata alla produzione dei singoli maestri bolognesi di ars notariae nel corso del secolo XIII e alle differenti impostazioni che distinguono le rispettive opere. Accanto ai fondamentali studi di Orlandelli, Genesi dell'«ars notariae» e ID., Appunti sulla scuola bolognese di notariato, sono importanti le considerazioni di SINISI, Formulari e cultura giuridica notarile, p. 3 ss. e ID., Alle origini del notariato latino, in specie p. 169 ss. per una rassegna delle opere dei principali teorici e pratici della materia e delle scelte compositive che le contraddistinguono. Esaurienti, a tal fine, anche i profili recenti tracciati da Birocchi, Salatiele; Morelli-Murano, Salatiele; Birocchi, Ranieri da Perugia; ID., Rolandino Passeggeri; ID., Zaccaria di Martino. Il più recente 
sa attenzione al problema del rapporto tra instrumenta e scritture preparatorie. Il tema viene appena sfiorato da Ranieri da Perugia il quale, nell'ambito della riflessione sui caratteri generali di pacta et instrumenta, ricordando in forma isolata la redazione di una prima scriptura non contempla alcuna fattispecie per cui essa possa costituire un autonomo mezzo di prova; anzi, essa diviene inaffidabile determinando pure la nullità dell'instrumentum nel caso in cui sia contraddetta da un'altra scrittura o laddove l'instrumentum venga confezionato da un soggetto diverso dal notaio cui si deve - pare di capire - la stesura della prima scriptura ${ }^{200}$. In seguito il solo Pietro d'Anzola, portando a termine il commento avviato da Rolandino alla propria Summa ${ }^{201}$, ammette l'equivalenza ormai invalsa fra rogatio, imbreviatura e protocollo ma non si sofferma a esaminarne il valore probatorio, quanto piuttosto la diversa consuetudine che si è affermata in rapporto alla redazione dell'instrumentum. Un tempo le due scritture erano predisposte in eadem charta sive membrana in modo da poter ricorrere subito all'imbreviatura/protocollo in caso

profilo di Rolandino e della sua opera complessiva, con attenzione soprattutto ai manoscritti che ne conservano testimonianza e alle edizioni a stampa più risalenti in Murano, Rolandino Passaggeri. Pur concentrato su ambiti specifici dell'attività notarile, offre una completa panoramica critica della trattatistica bolognese duecentesca Sarti, Publicare - exemplare - reficere, p. 617 ss.

200 Ranieri da Perugia, Ars Notariae, rub. [VIII] Generalia pactorum et instrumentorum, p. 23: «Circa tenorem sane contractuum sive negotiorum, si fiant de aliqua obligatione, sciendum est, omnia presumi sollempniter acta instrumenta inde confecta. Set si contrarium probetur vel rem aliter esse gestam, quam in instrumento contineatur, vel si tale quid probetur, quo probato, quod in instrumento contineatur, stare non possit vel duo contineat in se repugnantia, vel si sit exemplum, cui fides non hadibetur, nisi autenticum ostendatur, vel si prime scripture per aliam scripturam vel per contradictionem derogetur, vel si sit factum instrumentum ab alio quam a tabellione, cum private scripture non sit habenda fides, et pro nullis habentur talia instrumenta». Appesantite da una fitta trama di allegazioni tratte dai libri legales, le prescrizioni di Zaccaria di Martino in tema di instrumentorum editione ripercorrono fedelmente la traccia del maestro Ranieri, ma in luogo del riferimento alla prima scriptura si legge invece quello alla propria scriptura: ZACCARIA DI Martino, Summa artis notarie, p. 83. Anche per questo scarto del testo di Zaccaria pare che l'attenzione di Ranieri, nel passo sopra indicato, si concentri piuttosto sul rapporto tra scrittura originale (exemplar) e copia che ne viene tratta (exemplum), approfondito in particolare da SARTI, Publicare - exemplare - reficere, p. 648 ss.

201 Sinisi, Nel solco di Rolandino, p. 46; Tamba, Pietro d'Anzola, il "commentatore" di Rolandino, pp. 157 s., 182. Sintetici riferimenti anche nei profili biografici di ID., Pietro d'Anzola (a), p. 1580 e ID., Pietro d'Anzola (b), p. 452. 
di dubitatio sui contenuti dell'instrumentum, mentre hodie de consuetudine non sic fiunt rogationes, che invece sono vergate in quaterno oppure in alia charta [...] de consuetudine hodie in charta de papyro vel in membrana prout magis placet tabellioni, licet instrumentum quod inde trahis postea solum in membrana scribatur ${ }^{202 .}$

\subsection{Le scritture dei mercanti}

Fra le scritture probatorie prodotte extra iudicia, oltre che su quelle redatte da tabelliones investiti di un pubblico ufficio e da quanti, essendone privi, ne sono comandati publica auctoritate come gli argentarii (i banchieri), l'attenzione di Mattarelli si concentra sulle scritture predisposte da un privatus, nel caso di specie identificato con i nummularii altrimenti riconosciuti nostri temporis mercatores $^{203}$. Come produttori di scritture probatorie vergate manu alicuius private persone, i mercanti redigono libri mercatorum che nella puntuale tassonomia disegnata dal giurista vengono ricompresi entro il genere delle scritture realizzate per mandatum accanto a quelle redatte in forma documentorum. Il mercante, infatti, predispone una scrittura in libro suo [...] ex mandato agencium in caso di negozi avvenuti tra estranei oppure ex mandato del medesimo soggetto che effettua un deposito direttamente presso lo stesso mercante $^{204}$.

Non emerge una riflessione sulle tipologie delle scritture mercantili nella loro varietà sia formale che strumentale, ma si pone attenzione ai soli libri rationum, nei quali - in mancanza di ulteriori specificazioni - si possono genericamente individuare i registri dell'azienda mercantile-commerciale destinati a riunire in ordine non necessariamente cronologico tutte le poste aperte relative a debiti e crediti ${ }^{205}$. Diversamente, già nel maturo Trecento appare più evidente la distinzione - precisata da Baldo - fra registri più infor-

202 Rolandini Rodulphini Bononiensis, Summa totius artis notariae, f. 146vb.

203 Vat. Lat. 5773, f. 47vb; cfr. MatTarelli, Tractatus super instrumentis, p. 28.

204 Vat. Lat. 5773, f. 50ra-b; cfr. MatTARelli, Tractatus super instrumentis, p. 66 ss.

205 Tucci, Il documento del mercante, p. 555; Fortunati, Scrittura e prova, p. $54 \mathrm{~s}$. 
mali e privati tenuti dal mercante per propria semplice memoria e i libri mastri ufficiali ove rationes diligentissime perscriptae referuntur e per di più apposte de consensu alterius partis ${ }^{206}$.

Per garantire efficacia probatoria a scritture che esulano dalle modalità legittime di costituzione di una obbligazione, l'analisi di Mattarelli non punta a individuare le regole d'uso delle scritture mercantili in registro in principii di carattere generale, ma le verifica secondo una minuta casistica su base soggettiva, imperniata sulla figura del mercante rispetto al valore che le scritture stesse assumono in relazione a tre categorie ben individuate: quelle che attestano operazioni economiche tra soggetti terzi che il mercante accetta di registrare sulla base di uno specifico mandato consensuale (et haec sunt vera quando mercator scripsit inter extraneos), quelle che registra pro eum (la notazione di un prestito concesso e quindi di un credito aperto) oppure contra eum (la notazione di un deposito ricevuto e quindi di un debito da saldare).

Una distinzione che si applica anche nei confronti di scritture articolate in plures raciones separatas, che danno conto di una serie di operazioni di debito e di credito nei confronti di un medesimo soggetto. In tal caso le poste a favore dello scrivente devono essere sottoposte all'esame del giudice ex qualitate et condicione personarum et ex verisimilitudine facti, mentre quelle contra eum sono probanti se l'autore ne conferma la scrittura factam sua voluntate. Un presupposto che rimane valido anche se la registrazione, indipendente da un insieme di scritture connesse tra loro nel documentare l'intreccio di operazioni con una sola controparte, risulta unicamente contra scribentem, per cui, oltre a riportare la precisa causa obligationis, deve sempre essere corroborata per suam confessionem aut per testes qui fuerunt presentes, eum scripsisse vel per alium sua voluntate. Se tuttavia il mercante nega di esserne l'autore, fino a prova contraria è sufficiente quod in libro suo racionum scriptum sit ovvero che la registrazione compaia assieme a tutte le altre che variamente attestano alia sua debita et credita ${ }^{207}$.

\footnotetext{
206 Baldi Ubaldi, Tractatus solemnis de constituto, p. 630b, n. 6.

207 Vat. Lat. 5773, f. 50ra-b; cfr. MatTARELli, Tractatus super instrumentis, p. $68 \mathrm{~s}$.
} 
La sintetica opinione del giurista è tesa a riconoscere autonomo valore probatorio a questa specifica forma di scrittura in registro, ma per fare ciò è costretta a incastonare entro un orizzonte di carattere pubblico le scritture, come quelle prodotte dai mercanti, di cui in precedenza aveva affermato senza difficoltà la dimensione privata. Gli autorevoli termini di confronto invocati tramite le allegazioni normative di cui egli si avvale corrispondono infatti a tipi documentari di provenienza rigorosamente pubblica come, in primo luogo, il census e i monumenta publica, ovvero i documenti prodotti da un magistratus quali, ad esempio, i gesta municipalia, ai quali la norma giustinianea attribuisce un valore probatorio maggiore di quello dei testimoni ${ }^{208}$. E ancora, altre scritture di carattere indiscutibilmente pubblico richiamate dal giurista sono identificate sin nella manifestazione più alta dello stesso potere imperiale ovvero nel testo della lex, che è di per se stesso evidente e non richiede ulteriori conferme circa forma e contenuto: quod scriptum est in libro Codicis intelligitur lex et voluntate principis scriptum esse, nisi aliud appareat ${ }^{209}$.

Con una traiettoria speculare a quella che, imperniandosi an-

208 Con riferimento alla 1. Census (D. 22.3.10), richiamata anche da CRESCENZI, L'ordine isonomico e il problema della struttura della prova, p. 414, analizzando il valore probatorio del documento, segnatamente di quello pubblico notarile, e della sua considerazione da parte della dottrina di diritto comune. Il processo di translatio degli atti all'interno dei monumenta publica è delineato, di recente, da ID., La rappresentazione dell'evento giuridico, p. 217 ss.

209 Vat. Lat. 5773, f. 50rb; cfr. MatTARELLI, Tractatus super instrumentis, p. 69. A supporto di questo secondo riferimento la più ampia serie di allegazioni utilizzate da Mattarelli fa perno sulla costituzione proemiale De Iustiniano Codice confirmando, § Hunc igitur (Const. Prooem. II.3), laddove si vieta sotto pena di crimen falsi la citazione di qualsiasi altra costituzione non inserita nel Codex, adiectis etiam veterum iuris interpretatorum laboribus, per la soluzione di qualsiasi controversia. Si appoggia anche ad altre norme che richiamano scritture di tipo pubblico cui è riconosciuto valore probatorio: all'Autentica Ad haec (post C. 4.21.19[20]), che equipara la presentazione in giudizio di instrumenta e di scritture tratte da un pubblico archivio; alla l. veteris iuris (C. 5.51.13), che prescrive la stesura, da parte di tutori e curatori, dell'inventario dei beni di pupilli e adulti publice facto secundum morem solitum unitamente all'obbligo di prestare fede soltanto ad esso (et secundum vires eiusdem scripturae patrimonium pupilli vel adulti exigi) e infine alla 1. Ne in arbitris, $\S 1$ (C. 2.55.4.1), ove, all'interno di un dispositivo assai articolato in materia di arbitrato, si prescrive che, accompagnando la convenzione arbitrale dal giuramento solenne e debitamente documentato da parte dei contendenti e dell'arbitro, la decisione di quest'ultimo è da considerarsi direttamente vincolante. La norma sarà oggetto di una successiva riforma tramite una Novella emanata dieci 
che sulla riflessione del giurista modenese, segna l'affermazione del valore probatorio del protocollo notarile, pure ai registri mercantili la dottrina giuridica posteriore giunge a riconoscere senza ripensamenti l'utilizzo in sede giudiziaria. Nel suo noto trattatello dedicato alla materia commerciale e alle scritture dei mercanti, dato a stampa in una forma più circoscritta come commento introduttivo al titolo del Codice De constituta pecunia (C. 4.18) ${ }^{210}$, Baldo, pur operando una distinzione tra codex rationum e quoddam simplex memoriale, giunge a riconoscere ad essi un valore probatorio sostanzialmente equivalente, in particolare nel caso di una registrazione attestante un credito da parte dello scrivente. Per quanto il primo tipo di scrittura si debba ritenere quemadmodum liber authenticus publicus quia mercatores gerunt quasi publice officium, e pertanto ad esso creditur quasi publicae scripturae, che si presume redatta per consenso reciproco delle parti sin dall'origine, pure la seconda tipologia scrittoria si può considerare alla luce della medesima ratio, quia haec scriptura semper loquitur. Et ideo sicut si viva vox loqueretur omni tempore, posset omni tempore assentiri vivae voci ${ }^{211}$. E in analogia con l'allegazione di Mattarelli, ma senza rinviare in modo esplicito al suo trattato, a supporto di tale considerazione il giurista perugino recupera la similitudine con la scriptura legis, che sempre loquatur et semper consentiat ${ }^{212}$.

Sorrette anche da questa legittimante similitudine, le scritture leggibili nei registri dei mercanti, che la dottrina si sforza di isolare dalle altre scritture private per «inglobarle in una categoria atipica

anni più tardi, nel 539: sui contenuti di tali costituzioni si rinvia a SitZIA, Riflessioni in tema di arbitrato.

210 BALDI UBALDI, In quartum et quintum codicis libros commentaria, f. 33rb34ra; definito dallo stesso autore quandam summulam, quae proprie respicit facta mercatorum, essa viene anche diffusa, sia a livello di manoscritti che a stampa, in numerose edizioni, in forma ampliata; si veda ID., Tractatus solemnis de constituto. L'operetta è studiata in particolare da PIERGIOVANNI, Un trattatello sui mercanti di Baldo degli Ubaldi, p. 237 ss., e utilizzata da FortunATI, Scrittura e prova, pp. 30 ss., 56 ss. e passim.

211 BaLdi Ubaldi, Tractatus solemnis de constituto, p. 630b, n. 6. La scrittura vergata nel memoriale si può quindi parificare all'obbligazione assunta dinanzi a testimoni: istud memoriale non plus operatur quam confessio facta ad obligandum coram testibus (ibidem, p. 631a, n. 7).

212 Ibidem, p. 631a, n. 6 in fine. 
e specifica diversa da ogni altra ${ }^{213}$, puntano ad acquisire un autonomo valore probatorio anche sotto il profilo che maggiormente rimane problematico ovvero quando esse documentano un'operazione pro se, ossia a favore del mercante stesso. A fronte di un orientamento incline a negare in questo caso forza probatoria ai libri rationum, ma che si mostra via via disponibile ad attenuare la portata del divieto puntualizzando una serie di eccezioni, deroghe e condizioni particolari, un diverso indirizzo tende piuttosto a considerarla «una prova semipiena da integrarsi con altri elementi, quali il giuramento e l'intervento di un testimone» ${ }^{214}$. Elementi che già Guglielmo Durante ammetteva con fatica per riconoscere come probanti le scritture del banchiere-cambiatore volte a documentare la concessione di un mutuo - e pertanto a lui favorevoli ${ }^{215}$ - e che in seguito Mattarelli ammette per un'altra categoria di registrazioni, ovvero quelle predisposte da tutori e procuratori, seppure nel contesto di una graduazione di diverse fattispecie.

Posto che, in ossequio alla tassativa costituzione di Gallieno del 262 allegata da Mattarelli, in alcun modo si deve prestare fede alle scritture apposte dal mercante unicamente pro $e o^{216}$, nel lessico da lui utilizzato i libri racionum non si identificano con i soli registri mercantili, ma pure con altre tipologie di scritture organizzate per conservare memoria di attività e operazioni a contenuto economico o tali comunque da implicare il riferimento a uscite ed entrate finanziarie connesse a ben precise finalità da parte di chi ex officio derazioni.

213 Fortunati, Scrittura e prova, p. 131 e p. 133 ss. per le successive consi-

214 Ibidem, p. 136.

215 Guglielmo Durante, Speculum iuris, II, II, De instrumentorum editione, p. 655, n. 5: «si nummularius seu campsor nostri temporis, cuius scritpura non est publica, sed privata, ut dixi, dicit in scriptura sua die tali mutuavi centum tali, praesumetur pro scriptura cum adminiculo unius testis vel sacramenti, habita distinctione, cui debeat deferri: quamvis id durum videatur». Una posizione comunque rigettata da Cino, come puntualizza Giovanni D'andrea nell'additio «Videatur» al medesimo passo. Il pensiero di Durante è ricordato da Fortunati, Scrittura e prova, p. 136, che tuttavia lo riferisce a p. 657, n. 7 .

216 Vat. Lat. 5773, f. 50ra; cfr. MatTaRelli, Tractatus super instrumentis, p. 68. L'allegazione richiama C. 4.19.7: «Exemplo perniciosum est, ut ei scripturae credatur, qua unusquisque sibi adnotatione propria debitorem constituit. Unde neque fiscum neque alium quemlibet ex suis subnotationibus debiti probationem praebere posse oportet». 
administrat res alterius. Ne sono prova le registrazioni compilate da quanti si trovano nel ruolo di tutore vel procuratore vel negociorum gestore vel socio, per esercitare il quale essi hanno necessità di annotare expensis eius, cuius sunt negocia, scilicet domini vel pupilli vel societatis riportandole in cartulariis domini vel societatis ${ }^{217}$. Per costituire aliqualem probationem pro scribente, ad esse si può riconoscere valore di prova piena sine alio auxilio o di prova semipiena, quindi da integrarsi tramite giuramento dello scrivente o, in caso di insufficienza di quest'ultimo, tramite aliud adminiculum ${ }^{218}$.

In relazione a questa specifica categoria di scritture, nei primi decenni del Quattrocento l'uso dei libri racionum, traendo ispirazione dal Tractatus de instrumentis di Mattarelli debitamente filtrato da Giovanni D'Andrea, è al centro dell'attenzione di un noto giurista come Giovanni da Imola, docente a Bologna e in altre sedi prestigiose di ius civile e canonicum. Considerata la preliminare suddivisione delle registrazioni apposte in tali libri in tre categorie sulla base del valore probatorio che esse rivestono rispetto al loro autore ${ }^{219}$, pacifica già per Mattarelli, Giovanni si concentra soprattutto sui limiti da riconoscere al principio generale secondo cui de iure communi librum rationum non facere fidem in his quae sunt pro scribente ${ }^{220}$; un principio che il maestro modenese, trattando dei registri mercantili, aveva liquidato seccamente senza ammettere eccezioni: nullo modo stabitur ille scripture ${ }^{221}$. Tra i cinque casi che invece rappresentano altrettante deroghe a tale posizione, Giovanni da Imola introduce la valutazione ex qualitate et quantitate rei de qua in scrip-

217 Vat. Lat. 5773, f. 49va-b; cfr. MatTARELli, Tractatus super instrumentis, p. 61. Al pari di quelle mercantili, esse vengono incluse nella più vasta categoria delle scritture private probatorie, le prime redatte per mandatum e queste ultime per officium civile. 65.

218 Vat. Lat. 5773, f. 50ra; cfr. Mattarelli, Tractatus super instrumentis, p.

219 IoAnNIS AB Imola, In secundum Decretalium Commentaria, tit. De fide instrumentorum, f. 124ra, n. 16: «[...] aut liber rationum continet aliquid pro se aut aliquid contra se aut aliquid tendens ad praeiudicium tertii». Altre citazioni di Mattarelli all'interno del medesimo titolo, sempre mediate tramite Giovanni D’Andrea, sono a f. $123 \mathrm{rb}$, n. 11; f. 123vb, n. 13; f. 124rb, n. 18; f. 124va, n. 18.

220 Ibidem, f. $124 \mathrm{rb}, \mathrm{n} .17$.

221 Vat. Lat. 5773, f. 50ra; cfr. Mattarelli, Tractatus super instrumentis, p. 68. 
tura fit mentio, da rimettersi al giudice soprattutto in casi di modici valori praesertim cum iuramento scribentis ${ }^{222}$, secondo un criterio che Mattarelli aveva già condiviso in relazione alle medesime fattispecie, legate alle spese sostenute dai tutori per sostentare, istruire e alloggiare i pupilli, dotate di verosimiglianza e giustificabili di fronte al giudice da parte dei loro autori, e quindi degne di fede sine alio auxilio ${ }^{223}$.

Con la dottrina del primo Quattrocento e con l'autorevole esempio di Giovanni da Imola e di pochi altri autori, come Paolo di Castro $^{224}$, sono attestati i più tardi rinvii al pensiero di Mattarelli, ormai veicolato assai sporadicamente tramite sue opere circolanti in forma autonoma ma assai più di frequente recuperato in modo puntiforme grazie alle allegazioni incluse in molte opere dei maestri del secolo precedente. La sua memoria si consoliderà piuttosto nella successiva letteratura di ambito bio-bibliografico prodotta in età moderna uscendo dal circuito del diritto vivo per trovare stabile collocazione nell'orizzonte assai più statico della storia giuridica. Una letteratura che si dimostra comunque in grado di fornire indicazioni talora utili sull'articolazione e consistenza della produzione scritta del giurista modenese, ma insieme trasmette imprecisioni notevoli e informazioni del tutto errate che nel successivo capitolo saranno meglio valutate tramite uno specifico esame critico.

${ }^{222}$ Ibidem, f. 124rb, n. 17: il caso prospettato dal giurista riguarda poste relative a spese «in alimentis et doctrina pueri, quem constat profecisse in doctrina, nam talia non fiunt sine expensa [...] unde tam moderata poterit esse quantitas, quia iudex stabit dictae scripturae, praesertim cum iuramento scribentis, ut superius etiam tetigit post Nic. de Mat.».

${ }^{223}$ Vat. Lat. 5773, f. 50ra; cfr. MatTaRelli, Tractatus super instrumentis, p. 65.

224 Vedi cap. III, nota 87. Per altre allegazioni di Mattarelli che affiorano nell'opera di Giovanni da Imola ma sulla base dei riferimenti tratti da Giovanni D’Andrea, Cino, Bartolo e Baldo vedi cap. II, note 138-141. 
CAPITOLO III

\author{
SCIENTIA CLARUS, SED ELOQUIO RUDIS \\ LA MEMORIA DI MATTARELLI \\ NELLA LETTERATURA STORICO-GIURIDICA
}

\begin{abstract}
Sommario: 1. Storia e biografia giuridica fra Quattro e Cinquecento - 2. Da Caccialupi a Panciroli - 3. Un genere nuovo: la bibliografia giuridica - 4. Altri percorsi della bibliografia germanica - 5. Esempi di bibliografie universali e settoriali - 6. Erudizione locale fra Cinque e Settecento - 7. Glorie patrie non soltanto modenesi.
\end{abstract}

\title{
1. Storia e biografia giuridica tra Quattro e Cinquecento
}

Nella produzione letteraria dei decenni e dei secoli successivi all'arco di vita di Niccolò Mattarelli si possono individuare alcuni percorsi importanti lungo i quali emergono tracce della memoria del maestro, del suo insegnamento e della sua speculazione dottrinaria attraverso un fascio molteplice di testimonianze: riferimenti ai dati puramente biografici e a quelli pertinenti la sua attività professionale, incardinata nello Studio modenese e soprattutto in quello padovano; richiami alle sue opzioni interpretative e allegazioni del suo pensiero affioranti nella posteriore dottrina giuridica; accenni, infine, al destino occorso ai suoi scritti e alle opere a lui riconducibili.

Il largo insieme di queste attestazioni può essere esaminato suddividendolo in alcuni filoni ben precisi, anche se non privi di connessioni e rimandi reciproci, dal momento che la pura dottrina e storia giuridica non rimane per nulla isolata, nel contesto della cultura 
dotta di lingua latina che segna a livello europeo gli ultimi secoli del medioevo e quelli dell'età moderna, da altre e diverse forme letterarie e dall'allestimento di repertori bio-bibliografici che ambiscono a pretese di universalità.

Un primo percorso lungo il quale osservare le tracce della memoria postuma del maestro modenese concerne l'ambito della produzione scritta maggiormente orientata alla specifica storia e bibliografia giuridica, ossia alla raccolta di informazioni biografico-letterarie sui più insigni giuristi che hanno marcato le tappe del ius commune e della sua progressiva maturazione su scala europea a partire dai tempi quasi mitici, per alcuni aspetti, del 'capostipite' Irnerio.

Si tratta di un filone letterario che affonda le radici in età umanistica, dalla metà del Quattrocento in avanti, e appare in tempi più precoci rispetto ad altri analoghi, concernenti discipline diverse e inquadrabili nel più vasto genere bibliografico, e si distingue per il fatto di conservare a lungo i caratteri organizzativi e le prassi citazionali tipici dei testi giuridici manoscritti e delle convenzioni affermatesi all'interno della dottrina di origine medievale. In esso, alle specifiche opere dedicate a fornire sillogi bibliografiche se ne affiancano altre organizzate piuttosto su base biografica, ove all'interno dei singoli medaglioni dedicati ai giuristi eminenti si elencano le rispettive opere, in un più fecondo intreccio tra documentazione della scienza giuridica e del prorompente sviluppo che essa ha conosciuto tra medioevo e prima età moderna e rassegna della fitta produzione dottrinaria che da essa è scaturita.

Nessun'altra disciplina «può vantare dei corpora di testi così imponenti come quelli riguardanti le opere del diritto» ${ }^{1}$, che determinano assai precocemente la necessità di strumenti bio-bibliografici per orientarsi nel già vasto mare magnum della letteratura e della storia giuridica e possono essere esplorati per verificare i caratteri assunti dalla memoria dei singoli giuristi e le forme attraverso le quali essa si è cristallizzata nel tempo.

1 SerraI, Storia della bibliografia, III, p. 438 ss. e p. 448 per la citazione. 


\section{Da Caccialupi a Panciroli}

Dopo avere esaminato - nel capitolo precedente - la rilevante testimonianza trecentesca offerta da Guglielmo da Pastrengo, e rammentando la perdita dell'opera storica composta da Baldo, strutturata come un catalogo di giuristi e ancora nota fino verso la metà del secolo XVI quando essa fu sotto gli occhi del Diplovatazio ${ }^{2}$, nel maturo Quattrocento assume un rilievo importante il notissimo trattato di Giovan Battista Caccialupi, modernamente valutato come «una delle prime storie scientifiche della giurisprudenza medievale» ${ }^{3}$.

Nato a San Severino Marche, Giovan Battista Caccialupi (1425 ca.-1496) studia diritto civile e canonico a Perugia e segue una carriera, in prevalenza a Siena, quale giudice delle Riformagioni e lettore nello Studio cittadino, quindi docente di diritto canonico a Roma dal $1484^{4}$. Nel suo noto Tractatus de modo studendi et vita docto-

2 Con riferimento al Tractatus de commemoratione famosissimorum doctorum in utroque iure non ancora identificato: SAVIGNY, Storia del diritto romano, I, p. 487 s.; Besta, Baldo e la storia letteraria del diritto, p. 81 ss.; CoRTese, Il diritto nella storia medievale, II, p. 444; Autographa, I.1., p. 107 (voce curata da G. Murano); Cortese, Baldo degli Ubaldi, p. 151, che lo ritiene probabilmente un elenco delle notizie biografiche «tratte per uso personale dagli autori consultati, in primis da Odofredo». Si ricorda anche l'analoga opera, mai ritrovata e tale da sfuggire a qualsiasi tentativo di datazione, De studio legali adipiscendo, composta da Battista da Sambiagio (1425 ca.-1492), docente a Padova di diritto civile e canonico e con interessi pure in campo feudistico, saccheggiata dall'erudizione cinquecentesca e in seguito a ciò uscita dalla circolazione: Belloni, Professori giuristi a Padova, p. 254; Maffei, Giovan Battista Caccialupi biografo, p. 394; Piccialuti, Battista da Sambiagio, p. 255 Il De studio legali adipiscendo venne consultato e citato pure da Antonio Porcellini, docente di diritto civile presso l'Università di Padova nei primi anni del secolo XVI, per comporre il breve profilo di Mattarelli a margine del nome del giurista modenese registrato nella Matricola del Collegio padovano dei Dottori: ANDRich, Glosse di Antonio Porcellino, p. 10. Un'altra storia della giurisprudenza medievale prodotta dalla cultura umanistica attenta al genere lessicografico e impostata come repertorio di giuristi e delle rispettive opere si deve al milanese Catelliano Cotta (...1512-1553) con il suo De iurisperitis (stampato in TUI I, f. 137va139va; cfr. Di Renzo Villata, Cotta Catelliano, p. 601).

3 Cortese, Alle origini della scuola di Bologna, p. 26.

4 Si veda l'accurata voce di D'Amelı, Caccialupi Giovan Battista, particolarmente attenta alla tradizione manoscritta e a stampa delle sue opere; più sintetica ed essenziale quella di QuAglioni, Caccialupi Giovanni Battista. Alla lunga carriera da lui svolta nella città senese come giudice e docente di diritto civile è dedicato il più recente e vasto studio di NARDI, Giovanni Battista Caccialupi a Siena. Una aggiornata sintesi del percorso biografico e scientifico del giurista marchigiano, con 
rum, pubblicato a livello accademico nell'aprile del 1467 e stampato per la prima volta a Venezia nel $1472^{5}$, a una parte introduttiva dedicata alla caratteristiche morali e spirituali che devono possedere quanti si accingono allo studio del diritto, segue la puntuale e ordinata rassegna dei giuristi, soprattutto civilisti, che hanno segnato la tradizione delle Scuole della Glossa e del Commento a partire da Irnerio $^{6}$. Nella ordinata sequenza di oltre 150 brevi profili bio-bibliografici dei Maestri del diritto civile, canonico e feudale che costituisce «l'aspetto nettamente innovativo del trattato del giureconsulto settempedano» ${ }^{7}$, tra il richiamo a Oldrado e quello al bologne-

attenzione soprattutto ai manoscritti autografi sinora rintracciati, in Autographa, I.1., pp. 250-254 (voce curata da G. Murano).

5 La sola sezione bibliografica è nuovamente pubblicata da C.G. Hoffmann nell'edizione del 1721 di Guidi Panziroli, De claris legum interpretibus. Sui caratteri e più ampi contenuti della specifica opera si rinvia a SERRAI, Storia della bibliografia, III, p. 438 s.; DionisotTI, Filologia umanistica e testi giuridici, p. 192 ss.; MafFEI, Giovan Battista Caccialupi biografo, ove in part. a p. 396 s. si documentano le inesattezze riscontrabili nell'editio princeps rispetto alle due versioni manoscritte prese in esame; Frigerio, Umanesimo del diritto, e successive brevi note in ID., Martino da Fano e $i$ De modo studendi, p. 63 s. Si veda anche la scheda sull'opera di Caccialupi in Cоснетті, Repertori bibliografici del Cinquecento, p. 21. Alcune tematiche particolari riferibili alla produzione dottrinaria del giurista marchigiano sono state approfondite in anni recenti da Di Noto Marrella, La nobilitazione del professore, p. 75 s.; Ascheri, La normativa di diritto comune per lo straniero; ID., G.B. Caccialupi (1420 ca.-1496) fautore dei Monti di Pietà, p. 646 ss. In merito a quest'ultimo profilo, le riflessioni di altri giuristi anteriori a quelle di Caccialupi, alle prese a Perugia con il funzionamento della nuova istituzione creditizia, sono analizzate in Muzzarelui, Candelabrum lucem ferens. Accanto all'opera di Caccialupi, una delle più precoci guide orientate all'istruzione e all'educazione dei giovani giuristi è quella notoriamente compilata da Matteo Gribaldi Mofa, De methodo ac ratione studendi libri tres. Sul giurista chierese e la sua opera, che tuttavia non si sviluppa tramite una rassegna di biografie di giuristi, si rinvia al documentato profilo di Quaglioni, Gribaldi Moffa Matteo.

6 Notizie su Irnerio prese direttamente da Odofredo e con errori, circa un presunto insegnamento originario di Irnerio a Roma, introdotti in una edizione tardo quattrocentesca del trattato di Caccialupi: CORTESE, Alle origini della scuola di Bologna, p. 26 s.

7 MAFFeI, Giovan Battista Caccialupi biografo, p. 398, e già nella sostanza ID., Di un inedito «De modo in iure studendi», p. 317: nell'opera ancora inedita composta in età giovanile da Diomede Mariconda (1455 ca.-1511 ca.) intorno al 147580 , che si viene a collocare nel solco autorevolmente rappresentato da Caccialupi, la sezione più originale non è costituita dalle vitae doctorum, ma dalla storia della Studio partenopeo, benché composta in chiave esclusivamente encomiastica in funzione del dedicatario, Enrico Lugardo, governatore della medesima università. 
se Giovanni Calderini un succinto ricordo è riservato pure al doctor mutinensis Nicolaus de Mattarellis, contemporaneo di Giovanni D'Andrea e autore di pulchras lecturas dedicate a Digesto e Codice, purtroppo di ormai rara disponibilità ${ }^{8}$.

Diversamente dall'analoga opera del padovano Giovanni Iacopo Cani (1425 ca.-1494), che vive negli stessi anni insegnando diritto civile e canonico nello studio della propria città ma che nel suo $D e$ modo studendi in utroque iure si concentra unicamente sugli aspetti morali e materiali degli studi giuridici e sulle qualità severamente richieste a quanti vi si accostano ${ }^{9}$, Caccialupi allarga lo sguardo

Sull'autore, professore nello Studio napoletano, giudice e avvocato, si veda anche il recente profilo bio-bibliografico di MAFFEI, Mariconda Diomede.

8 Caccialupi, Tractatus de modo studendi, \& vita doctorum, f. 3va: «Nicolaus de Mattarellis doctor mutinensis fuit contemporaneus Io. And. ut colligitur in addi. Spe. in ti. de confes. in $\S$ sequit. Edidit pulchras lecturas super ff. et C. illarum copia rata (sic) est». Considerato il significato, difficilmente accettabile in tale contesto, di rata, sia come forma verbale che come aggettivo, pare preferibile considerarlo un errore di stampa per rara. Ciò trova conferma in una edizione settecentesca del medesimo trattato: J.B. de Gazalupi, Succincta historia interpretum et glossatorum iuris, in Guidi Panziroli, De claris legum interpretibus (ed. Lipsiae 1721), pp. 501-511, a p. 505 s.: «Nicolaus de Materellis, doctor mutinensis, fuit contemporaneus Joannis Andreae, ut colligitur in additionibus ad spec. in tit. de confessis, $\S$ sequitur. Edidit pulchras lecturas super ff. et $\mathrm{C}$. illarum copia rara».

9 Johannes Jacobus Canis, De modo studendi in utroque iure, dato alle stampe nove anni dopo l'analoga opera di Caccialupi e di cui si ricorda la riedizione ottocentesca curata da Pescatore (ID., De modo studendi in iure libellus). Si tratta di un'opera breve che si può ritenere «il primo manuale giuridico scritto, bene o male, nella nuova lingua, nel latino degli umanisti, non più in quello dei giuristi», dedicata alle norme di comportamento suggerite agli scolari, alle modalità più opportune per accostarsi allo studio di leges et canones e ai principali contenuti dello studio medesimo: si veda in merito DionisotTi, Filologia umanistica e testi giuridici, p. 194, da cui la citazione; MAFFEI, Di un inedito «De modo in iure studendi», p. 304 s., con ulteriore bibliografia anteriore; MAFFeI, Giovan Battista Caccialupi biografo, p. 394. Sul giurista padovano si vedano pure i più ampi profili bio-bibliografici di BELLONI, Professori giuristi a Padova, p. 259 ss.; Ricciardi, Cani Giovanni Iacopo; BirocchIMattone, Can (Cane, a Canibus, de Canibus) Giovanni Giacomo. Al Cani si deve anche un trattatello De tabellionibus, edito già nel 1482 e poi riprodotto all'interno di pregevoli cinquecentine (nella Summa artis notariae di Rolandino a partire dall'ed. Venetiis 1546) ed è l'unico autore, dopo Baldo, a occuparsi in modo specifico della materia del tabellionato. A lui si deve pure un trattato De executoribus ultimarum voluntatum, nella cui Praefatio sono ricordati gli scritti sulla stessa materia composti da noti giuristi del secolo XIII, in seguito ripresi da Giovanni d'Andrea nella sue Additiones allo Speculum iudiciale di Guglielmo Durante, tra i quali Niccolò Mattarelli (f. 185vb: «Eodemque modo Ia. de Are. doctor illustris, com- 
a una nutrita rassegna di civilisti e canonisti che hanno marcato le tappe dello sviluppo della scienza giuridica nell'arco di oltre tre secoli a partire dall'ormai consolidata figura di quel Guernerius seu Hirnerius che per primo incepit glosare textus ${ }^{10}$. In tale organica sequenza, priva di cesure e di valutazioni sui differenti orientamenti metodologici, trova posto anche il richiamo a Mattarelli segnato da brevi e distintive notazioni: l'origine modenese, che appare correttamente individuata, la contemporaneità rispetto a Giovanni d'Andrea, sulla base di una attestazione fornita da quest'ultimo ${ }^{11}$, e la scarsa circolazione dei suoi scritti, già percepibile in età umanistica, frutto di lecturae scaturite assai verosimilmente dalla pratica didattica. Dati precisi e circostanziati che risultano ormai indipendenti dall'ambiente padovano ove per anni Mattarelli ha svolto la propria attività professionale e sono ora in grado di innestarsi nella tradizione della cultura e della storia giuridica entro un orizzonte decisamente più largo.

Nella varia e per certi aspetti imprevedibile circolazione di testi giuridici frutto della ricchissima produzione degli ultimi secoli del Medioevo si può osservare un accostamento diretto tra Mattarelli e il più tardo Caccialupi - per quanto casuale e frutto del vario inserimento entro una silloge miscellanea - in un codice conservato presso la Biblioteca Angelica di Roma scritto nel corso dell'ultimo quarto del secolo XV da Bentivoglio de Bentivoleis, negli anni in cui egli svolge diversi incarichi pubblici tra Stato della Chiesa e Mezzo-

pendium fideicommissarium appellatum, quadrifariam divisum, dereliquit. Tractatumque de his fecere Ubertus de Bobio, Rof. et Odo., Iac. Balduinus, Nicolausque Mattarelli mutinensis, quos Spec. et Ioan. And. in addi. consecuti sunt»). Nelle Additiones allo Speculum emergono riferimenti al pensiero di Mattarelli relativamente alla materia successoria nello sviluppo del titolo Testamenta qualiter impugnentur e del titolo De ultimarum voluntatum executoribus: ad Spec. 2, 2, De instrumentorum editione, $12 \S$ Compendiose (Testamenta qualiter impugnentur), add. penulti$m o$ (vol. 1, p. 681); ad Spec. 2, 2, De instrumentorum editione, $13 \S$ Nunc vero (De ultimarum voluntatum executoribus), add. Nulli (vol. 1, p. 715).

10 Caccialupi, Tractatus de modo studendi, \& vita doctorum, f. 3ra.

11 Guglielmo Durante, Speculum iudiciale, 2, 2, De confessionibus, 2 Sequitur videre (Quis sit confessionis effectus), add. $\S$ II (t. 1, p. 605): «Hoc posuit, quia cum ipso domino Nico. Paduae pro quodam consilio, quod dare debebamus, satis habui de praedictis conferre $[\ldots] »$. 
giorno campano ${ }^{12}$. In oltre 500 carte in formato di 'vacchetta' (mm 295 x 110) è ricopiata una vasta serie di casus, quaestiones disputate e non, repetitiones, tractatus, consilia, commenti brevi attribuiti a un ampio ventaglio di giuristi noti e meno noti: Baldo, Angelo degli Ubaldi, Angelo da Amelia, Bartolo, Pietro de Suntino, Mansueto da Perugia, Oldrado da Ponte, Guglielmo da Perugia, Dino del Mugello, Giovanni di Dio, Giovanni Calderini, Luca da Penne, magister Pontius, Jacopo d'Arena, Gabriele Fabricii da Perugia, Signorolo da Milano, Pietro d'Ancarano, Giovanni de Zochis da Ferrara ${ }^{13}$. Accanto a queste opere si rileva la presenza del trattatello di Caccialupi $D e$ suspectis et fugitivis debitoribus, composto dal giurista marchigiano prima del $1470^{14}$, e di un consiglio attribuito domini Nicolai de Mactarellis in tema di successione ereditaria che presenta allegazio-

12 Roma, Biblioteca Angelica 275 (già C. 6.1). Per la descrizione del manoscritto si rinvia a NARDUcCI, Catalogus codicum manuscriptorum praeter Graecos et Orientales in Bibliotheca Angelica, pp. 148-151, e al più recente Di Cesare, Catalogo dei manoscritti in scrittura latina, pp. 52-54. Il Bentivogli è vicario del podestà di Urbino, quindi iudex presidiatus Farfensis (in Santa Vittoria in Matenano), poi Capitano della città di Nola, Governatore di una terra non meglio specificata in Abruzzo, Segretario del principe Antonello di Salerno e infine, nel 1484, uditore a Roma per conto dello stesso principe presso papa Innocenzo VIII: le precise indicazioni relative agli incarichi da lui svolti, affiancate al ricordo della nascita dei figli, sono registrate nella carta finale del manoscritto.

13 L'opera, attribuita a un non meglio specificato Ponzio, ha come titolo: Tractatus magistri Pontii per quem scitur ubi materia cuiuscumque tituli Decretalium tractatus in Decretis, et primo de titulis primi libri et postea successive (ff. $208 \mathrm{v}-210 \mathrm{r}$ ); l'autore è presuntivamente identificato da Narducci (vedi nota precedente) con Dominicus de Ponte, lettore a Padova del Decreto. Oltre ai numerosi testi giuridici, il manoscritto contiene in apertura alcuni passi tratti dal De civitate Dei di S. Agostino e dalle Epistole ai Romani di S. Paolo, e successivamente anche brevi estratti dal commento di S. Giovanni Crisostomo al vangelo di Matteo (f. 94v) e da un'opera non meglio specificata di Cataldino Boncompagni de Visso (f. 95r). Ai ff. 504r-506r sono riportati anche estratti da autori classici (ex veteribus poetis) ossia Cicerone, Pomponio e Strabone.

14 Come si rileva dall'annotazione apposta nello stesso codice a f. 98r (f. 103r moderna): Finit opus de suspectis et fugitivis, editum per famosissimum virum iurisconsultum dominum Ioannem de Caccialupis de Sancto Severino, maiorem iuris civilis cathedram Senis regentem. Scriptum per me Bentivolum de Bentivoleis, minimum v(irum) i(iuriconsultum) d(ominum), quod perusie anno 1470 de mense septembri scribere incepi, et in Sancta Victoria anno Domini 1475, die XVIIII Octobris explevi, tempore quo eram iudex presidiatus farfensis. Laus Deo. Sulla base del manoscritto dell'Angelica tale caposaldo cronologico è rilevato anche da D’Amelo, Caccialupi Giovan Battista, pp. 791 e 794. 
$\mathrm{ni}$ - per quanto è dato comprendere dall'agile e compendiata scrittura corsiva - unicamente civilistiche ${ }^{15}$.

A una quarantina di anni di distanza dalla testimonianza di Caccialupi si situa la più matura - e ugualmente notissima - opera di Tommaso Diplovatazio redatta entro il 1511 e perfezionata nell'arco di un paio di decenni allo scopo di tramandare una complessa raccolta di biografie di giuristi medievali quale parte di un più vasto Tractatus de praestantia doctorum sviluppato in $12 \mathrm{libri}^{16}$. Si tratta di una ricca cronaca letteraria dei maestri dei secoli precedenti compilata alla luce della citazione delle rispettive opere e della precisa memoria che di essi si conservava nella letteratura giuridica prodotta sino al tardo secolo XV.

In tale rassegna viene incluso pure Niccolò Mattarelli, del quale il Diplovatazio, grazie alla conoscenza di una vasta dottrina giuridica unita alla frequentazione giovanile dell'ambiente padovano, ove egli studia per un biennio tra 1486 e 1488 seguendo i corsi pure di Giason del Maino, fornisce un ritratto più corposo rispetto $\mathrm{a}$ quello delineato dal maestro marchigiano e, soprattutto, ancorato a solide testimonianze. Di Mattarelli, in particolare, oltre alla corretta origine modenese e alla produzione di «solemnes et accuratas [...] lecturas» ormai di rada circolazione, nel solco del succinto medaglione già tracciato da Caccialupi, egli riunisce una serie di notizie assunte da una breve schiera di giuristi sia contemporanei allo stesso Mattarelli, sia di qualche generazione successiva e

15 Biblioteca Angelica 275, ff. 207r-208v: Consilium domini Nicolai de Mactarellis. Inc.: Punctus quaestionis talis est. Abbas monasterii ex tales [...]; expl.: causa allegationis [...] salvo semper conscilio melius dicentis. Nico de Matta.

16 Per un primissimo orientamento sull'opera e sul suo autore, studente di diritto a Salerno, Napoli, Padova, Perugia e quindi addottoratosi a Ferrara nel 1490, si rinvia alle voci bio-bibliografiche di Mazzacane, Diplovatazio Tommaso, e di Ascheri, Diplovatazio (Diplovataccio) Tommaso. Il De claris iurisconsultis dispone di due distinte edizioni a stampa: una prima parte curata da Gustav Pescatore nel 1890 (Pescatore, Thomae Diplovatatii opus de praestantia doctorum) che si conclude con la biografia di Accursio, e una seconda, concernente la parte successiva del testo del Diplovatazio, avviata da Hermann Kantorowicz e Fritz Schulz con una introduzione generale all'opera e una biografia dell'autore (KANTOROWICZ-SchULZ, De claris iuris consultis, con le integrazioni e correzioni di Ascheri, Saggi sul Diplovatazio, pp. 9 ss., 110 ss.) poi completata da Giuseppe Rabotti (DiplovatatiI, Liber de claris iuris consultis). 
in buona parte legati al comune studio o insegnamento presso lo Studio padovano ${ }^{17}$.

Da Cino egli trae informazioni circa la sua patria di origine e la prolungata attività di insegnamento ${ }^{18}$ rammentando pure la riflessione di Mattarelli circa i rapporti fra consuetudo e lex allegata dal maestro pistoiese ${ }^{19}$. Ricorda inoltre con precisione i richiami al giuri-

17 Diplovatatil, Liber de claris iuris consultis, p. 255: «Nicolaus de Matherellis, patria Mutinensis, vir magni ingenii maiorisque doctrine et iuris consultus celeberrimus per hoc tempus Padue multo in pretio legendo e scribendo floruit. Qui et ipse in digesta et codicem sollemnes et accuratas conscripsit lecturas, de quibus rara habetur copia. Fecit etiam et disputavit plures questiones, quas Albericus de Rosato dicit esse in libro magno questionum disputatarum per ipsum in 2. parte statutorum in questione 50. Et vide in questione 32. in dicta 2. parte, et Iovanem Andree in regula quod quis, et in regula nullus in sexto. Ipsum allegat Cinus in 1 . I. in fine C. que sit longa consuetudo, et Iovanes Andree in tit. de notoriis criminibus § iam de notorio v. penultimo, et in tit. de locato $\S$ iam dicendum in additione magna, et in tit. de penis in princip., et tit. de inquisitione $\S$ viso igitur v. 'porro' cetera. Facit mentionem de Francisco de Matherellis fratre dicti Nicolai in questione, incipt: 'Iudex habet merum' et dicit, quod Franciscus refert Nicolaum eius fratrem tenuisse. Floruit tempore supra dictorum et fuit contemporaneus Iovanis Andree, ut per Iovanem Andree in additione Speculatoris in tit. de confessione $\S$ I. in I. additione magna, ubi dicit se satis habuisse conferre cum dicto Nicolao Padue pro quodam consilio, quod dare debebant insimul. Et Cinus dicit in 1. I. in 10. columna C. de sententiis que pro eo quod interest cetera, quod prefatus Nicolaus fuit doctor Mutinensis et longo tempore rexit. Et Bartolus in 1 . filio preterito sub numero 35 . ff. de iniusto et irrito facto testamento, inquit Nicolaum de Matherellis dominum suum antequam fuissent facte alique glosse posite in dicta lege disputavit decisionem in eis contentam. Et de ipso Nicolao vide librum de scriptoribus ecclesiasticis carta $128 »$.

18 Cino da PIsTOIA, Lectura super Codice, in lib. VII, tit. XLVII De sententiis, f. 460vb: «Nicolaus vero Matharellus mutinensis doctor, qui longo tempore rexit in studio paduano [...]». L'origine modenese di Mattarelli è ribadita ibidem, in lib. VII, tit. LIX De confessis, f. 465vb: «Secundum Nico. Mat. de Mutina doct. studii Paduani [...]». Si rammenta che tramite l'opera di Cino una debole conoscenza di Mattarelli filtra anche in ambiente orleanese, ove se ne trova menzione nella lettura al VI libro del Codice composta nel tardo secolo XIV da Jean Nicot e trádita, in forma parziale, nel codice vaticano Borghesiano 92: DuYnsteE, An unknown fourteenth century lecture of the Orléans school, in part. p. 371. L'allegazione di Mattarelli, inserita nell'ambito del commento al titolo de collationibus, l. ut liberis (C. 6.20.17), compare in riferimento al medesimo passo del Codice: CINO DA PISTOIA, Lectura super Codice, in lib. VI, tit. XX De collationibus, f. 361rb, n. 4. Su Jean Nicot (1320ca-1383), maestro di diritto civile a Orléans e quindi vescovo della stessa diocesi dal 1371, si vedano anche CORTESE, Controversie medievali sul dominio utile, p. 627 ss., e il più complessivo profilo biografico tracciato da DuynsteE, $\mathrm{Ni}$ cot Jean.

19 Cino Da PISTOIA, Lectura super Codice, in lib. VIII, tit. LII Quae sit longa consuetudo, f. 520rb. 
sta modenese contenuti nelle opere di Giovanni d'Andrea, collega di Mattarelli a Padova tra 1306 e 1309: nella lettura al $\operatorname{Sextus}^{20}$ e, più numerosi, nelle additiones allo Speculum iudiciale composto da Guglielmo Durante in due redazioni successive tra gli anni Settanta e Ottanta del Duecento ${ }^{21}$. In questo caso viene registrata una sequenza numerosa di allegazioni del pensiero di Mattarelli, unitamente a quaestiones da lui pubblicamente dibattute e a un consilium formulato a Padova assieme allo stesso Giovanni, ed è ribadito l'equivoco circa il legame famigliare con il figlio Francesco, parimenti doctor iuris ma erroneamente indicato come fratello di Niccolò ${ }^{22}$.

Dall'opus statutorum di Alberico, formatosi sempre a Padova alla scuola di Oldrado da Lodi e di Riccardo Malombra, il Diplovatazio trae i riferimenti a due quaestiones importanti nella misura in cui sono utili a precisare il largo impegno di Mattarelli nella dimensione pratica e applicata del diritto tramite la disputa di quaestiones e la loro circolazione all'interno del liber magnus questionum disputatarum giunto nelle mani del maestro lombardo e da lui ampiamente utilizzato ${ }^{23}$.

Dalla vastissima opera di Bartolo che Diplovatazio ben conosce e si impegna a pubblicare anche in seguito al soggiorno perugino

20 GIOvanni D’ANDREA, In titulum de Regulis iuris Novella Commentaria: regula LXII, Nullus ex consilio (f. 54ra); regula XXIV, Quod quis mandatum (f. $81 \mathrm{rb})$.

21 Contese, Il diritto nella storia medievale, II, p. 379.

22 Guglelmo Durante, Speculum iudiciale, III, I, De notoriis criminibus, 8 $\S$ Iam de notorio (Notorium quid sit et quot modis dividatur), add. Ut nostrum (t. 2, p. 52); IV, III, De locato, $3 \S$ Iam dicendum restat (De instrumentorum forma super locatione rerum se moventium), add. Prima (t. 2, p. 262); IV, IV, De poenis, add. Praelibatum (t. 2, p. 516); III, I, De inquisitione, 3 § Viso igitur (In inquisitione qualiter sit procedendum), add. Terminum prefigat (t. 2, p. 36, ove si ricorda erroneamente Niccolò come fratello, anziché padre, di Francesco); II, II, De confessionibus, 2 S Sequitur videre (Quis sit confessionis effectus), add. $\S I I$ (t. 1, p. 605).

23 Alberico da Rosciate, Commentarium de Statutis, II, 32. Si veda Romano, Le quaestiones disputatae, n. 48, p. 101, richiamata pure alla n. 86, p. 123. Alberico conclude la citazione ricordando che questa sarebbe la quaestio n. 302 all'interno del liber magnus quaestionum disputatarum. Per altri riferimenti alle quaestiones attribuite a Mattarelli e utilizzate da Alberico, in riferimento anche al loro inserimento all'interno di un liber magnus a disposizione del maestro lombardo dedicato alla raccolta di tale materiale dottrinario, si veda sopra, cap. II. 
avvenuto tra 1489 e $1490^{24}$, e in specie dalla Lettura all'Inforziato, egli coglie un ulteriore aspetto connesso alla figura del maestro modenese laddove, confermando quanto già filtrato dalla testimonianza di Alberico, ne evidenzia la propensione alla disputatio, che si rileva come un tratto caratterizzante la sua attività didattica e scientifica con particolare attenzione, nel caso esaminato, all'applicazione di una norma in materia successoria. Fraintende invece i termini del rapporto accademico tra Mattarelli e Bartolo, il quale, in realtà, non considera il primo quale dominum suum - come invece sostenuto dal Diplovatazio - ma ne rammenta soltanto l'opinione, circa la validità del testamento dell'usuraio, raffrontandola a quella di Giovanni d'Andrea ${ }^{25}$.

È infine rilevante che Diplovatazio, concludendo il medaglione dedicato a Mattarelli, esorti a vedere pure il librum de scriptoribus ecclesiasticis, opera di erudizione bibliografica pubblicata soltanto un decennio prima da Iohannes von Heidenberg, meglio noto come Trithemius (Trittenheim, 1462-Würzburg, 1516), abate dei cenobi benedettini di Sponheim e Würzburg che nel 1494 stampa a Basilea un repertorio basato sulla raccolta di notizie relative a circa mille autori dell'era cristiana fino ai tempi suoi ${ }^{26}$. Nella scelta di un testo

24 Sull'edizione dell'opera bartoliana curata da Diplovatazio nel corso degli anni Venti del Cinquecento: Ascheri, Saggi sul Diplovatazio, p. 28 ss.; Cortese, Intorno all'edizione di Bartolo. Più di recente, sul rapporto con le opere bartoliane e la loro materiale disponibilità torna Colli, La biblioteca di Bartolo, p. 73 ss.

25 BARTOLo, In primam Infortiati partem, ad l. filio preterito, ff. de iniusto et irrito facto testamento (D. 28.3.17), f. 102v: «Quando glossavit [scil. Ioannis Andreae] Clementinam in c. I, de sepolturis, dixit quod testamentum reconvalescit quoad sepolturam et ita intellexit dictum suum in d. c. quamquam de usuris, 1. 6, dominus Nicolaus de Mattarellis antequam fierent iste glosse disputavit hanc questionem et determinavit quod testamentum reconvalescat et ut tollantur omnia propria, dixit sic». Sulla scorta di VeDRIANI, Dottori modonesi, p. 41, anche Muratori considera Bartolo discepolo di Mattarelli: AIMAe, III, Dissertatio quadragesimaquarta, col. 908.

26 Trithemius, Liber de scriptoribus ecclesiasticis, f. 85r-v (ed. Coloniae 1531, f. 108v), ove si può leggere il profilo di Nicolaus Mutinensis sul quale si ritornerà in questo stesso capitolo, nota 84. Prima grande e organizzata bibliografia dell'età moderna, composta tra 1487 e 1492 e contenente 963 profili biografici posti in sequenza cronologica, arricchiti da oltre 6.000 citazioni bibliografiche riferite con notevole scrupolosità ed esattezza: CocHEтTI, Repertori bibliografici del Cinquecento, p. $23 \mathrm{~s}$. Accanto a opere di carattere teologico e religioso, egli ne compone anche altre di impianto biografico dedicate a gruppi specifici di autori, in specie eccle- 
anche di questo tipo, che allarga e diversifica il repertorio della sola dottrina giuridica dominata da un esperto della materia, emerge il gusto maggiormente letterario e umanistico del Diplovatazio, capace di affiancare ad alcuni grandi maestri della stagione del Commento una delle prime testimonianze del filone enciclopedico-biografico che la giovane cultura umanistica di impronta erudita è stata in grado di produrre dando un rilievo anche letterario, come autori di testi scritti nell'orizzonte della cultura latina di respiro europeo, ai giuristi di età intermedia.

Nonostante l'errato riferimento a un passo bartoliano concernente un'opinione di Mattarelli, il contributo del Diplovatazio accresce in misura significativa, dal punto di vista qualitativo, il breve profilo risalente al Caccialupi fissando nella memoria del primo Cinquecento un ritratto del giurista modenese delineato attraverso precisi, ancorché limitati, richiami estratti da una rassegna di opere dottrinarie tutte risalenti all'epoca più prossima allo stesso Mattarelli. Richiami che sono in grado di fornire una prima misura del travaso nella letteratura giuridica, soprattutto in quella cronologicamente più vicina alla sua scomparsa, del ricordo che di lui permaneva quale docente di diritto civile e autore di opinioni e riflessioni divenute patrimonio circolante nella dottrina del secolo XIV.

A pochi decenni di distanza il ricordo di Mattarelli è rilanciato da Marco Mantova Benavides (1489-1582), prestigioso e longevo docente di diritto civile e canonico presso l'Ateneo padovano, ove ricopre varie cattedre a partire dal $1515^{27}$. Frutto della spiccata sensibilità umanistica e dell'attenzione all'educazione giuridica, considerata quale baricentro della formazione culturale da impartire ai gio-

siastici, come il De ortu et progressu ac viris illustribus ordinis gloriosissimae Dei genitricis sempre virginis Mariae de Monte Carmelo (1492), il De viris illustribus ordinis S. Benedicti (1493) e il Catalogus illustrium virorum Germaniae (1495), prima storia della letteratura tedesca data alle stampe: Widman, Nachwort, pp. II, VI. Un'analisi approfondita dell'opera di Trithemius, al secolo Johann Heidenberg, considerato il primo autore moderno nel settore delle bibliografie stampate poi perfezionato da Conrad Gesner (1516-1565), in SerraI, Storia della bibliografia, I, pp. 31 ss., 257 ss.

${ }_{27}$ Per un complessivo quadro biografico-professionale si veda ZendRI, Mantova Benavides Marco Antonio, che per gran parte compendia la precedente voce biografica di Tomasi-Zendri, Mantova Benavides Marco. 
vani, è la Epitoma virorum illustrium, data alle stampe nella sua città natale e concepita come rassegna dei maggiori giuristi che avevano dato lustro alla disciplina attraverso la dottrina e l'insegnamento scolastico, collocati in ordinata rassegna alfabetica dal celebre Antonio da Budrio (1360 ca.-1408) al meno noto Ubertino Zuccardi, originario di Correggio, docente di diritto a Ferrara e morto nel 1541.

In questa silloge non manca un breve medaglione dedicato a Niccolò Mattarelli, più scarno di quello tracciato dal Diplovatazio e con elementi in comune, in specie l'allusione al ricordo personale dichiarato da Giovanni d'Andrea, collega di Mattarelli a Padova per alcuni anni, e la mancata disponibilità dei commentari scritti dal maestro modenese, di cui Marco Mantova afferma di non conoscere esemplari dati alle stampe ${ }^{28}$. Nel novero della tradizionale e affermata dottrina trecentesca si osserva pure il rinvio alla presunzione di Baldo nell'approfondire con maggior acutezza una materia già trattata da Mattarellii ${ }^{29}$, mentre una citazione meno risalente fa rife-

28 Marci Mantuae, Epitoma virorum illustrium, f. 51r: «Nicolaus Materellus Johannis Andreae fuit contemporaneus, quod ex eo constat, quia simul in quadam causa sibi ad consulendum commissa hic colloquium habuerunt, ut ipse Johannis Andreae testatur in addit. Spec. 1. ad fin. in $\S$ sequitur videre de confess. Docuit hic rursum Bononiae et Pisis, multa etiam scripsit commentaria nunquam tamen quod ego viderim edita, et etiam consilia eoque argumento licet etiam videre, quia Baldus, ut suo loco diximus, gloriatur in suis 439 incip. Amplius in fine vol. 3 scripsisse fusius in quadam materia quam ipse Materellus fecisset, quem novissime citat Cuma. Consi. XXXII incip. inculpatus in fine». La citazione di Baldo riprende quanto già ricordato nel medaglione a lui specificamente dedicato (f. $17 \mathrm{v}$ ): «Gloriatur [Baldus] se plenius in materia quadam scripsisse quam fecerit alias Nicolaus Materellus, consi. CCCCXXXIX, incip. Amplius, in fi. vol. 3». Viene poi integrato il profilo di Mattarelli nella serie finale di 'addenda', laddove si precisa (f. 66r): «In Nicolaum Materellum, quod in levi puncto erravit Bald. in 1. fi. in $\S$ si autem C. de iure delibe.». Sull'opera di Mantova si rinvia alla scheda di CocheTTI, Repertori bibliografici del Cinquecento, p. $53 \mathrm{~s}$.

29 Baldi Ubaldi, Consiliorum sive responsorum liber tertium, cons. 439, f. 127vb, n. 13: «Ultimo scias, quod Nicolaus Matarellus disputavit de materia ista, sed de gratia ego meliora et sapidiora dixi vobis». Il confronto con Mattarelli viene puntualizzato al termine di un consiglio in materia di avocazione di feudi in Regno Apuliae, in chiusura dell'ultima sezione dedicata ad analizzare le quattro cause determinanti il trasferimento della giurisdizione. Il consilium ha stretti rapporti contenutistici e compositivi con la quaestio disputata sul medesimo tema da Baldo a Padova nel 1379 e copiata all'interno del ms. 264, ff. 210vb-215vb, della Biblioteca del Collegio di Spagna: Maffei, I codici del Collegio di Spagna di Bologna, p. 717 s. Come già segnalato da BESTA, Baldo e la storia letteraria del diritto, p. 96 s., uguale 
rimento alla raccolta di consigli prodotti dal giurista comasco Raffaele Raimondi (1377-1427), pubblicati nel 1490 a cura del bresciano Giovanni Domenico Patusio frammisti a quelli del più noto Raffaele Fulgosio $^{30}$.

Dal ricordo che Marco Mantova dedica a Mattarelli derivandolo - almeno in parte - dalla perduta opera di Giovanni Battista da Sambiagio ${ }^{31}$, emerge come elemento nuovo l'individuazione di Bologna e Pisa quali sedi del suo insegnamento. Si tratta in realtà di riferimenti problematici, che possono essere stati introdotti surretiziamente al solo scopo di accrescere la statura professionale del maestro modenese legandola a due sedi universitarie di indubbio

richiamo viene orgogliosamente espresso dal maestro perugino in BALDI UBALDI, In VII, VIII, IX, X et XI Codicis libros Commentaria, f. 175ra, n. 29 (ad C. 8.48.1), ove ancora si ricordano le cause che determinano la perdita della giurisdizione, anche se in tal caso in ambito ecclesiastico: «Et no. quod quatuor causae sunt dissolvendi iurisdictionem. s. appellatio, nullitas, negligentia, in Auth. de questione, § si vero aliquis, item mors in casibus in iure espressi de haer. C. ad abolendam, per Inn. Ultimo scias quod in hac materia disputavit Nic. de Ma. sed ego Dei gratia utiliora dixi et sapidiora [...]». Tale giudizio di Baldo è ricordato da BeLlomo, Le istituzioni particolari e i problemi del potere, p. 212, nota 18, richiamando la quaestio di Mattarelli nota al maestro perugino. La presunzione di esporre la materia specifica in modo più perspicuo e conciso Baldo non si astiene dal manifestarla, con le medesime parole, neppure riguardo al proprio venerato maestro, Bartolo: si vedano esempi in Montorzi, Fides in rem publicam, p. 322 s. e in Quaglioni, Il pubblico dei legisti trecenteschi, p. 183.

30 Raphaelis Cumani nempe et Fulgosii, Consilia sive responsa, cons. XXXII, f. 20v. Profilo biografico più aggiornato in VALSECCHI, Raimondi Raffaele, p. 1650: la raccolta di consilia conosce altre edizioni a Pavia nel 1508, a Trino nel 1521, a Lione nel 1548, a Venezia nel 1576, a Francoforte nel 1613. Si vedano pure BeLLoNI, Professori giuristi a Padova, p. 311 ss., e CORTESE, Il diritto nella storia medievale, II, p. 451 e nota 160.

31 Che Mantova abbia avuto tra le mani il perduto De studio legali adipiscendo composto da Giovan Battista da Sambiagio nell'avanzato secolo XV si deduce confrontando i contenuti del profilo dedicato a Mattarelli da Marco Mantova con le pur scarne note apposte da Antonio Porcellini, docente presso l'Università di Padova nei primi anni del secolo XVI, a margine del nome del giurista modenese registrato nella Matricola del Collegio padovano dei Dottori: «Dominus Nicolaus de Mattarellis de Mutina legum doctor. D. Nicolaus de Materellis et D. Johannes Andreae semper contemporanei fuere, ut colligitur in additus (sic) Spec., ti. de confessis, legit D. Nicolaus Bononiae, Pisis, Paduae. Edidit pulcras lecturas supra ff. et cod., licet rara sit copia: ut dixit d. Baptista de Sancto Blasio [in] suo tractatu de studio legali adipiscendo» (ANDrich, Glosse di Antonio Porcellino, p. 10; vedi anche sopra, nota 2 e sotto, nota 126 ). 
prestigio al tempo dell'autore stesso ${ }^{32}$. In ogni caso, allo stato delle conoscenze odierne non sussiste alcuna documentazione né coeva a Mattarelli né posteriore in grado di avvallare la sua attività di insegnamento in queste due città.

Un'unica testimonianza in grado di riflettere un legame tra il giurista modenese, già prestigioso docente, e lo Studium felsineo si ritrova in una supplica formulata e presentata a Taddeo Pepoli, in qualità di conservator civitatis et studii Bononie, dal rector universitatis ultramontanorum nomine suo et vice omnium scolarium per sollecitare una decisione in merito alla contestata elezione di un insegnante di diritto civile per gli anni successivi; tra i nomi dei docenti usciti dall'elezione figura anche quello del dominum Nicolaum in congnomine de Matarollis (sic), ma l'elettore che esprime questo voto dichiara in seguito di essersi sbagliato e di convergere, unendosi agli altri tre elettori, nell'esprimere la sua preferenza per dominus Tomax de la Fossa ${ }^{33}$. Il documento, privo di data, in base ai riferimenti interni è attribuibile al tardo inverno o alla primavera dell'anno 1339, quando tuttavia Mattarelli è defunto da tempo come pure il figlio Francesco, che muore prima del $1336^{34}$, vanificando con ciò ogni possibile legame con il mondo dell'insegnamento universitario bolognese, mai attestato neppure da fonti più risalenti.

Anche di una presunta attività di docenza a Pisa svolta da Mattarelli è arduo trovare conferme documentarie. Da parte di Marco

32 Anche a Baldo viene falsamente attribuito un periodo di insegnamento a Bologna dopo aver conseguito il dottorato, mentre nella città felsinea egli tenne soltanto una lezione pubblica e solenne nel 1370: CORTESE, Il diritto nella storia medievale, II, p. 438; ID., Baldo degli Ubaldi, p. 149.

33 Documento trascritto in Rodolico, Dal Comune alla Signoria, p. 285 s., n. 83, privo di data ma attribuito al tardo inverno-primavera del 1339, richiamato anche a p. 190. Non si evidenziano tuttavia elementi e neppure labili indizi per giustificare l'errore compiuto in sede di votazione di un docente gradito agli scolari bolognesi, dal momento che erano trascorsi oltre vent'anni dalla documentata scomparsa del maestro modenese. Il ruolo attribuito a Taddeo Pepoli si inquadra nell'esperienza signorile da lui avviata a partire dal 1337, a conclusione di una precedente fase di dominazione papale esercitata sulla città di Bologna tramite il cardinale legato Bertrando del Poggetto: si veda il lungo profilo dedicato a Taddeo e al periodo della sua signoria in SARTI-FATTORINI, De claris archigymnasii bononiensis professoribus, I, pp. 276-286; più di recente AnTOGnOLI, Conservator pacis et iustitiae, p. 125 ss.

34 VICINI, Di Niccolò Mattarelli, p. 28. 
Mantova, un legame tra la sua esperienza tanto di studente quanto di apprezzato insegnante presso lo Studio padovano, che mai volle abbandonare a fronte anche di inviti a tenere cattedra altrove, e l'università di Pisa si può ritrovare unicamente nei dialoghi su temi giuridici che egli pubblica nel 1553 dedicandoli al granduca Cosimo I de' Medici, a dieci anni di distanza dalla riapertura dello Studio pisano da lui promossa ${ }^{35}$. Un'operazione tutta intessuta di grata riconoscenza per l'invito, ricevuto da Mantova tramite il nobile fiorentino Leonardo Nasi, a entrare nel novero dei docenti del rinnovato Ateneo toscano e da lui declinato nonostante le honestissimae conditiones propostegli; riconoscenza estesa alla restaurazione della facoltà giuridica che, legandosi a una tradizione risalente al terzo decennio del secolo XII e a una cultura romanistica già all'epoca utile alla formazione dell'élite di governo della città ${ }^{36}$, si rinnova in forma stabile a partire dai primi decenni del Trecento, quando l'Ateneo pisano decolla realmente chiamando a tenere cattedra nel $1338 \mathrm{Ra}-$ nieri Arsendi, già maestro di Bartolo a Bologna nei primi anni Trenta, e l'anno seguente lo stesso, giovane Bartolo, che vi rimarrà sino a tutto il $1343^{37}$.

Nella prospettiva, già aperta dal Diplovatazio, di ricostruire la storia della scienza giuridica medievale partendo dalle biografie e dagli scritti degli stessi autori si colloca l'opera più nota di Guido Panciroli (1523-1599), pubblicata postuma nel 1637, che si giova tanto dell'approfondita cultura giuridica del suo autore,

35 Bassanellus. Mar. Mantui, Bonauiti, Patauini, iuriscon. Caesareique comitis minimi, Colloquia, f. 2r-v. Su questa fase di rinascita dell'Ateneo pisano si veda Marrara, L'età medicea, p. 79 ss.

36 Osservazioni e bibliografia in tal senso in STоRTі Storchi, Intorno ai Costituti pisani, p. 13; CORTESE, Il diritto nella storia medievale, II, p. 13, nota 13, con richiamo alla nota missiva inviata da un monaco vittorino in cui egli chiede al proprio superiore il permesso di trasferirsi a Pisa ad exercendum ibi studium (DuFOUR-GIORDANENGO-GOURON, L'attrait des 'leges', p. $528 \mathrm{~s}$. per il testo della lettera, riportata in modo convincente agli anni 1124-1127 rispetto a precedenti datazioni oscillanti tra il 1065 e il 1213). Si ricorda pure la formazione di apparati interpretativi ai Constituta pisani, frutto di un approfondimento scientifico legato alla prassi giudiziaria locale e dovuto, nel tardo Duecento, a una schiera di magistrati attivi nei tribunali cittadini: CORTESE, Il diritto nella storia medievale, II, p. $295 \mathrm{s.}$

37 Spagnesi, Il diritto, p. 191 ss.; Cortese, Il diritto nella storia medievale, II, pp. 415, 427; Lepsius, Bartolo da Sassoferrato, p. 177; Belloni, Bartolo studente e maestro, p. $561 \mathrm{~s}$. 
apprezzato docente di diritto civile a Padova e a Torino, quanto dei suoi larghi interessi nel settore bibliografico ed editoria$\mathrm{le}^{38}$. In essa Panciroli compendia fortemente il profilo di Niccolò Mattarelli seguendo la traccia del Diplovatazio e appoggiandosi a brevi testimonianze desunte dalle opere di Giovanni d'Andrea e di Cino $^{39}$. Sottolinea, sulla scia del predecessore, l'interesse di Mattarelli verso il ius civile e la disputa di quaestiones, ma sposta troppo in avanti, dal punto di vista cronologico, la sua attività di docenza a Padova agganciandola in modo puntuale al 1314, anno in cui egli risulta invece già defunto ${ }^{40}$. $\mathrm{Al}$ pari di Marco Mantova riferisce di insegnamenti tenuti da Mattarelli a Bologna e quindi a Pisa - per i quali, come già precisato, non sussistono riscontri in altre fonti - e concorda con il giurista padovano sul fatto che di quanto abbia scritto, nulla sia stato rinvenuto. In modo invece originale allude a un ultimo incarico giudiziario svolto da Mattarelli a Lucca, città ove egli avrebbe concluso la propria vita; in ciò prendendo assai verosimilmente spunto da notizie già circolanti a Modena alla metà del secolo XVI e cristallizzate nella cronachistica cittadina del tempo, cui il reggiano Panciroli potrebbe avere

38 A Torino, ove insegna dal 1570 al 1582, Panciroli cura per il tipografo Niccolò Bevilacqua l'edizione dei Commentaria al Codice e al Digesto di Giason del Maino (1573) e quindi i Consilia e i Commentaria di Bartolo (1577); nei successivi anni padovani presta opera di consulenza per la Bibliotheca selecta (1593) di Antonio Possevino e in particolare per la grande collezione veneziana dei Tractatus Universi Iuris (1583-86) curata da Giovan Battista Ziletti: MATTONE, Manuale giuridico e insegnamento del diritto, in part. par. 8, integrato dalla recente voce biografica di Rossi, Panciroli (Panzirolus) Guido; sulle opere di Panciroli soprattutto pubblicate postume si veda SERRaI, Storia delle bibliografia, II, p. 183 ss. Un aspetto particolare della cultura erudito-antiquaria coltivata da Panciroli si riflette in un'opera inedita dedicata alla numismatica antica, identificata presso la Biblioteca Reale di Torino e studiata da Missere Fontana, Guido Panciroli numismatico.

39 Guidi Panziroli, De claris legum interpretibus, p. 181 (ed. Lipsiae 1721, p. 146): «Bononiae Cino et Ioanne Andree profitentibus (a: Io. And. in addi. Ad specul., in tit. de confess. § sequent.), Nicolaus Mattarellus mutinensis an. MCCCXIIII Patavii docebat, ubi diu ius civile interpretatus (b: Cin. in 1. I, col. 9, post n. 15, C. de sent., Qui pro eo, quod interest. et in 1. 1, an. n. 2, C. de confess), etiam quaestiones disputavit (c: Io. And. in addit. Ad specul. de locato, $\S$ id videndum in ver. prim.); postea Bononiae, deinde Pisis leges explicuisse dicitur, in quas etsi multa scripsit, nihil tamen reperitur. Ferunt eum postremo praetorem Lucae ius dicentem in magistratu decessisse».

$40 \mathrm{Si}$ veda cap. I, paragrafo 9. 
attinto senza eccessive difficoltà anche tramite conoscenti diretti o corrispondenti locali ${ }^{41}$.

La notorietà acquisita da Panciroli, benché non sempre pari all'affidabilità dei dati e delle informazioni distribuite nella sua opera biografica, rimane comunque elevata al punto che nella sua analoga collana di biografie giuridiche, una delle più ricche e conosciute prodotte dalla cultura francese in età moderna anche se in base a uno schema unicamente compilativo e non scevra da errori, Pierre Taisand (1644-1715) ripropone, traducendolo letteralmente, il medaglione di Niccolò Mattarelli confezionato dal giurista reggiano oltre un secolo prima ${ }^{42}$.

\section{Un genere nuovo: la bibliografia giuridica}

Dagli inizi del Cinquecento - come ben noto - nel campo della produzione del libro giuridico inizia a prendere corpo il settore specifico della bibliografia giuridica, che conosce uno rapido e significativo sviluppo per rispondere alle esigenze di orientamento nel mare magnum della letteratura connessa a questo specifico ambito culturale e professionale, che dagli esordi della stampa aveva conosciuto una diffusione già notevolissima tramite la pubblicazione di autori sia di rilievo sia di secondo piano e di opere delle più varia specie $\mathrm{e}$ consistenza ${ }^{43}$.

${ }^{41}$ Cronaca modenese di Tommasino de' Bianchi detto de' Lancellotti, XI, pp. 143, 151, con riferimento all'anno 1552; PANinI, Cronica della Città di Modona, p. 77. Tommasino de' Bianchi muore nel 1554 e Francesco Panini dichiara di comporre la sua Cronaca ancora nel 1567. Entrambe non riferiscono di un presunto incarico giudiziario svolta da Mattarelli a Lucca, ma unicamente della presenza nella città toscana di esemplari dei suoi scritti.

42 TaISAND, Les vies des plus celebres jurisconsultes, p. 365. La valutazione della sua opera si deve a SAvigny, Storia del diritto romano, I, p. 499. Taisand, avvocato e poi Tesoriere di Francia, redige anche una storia del diritto romano, edita a Parigi nel 1678, e un importante commentario del diritto consuetudinario praticato nel ducato di Borgogna, pubblicato a Digione nel 1698: per un primissimo orientamento si rinvia al profilo bio-bibliografico di PAPILLON, Bibliothéque des auteurs de Bourgogne, pp. 305-308 e a PetiTJean, Taisand Pierre.

43 Un primo avvio di un censimento complessivo delle edizioni giuridiche del secolo XVI è stato presentato da OsLer, Towards a legal-historical Bibliography. 
A tali obiettivi cerca di rispondere un succinto ma fondamentale testo dato alle stampe a Lione nel 1522 da Giovanni Nevizzano: l'Inventarium librorum in vtroque iure hactenus impressorum, articolato secondo le partizioni tradizionali dei due Corpora Iuris seguite da un'ampia rassegna su base tipologica della produzione dottrinaria di diritto comune, che si configurava, pur nella sua stringatezza, come un utilissimo strumento di consultazione bibliografica sia per il tecnico del diritto, sia per il libraio interessato al commercio di quegli stessi testi ${ }^{44}$. Nonostante arrivasse a citare circa un migliaio di opere a stampa di diritto civile e canonico, l'Inventarium non era comunque esaustivo giacché lo stesso Nevizzano, in chiusura, si dichiarava consapevole di non avere avuto notizia di altri plures [...] libros impressos né di avere perlustrato plures tam ecclesiasticas quam mercatorias bibliothecas disseminate in molte città italiane.

Soltanto tre anni dopo l'opera è pubblicata in versione accresciuta per cura di Luis Gómez ${ }^{45}$ e mezzo secolo più tardi essa viene inserita, come parte iniziale, nell'Elenchus omnium auctorum sive scriptorum, qui in iure tam civili quam canonico [...] clarue-

Sulla produzione di repertori bibliografici relativi allo specifico settore del diritto a partire dal tardo XV secolo si rinvia a SERRAI, Storia della bibliografia, III, p. 438 ss. e alla più recente rassegna ragionata di MATTONE, Manuale giuridico e insegnamento del diritto, in part. par. 8 (Edizioni di fonti e repertori e nuove aperture disciplinari).

${ }_{44}$ L'Inventarium, che si può considerare la prima bibliografia giuridica pubblicata in forma autonoma, è organizzato secondo le seguenti tipologie testuali: Textus et lecturae in iure civili et feudis, Textus extravagantes, Textus et lecture in iure canonico, Doctores in practica, Singularia et cautelae, Repertoria, Contrarietates, Decisiones, Repetitiones in iure civili, Repetitiones in iure canonico, Tractatus diversi, Disputationes diverse, Consilia diversa et extravagantia, cui seguono due quaestiones: Questio quia oportet habere plures libros, Quomodo posset resecari tanta librorum multitudo. Si veda Serrai, Storia della bibliografia, III, p. 438 s. e la breve scheda in CochetTI, Repertori bibliografici del Cinquecento, p. $31 \mathrm{~s}$. Con la sua opera Nevizzano tenta un primo bilancio «di mezzo secolo di una nuova era», ossia quella segnata dalla riproducibilità meccanica del testo giuridico, che ne ha determinato il veloce e larghissimo successo editoriale: SpagnesI, Introduzione, p. VIII.

45 Gómez, Index librorum omnium qui in vtroque iure hinc inde eduntur. Si veda Serrai, Storia della bibliografia, III, p. 441, assieme alla breve scheda in CoCHETTI, Repertori bibliografici del Cinquecento, p. 33. A differenza di Nevizzano, Gómez integra la corposa bibliografia tramite l'indicazione sia di opere manoscritte, specificando talvolta la biblioteca di appartenenza, sia di successiva pubblicazione. 
runt, composto dal giurista ed erudito bavarese Johann Wolfgang Freymon (Freymonius, 1546-1610) e dedicato a Johann Fichard ${ }^{46}$, il quale in Francoforte gli aveva messo a disposizione la propria biblioteca e i propri materiali per permettergli di completare l'imponente Symphonia juris utriusque cronologica, considerata la sua opera più importante ${ }^{47}$. In quel contesto Freymon poté redigerne anche un'altra a carattere bibliografico sviluppando l'Index omnium librorum in iure tam pontificio quam civili pubblicato dallo stesso Fichard accrescendo di corpose aggiunte l'elenco di Giovanni Nevizzano già integrato da Gómez ${ }^{48}$. Se nella più ampia rassegna della bibliografia giuridica messa a punto da Fichard - come si vedrà meglio in seguito - non si rinvengono tracce di testi attribuiti a Niccolò Mattarelli, qualche debole memoria emerge invece nelle opere del medesimo genere pubblicate successivamente da Freymon.

Già la Symphonia, negli elenchi conclusivi organizzati su base cronologica, colloca Nicolaus Mutinensis fra i iurisconsulti recentiores legandolo alla data del $1338^{49}$, che tuttavia manca di riscontri nella biografia del giurista modenese, già defunto nel 1314, e può soltanto avvicinarsi a quella genericamente riportata nella cronachistica cittadina basso medievale, che ne fissa la maggiore fioritura all'anno 1334 collegando la sua produzione dottrinaria in specie al

46 FREYMON, Elenchus omnium auctorum sive scriptorum qui in iure tam civili quam canonico [...] claruerunt (con edizione accresciuta pubblicata sempre a Francoforte nel 1579 e 1585). Per un primo orientamento sulla figura e l'opera complessiva del giurista tedesco si vedano i profili di StintZing-Oefele, Freymonius, Johann Wolfgang; Merzbacher, Freymon von Randeck, Johann Wolfgang; CochetTI, Repertori bibliografici del Cinquecento, p. 80 s.; SerRaI, Storia della bibliografia, III, p. 444.

${ }_{47}$ FREYMON, Symphonia juris utriusque chronologica, strutturata secondo vari elenchi: la sequenza degli imperatori romani (da Cesare a Giustiniano) con le costituzioni rispettivamente promulgate; la lista dei consoli con l'elenco delle leggi emanate sotto i rispettivi consolati; una serie di circa 80 giuristi la cui dottrina è confluita nel Digesto; l'elenco dei decreti papali inseriti nel Corpus Iuris Canonici e una nomenclatura finale di papi, imperatori e giuristi estesa fino al secolo XVI. Si veda CochetтI, Repertori bibliografici del Cinquecento, p. $130 \mathrm{~s}$.

48 Indice leggibile all'interno delle Iurisconsultorum vitae, veterum quidem, per Bernardinum Rutilium. Sull'opera bibliografica di Fichard si rinvia a SERRAI, Storia della bibliografia, III, p. $441 \mathrm{~s}$.

49 Freymon, Symphonia juris utriusque chronologica, p. 915. 
Codice e al Digesto e sempre ribadendo la scarsa disponibilità, già avvertita al tempo, di esemplari delle sue opere ${ }^{50}$.

Nell'Elenchus omnium auctores di diritto civile e canonico pubblicato per la prima volta da Freymon nel 1574 a Nicolaus de Maturellis sono attribuite letture super omnes partes ordinarias iuris civilis tam matutinas quam vespertinas, secondo un riferimento generico e onnicomprensivo alle diverse parti del Corpus Iuris oggetto di lezioni ordinarie, ovvero Codice e Digesto, che non trova poi alcuna conferma all'interno delle analitiche partizioni che, nelle pagine successive del medesimo Elenchus, danno conto della produzione dottrinaria articolata in base alle suddivisioni canoniche dei libri legales $^{51}$. Opere a stampa attribuite a Mattarelli sono completamente assenti pure nello sviluppo della copiosa serie di categorie in cui viene ulteriormente suddivisa la letteratura giuridica sia di ambito civilistico che canonistico, così come all'interno dei fitti elenchi di raccolte di Consilia, Disputationes e Quaestiones sviluppati in base ai nomi dei rispettivi autori ${ }^{52}$.

Il giurista modenese è parimenti ignorato nell'Index nominum omnium interpretum compilato da Giovan Battista Ziletti in chiusura dell'opera di Freymon, così come nella pregevole storia del diritto romano, dotata di una lunga serie finale di medaglioni bio-bibliografici dei maestri che si sono succeduti da Irnerio fino al maturo secolo XVI, data alle stampe a Basilea nel 1565 dal giurista tedesco Valentin Forster (1530-1608) ${ }^{53}$.

Al catalogo di Freymon farà un cursorio riferimento, sullo scorcio del secolo successivo, Agostino Fontana all'interno del suo vastissimo e noto repertorio dedicato alla scienza giuridica ordinato

50 Vedi sopra cap. II, nota 2.

51 FreYmon, Elenchus omnium auctorum sive scriptorum qui in iure tam civili quam canonico [...] claruerunt, f. 13r. Rimane assai improbabile l'identificazione di Mattarelli con un imprecisato N. Manuscriptus ricordato da Freymon come autore di letture sulla seconda parte dell'Inforziato (f. $17 \mathrm{v}$ ), sulla prima parte del Digesto nuovo (f. 18v) e sulla seconda parte del Codice (f. 22r), e ancora come autore di una Repetitio sulla 1. societatem, del titolo Pro socio (C. 4.37.1), ma inclusa nell'incerta categoria dei Fragmenta rerum varia (f. 34r: N. manusc. in Bib. Anto., ovvero nella biblioteca di Antonio Agustin). Lo stesso autore anonimo viene associato anche a repetitiones di ambito canonistico (f. 49r, f. 51r)

52 Ibidem, f. 101r ss., f. 116r ss., f. 117v ss.

53 Valentini Forsteri Iurisconsulti, De historia Iuris Civilis Romani libri tres. 
sia per autore che per soggetto, supponendo Mattarelli originario di Napoli e ricordando pure sue rade citazioni sia da parte del giureconsulto romano Prospero Farinacci, sia all'interno della additiones ai consilia criminalia di Bartolomeo Bertazzoli ${ }^{54}$.

Dopo gli ampliamenti all'Inventarium di Giovanni Nevizzano realizzati da Gómez, Fichard e Gesner alla metà del Cinquecento $0^{55}$, l'opera del giurista alessandrino conosce le ulteriori integrazioni apportate da Giovan Battista Ziletti nel suo Index librorum omnium nomina complectens in utroque iure tam pontificio quam caesareo (apparso a Venezia in quattro edizioni tra 1559 e 1566), che articola con ulteriori suddivisioni le classificazioni tipologiche predisposte da Nevizzano, allarga la rassegna bibliografica a circa un migliaio di segnalazioni e predispone indici alfabetici di autori e opere per guidare in modo ragionato la consultazione del proprio repertorio ${ }^{56}$.

54 Fontana, Bibliothecae legalis amplissimae continuatio, parte VI, col. 160: «Mattarelli (Nicolai) (Neapolitani, ni fallor) Lecturae super Digest. relat. a Freymonio pag. mihi 12 [...]. Mattarellis [Nicolaus] refertur a Farinaccio in tractatu de falsitate, q. 153, n. 138 et 141. Et quod Baldus usus fuit familiariter commentariis eiusdem Nicolai de Mattarellis asserit Bertazolus, Consilia criminalia 172, in Addition.». Per il riferimento ai consilia di Bertazzoli vedi sotto, nota 98; per quello al trattato di Farinacci vedi Farinacci, Variarum quaestionum et communium opinionum criminalium liber quintus, p. 70, nn. 138, 139, 141: i richiami a Mattarelli sono desunti dalla lettura di Baldo al IV libro del Codice, in specie ad C. 4.21 De fide instrumentorum, per cui si veda sotto, nota 75. Sulla figura e l'opera di Fontana si rinvia a SARTI, Fontana Agostino; su Farinacci a Del Re, Farinacci giureconsulto romano (1544-1618), largamente ripreso da Mazzacane, Farinacci Prospero, anche per i riferimenti alle edizioni delle sue opere. Un profilo originale della sua produzione scientifica, per il quale il giurista romano era già largamente noto ai contemporanei, viene approfondito da Schlosser, Prospero Farinacci (1544-1618), ein bedeutender Kanonist?

55 Gesner riserva la partizione XIX del primo dei due volumi delle sue Pandectae (Pandectarum sive partitionum universalium Conradi Gesneri Tigurini), alle scienze giuridiche suddividendolo in tre indici, tra i quali i primi due riproducono quelli messi a punto da Johann Fichard e da lui pubblicati a Basilea nel 1539: SERRAI, Storia della bibliografia, II, pp. 301 ss., 389 ss., e già p. 209 ss. per la complessiva analisi della vita e dell'opera dell'autore zurighese; CochetтI, Repertori bibliografici del Cinquecento, p. 42 s.; Colli, Le edizioni dell'Index librorum omnium iuris civilis et pontificii di Giovanni Battista Ziletti, p. 209 s.; SABвA, La 'Bibliotheca universalis' di Conrad Gesner, p. 19 ss. per il profilo biografico e intellettuale del prolifico bibliografo svizzero e p. 37 ss. per il progetto editoriale e la composizione delle Pandectae, strettamente correlato a quello della Bibliotheca Universalis stampata sempre a Zurigo tre anni prima, nel 1545.

56 Colli, Le edizioni dell'Index librorum omnium iuris civilis et pontificii $d i$ Giovanni Battista Ziletti, in specie p. 210 ss., oltre alla succinta scheda di CocHETTI, Repertori bibliografici del Cinquecento, p. 62. 
In questo indice a Nicolaus de Mutina, nel quale assai verosimilmente si può riconoscere Mattarelli, sono attribuiti generici Commentaria al Digesto e al Codice ${ }^{57}$, anticipando nella sostanza il richiamo leggibile nella bibliografia che sarà pubblicata nel 1574 da Freymon, il quale ricorda il maestro modenese - come detto sopra come autore di sole letture sulle parti riservate alle lezioni ordinarie di diritto civile.

\section{Altri percorsi della bibliografia germanica}

A dieci anni di distanza dall'Epitoma virorum illustrium di Marco Mantova vengono pubblicate, sempre a Padova, le Vitae recentiorum iurisconsultorum ${ }^{58}$ composte da Johann Fichard (1512-1581) e originariamente impresse a Basilea nel 1539, prima opera tedesca dedicata alla storia della letteratura giuridica redatta dall'autore come prosecuzione delle Vitae veterum jurisconsultorum date alle stampe nel 1536 dal giurista Bernardino Rutilio (1504-1538), ripubblicate a Roma nel 1536 e poi a Lione nel $1538^{59}$ e apparse anche l'anno successivo a Basilea in edizione comune con l'opera di Fichard $^{60}$.

Nato a Francoforte e attivo come avvocato e procuratore legale presso il Reichskammergericht in Spira, Fichard svolge anche incarichi diplomatici rappresentando la propria città nei contatti con corti principesche e altre città tedesche in occasione di varie diete imperiali. Tra l'aprile del 1536 e l'autunno del 1537 egli compie un lungo viaggio in Italia al seguito della corte imperiale che gli consente dapprima di incontrare Andrea Alciato a Pavia e quindi di

57 Colli, Le edizioni dell'Index librorum omnium iuris civilis et pontificii $d i$ Giovanni Battista Ziletti, p. 243.

58 FICHARD, Vitae recentiorum iurisconsultorum.

59 Rutilio, Iurisconsultorum vitae Bernardino Rutilio auctore. Su Rutilio e la sua opera, dedicata alle vite di 86 giuristi romani intrecciate con la narrazione delle contemporanee vicende storiche e politiche, si veda STINTZING, Geschichte der deutchen Rechtswissenschaft, I, p. 592 s., assieme alla breve scheda di CосHЕTтI, Repertori bibliografici del Cinquecento, p. 35.

60 CochetтI, Repertori bibliografici del Cinquecento, p. 36 s. 
perfezionare i propri studi a Padova, ove soggiorna per nove mesi, dal dicembre 1536 al settembre successivo frequentando le lezioni dell'ormai anziano Mariano Sozzini, figlio di Bartolomeo, e di Giovanni Antonio Rossi da Alessandria, ricordato come virum quidem stupendae memoriae, oltre a quelle di Lazzaro Bonamici in humanioribus litteris ${ }^{61}$.

Nel corso del suo suavissimum litterarum ocium patavino Fichard ha modo di entrare in stretto contatto con l'ambiente accademico ove Marco Mantova tiene cattedra ormai da un ventennio e di maturare - come emerge dalle date di stampa della sua opera - uno specifico interesse per i principali esponenti della scienza giuridica espressi dall'età medievale e dalla prima età moderna e per una sistemazione dei rispettivi profili biografici in forma enciclopedica che egli estende sino all'età a lui contemporanea. Il testo, che verrà stampato per la prima volta a Basilea nel 1539, si conclude infatti, dal punto di vista della progressione cronologica, con il medaglione dedicato a Ulrich Zasius, del quale Fichard era stato allievo a Freiburg, lo Studio ove egli si era laureato in diritto nel 1532 dopo avere studiato anche ad Heidelberg e Basilea ${ }^{62}$.

61 Tra 1537 e 1542 Fichard compone una autobiografia, cui dà il titolo di Descriptio brevis cursus vitae meae, pubblicata per la prima volta dal «Frankfurtischen Archiv für ältere deutsche Literatur und Geschichte», 2 (1812), pp. 3-53: si vedano p. 22 s. per il ricordo dell'esperienza universitaria e p. 29 ss. per i riferimenti al viaggio in Italia. A quest'ultimo, nella forma di dettagliato itinerario geografico-culturale di forte impronta umanistica e con un'ampia parte dedicata alla città di Roma, il giurista di Francoforte dedica un'opera monografica nella quale ricorda oltre 100 località da lui visitate, dai valichi alpini sino alla zona di Napoli e dei Campi Flegrei, che egli intitola Italia e che viene data alle stampe quasi tre secoli dopo in «Frankfurtischen Archiv für ältere deutsche Literatur und Geschichte», 3 (1815), pp. 3-130: si veda in specie p. 129 per la breve descrizione della città di Padova, nel cui contesto egli rammenta il proprio soggiorno collocandolo tuttavia tra il novembre 1536 e l'agosto 1537 . Su questa particolare e intensa esperienza vissuta da Fichard, che rappresenta una delle primissime testimonianze di un itinerario di formazione culturale sulle tracce della classicità sparse nella Penisola compiuto da un intellettuale di origine nordica, si vedano gli studi recenti di Sünderhauf, Wissenstransfer zwischen Deutschland und Italien; Ead., Von der Wahrnehmung zur Beschreibung.

62 Un'ampia biografia del giurista tedesco, noto anche per avere realizzato una consolidazione del diritto civile patrio e avere dato alle stampe un volume di Consilia, si deve a JuNG, Dr. Johann Fichard, 1512-1581; profili più riassuntivi sono curati da Stintzing, Fichard, Johann von; Friederichs, Fichard, Johann von; Winter- 
In essa Fichard non riserva un profilo specifico all'antiquus doctor Niccolò Mattarelli, ma ne fa emergere brevemente il ricordo in chiusura di quello assai ampio dedicato a Bartolo ${ }^{63}$ nel momento in cui sottolinea la memoria non abbastanza ferma e precisa che avrebbe caratterizzato il maestro di Sassoferrato - come già evidenziato dal Diplovatazio ${ }^{64}$ - nella citazione sia dei passi delle leggi romane sia delle opinioni di altri giuristi, al punto di farsi aiutare da Francesco Tigrini ${ }^{65}$ nel corretto reperimento dei primi e di omettere, invece, in maniera più disinvolta il nome dei secondi, con il facile risultato di attribuire a se stesso il pensiero altrui. Una operazione che Bartolo avrebbe condotto - sempre a detta di Fichard - saccheg-

BERG, Fichard Johann; KLEINHEYER-SchrÖDER, Deutsche Juristen aus fünf Jahrhunderten, p. 89 ss.; SchEmBs, Fichard; KARG, Fichard Johann. Il rapporto di discepolato nei confronti di Zasius viene rimarcato da WIEACKER, Privatrechtsgeschichte der Neuzeit, p. 156 s. In Fichard si può anche riconoscere l'autore di un'apprezzata Ars notariatus, pubblicata anonima a Francoforte nel 1539 e diffusa tramite numerose ristampe veneziane e lionesi sino agli inizi del Seicento: SinISI, Formulari e cultura giuridica, p. 37 ss.; ID., Alle origini del notariato latino, pp. 197 s., 211 ss.

63 FIchard, Iurisconsultorum vitae, p. 240 s.: «Diplovatacius autem hoc de Bartholo commemorat, quod memoriam non satis firmam fidelemque habuerit, adeo ut si aliquid commmentaretur, neque leges in promptu reperiret, Franciscum Tigri (sic) pisanum (qui eodem tempore legit Perusii et ipsi intima amicitia erat coniunctus) solitus fuerit accedere, qui locos illi indicarit et leges suppeditaverit. Habebat enim hic Franciscus magnam singularium rerum memoriam, ut de ipso Baldus refert. Et sane Angelus quoque et Castrensis testantur, Bartholum ex dictis Francisci Tigrini magnum sibi honorem comparasse, exiguum autem ipsi Francisco, ut quem aut numquam aut raro nominaret. Reprehenditur autem Bartholus alias quoque hoc de vitio, quod similiter veterum quoque nomina tacuerit et illorum inventa sibi sumpserit, praecipue autem Nicolai de Maturellis (sic) mutinensis antiqui doctoris, cuius permultas opiniones sibi ascripserit. Sed ita profecto res habet, quod nullum tam excellens perfectumque ingenium fuit unquam, quin suos habuerit aemulos et osores (sic); et eo quidem plures infestioresque quo ipsum est caeteris praestantius [...]». Con minime varianti formali il testo è leggibile anche nella posteriore edizione inclusa in Guidi Panziroli, De claris legum interpretibus (ed. Lipsiae 1721), pp. 397-434, a p. $413 \mathrm{~s}$. L'opera di Fichard è censita pure nel vasto repertorio della dottrina e della storia giuridica di ZiLETTI, Index librorum omnium, f. 32v.

64 Diversamente, SAvignY, Storia del diritto romano, I, p. 495, sottolinea che fonte principale di Fichard sarebbe Caccialupi.

65 Sul maestro pisano (1303 ca.-ante 1365), docente di diritto a Perugia tra 1345 e '55, si veda Peruzzi, Prime note, oltre alla voce biografica a cura del medesimo Peruzzi, Tigrini (Tigrino) Francesco, e quella in Autographa, I.1., pp. 64-65 (voce curata da G. Murano). La collaborazione fra Tigrini e Bartolo è confermata nei manoscritti da consilia rilasciati assieme dai due giuristi: Lepsius, Bartolo da Sassoferrato, p. 178. 
giando in misura consistente le opere di Mattarelli ma che tuttavia non rappresenta un'eccezione nel modus operandi dei commentatori bassomedievali, avvezzi a costruire teorie appoggiandosi con una disinvoltura sin'anche eccessiva alle opinioni dei predecessori fornendo così materia per una tradizione di accuse e rimostranze che si prolunga nei secoli ${ }^{66}$.

Trova così eco nella letteratura biografica del Cinquecento la sostanziale accusa di plagio a danno del giurista modenese che accompagna la fama di Bartolo già negli scritti di alcuni noti maestri dei due secoli precedenti, anche se su basi non sempre fondate. Nell'ambito della lettura al IV libro del Codice Baldo denuncia il fatto che il proprio maestro, citando l'opinione di Mattarelli nel commento alla l. nuda ratio del Digesto Nuovo (D. 39.5.26), avrebbe omesso di specificarne l'autore originario ${ }^{67}$. E anche nella lettura al VI libro del Codice egli ribadisce l'appropriazione del pensiero di Mattarelli di cui si sarebbe reso colpevole Bartolo, che lo stesso Baldo senza mezzi termini arriva a qualificare con l'epiteto di fur, come pure dopo di lui Giason del Maino68,

66 «Vetus querela et criminatio in viros magnos», come la definisce ancora nel Settecento Facciolati, Fasti Gymnasii Patavini, II, p. XXXV, in relazione proprio all'esperienza di Mattarelli. Le osservazioni di Fichard circa i furti dottrinari operati da Bartolo ai danni di Mattarelli trovano eco pure nella letteratura erudita di ambito perugino: Vermigliol, Biografia degli scrittori perugini, p. 24.

${ }_{67}$ BaLdi Ubaldi, In quartum et quintum codicis libros commentaria, f. 37va, n. 3, ad C. 4.19.6: «[...] et ista sunt illius (scil. Nico. de Mata.) notabilia que verba Bar. authorem supprimens refert $[\ldots] »$.

68 ID., In sextum codicis librum commentaria, f. 52ra, n. 8 (ad C. 6.20.13): «Et quia Nic. de Mat. scripsit hic quaedam, quae Bar. sibi attribuit ideo ut antiquorum laus non lateat, de verbo ad verbum hic transcribendum duxi, qui sic ait»; f. 84va, n. 59 (ad C. 6.26.8): «Attende tu, nam haec verba fuerunt antiqui docto. Nic. de Mata. unde aut Bar., qui utitur hoc sermone, fuit fur, aut in duobus corporibus fuit una forma intellectus». Passi già segnalati in MeIJERs, Etudes d'histoire du droit, III, p. 286. Ma si veda pure BALDI UBALDI, In sextum codicis librum commentaria, f. 97vb, n. 57 (ad Auth. Ex causa, post C. 6.28.4): «Et nota quod Bart. istam distinctionem sibi appropriat, que tamen non est sua, sed. Nico. predicti hic in sua lectura. Bart. tamen latius accipit dictam distinctionem quam Nico., quia eodem modo distinguit in legatis et fideicommissis particularibus [...]»; f. 127ra, n. 21 (ad C. 6.36.8): «Sed Nic. de Mata (cuius opinio attribuit sibi Bar.) distinguit inter scientem et ignorantem [...]». Uno dei passi sopra ricordati viene ripreso letteralmente da Giason del Maino nella lettura al Digesto nuovo, ribadendo un'accusa che non gli impedisce di considerare Bartolo come «un riferimento quasi imprescindibile» né di attirarsi, a propria volta, l'analoga accusa di plagio per la dipendenza che manifesta nei confronti delle opere di giuristi più risalenti: IASONIS MAYNI, In secundam digesti novi partem commentaria, $\mathrm{f}$. 
benché sul punto specifico, inerente le sostituzioni ereditarie in età pupillare e le formule da impiegare, Andrea Padovani non ravvisi «alcun elemento di novità potenzialmente ignoto a Bartolo, né una reale coincidenza di vedute col maestro di Sassoferrato», sottraendo così molto credito alla troppo facile accusa mossagli dal suo allievo perugino ${ }^{69}$. Il quale, d'altro canto, oltre a rinnovare attestati di profonda stima e sincera ammirazione verso Bartolo $^{70}$, in diversi passaggi delle proprie letture non lesina testimonianze analoghe nei confronti di Mattarelli e di apprezzamento nei confronti della sua opera, fatta oggetto di copiosissimi richiami nell'ambito dell'esegesi del IV e del VI libro del Codice giustinianeo ${ }^{71} \mathrm{e}$ talora riproposta alla lettera al fine precipuo di non occultare - a detta dell'autore - l'ingegno dei grandi uomini ${ }^{72}$.

163vb, n. 38 (ad D. 45.1.132): «[...] quia Bartolus in multis tacuit veterum nomina et sibi applicavit ingenium aliorum, quod fiendum non est, ubi laus recte quaeritur, et quia Bartolus ascribit sibi opiniones Nicolai de Mattarellis mutinensis antiqui doctoris, unde aut Bartolus fuit fur, aut in duobus corporibus fuit una forma intellectus»; Di Renzo Villata, Giasone dei Maino, p. 997 s. per la citazione.

69 Padovani, Studi storici sulla dottrina delle sostituzioni, p. 274 s.

70 Esempi della grata memoria di Baldo verso il proprio maestro in MonTorzI, Fides in rem publicam, p. 322.

71 Una particolare espressione di ammirazione pare cogliersi in Baldi Ubaldi, In quartum et quintum codicis libros commentaria, f. 54vb, n. 24 (ad Auth. Sed et si quis, post C. 4.20): «Sed Nico. de Mat. procedendo cum suo intellectu, ultra omnes alios dicit $[\ldots] »$. Anche in diversi altri passi viene esplicitato il consenso verso l'opinione di Mattarelli: ibidem, f. 37ra, n. 1 (ad C. 4.19.5); «[...] sed Nic. de Ma. melius intellexit [...]»; f. 78rb, n. 2 (ad Auth. Si qua mulier, post C. 4.29.22): «[...] quare vera est opinio Nico. de Mat.»; f. 80ra, n. 12 (ad C. 4.30.3): «[...] et plenius explicat Nico. de Ma. hic [...]»; f. 102ra, n. 12 (ad C. 4.35.22): «[...] tunc est vera opinio Nic. de Mata»; f. 118rb, n. 2 (ad C. 4.45.2): «Tamen quia ista materia est utilis et male declarata, Nic. de Mat. hic eam examinat per aliquas quaestiones et primo quaeritur [...]»; f. 122ra, n. 5 (ad C. 4.49.4): «[...] ut hic eleganter notat Nic. de Mat. [...]». Baldi Ubaldi, In sextum codicis librum commentaria, f. 58vb (ad C. 6.22.8): «[...] et super hoc Nico. de Mat. dixit multa verba et quia clare loquitur, ideo ea non pono formaliter [...]» Per alcune di queste citazioni si veda anche TAMASSIA; Baldo studiato nelle sue opere, p. 14 e BESTA, Baldo e la storia letteraria del diritto, p. 92, i quali tuttavia non riferiscono che una minima parte delle allegazioni baldesche del pensiero di Mattarelli sparse nelle Letture del maestro perugino. Con una scelta notevolmente diversa, conseguente al modesto interesse che Mattarelli sembra rivolgere alla materia feudistica, nella sua Lettura ai Libri feudorum Baldo ne allega una sola volta l'opinione: Baldi lectura feudorum, f. 78va [n. 16; L.F. 2.53, § Iniuria punitur]: sul punto si veda MonTorzI, L'«intellettualismo pratico» della Lectura super usibus feudorum, pp. 231, 261, e già DANusso, Ricerche sulla «Lectura feudorum», p. 57.

72 BaLdi UBALDI, In quartum et quintum codicis libros commentaria, $\mathrm{f}$. 81rb, n. 15 (ad Auth. Contra qui propriam, post. C. 4.30.3): «[...] secundum Nic. 
Baldo, per di più, fa riferimento con precisione sia, e più volte, a una lectura di Mattarelli - verosimilmente allo stesso Codice $^{73}$ - sia a un suo apparatus $^{74}$ e pure a un suo cursus lectionum ${ }^{75}$, ovvero a testi scolastici del docente modenese che egli avrebbe potuto avvicinare direttamente nel corso del suo prolungato soggiorno padovano, protrattosi per oltre tre anni tra la primavera del 1376 e l'autunno del $1379^{76}$. E tramite l'opera di Baldo sporadiche opinioni risalenti al

de Mat. cuius fuerunt predicta quasi de verbo ad verbum»; f. 86rb, n. 15 (ad C. 4.31.4): «[...] secundum Nic. de Mat. cuius fuerunt plurima ex dictis, sed non omnia»; f. 105ra, n. 20 in fine (ad C. 6.30.19): «Sed hoc latius superius explicavi, dicta autem verba Nic. formaliter refero, ut nihil antiquitatis ignoretur, ne occultetur ingenia magnorum virorum, quibus furtim multi detrahunt». Un'alta opinione del maestro modenese appena attenuata dal rilievo in merito a un unico «levissimo punto» ove anch'egli «caruit intellectu»: ibidem, f. 111rb, n. 2 (ad C. 6.30.22.14).

73 BaLdi UBALDI, In quartum et quintum codicis librum commentaria, $\mathrm{f}$. 19r, n. 1 (ad C. 4.10.1); ID., In sextum codicis librum commentaria, f. 6ra, n. 17 (ad Auth. Sed novo iure, post C. 6.1.3); f. 17ra, n. 33 et in fine (ad C. 6.2.3); f. 25va, n. 1 (ad C. 6.7.2); f. 38rb, n. 1 (ad C. 6.11.2); f. 38va, n. 4 (ad C. 6.11.2); f. 41rb, n. 26 in fine (ad C. 6.14.3); f. 41vb, n. 28 (ad C. 6.14.3); f. 46rb, n. 5 (ad C. 6.18.1); f. 46vb, n. 7 et in fine (ad Auth. Praeterea, post C. 6.18.1); f. 52rb, n. 11 (ad C. 6.20.13); f. 97vb, n. 57 (ad Auth. Ex causa, post C. 6.28.4); f. 127rb, n. 26 (ad C. 6.36.8). Nel commento alla 1. qui se patris (C. 6.14.3), l'adesione di Baldo alla tesi di Mattarelli in tema di revocabilità dei privilegi concessi dal principe è sottolineata da CORTESE, La norma giuridica, I, p. 162, nota 32. Un probabile riferimento a una lectura di Mattarelli emerge pure da un consilium in materia processuale formulato da Oldrado: Valsecchi, Oldrado da Ponte e i suoi consilia, p. 312, nota 886 .

74 BaLdi Ubaldi, In sextum codicis librum commentaria, f. 168rb, n. 18 (ad C. 6.47.1).

75 ID., In quartum et quintum codicis librum commentaria, f. 63va, n. 16 (ad C. 4.21.20).

76 Scalvanti, Notizie e documenti sulla vita di Baldo, Angelo e Pietro degli Ubaldi, p. 210 s.; Colli, Le opere di Baldo, pp. 61 ss., 73 ss.; Cortese, Baldo degli Ubaldi, p. 149 s.; Murano, Baldo degli Ubaldi, p. 104 s.: nel 1379 Baldo pubblica a Padova la sua lectura al VI libro del Codice, benché mancante della parte conclusiva relativa a C. 6.41-62, e negli anni immediatamente seguenti, una volta rientrato a Perugia su richiesta degli organi politici della città, giunge a completare, tra varie opere, anche le letture ai libri I-III, al IV e al V libro del Codice. Si tratta di testi non compiutamente stabilizzati a livello della prima redazione, come emerge dalle ricerche di Vincenzo Colli: la Lettura al VI libro conosce un completamento successivo all'originaria pubblicazione padovana e pure quella al IV libro circola con ampliamenti posteriori, come pure le letture ai primi tre libri, testimoniati soltanto da una parte della tradizione manoscritta. Anche relativamente alla lettura al V libro del Codice «si ha l'impressione che anche in questo caso il testo definitivo, accolto dal- 
maestro modenese da lui ampiamente recepito raggiungono perfino un predicatore francescano comunque attrezzato nelle leges romanae come Giovanni da Capestrano (1386-1456), il quale ne rilancia l'eco nel suo Tractatus contra cupiditatem et avaritiam, nato come approfondimento in chiave giurisprudenziale di una serie di prediche tenute a Verona nel $1438^{77}$.

\section{Esempi di bibliografie universali e settoriali}

Rimane un dato ben consolidato nelle principali opere di storiografia giuridica del maturo Cinquecento la mancata disponibilità di scritti riconducibili a Mattarelli. Per quanto, dal punto di vista dottrinario, sia considerato un punto di riferimento ancora da maestri del Tre e Quattrocento, i suoi testi non giungono a beneficiare del rilancio garantito dalla potente macchina editoriale che, con il suo baricentro italiano in Venezia ${ }^{78}$, si concentra nella diffusione del testo giuridico attingendo a piene mani nel vasto bacino della più diffusa e autorevole dottrina elaborata in età bassomedievale. Di lui e della sua attività di insegnamento permangono generici ricordi legati alla quasi scontata produzione di Lecturae sui testi portanti della formazione giuridica degli studenti, ossia Codice e Digesto - come rammenta Caccialupi - che vengono facilmente dilatati all'intero orizzonte del Corpus Iuris nella maggior parte delle testimonianze prodotte all'interno del nuovo e fortunato genere della bibliografia a stampa, tanto di respiro universale quanto di specifico interesse

le edizioni, sia il risultato di un accrescimento progressivo e di successive revisioni da parte dell'autore» (Colli, Le opere di Baldo, p. 73). Nel corso degli anni trascorsi a Padova Baldo mette a punto anche delle repetitiones, come quella alla 1. Edita actio (C. 2.1.3) divulgata anche nei commentari a stampa e presente in un codice della raccolta Maffei, ivi copiata da Guido di Angelo da Castiglione: MAFreI, Baldo e Alberto Guidalotti fra Perugia e Padova con altre notizie, p. 23.

77 Favino, Giovanni da Capestrano ed il diritto civile, in part. pp. 261, 276.

78 Rimane indiscusso il primato veneziano nella produzione libraria del secolo XVI, in specie di area giuridica, cui si affianca una consistente attività di stampa e di scambio commerciale del libro di legge in altri centri come Trino e Pavia: si veda in merito Nuovo, I Giolito e l'editoria giuridica del XVI secolo; Ead., Produzione e circolazione di libri giuridici tra Italia e Francia. 
giuridico. Un genere che può essere esplorato almeno per apices allo scopo di verificare la qualità delle informazioni in grado attestare la produzione scritta di Niccolò Mattarelli e di valutare meglio l'impatto dell'irruzione della stampa sulla mancata trasmissione della sua opera dottrinaria.

Al pari di Giovan Battista Caccialupi, dal quale dimostra fortemente di dipendere nel delineare il breve profilo di Mattarelli, sullo scorcio del Quattrocento l'agostiniano Giacomo Filippo Foresti, «il primo allestitore moderno di bibliografie impresse ${ }^{79}$, ribadisce in forma piattamente tralatizia sia la scrittura da parte del giurista modenese di accurate letture su Codice e Digesto, sia la rarità di testimoni di esse ${ }^{80}$. Supponendo di identificare queste ultime con i testi qualificati nel secolo precedente come Decisa da Guglielmo da Pastrengo e Alberico da Rosciate, se ne potrebbe giustificare il naufragio a causa dello scarso interesse manifestato dalla Scuola verso la conservazione e la diffusione di opere a prevalente destinazione didattica, prive - come si può dedurre - di sufficiente originalità e comunque più limitate, per sviluppo e approfondimenti, rispetto alle più complete Letture odofrediane. Opere che appaiono rapidamente superate e di non particolare utilità nella formazione giuridica delle successive generazioni, anche per il fatto di essere in misura più o meno variabile rifluite - come dimostra il Tractatus super instrumentis conservato in un unico codice vaticano e largamente reimpiegato da Giovanni d'Andrea ${ }^{81}$ - in opere di

79 Serrai, Storia della bibliografia, I, p. 31 e p. 42 ss.

80 [Iacobi PhilipPI Bergomensis], Supplementum chronicarum: tracciando un breve profilo storico di Modena segnala l'origine locale di Mattarelli, unico testimone della storia e dell'identità culturale della città padana citato da Foresti: «Habuit [scil.: haec civitas] etiam Nicolaum Matarellum iurisconsultum, et bonarum literarum ornatum, cuius clara extant doctrinae monumenta» (l. VI, f. 105v); in seguito, dopo Giovanni d'Andrea, Cino e Oldrado, viene ricordato ancora Mattarelli: «Nicolaum autem Mutinensem superioribus viris nequaquam in legibus doctrina inferiorem, et ipse hisdem temporibus et auctoritate et scriptis floruisse ferunt. Qui et ipse in Digestis et Codice solemnes, et accuratas conscripsit lecturas, de quibus rara habetur copia» (1. XIII, f. 249v).

81 Mattarelli, Tractatus super instrumentis, p. 10 ss.: «i due terzi circa dell'opera di Mattarelli si trovano ripresi nelle Additiones di Giovanni d'Andrea al grande Speculum iudiciale di Guglielmo Durante», in particolare all'interno del titolo de instrumentorum editione della II parte del II libro. Vedi anche sopra, cap. II, par. 8. 
altri giuristi affermatesi con una più larga circolazione e con maggior successo.

Con l'opera di Foresti, nato in territorio bergamasco nel 1434 ed entrato a neppure vent'anni nell'Ordine Agostiniano, ci collochiamo nel solco delle storie di respiro universale e di impianto erudito; nata dal proposito di raccogliere, secondo un rigoroso impianto cronologico, tutte le notizie degne di essere tramandate ai posteri e sino ad allora sparse in testi diversi, incluse quelle riferibili ai più illustri esponenti della cultura giuridica dei secoli precedenti e alla bibliografia ad essi riferibile ${ }^{82}$.

Sulla linea tracciata da Caccialupi e Foresti si orientano tutte le successive bibliografie che, a partire da Johannes Trithemius (14621516) e Conrad Gesner (1516-1565), conferiscono uno sviluppo maturo a questo genere letterario, il primo per averne fissato tecnica e metodologia specifica, il secondo per avere cristallizzato in via definitiva tali impostazioni perfezionandole e dando così vita a un catalogo di oltre 5.000 autori affiancati all'elenco delle rispettive opere. Nella sua Bibliotheca universalis Gesner ne segnala oltre 16.000 tra manoscritte e stampate e pertanto il suo grandioso lavoro viene modernamente considerato come «la sola bibliografia universale che sia stata data alla luce dall'invenzione della stampa in poi» ${ }^{83}$.

82 Sull'autore e la sua cronaca universale dalle origini del mondo al 1482, con notizie su vita e opere di circa 750 autori, una metà dei quali compresi tra X e XV secolo, dei quali si danno concisi medaglioni biobibliografici, si veda la scheda di Cochett, Repertori bibliografici del Cinquecento, p. 22, assieme al profilo biografico curato da Megli Frattini, Foresti Giacomo Filippo, anche per l'elenco delle edizioni Quattro-Cinquecentesche delle sue opere, tra le quali il più noto Supplementum. Per le specifiche conoscenze storico-giuridiche del Foresti si rinvia a PADOVANI, The 'Additiones et apostillae super secunda parte Infortiati' of Cinus de Pistoia, in part. p. 154 , nota 6 .

83 Serrai, Storia della bibliografia, I, p. 64 ss. e II, p. 271, da cui la citazione, e CochetTi, Repertori bibliografici del Cinquecento, p. 38 ss., con riferimento a GESNER, Bibliotheca universalis, sive catalogus omnium scriptorum. La Bibliotheca di Gesner è compendiata e integrata con circa 1.800 nuove citazioni bibliografiche da Conrad Wolfhart (Lycostenes) nel suo Elenchus scriptorum omnium, veterum scilicet ac recentiorum, e nuovamente da Josias Simmler in tre opere pubblicate a Zurigo tra 1555 e 1574: Epitome Bibliothecae Conradi Gesneri; Appendix Bibliothecae Conradi Gesneri; Bibliotheca instituta et collecta primum a Conrado Gesnero. Conosce poi una quarta e più ricca integrazione a opera di Johann Jacob Fries (Frisius, 1505-65) nella sua Bibliotheca instituta et collecta. Su questa tradizione bibliografica si veda CochetтI, Repertori bibliografici del Cinquecento, pp. 49 s., 54 
Nel repertorio dell'abate tedesco, apparso a Basilea nel 1494, la figura e l'opera giuridica di Mattarelli, prive di un solido ancoraggio a informazioni dirette e a dati verificabili anche per l'estraneità dell'autore alla tradizione italica, trascolorano in una dimensione di assoluta genericità sia per l'impiego di un registro aggettivale assolutamente di maniera, sia per l'attribuzione al loro autore di una serie di volumina che vengono a coprire l'intero sviluppo di Codice e Digesto secondo la tradizione d'uso e la partizione interna impostati dalla Scuola giuridica medievale. Un'opera dottrinaria di ambito civilistico e di registro scolastico che si era dimostrata utile ai suoi contemporanei contribuendo e renderne noto l'autore ai posteri, ma che non si era espressa tramite contributi di particolare incisività tali da meritare una segnalazione nell'ambito delle bibliografie specifiche di ambito giuridico che si diffondono largamente fra Quattro e Cinquecento in parallelo all'energica crescita del mercato librario nel medesimo settore ${ }^{84}$. Di nuovo errato, inoltre, il riferimento di Mattarelli all'anno 1340, al tempo del contrasto tra Ludovico il Bavaro e papa Benedetto XII, quando ormai il giurista modenese era scomparso da circa un trentennio.

La successiva Bibliotheca Universalis approntata da Gesner non fa altro che recepire pedissequamente il medaglione di Mattarelli predisposto da Trithemius ${ }^{85}$ arricchendolo unicamente con un

ss., 81 s., 88. Su Gesner, oltre alla voce biografica di FuETER, Gesner Konrad, e al saggio di Widman, Nachwort, con ampia bibliografia anteriore sull'autore e la sua attività di biografo e bibliografo e le sue ricerche in ambito naturalistico, si veda la più recente ricerca monografica di SABBA, La 'Bibliotheca universalis' di Conrad Gesner, in part. pp. 28 ss. per ulteriore bibliografia specialistica sul bibliografo zurighese e la sua opera.

84 TRITHEMIUs, Liber de scriptoribus ecclesiasticis, f. 85r-v (ed. Coloniae 1531, f. $108 \mathrm{v}$ ): «Nicolaus Mutinensis vir in utroque iure et in legibus praecipue nobiliter doctus, ingenio subtilis, sermone scholasticus, scientia et consilio cautus, ut posteris ingenii sui monimenta relinqueret. Scripsit in civili iure quaedam praeclara volumina, quibus se presentibus tunc utilem, futuris autem fecerat notum. E quibus feruntur: super Codice, lib. 9; super ff. veteri, lib. 24; super ff. novo, lib. 12; super ff. inforciati, lib. 14; et quaedam alia. Claruit temporibus Ludovici Bavari Imperatoris quarti et Benedicti papae XII. Anno domini 1340».

85 Secondo una dipendenza testuale già osservata da SAvignY, Storia del diritto romano, I, p. 489 s. GESNER, Bibliotheca Universalis und Appendix, I, f. 522r: «Nicolaus de Maturellis Mutinensis, vir in utroque iure doctus, scripsit in civili iure quaedam praeclara volumina, e quibus feruntur: super Codice, lib. 9, super ff. 
nuovo riferimento alla Summa casuum conscientiae, meglio nota come Summa angelica, composta nel tardo Quattrocento dal francescano Angelo Carletti da Chivasso (1414 ca.-1495) con l'intento di predisporre un repertorio alfabetico e una guida nella scelta, in ogni circostanza dell'agire umano, delle soluzioni religiosamente più corrette tramite l'appoggio a un vastissimo repertorio di opere di morale, teologia, biblistica e anche diritto. In essa Nicolaus de Metharelis è ricordato nel corposo anche se non amplissimo elenco iniziale dei doctores utriusque iuris variamente utilizzati come auctoritates nello sviluppo dell'opera ${ }^{86}$.

La rassegna dei più significativi repertori dedicati alla biografia e alla bibliografia giuridica apparsi sul mercato librario tra i secoli XV e XVI, allargata ad analoghe opere orientate al più vasto ma generico ambito della produzione letteraria elaborate sullo scorcio del medioevo e lungo la prima età moderna, conferma che la figura di Mattarelli mantiene uno spazio riconoscibile, anche se appiattito sui profili divulgati dal Diplovatazio e da Panciroli e variamente filtrati in via tralatizia in repertori posteriori.

Non altrettanto si può dire per la sua produzione dottrinaria, che non oltrepassa il tornante dell'invenzione della stampa a caratteri mobili e non rientra nel fiorente mercato delle edizioni giuridiche, cui tanta attenzione presta l'industria editoriale che si sviluppa largamente nel secolo XVI. La circolazione di sue opere in forma manoscritta alimenta la dottrina di rinomati maestri ancora per tutto il

veteri, lib. 24, super ff. novo, lib. 12, super ff. infortiati, lib. 14, et quaedam alia. Citatur in Summa Angelica». Nella Epitome Bibliothecae Conradi Gesneri, Tiguri, apud Christophorum Froschoverum, 1555, ristampata nel 1966 come II parte del II volume di seguito all'Appendix, è ribadito a f. $137 \mathrm{r}$ il medaglione di Mattarelli di poco abbreviato e con riferimento cronologico finale desunto sempre da Trithemius: «Nicolaus de Maturellis Mutinensis, scripsit super Codice lib. 9, super ff. veteri lib. 24, super ff. novo lib. 12, super ff. infortiati lib. 14 et quaedam alia. Citatur in Summa Angelica. Claruit anno 1340».

86 Angelo da Chivasso, Summa angelica, tabula secunda, ad indicem. Sull'autore e la sua opera, risalente al 1486, si rinvia al profilo di Pezzella, Carletti Angelo, unitamente a quello di Schulte, Die Geschichte der Quellen und Literatur des canonischen Rechts, II, p. 452 s. Da ultimo, all'interno del più largo panorama delle summae bassomedievali concepite come guide e ausilii per i confessori, si veda Padovani, Giustizia e lavoro nelle Summae di Antonino da Firenze, Angelo da Chivasso e Giovanni Battista Trovamala, in part. p. 395 ss. 
secolo XIV e si riverbera in quello successivo anche se - come pare - più per l'utilizzo fattone dai commentatori trecenteschi ripresi dai maestri successivi che per diretto accesso da parte di questi ultimi ai testi genuini riferibili al maestro modenese.

Di lui, comunque, non si conoscono in età moderna scritti identificabili con precisione, come conferma ulteriormente verso la metà del Cinquecento il giureconsulto Bernhard Gassner nella sua Nomenclatura doctorum in utroque iure et canonico et civili, concepita come strumento utile a quanti si avvicinano per la prima volta agli studi giuridici e organizzata secondo un elenco alfabetico di circa 300 giuristi in base alle sigle con cui venivano solitamente citati, seguite dal nome completo e dalla frequente segnalazione delle rispettive opere più celebri. Nel caso di Mattarelli, l'unico canale attraverso cui si può riconoscerne e documentarne la dottrina rimangono le poche citazioni tratte da opere di maestri seriori del XIV e XV secolo, unici esempi di una conoscenza indiretta che non viene mai suffragata dal ricordo di opere identificate in modo puntuale, tanto meno diffuse tramite una propria edizione a stampa ${ }^{87}$.

Come prevedibile conseguenza di tale situazione, non compaiono trattati né opere monografiche attribuite a Mattarelli nei notissimi 28 volumi del Tractatus Universi Iuris, come neppure nelle altre raccolte di respiro enciclopedico e di minore portata approntate dall'industria libraria nel corso del Cinquecento ${ }^{88}$. Né emergono alla conoscenza diretta dei giuristi, né più in generale nell'orizzonte della

87 GASSNER, Nomenclatura doctorum in utroque iure et canonico et civili, ad indicem: «Nic. de Mat. id est Nicolaus de Mattarellis, de quo Paul. De Cast. 1. 2, C. de eden., Bart. L. 1, ff. si quis iusdi. non obtem.»; si veda CochetтI, Repertori bibliografici del Cinquecento, p. 38; SERRAI, Storia della bibliografia, III, p. 446 s. Per i rinvii ai commentari di Paolo di Castro e di Bartolo si vedano: PAULUS DE CASTRo, Prima super Codice, f. 38vb, n. 3 (ad C. 2.1.2), ove l'allegazione è mutuata dalle Additiones di Giovanni d'Andrea allo Speculum di Guglielmo Durante; BARTOLI DE Saxoferrato, Prima super Digesto Veteri, f. 56ra, n. 12 (ad D. 2.3pr.), f. 57rb, n. 3 e n. 5 (ad D. 2.3.4). Altrove lo stesso Paolo di Castro ricorda Mattarelli attraverso il filtro di Baldo: Paulus de Castro, Super Codice pars I, f. 252va, n. 1 e f. 253ra, nn. 7-9 (ad C. 4.44.2).

88 Dato che emerge dalla consultazione dei preziosi indici approntati da CoLLI, Per una bibliografia dei trattati giuridici pubblicati nel XVI secolo, I. Indici dei Tractatus universi iuris, e ID., Per una bibliografia dei trattati giuridici pubblicati nel XVI secolo, I. Bibliografia delle raccolte. Su modalità di composizione e rapporti tra queste corpose antologie di trattati, nelle quali si materializza il trionfo del 
cultura dotta, esemplari di altre opere a lui attribuite e riconoscibili con una specifica identità, a partire da letture e commentari elaborati in sede didattica la cui memoria diretta, riflessa nella dottrina di epoca successiva, non oltrepassa comunque il secolo XIV.

Non sorprende quindi trovare conferma di tali lacune nella poderosissima bibliografia giuridica tardo seicentesca approntata da Martin Lipen (1630-1692), che assieme al repertorio enciclopedico messo a punto dal giureconsulto romagnolo Agostino Fontana rappresenta una delle grandi guide bibliografiche del secolo barocco, nuovamente stampata in forma ampliata e corretta ancora nel secolo successivo $^{89}$. In essa manca qualsiasi riferimento a testi attribuiti a Niccolò Mattarelli, mentre emerge la citazione di opere attribuite a Stephanus Materellus e ad Antonius Matharellus ${ }^{90}$, che in realtà si possono ricondurre a due autori francesi del secolo XVI e ai rispettivi scritti: Stephanus Maleretus (Etienne Malleret) e Antonius Matharellus (Antoine Matharel) ${ }^{91}$.

Si può pensare che la grande pestilenza di metà Trecento, aprendo grandi vuoti nella popolazione urbana inclusa quella legata al mondo universitario, abbia reso disponibili notevoli quantità di manoscritti giuridici e li abbia anche abbandonati a una tradizione ma-

genere trattatistico affermatosi progressivamente all'interno della dottrina giuridica circa dalla metà del Trecento, si veda pure Colli, Introduzione bibliografica.

89 Ascheri, Tribunali, giuristi e istituzioni, p. 87 s., ove le opere di Fontana e di Lipen sono ricordate in specie per l'attenzione alle raccolte di Decisiones, laddove il primo «riunisce in un elenco unico le Decisiones dei paesi più diversi», mentre il secondo «ripartisce le raccolte secondo la loro provenienza geografica», distinguendo per l'Italia le raccolte secondo la geografia politica degli stati preunitari. «I due repertori però vanno naturalmente consultati con cautela, tenendo conto che accolgono dati di seconda mano o tramandano errori proprio o altrui e poi, ovviamente, che possono essere incompleti» (p. 88 per le citazioni). Per la figura e l'opera di Agostino Fontana si veda sopra, nota 54.

90 LIPEN, Biblioteca realis iuridica, pp. 176, 225 e p. 155 dell'indice finale: a Stephanus Materellus è attribuito il trattato De electione libera, ad Antonius Matharellus un altro testo indicato sinteticamente Ad Hottomanni Franco-Galliam. La sezione giuridica della Bibliotheca universalis, che include complessivamente oltre 40.000 autori e 130.000 opere, non è che una delle quattro parti in cui si articola l'opera del teologo tedesco oltre alla sezione medica, a quella filosofica e a quella teologica: in merito Serrai, Storia delle bibliografia, IV, p. 414 ss.

91 Stephanus Maleretus (o Malleretus), Ingeniosissima lucubratio viri undecumque doctissimi domini Stephani Malereti; Antonius Matharellus, Ad Franc. Hotomani Franco-Galliam Antonii Matharelli responsio. 
noscritta incerta e meno ordinata del passato, meno sorvegliata tramite il sistema di riproduzione per peciae affermatosi da tempo negli Studia più attivi e prestigiosi come Padova e Bologna ${ }^{92}$. Di qui la sopravvivenza, comunque difficile e incerta, di opere e testi lasciati dai maestri più prestigiosi e ancora richiesti dal mercato, come quelli ascrivibili a Bartolo, e l'implacabile selezione che colpisce invece quelli riferibili a maestri di minor levatura come Mattarelli: al di fuori di un ristretto circuito di specialisti, soprattutto entro il limite più ravvicinato del secolo XIV, esemplari di sue opere non mostrano di avere circolazione né di consolidarsi nelle forme di una tradizione certa, in grado di conservarne una precisa identità e riconoscibilità.

\section{Erudizione locale fra Cinque e Settecento}

Accanto al filone della biografia e della bibliografia giuridica che largo spazio acquista nel panorama editoriale fra i secoli XV e XVI e a quello delle enciclopedie di respiro universale, un percorso significativo è rappresentato dalla letteratura di impianto erudito prodotta in ambito modenese nel corso dell'Età Moderna, che per quanto concerne l'elaborazione della memoria di Mattarelli fa perno sulle opere di Francesco Forciroli, Giovan Battista Scanaroli, Lodovico Vedriani e Girolamo Tiraboschi. I primi imbevuti di spirito municipalistico orientato alla celebrazione delle glorie locali nei diversi settori culturali e professionali tramite una giustapposizione acritica di memorie e notizie diverse; l'ultimo, invece, seriamente attrezzato, sullo scorcio del Settecento, per condurre un esame più maturo delle fonti in grado di delineare un profilo assai più documentato $\mathrm{e}$ compiuto dell'antico giurista modenese ${ }^{93}$.

Si tratta di autori, eccetto Tiraboschi (di natali bergamaschi), che

92 Si richiamano qui le riflessioni formulate in anni recenti da Ascheri, Bartolo da Sassoferrato: introduzione a un giurista globale, p. 1039, e ID., Bartolo da Sassoferrato: il civilista 'globale', p. 18 s., in riferimento al caso del grande maestro marchigiano.

${ }_{93}$ Sulla figura, la formazione culturale e l'intensa produzione letteraria e storiografica dell'abate bergamasco, attivo a Modena dal 1770 nelle funzioni di direttore della biblioteca ducale, si rinvia, tra gli studi degli ultimi decenni, a Di PIETRo, 
condividono con Mattarelli l'origine cittadina, in specie di condizione ecclesiastica, ai quali si devono i primi e organici tentativi di sistematizzare le memorie patrie attorno ad alcuni nuclei tematici omogenei: soggetti accomunati dallo svolgimento di professioni giuridiche - come nell'opera di Vedriani - o dalla più generale appartenenza alla tradizione culturale modenese resa illustre per i meriti più vari - come nel catalogo di Forciroli, inedito fino a tempi recenti - oppure esempi celebri della tradizione letteraria, ricomprendendo in tale categoria tutti gli autori di testi scritti comunque pervenuti a un livello di sufficiente e comprovata notorietà non limitata a un ambito esclusivamente locale - come nella ben nota silloge dell'abate bergamasco. Un discorso a parte interessa invece il testo dottrinario composto dal vescovo Scanaroli, ove la memoria di Mattarelli affiora nell'ambito del ricordo di alcune glorie modenesi ed è sviluppata come ampia digressione a margine del III libro della sua opera dedicata all'assistenza ai carcerati e al funzionamento delle istituzioni preposte a tale ufficio.

Vissuto a cavallo dei secoli XVI e XVII, giureconsulto e auditore a Roma al servizio del cardinale Bonviso Bonvisi (1561-1603) e quindi del cardinale Alessandro d'Este (1568-1624), Francesco Forciroli nell'arco di alcuni decenni si dedica alla compilazione, all'interno di una manipolo di opere storico-biografiche di interesse municipale, pure di una collana di biografie di modenesi illustri rimasta inedita sino a pochi anni or sono ${ }^{94}$.

Nella prima parte degli Illustrium virorum Mutinensium Monumenta, scritti nel corso di molti anni e giunti a noi non in originale, ma in copia settecentesca eseguita dal bibliofilo e collezionista Matteo Pagliaroli, trova posto anche un medaglione dedicato a Mattarelli strutturato come centone di citazioni tratte sia da esemplari della dottrina giuridica, sia da opere di carattere storico-biografico divenute stabile patrimonio della cultura letteraria.

Girolamo Tiraboschi; Venturi, Girolamo Tiraboschi; Mari, Il genio freddo; VentuRI, Girolamo Tiraboschi e le figure dei bibliotecari estensi.

94 Per informazioni sull'autore e le modalità di composizione dell'opera qui richiamata si vedano i saggi introduttivi di Sonia Cavicchioli, Le «Vite»: fortuna di un genere letterario nella cultura del Cinquecento, e di Giorgia Mancini, Note biografiche su Francesco Forciroli, in Forciroli, Vite dei modenesi illustri, pp. 7-20 e 21-24 rispettivamente. 
Alle corpose citazioni tratte dalle opere tre-quattrocentesche di Guglielmo da Pastrengo, Filippo Foresti e Joannes Trittenheim ${ }^{95}$ e a un cursorio richiamo a Caccialupi ${ }^{96}$ si affianca un breve ventaglio di passi sia estrapolati direttamente dalle Letture di Baldo al IV e al VI libro del Codice ${ }^{97}$, sia richiamati, in un solo caso, attraverso la citazione che ne fa Claudio Bertazzoli nelle ampie additiones ai noti Consilia criminalia redatti dal padre Bartolomeo e pubblicati nel $1584^{98}$.

Si osservano anche un paio di riferimenti a fonti documentarie e dottrinarie di ambito modenese con le quali si avvia un primo avvicinamento a materiali maggiormente legati, per produzione e conservazione, alla città di origine di Mattarelli, condiviso da quegli autori che, proprio grazie alle comuni origini o ai più intensi e prolungati rapporti con l'ambiente locale, sono in grado di fare emergere maggiori dettagli sul profilo biografico del giurista. In proposito viene ricordato da Forciroli il commento alla costituzione Habita redatto verso la metà del secolo XVI dal giovane Bernardino Scodobi, allievo di Andrea Alciato ${ }^{99}$, assieme alla matricola duecentesca dei

95 Vedi sopra, cap. I, nota 33; cap. III, nota 80; cap. III, nota 26 e 84.

96 Vedi sopra, nota 8.

97 Richiami assai limitati che a una lettura attenta si possono ampiamente integrare: BALDI UBALDI, In quartum et quintum codicis libros commentaria, f. 35ra, n. 6 e n. 15 e f. 35va, n. 9 in fine (ad C. 4.19); f. 36ra, n. 10 (ad C. 4.19.2); f. 36rb, n. 2 e f. 36vb, n. 18 (ad C. 4.19.3); f. 37va, n. 3 (ad C. 4.19.6); f. 54ra, n. 4, f. 54va, n. 14 , f. $54 \mathrm{vb}$, n. 24 , f. $55 \mathrm{ra}$, n. 27 e n. 31, f. 55rb, n. 37 (ad C. 4.20, Auth. Sed et si quis); f. 55rb, n. 2 in fine e n. 5 (ad C. 4.20.20). Baldi Ubaldi, In sextum codicis libros commentaria, f. 66va, n. 5 e f. 66vb, n. 7 in fine (ad C. 6.23.27); f. 168rb, n. 18 (ad C. 6.47.1). Per un elenco più esaustivo si veda supra, nota 71. Si osserva una concentrazione dei riferimenti al pensiero del maestro modenese nel commento al Titolo 19 (De probationibus), che assieme al 21 (De fide intrumentorum) del IV libro del Codice risulta fortemente correlato ai contenuti del Tractatus super instrumentis di Mattarelli.

98 Bertazzoli, Consiliorum seu responsorum iuris in criminalibus et penalibus controversiis emissorum liber primus, n. 172, additio a f. 235r: «Fuit antiquissima opinio Nicol(ai) de Mattarel(lis), quam refert Baldus in d. rub. C. de fide instrumentorum (ad C. 4.21), et cuius commentariis familiariter usus est ipse Baldus ut circumfertur rumor et hic locus demonstrat». Dopo essersi laureato presso lo Studio ferrarese, Bartolomeo Bertazzoli (1516 ca.-1588) svolge l'ufficio di podestà a Reggio e Modena e quindi, una volta rientrato a Ferrara, si dedica alla professione forense e per un biennio anche all'insegnamento: si veda il profilo di VolANTE, Bertazzoli Bartolomeo.

99 Vedi cap. I, paragrafo 9. 
giudici e degli avvocati modenesi nella quale Mattarelli viene effettivamente registrato e pure al primo posto ${ }^{100}$, anche se l'informazione non è attinta di prima mano, ma riferita dal governatore estense di Finale Orazio Levizzani e appresa da Forciroli in occasione di un soggiorno sul luogo nel 1608.

A tale ventaglio di fonti nulla aggiunge alcuni decenni più tardi Giovan Battista Scanaroli (1579-1665), che nella sua prolissa disamina dottrinaria pubblicata nel 1655 e dedicata non soltanto all'assistenza ai reclusi, ma più in generale al complesso di norme, istituti e prassi che presiedono all'utilizzo della carcerazione tra Cinque e Seicento ${ }^{101}$, trova l'occasione per celebrare la figura del giurista modenese riecheggiando l'errata opinione del Diplovatazio circa il magistero di Mattarelli nei confronti di Bartolo e riportando quasi alla lettera quanto già riferito sul medesimo giurista dal vescovo modenese Gaspare Silingardi (1537-1607) ai primi del secolo XVII ${ }^{102}$.

100 Vedi cap. I, paragrafo 2.

101 La digressione su Mattarelli è sviluppata dall'autore nell'ambito di una parte dedicata alla personificazione della città di Modena e al ricordo, da parte di quest'ultima, di una serie di illustri esponenti della propria tradizione giuridica e culturale. Scanaroli, De visitatione carceratorum libri tres, p. 551: «Iuriprudentiae non displicebit nomen meum audire, quae non ignorat praecipuum agminis sui Bartolum, Nicolai Mattarelli alumni mei, quem saepius allegat, doctrina eruditum, ad tantae laudis fastigium pervenisset. Fuit autem ipse Nicolaus civis meus iurisconsultus celeberrimus et circa anno 1300, dum Patavii publice iura interpretaretur, evocatus ad reformationem status mei, que me in libertatem vidicaveram. Huius scripta gravi studiorum omnium detrimento deperdita sunt, licet aliqui asserant apud rempublicam lucensem asservari. Ita legitur in Catalogo Episc. Mutin. Mutinae anno 1606 impresso sub episcopatu Iacobi anno 1291 in episcopatum electi». Oltre ad alcuni scritti più risalenti di taglio encomiastico (RAGGI, Elogio di Giovanni Battista Scanarolo; Ricci, Di G.B. Scanaroli modenese), sulla figura di Scanaroli, laureatosi in giurisprudenza a Macerata nel 1604 e chiamato a ricoprire dall'anno successivo l'ufficio romano di Procuratore dei Poveri, si vedano più di recente BEDONI, G.B. Scanaroli e L.A. Muratori, e Fornilli, Giambattista Scanaroli, ripreso poi in ID., Delinquenti e carcerati a Roma, p. 111 ss. In chiave di celebrazione venata anche da spirito campanilistico, ne sottolinea le qualità umane e dottrinarie SALA, Della vita e degli scritti di monsignor Giovanbattista Scanaroli. In quanto docente di diritto civile e procedura civile a Modena tra gli anni Sessanta e Settanta del secolo XIX, del prelato modenese Erio Sala ripercorre la carriera romana e ne sintetizza l'opera, che nasce come «commento alla legge sull'istituzione del Tribunale della visita delle carceri e sulla giurisdizione del medesimo [...] diretta a rimediare agli errori e alle ingiustizie degli altri tribunali» (p. 15 s.).

102 Silingardi, Catalogus omnium episcoporum Mutinensium, p. 107: nel contesto del medaglione dedicato al vescovo Jacopo da Ferrara (1290-1331) affiora 
In queste testimonianze trova eco sia l'impegno civile di Mattarelli, richiamato da Padova nella sua città natale per partecipare alle nuove istituzioni comunali cui si cerca di dare vita in seguito alla rivolta antiestense del gennaio 1306, sia la consapevolezza della perdita dei suoi scritti, che una tradizione locale, già affiorante nel secolo precedente attraverso la cronachistica municipale, affermava di avere individuato a Lucca senza che tuttavia si fosse dato seguito al loro effettivo recupero ${ }^{103}$.

Il complesso della memoria di Mattarelli veicolato attraverso il doppio canale della bibliografia storico-giuridica più diffusa e consolidata nei secoli precedenti e dell'erudizione locale si riverbera nel corposo medaglione riservato al giurista modenese all'interno della silloge approntata dopo la metà del Seicento dal sacerdote Lodovico Vedriani (1605-1670), appassionato cronista delle memorie storiche della propria città alle quali riserva, accanto ad alcune monografie di ambito religioso, varie raccolte organizzate per aree tematiche a base prevalentemente biografica ${ }^{104}$, inclusa quella

la memoria pure di Mattarelli: «Hoc sedente episcopo, floruit Nicolaus de Mattarellis mutinensis iurisconsultus celeberrimus, cuius scripta in iure civili magno non solum fama ipsius et nostrae civitatis detrimento, sed etiam gravi omnium studiosorum iactura deperdita sunt, licet aliqui asserant, nescio qua ratione ducti, scripta ipsa in civitate Lucae asservari. Hic egregius doctor hisce temporibus, dum Patavii publice iura civilia interpretaretur, vocatus a civibus suis ad reformandum civitatatis statum, qua se in libertatem vindicaverat, Mutinam venit, ubi statum ipsius reipublicae una cum aliis ad id deputatis optime reformavit». Dopo una prima esperienza come vicario generale della diocesi modenese, Gaspare Silingardi è nominato vescovo di Ripatransone nel 1582 e in seguito ambasciatore, per conto del duca estense Alfonso II, alle corti del re di Spagna Filippo II e di papa Clemente VIII; rientra poi a Modena nel 1593 come vescovo della propria città natale e negli anni successivi è inviato in Francia come nunzio apostolico: oltre alla voce biografica in GazzetTi-Barbieri, Cardinali, vescovi e abati, p. 78 s., con bibliografia anteriore, si veda in particolare HaAn, Correspondance du nonce en France Gasparo Silingardi.

103 Per tali approfondimenti si rinvia al cap. II, paragrafo 8.

104 VedRIANI, Raccolta de' pittori, scultori, et architetti modonesi più celebri; ID., Vite et elogij de' cardinali Modonesi; ID., Vita del glorioso s. Geminiano vescouo; ID., Memorie di molti santi martiri, confessori, e beati modonesi; ID., Vita della beata Camilla Pia; ID., Historia dell'antichissima città di Modona; ID., Catalogo de vescoui modonesi; ID., Breve racconto dell'arma stilata dalla nobilissima famiglia de Cortesi da Modona. Sull'opera biografica e cronistica di Vedriani, oltre alle brevi note di SerRaI, Storia della bibliografia, III, p. 169 s., valutazioni più approfondite si devono a Boccolari, Lodovico Vedriani e la storia di Modena, e a CAPUcCI, Lodovico Vedriani e il biografismo artistico municipale. 
dedicata ai Modenesi distintisi nelle scienze giuridiche, teologiche e filosofiche ${ }^{105}$.

105 VedRIANI, Dottori modonesi, in specie pp. 41-43: «Intorno a gli anni 1300, fiorì nello studio delle leggi Nicolò Mattarelli. Fu di sangue nobile e di famiglia, ch'in ogni tempo ha dato al mondo huomini di valore nelle lettere e nell'armi. Viene questo dottissimo huomo lodato molto da leggisti e da gli historici ancora. Nel tempo che Cino e Gio. Andrea leggevano in Bologna, egli dichiarava in Padova il ius civile. Tra tanti altri scolari, che riuscirono eccellenti sotto gli insegnamenti suoi si nomina Bartolo, il quale dalla dottrina di Nicolò ammaestrato, pervenne a quella gran sapienza e gloria, ch'a tutto il mondo è nota. Anzi è pubblica fama, che Bartolo si appropriasse le dottissime fatiche del Maestro, e vi sono dottori, come si può leggere nella Vita dell'istesso Bartolo, ch'asseriscono lui esser stato di poca memoria, e che commentando qualche luogo non haveva le leggi in pronto e che però era sforzato riceverle altronde, come da Francesco Tigrino Pisani, dal nostro Nicolò e da altri: Nicolai quoque Mattarelli atque aliorum veterum sententias quandoque ad verbum descriptas sibi impudenter attribuisse dicitur et ce. Ciò viene parimenti confirmato dal Panciroli e molto prima da Giasone dottore milanese nella legge Quidam, nel Digesto, sotto il titolo De verborum obligationibus numero 30, ove deplora che tanti dottissimi scritti del detto Nicolò fossero occupati da altri in grave danno della città di Modona e dell'autore. Quante e quante volte cita poi Nicolò nell'opere sue? Si gloria in suis 439 incipit: Amplius in f. scripsisse fussius (sic) in quadam materia, quam ipse Mattarellus fecisset. Mentre leggeva in Padova fu chiamato da Modonesi al governo della patria con altri a tal fine eletti e deputati, la quale vivea allora in libertà, et il tutto ottimamente adempì. Dopo insegnò in Modona, in Bologna et in Pisa. Dalla Biblioteca Gensneriana habbiamo il catalogo delle opere che dottamente scrisse così numerate: Nicolaus de Mattarellis Mut. scripsit super Codice lib. 9, superff. veteri lib. 24, superff. novo lib. 12, super inforciato lib. 14 et quaedam alia. Citatur a Summa Angelica. Claruit 1340, e poco dopo scrive il Gensnero: Nicolaus de Mutina C. super Digestum et Codicem. Da altri scrittori viene detto I.V.D. egregius, i quali affermano ch'egli fece un trattato De testamentorum materia, et alterum De spuriis. Molti autori dicono ch'altre opere di tanto dottore sono appresso la Repubblica di Lucca, della qual città, al sentire del Panciroli, fu podestà e in essa vi morì non havendo ancora terminato il tempo del suo governo. Ma Bernardino Scodobio modonese in quella sua bellissima e utilissima Interpretatione in Autenticam, C. ne filio pro patre, scrive che passò a miglior vita nella patria e che fu sepolto in S. Domenico. Ho letto parte d'una lettera scritta da Lucca del sig. dottore Lodovico Bianchi, il quale era colà in officio, sotto la data del dì 12 di marzo l'anno 1552, che notifica haver veduto tali opere manoscritte, e tocca anche la spesa che si farebbe della stampa, cioè ducatoni 150. Di questo nostro dottore fanno testimonianza il Mantova, car. 52, num. 189, il Silingardi, car. 107, il Panzirolo, lib. 2, cap. 59, car. 181, il vescovo Scanaroli, lib. 3, car. 551, Filippo da Bergamo nelle sue Croniche, cart. Mihi 73, Angelo Portenari nel suo libro intitolato Felicità di Padova, lib. 7, cap. 4, car. 228, il quale nota così: Nicolò Mattarelli da Modona esplicò gloriosamente legge civile in Pisa et in Padova; e altri, che per non esser prolisso passo sotto silentio, i quali lodano i modonesi letterati. Nella sua figura tolta dal naturale osservaranno i lettori ch'anticamente i leggisti portavano il capuccio come segno delle loro professione. Haveva già in Duomo una statua 
All'interno di quest'ultima, giudicata con notevole severità da Tiraboschi quanto a sistematica e affidabilità di alcune tra le biografie ricostruite ${ }^{106}$, il ricordo di Mattarelli è tracciato in buona misura sulla scorta dichiarata delle testimonianze cinquecentesche del Diplovatazio ${ }^{107}$ e di Marco Mantova, grazie alle quali si era già consolidato un profilo identitario della figura e dell'opera di Mattarelli basato essenzialmente sulla sua attività di docenza a Bologna e Pisa, benché mai confortata da specifica documentazione, e sull'impossibilità di reperire suoi scritti; elementi ribaditi in modo pedissequo e acritico da Panciroli e da altri autori i quali, in tempi successivi, si interessano in particolare delle origini e della storia dello Studio patavino ${ }^{108}$.

all'altare de SS. Sadoleti e hora de signori conti Fontana, la quale non si vede più a cagione che l'illustrissimo e reverendissimo signor conte Roberto Fontana, di felice memoria, vescovo di Modona, vi fece fabricare una bellissima cappella e ornarla di finissimi marmi, buone pitture e altre nobili vaghezze».

106 Tiraboschi, Biblioteca modenese, I, p. XVI, e vale la pena di riportare il giudizio del grande bibliotecario e storiografo estense: «Del primo (scil. Vedriani) abbiamo alle stampe i Dottori Modenesi, opera nella quale deesi lodare il buon desiderio ch'egli ebbe di onorar la sua patria, e le molte fatiche, che egli sostenne nel ricercar le notizie al suo disegno opportune. Ma ei non si prefisse di ragionare che dei Dottori di Legge, benché pure ad essi frammischiasse più altri, che non furono mai Dottori, e innoltre ammise nella sua opera tutti quelli, che con quel titolo ei vide distinti, e quelli ancora, che ne' secoli di mezzo aveano in qualche luogo sostenuta la carica di Podestà, supplendo cogli elogi dettati dalla sua immaginazione alla mancanza delle sicure notizie, e non curandosi, secondo l'universal costume d'allora, di darci esatta contezza della vita e delle opere di quelli, che eran degni di più distinta menzione».

107 Da Diplovatazio, circa il presunto discepolato di Bartolo, e da Vedriani, che viene citato in modo esplicito, dipende Lodovico Antonio Muratori nel momento in cui, all'interno del discorso dedicato alla fortune delle lettere in Italia e alle origini di Studia e Accademie, ricorda in poche righe Mattarelli: AIMAe, III, col. 908: «Atque ex hisce mutinensium scholis praeter alios prodiit Nicolaus Matarellus, patria Mutinensis, et Bartolo veluti suus praeceptor saepe laudatus, qui non tantum Mutinae, sed et Bononiae ac Patavii summa cum laude leges interpretatus, libris etiam editis claruit, de quo legendus Vedrianus noster in Libro de Doctoribus Mutinensibus».

108 Per la testimonianza di Panciroli vedi sopra, nota 39. Sulla diretta scia di Panciroli, la cui opera viene stampata soltanto nel 1637, anche Riccoвoni, De Gymnasio patavino, f. 12r: di seguito al breve medaglione dedicato a Oldrado da Lodi, in relazione al 1314, anno presunto della morte di Mattarelli, precisa che «Floruit in Gymnasio patavino Nicolaus Matarellus Mutinensis, qui omnes libros Codicis et pandectarum clarissimis commentariis illustravit et nonnulla alia conscripsit. Horum vitas descripsit Guidus Pancirolus, clarissimus I.C., in libro de claris iuriscon- 
Merita notare che, a fianco di numerose testimonianze - già analizzate in precedenza - riferite in maniera tralatizia, Vedriani trae informazione da non meglio identificati «altri scrittori» circa la stesura da parte di Mattarelli di un paio di trattati dedicati alla materia testamentaria e alla prole illegittima, che così vanno ad aggiungersi al catalogo già abbastanza ricco degli scritti attribuiti al giurista modenese: dalle solemnes et accuratas [...] lecturas su Codice e Digesto ricordate dal Diplovatazio alla riduzione delle letture odofrediane ai medesimi testi giustinianei in compendi noti sotto l'etichetta di Decisa, ben presenti a Guglielmo da Pastrengo nei primi decenni del secolo XIV unitamente a generiche quaestiones e repetitiones sempre riferite a Mattarelli; dalla disputa di numerose quaestiones note attraverso Alberico da Rosciate alle varie opere scolastiche (una lectura, forse al Codice, un apparatus e un cursus lectionum) attribuite da Baldo al proprio predecessore nell'insegnamento del ius civile a Padova. Da Giovanni d'Andrea, inoltre, sappiamo che Mattarelli, suo collega sempre a Padova, sarebbe stato autore di una summa prolixa in materia di instrumenta notarili ${ }^{109}$, che si può identificare con il Tractatus super instrumentis trasmesso mediante un unico manoscritto vaticano valorizzato grazie a una trascrizione recente $^{110}$.

Con la strumentistica a disposizione del dottissimo abate Girolamo Tiraboschi (1731-1794) unita alla sua profonda conoscenza tanto di testi letterari e giuridici ${ }^{111}$ quanto di fonti di ambito modenese-estense, maturata negli oltre due decenni trascorsi a Modena

sultis, nondum edito»; e pure Portenari, Della felicità di Padova, p. 228, ove sempre in rapporto all'anno 1314 ricorda che «Nicolò Matt(ar)elli da Modena esplicò gloriosamente legge civile in Pisa e in Padova».

109 Sorrenti, Testimonianze di Giovanni D’Andrea, n. 83, p. 71.

110 Mattarell, Tractatus super instrumentis; da vedersi assieme allo studio preparatorio, dedicato soprattutto ai contenuti dell'opera, di MACINo, Contributo alla storia della trasmissione del Tractatus super instrumentis. Si veda in particolare sopra, cap. II, par. 8.

111 Un dato che viene rimarcato anche da DionisotTI, Filologia umanistica e testi giuridici, p. 191 s., in relazione alla poderosa Storia della letteratura italiana composta da Tiraboschi in due redazioni (una prima in 10 volumi e 13 tomi, uscita tra 1772 e 1782, con un ulteriore volume di «giunte e correzioni» edito nel 1795; una seconda pubblicata in 9 volumi tra 1787 e 1794), ove trova ampio spazio la storia del diritto tramite una larga scelta di autori di testi dottrinari. 
nelle funzioni di direttore della Biblioteca Ducale quale successore, a vent'anni di distanza, del sommo Muratori ${ }^{112}$, lo spessore del profilo di Niccolò Mattarelli e la ricchezza della documentazione che lo riguarda conoscono un salto di qualità consistente anche se non immediato.

Sfrondandone la biografia da lodi e apprezzamenti eccessivi quanto formali e ingiustificati, non rari nelle testimonianze dei secoli precedenti, nella sua Storia della letteratura italiana Tiraboschi riunisce un primo mosaico di citazioni e riferimenti tratti dalla storiografia erudita di origine, oltre che locale, anche padovana e pisana delineando un sobrio profilo del giurista in grado di fissarne alcune tappe salienti tra Modena e Padova e di confermare l'assenza di sue opere date alle stampe ${ }^{113}$.

Assai più articolata risulta la voce biografica di Mattarelli composta alcuni anni dopo dallo stesso Tiraboschi all'interno della $\mathrm{Bi}$ blioteca modenese grazie a una selezione più ampia di fonti documentarie tratte dagli archivi locali, al loro vaglio critico nel confronto con notizie desunte dalla storiografia anteriore e all'integrazione con citazioni del dottore modenese estratte per consultazione diretta dalla consolidata dottrina di Cino, Giovanni d'Andrea, Giason del Maino e pure da Baldo per il tramite di Marco Mantova.

Accanto alle ben note riduzioni delle Letture di Odofredo si ripropone l'incontrollato catalogo di quelle presuntivamente condotte su Codice e Digesto già rilanciato dalle raccolte bio-bibliografiche quattro e cinquecentesche (Trithemius, Foresti, Gesner), giungendo tuttavia ad ammettere che «niuno ci indica ove tali opere or si conservino» ${ }^{114}$. In merito al problema del reperimento di testi riferibili a Mattarelli, unica traccia significativa aggiunta in chiusura, in comune con la più recente storiografia erudita ravennate ${ }^{115}$, è una citazione tratta dal noto repertorio del maurino Bernard de Montfau-

112 Sul punto specifico si vedano le note di VENTURI, Girolamo Tiraboschi e le figure dei bibliotecari estensi, in specie p. 1056 ss.

113 Tiraboschi, Storia della letteratura italiana, V, p. 243 s. (= G. Tiraboschi, Storia della letteratura italiana, II, Milano 1833, p. 346).

114 ID., Biblioteca modenese, III, pp. 185-190, a p. 189 per la citazione.

115 Ginann, Memorie storico-critiche degli scrittori ravennati, II, p. 46, ove tuttavia non viene dichiarata l'origine di tale citazione. 
con, contenente la sommaria descrizione dei fondi manoscritti delle principali biblioteche europee, secondo la quale presso la Biblioteca Vaticana sarebbero conservati non meglio specificati Tractatus varii di Mattarelli ${ }^{116}$.

Grazie ai cataloghi vaticani compulsati dal dotto monaco francese, un'autorità indiscussa, al tempo, nel settore delle giovani scienze dell'antichità e della ricerca archivistica, affiora per la prima volta all'interno della bibliografia erudita di età moderna un dato preciso in merito all'individuazione di un'opera realmente sopravvissuta nel ventaglio della produzione del giurista modenese ritenuta sino a quel tempo perduta, secondo numerose fonti, oppure giacente ancora a Lucca ma mai esplorata né portata alla luce nella sua consistenza effettiva.

\section{Glorie patrie non soltanto modenesi}

L'attenzione della storiografia erudita di ambito municipalistico nei confronti di Niccolò Mattarelli viene coltivata in età moderna non soltanto da autori connessi in forma più o meno diretta alla medesima patria di origine, ma trova riscontri pure in due altri filoni abbastanza corposi che fanno capo, per ragioni diverse, alla tradizione letteraria di area ravennate e padovana.

Nel primo caso si osserva l'attrazione della figura del giurista duecentesco nel novero delle glorie ravennati grazie all'incrocio di tracce differenti. Da un lato, l'esercizio da parte del figlio, il doctor iuris Francesco, delle funzioni di giudice e vicario del podestà di Ravenna Lamberto da Polenta nell'anno 1313117. Dall'altro, la

116 Montfaucon, Bibliotheca bibliothecarum, I, p. 141: «Nicolai de Mattarellis de Mutina Tractatus varii. 5773». La segnalazione appare corretta: al ms. Vat. Lat. 5773 corrisponde infatti il Tractatus super instrumentis redatto dal maestro modenese, per cui si veda sopra, cap. II, par. 8. Sulla formazione e i caratteri della poderosa opera catalografica approntata dal Maurino francese: Petitmengin, Montfaucon, dom Le Maitre et la Bibliotheca Bibliothecarum.

117 Bernicoli, Governi di Ravenna e di Romagna, p. 33; Vicini, Di Nicolò Mattarelli, p. 28, che trae la notizia da PAsolinI, Lustri ravennati [...] parte terza, libro IX, p. 15. Alla pagina seguente l'erudito ravennate ricorda pure Giovanni Mattarelli, altro figlio di Niccolò, ecclesiastico e docente a Treviso di diritto canonico, il 
dichiarazione di Lodovico Vedriani nel pieno Seicento secondo cui $\mathrm{i}$ «Mattarelli [sarebbero] venuti da Ravenna» ${ }^{118}$, derivata dal fraintendimento di un passo di una cronaca modenese cinquecentesca al tempo disponibile soltanto in forma manoscritta ${ }^{119}$. Sulla medesima scia, ribadendo lo stretto rapporto della famiglia con la città adriatica fin dal secolo XIII, si collocano i migliori esponenti della storiografia locale dai caratteri più schiettamente campanilistici ${ }^{120}$.

Altri esponenti di una omonima famiglia Mattarelli radicata in Ravenna sono comunque conosciuti per i secoli successivi, come Giovanni Battista Mattarelli, docente su cattedre minori nello Studio padovano attorno al $1500^{121}$, e il più noto Achille, giureconsulto al servizio dei cardinali legati nell'amministrazione della provincia ravennate e membro del Senato cittadino, che nel corso del secolo XVII si segnala per avere dato alle stampe alcune pubblicazioni correlate all'amministrazione pontificia e alla disciplina dei rapporti di quest'ultima con la comunità locale in specie in materia di diritti enfiteutici e di interventi sulla rete idraulica del territorio ${ }^{122}$.

$\mathrm{Su}$ un filone diverso, non venato da rivendicazioni di sapore campanilistico bensì saldamente ancorato alla prestigiosa tradizione

quale, annoverato all'interno delle memorie ravennati, viene implicitamente ricompreso fra gli esponenti in qualche modo celebri di quella città. L'ufficio ricoperto da Francesco Mattarelli nel 1313 viene ancor prima ricordato da Rossi, Historiarum ravennatum libri decem, p. 530.

118 VedRIANI, Historia dell'antichissima città di Modona, II, p. 255.

119 Si veda ora nella moderna edizione di PANINI, Cronica della città di Modona, p. 72: nel corso di un lungo elenco di famiglie modenesi esponenti sia del ceto nobiliare che del Populus si ricordano anche i «Sadoleti da Ravenna ove tra le più antiche, e nobili è quella famiglia; Mattarelli; Carretti; Tebaldi; quei dalla Fratta; quei da Castello signori di Spezzano».

120 Pasolini, Lustri ravennati [...] parte seconda, libro VIII, pp. 246-249; ID., Huomini illustri di Ravenna antica, p. 91 s.; GinanNI, Memorie storico-critiche degli scrittori ravennati, II, pp. 43-47: medaglioni bio-bibliografico di Niccolò Mattarelli, il quale - secondo Ginanni - «comunemente dagli scrittori si dice modenese; ciò non ostante appartiene a Ravenna o per nascita o per origine [...]. Onde almeno per la sua origine appartiene a Ravenna» (p. 43).

121 Facciolati, Fasti Gymnasii Patavini, I, p. 68; Belloni, Professori giuristi a Padova, p. 349.

122 Pasolini, Huomini illustri di Ravenna antica, p. 92; Ginanni, Memorie storico-critiche degli scrittori ravennati, II, pp. 41-43. Alcune tra le opere di Achille Mattarelli sono pure censite nel vastissimo repertorio di Fontana, Amphiteatrum legale, parte I, coll. 634, 640. 
culturale cittadina, si devono infine ricordare i principali esponenti della storiografia padovana legata, in specie, alle origini e alla storia dello Studium, che dallo scorcio del Cinquecento fino al secolo XIX rielaborano in varie sedi i profili dei principali maestri di ius civile e canonico senza escludere quello di Mattarelli, documentato per un ventennio a Padova a cavallo dei secoli XIII e XIV con al fianco pure il figlio Francesco, anch'egli doctor iuris.

Sulla scia diretta di Panciroli ${ }^{123}$, all'epoca consultato in forma manoscritta, ne ricorda brevemente il magistero in Padova il letterato e storiografo Antonio Riccoboni (1541-1599), docente di eloquenza a Padova, alla fine del secolo XVI ${ }^{124}$, seguíto in modo ancor più stringato e impreciso da Giacomo Filippo Tomasini (15951655), vescovo di Cittanova d'Istria, alla metà del secolo successi$\mathrm{vo}^{125}$.

Nell'approdo alla storiografia settecentesca si giunge alla pubblicazione di una più corposa storia dell'università di Padova da parte di Niccolò Papadopoli (1655-1740) $)^{126}$ che viene proseguita e in-

123 Per la cui testimonianza si veda sopra, nota 39 e testo corrispondente.

124 Riccoboni, De Gymnasio patavino, ff. 11v-12r: in relazione al 1314, anno presunto della morte di Mattarelli, precisa che «Floruit in Gymnasio patavino Nicolaus Matarellus Mutinensis, qui omnes libros Codicis et pandectarum clarissimis commentariis illustravit et nonnulla alia conscripsit. Horum vitas descripsit Guidus Pancirolus, clarissimus I.C., in libro de claris iurisconsultis, nondum edito». Ignora invece la figura di Mattarelli una precedente silloge dei più illustri esponenti, circa duecento, della cultura padovana approntata dal canonico Bernardino Scardeoni (1478-1574) trascrivendo anche il testo delle iscrizioni funerarie che ne perpetuano la memoria all'interno delle chiese cittadine: De antiquitate urbis Patavii et claris civibus patavinis libri tres, di cui è nota una prima edizione veneziana anteriore di due anni (Venetiis, apud Valgrisium, 1558) difficilmente reperibile. Per la collocazione dell'opera nel contesto del genere bibliografico che tanta fortuna matura nel corso del secolo XVI si rinvia alla scheda di CоснETTI, Repertori bibliografici del Cinquecento, p. 63. Un sintetico profilo biografico in Del Negro, Clariores, p. $278 \mathrm{~s}$.

125 Tomasini Iacobi Philippi, Gymnasium Patavinum, p. 232: in attinenza all'anno 1314 ricorda unicamente che «Nicolaus Matarellus Mutinensis Ius Civile Patavii exponebat et forsitan Paulus Titulus, Nicolaus Plebeius ac Iacobus Antonius Stenuus, sive de Malitia, Patavini I.C. celeberrimi in patrio Gymnasio Ius docuere». Sul letterato ed ecclesiastico padovano si veda il profilo bio-bibliografico in VEDoVA, Biografia degli scrittori padovani, II, p. 334 ss.

126 Papadopoli Nicolal Comneni, Historia gymnasii patavini, p. 197: «Nicolaus Mattarellus, mutinensis, clarus Juris Caesarei professor, quo tempore Cynus et Joannes Andreae Bononiae docebant, ius civile Patavii frequentissimis auditoribus 
tegrata da Jacopo Facciolati (1682-1769) ${ }^{127}$ tramite un lavoro nel complesso rigoroso basato sulla consultazione di innumerevoli documenti, cui egli può accedere grazie al riordino degli archivi universitari da lui compiuto ${ }^{128}$, e anche degli autori locali più risalenti, rifacendosi ai quali risulta tuttavia meno attendibile ${ }^{129}$. Valutate sulla base del medaglione biografico riservato a Mattarelli, docente a Padova per molti anni ma non originario della medesima città, queste opere non si rivelano capaci di staccarsi dall'impronta erudita caratterizzata dall'affastellamento di citazioni derivate da autorevoli

interpretabatur. Ita jurisconsultorm nostrorum alba sive matriculae apud Porcellinum, ex quibus colligimus, collegas habuisse Paulum Titulum, Nicolaum Plebeium et Iacobum Antonium Stennum sive de Malitia, de quo videndus est Pancirolus, jurisconsultos celeberrimos, qui tamen nihil noscendum reliquere posteritati. Ipse Mattarellus, cum diu docuerit Patavii atque etiam disputaverit egregie, teste Cyno, Bononiae quoque, ac Pisis iura fuerit professus authore Joanne Andreae, scripsit multa, quorum pauca supersunt alienis lucubrationibus inserta. Ferunt preturam gessisse Lucae atque in eo magistratu decessisse, signatque hoc ipsum Pancirolus, sed annum emortualem non signat, qui ex coniectura nobis est MCCCXXXIX. De illo Mantua et Caccialupius». Si fa riferimento alle glosse bio-bibliografiche apposte da Antonio Porcellini, professore di diritto civile a Padova e priore del Collegio dei Giuristi nel 1532, accanto ai nomi dei più illustri giureconsulti e docenti registrati all'interno della Matricola che, avviata nel 1435, elenca i dottori aggregati al medesimo Collegio dal 1300 circa al 1640 (Archivio Antico dell'Università di Padova, ms. 134, ff. 1v-15r): si veda in merito Andrich, Glosse di Antonio Porcellino. Su quest'ultimo si rinvia, anche per bibliografia anteriore, a BeLLonI, Professori giuristi a Padova, pp. 321, 335.

127 Facciolati, Fasti Gymnasii Patavini, I, p. 34 s.: «Nicolaus Matarellus Mutinensis inter nostros iuris civilis professores longe hac aetate celeberrimus fuit. Adde iis, quae de illo scribit Papadopolus, legatos a populo mutinensi missos ad civitatem et universitatem patavinam fuisse, ut facultas concederetur sapienti viro $\mathrm{Ni}$ colao de Mattarellis professori legum degendi Mutinae per totum mensem aprilem ad complendum officium Defensoris Populi, ut est in actis mutinensibus. In vetustis Collegii Jurisconsultorum tabulis coniungitur cum Joanne Andreae et alibi quoque docuisse dicitur. Revera docuit in patria, Pisis et Bononiae, multaque scripsit, quae refert Ludovicus Vedrianus de Doctoribus Mutinensibus p. 42. Hoc amplius narrat ex Fichardi fide et publica fama, Bartholum discipulum nonnulla ex eius penu (sic) sumpta et ad verbum descripta sibi attribuisse. Vetus querela et criminatio in viros magnos. Nam ipse quoque Joannes Andreae, tantum vir, insignis alienorum laborum fur nominatur a Baldo». Su Facciolati e sul suo ruolo nell'ambito della storiografia legata alle vicende dell'Ateneo patavino si veda Del Negro, Da Iacopo Facciolati a Francesco Maria Colle, oltre alle brevi note di Boscaino, Facciolati Iacopo, e al recente e sintetico profilo biografico in Del Negro, Clariores, p. $147 \mathrm{~s}$.

128 Boscaino, Facciolati Iacopo, p. 67.

129 Come viene precisato da Belloni, Professori giuristi a Padova, p. 29 s. 
esponenti della biografia giuridica dei secoli precedenti (Caccialupi, Mantova, Fichard) nello sforzo di conferire maggiore spessore al profilo del maestro modenese ma tramite fonti non controllate e ribadite in modo piattamente tralatizio.

Perdura l'assenza di ricerche originali in grado di fare luce tanto sulla sua specifica produzione dottrinaria, quanto sulla documentazione conservata negli archivi delle due città - Modena e Padova - in cui maggiormente si concentra la sua vita e la sua attività professionale; e tale impostazione conferma quei limiti che dimostreranno di saper valicare, almeno in parte, le ricerche dedicate all'ateneo patavino che vedranno la luce nel corso del secolo XIX grazie a Francesco Maria Colle (1744-1815) ${ }^{130}$, laureatosi in giurisprudenza in quella stessa università, e Andrea Gloria (1821-1911) ${ }^{131}$ tramite una meditata e approfondita rassegna della storiografia più risalente e un approccio a fonti documentarie locali in grado di fissare punti certi in merito alle tappe biografiche di Niccolò Mattarelli e pure del figlio Francesco nei rispettivi ruoli di docenti di diritto civile nel prestigioso studio veneto.

Se l'opera di Colle risulta «largamente superiore per quantità e qualità di notizie alle storie dell'ateneo padovano stese in precedenza» ${ }^{132}$, il multiforme impegno di Andrea Gloria nell'esplorare numerosi profili della storia della città di Padova e del suo territorio si indirizza fin da tempi assai precoci verso lo studio dell'istituzione universitaria, del suo patrimonio di manoscritti e della sua memoria storica, sostenendo un impegno che conduce, nel settore

130 Il corposo profilo di Mattarelli, con accenni anche al figlio Francesco, si può leggere in Colle, Storia scientifico-letteraria dello Studio di Padova, pp. 77-84. Tra 1786 e 1789 Colle ricopre l'incarico di storiografo ufficiale dell'Ateneo padovano con il compito di proseguire i Fasti dell'università, lasciati incompiuti da Iacopo Facciolati all'anno 1756, e di stendere un'organica storia della medesima; entrambe le opere verranno pubblicate postume con annotazione integrative dell'erudito padovano Giuseppe Vedova, la prima con il titolo di Fasti Gymnasii Patavini iconibus exornati ab anno MDCCLVII usque ad MDCCCXL (Patavii 1841): PrETo, Colle Francesco Maria.

131 Gloria, Monumenti della Università di Padova, ove si vedano in specie i documenti, tracritti in tutto o in parte, alle pp. 43, 49, 65 s., 66 s., 71.

132 Preto, Colle Francesco Maria, p. 798. 
specifico, alla composizione della sua opera più rilevante ${ }^{133}$.

In tal modo iniziano a emergere solide testimonianze documentarie in grado di accertare alcune tappe significative del ruolo svolto da Mattarelli quale docente presso lo Studio padovano, anticipando di non molti anni il parallelo e assai più ricco contributo monografico dedicato al giurista modenese da parte di Emilio Paolo Vicini, al pari di Gloria laureato in legge, abile paleografo e impiegato presso istituzioni archivistiche cittadine ${ }^{134}$. La più aggiornata scienza dei documenti, basata sulle moderne acquisizioni della diplomatica e della paleografia poste a sostegno delle giovani discipline storiche affermatesi in ambito universitario, rende possibile un più incisivo scavo nella documentazione utile a ricostruire il profilo del giurista, dopo che il pur recente contributo della scienza romanistica di ambito germanico si era limitato a precisarne i contorni attenendosi alle citazioni sparse nella dottrina giuridica e alle opere compilative di impianto erudito ereditate dal passato, in assenza ancora di indagini orientate a portare alla luce testimoni diretti della sua produzione didattica e scientifica ${ }^{135}$.

133 Come sono giudicati da Cerasi, Gloria Andrea, p. 415, i notissimi Monumenti della Università di Padova, 1222-1318, pubblicati a Venezia nel $1884 \mathrm{e}$ concepiti come continuazione del Codice diplomatico padovano, già stampato in due volumi tra 1877 e 1879. Gloria (1821-1911), laureatosi in giurisprudenza nel 1844, si dedica poco dopo alla compilazione del catalogo dei manoscritti della Biblioteca universitaria. Per ulteriori analisi sulle ricerche archivistiche e l'attività dello studioso padovano, docente di paleografia presso la locale università dal 1859 sotto l'amministrazione asburgica e direttore del Museo Civico, si rinvia a GALLo, Università e signoria a Padova, p. 9 ss. e alla relazione presentata dallo stesso Donato Gallo, Andrea Gloria (1821-1911): erudizione municipale e «scienze ausiliarie della storia» a Padova, al convegno Fonti documentarie ed erudizione cittadina. Alle origini della medievistica italiana (1840-1880), Verona, 22-24 ottobre 2015, di prossima pubblicazione on-line in «Reti Medievali-Rivista». Un sintetico profilo biografico in Del Negro, Clariores, p. $176 \mathrm{~s}$.

134 Per i riferimenti a Vicini si veda sopra, Introduzione.

135 Con riferimento al succinto profilo di Mattarelli delineato in SAvigny, Storia del diritto romano nel medio evo, II, p. 453 s., ove le citazioni tratte dagli scritti di Cino e di Giovanni D'Andrea risultano già trasmesse all'interno delle opere degli altri maestri ricordate in nota. 


\section{MANOSCRITTI E DOCUMENTI D’ARCHIVIO}

In corsivo quelli esaminati direttamente o tramite riproduzioni

Alba Iulia

Biblioteca Nazionale Centrale

Biblioteca Centrală de Stat, Filiala Batthyanyaneum

II.I.64: 72

Magl. VI.134: 72

II. $4: 75,76,82$

Magl. XXIX.172: 72

Magl. XXIX.179: 72

Augsburg

Staats- und Stadtbibliothek

FoligNo

2.52: $76,82,83$

Biblioteca Jacobilli

A.VI.17: 95, 98

BolognA

Biblioteca dell'Archiginnasio

B.1393: 97

B.2794: 83

GIRONA

Biblioteca Capitolare

10 [1-2]: 42

Collegio di Spagna

81: 94, 96, 97

La Seu d'Urgell

83: 98

122: 90

Biblioteca Capitular

126: 60

2031: 92

2109: 84, 111

264: 155

LEIPZIG

FIRENZE

Universitätsbibliothek

Biblioteca Medicea Laurenziana

Acquisti e doni $158^{1}: 83$

Lat. 873: 79 
MADRID

Biblioteca Nacional

823: 83, 111

Milano

Biblioteca Ambrosiana

E.25 inf:: 77, 82, 83

Modena

Archivio Capitolare

O.II.11: 14

O.III.30: 14

Diplomatico, pergamene

O.15.670: 50

O.19.690: 50

O.23.709: 50 a. 1293: 31, 51, 74

a. $1306 / 1: 54,61$

a. $1307 / 1: 38,53,61$

a. 1309: 53, 71

a. 1310/1: 63

a. 1314: 54, 63

Archivio Storico Comunale

Camera segreta

I.1: 11,12

I.2: 12

III.1 (Magna Massa Popoli): 55

IV.II.1: 16

Registrum Antiquum: 49

Biblioteca Estense

Lat. 1161 (già a.m.8.19): 4, 88, 89, 90, 91

MÜNCHEN

Bayerische Staatsbibliothek

Clm 26669: 84

Padova

Archivio Antico dell'Università

134: 190

Biblioteca Universitaria

941: 5, 6, 79, 80, 82

PARIS

Biblioteca Nazionale

Lat. 4450: 79

Lat. 4533: 78, 82, 83

RomA

Biblioteca Angelica

275 (già C. 6.1): 149, 150 
Biblioteca Apostolica Vaticana Archivio San Pietro A.29: 5, 60, 72, 84, 93, 94, 95, 96, 97, 98, 99, 100, 102, 195, 106, 107, 109, 110, 112, 113, 115

Barb. Lat. 1410: 73

Chigi E.VIII.245: 5, 60, 73, 94, 95 , 96, 99, 105, 110, 112, 113

Ottoboniano lat. 1726: 86, 122

Pal. lat. 731: 78

Pal. lat. 735: 79

Pal. lat. 742: 78

Pal. lat. 759: 78, 82, 83

Vat. Lat. 1406: 79

Vat. lat. 1428: 79, 82

Vat. Lat. 1739: 79

Vat. lat. 2619: 79

Vat. lat. 5773: 4, 86, 118, 119, 120, 122, 123, 124, 125, 130, 131, 136, $137,138,140,141,142,187$
Biblioteca Nazionale Centrale

Vittorio Emanuele 1511. 84, 113

StUTTGART

Württembergische Landesbibliothek

Jur. fol. 123: 84, 86, 87

TORINO

Biblioteca Nazionale

F.IV.10: 118

F.IV.11: 118

F.IV.13: 118

TÜBINGEN

Universitätsbibliothek

Mc 294: 82

Mc 299: 84, 114 

INDICE DEI PASSI DEL CORPUS IURIS CIVILIS

E DEL CORPUS IURIS CANONICI

Digestum Vetus

1.3.32: 84, 85, 86, 87, 114

2.1: 61

2.1.3: 92

2.1.15: 86

2.3pr: 176

2.3.4: 176

2.4.8.2: 82

2.6.2: 108

2.13.6: 129, 133

2.13.6.9: 129

3.2.13.7: 60,100

4.8.21.10: 81

11.6.1: 98

12.3.3: 91

16.3.24: 80

20.1.3: 94

22.3.10: 138

24.2: 79

24.3.1: 79

28.2.19: 108

28.3.17: 115, 153

31.47.un: 133

Digestum Novum

39.4.16: 92

39.5.26: 168

41.1.55: 109

42.1.15: 81
42.4.7: 81

44.1.3: 98

44.2.1: 94

45.1.132: 169

47.8.4.12: 98

48.19.1: 108

48.19.42: 98,108

48.2.3.4: 101

50.17.92: 129, 133

CODEX

Const. Prooem. II.3: 138

1.1.1: 91

1.3.28: 84

1.4.3.4: 87

1.14.7: 114

1.54.6: 100

2.1: 61,84

2.1.1: 84

2.1.2: 84,176

2.1.3: 84,171

2.1.4: 84

2.1.8: 129

2.3.29: 84

2.27[28].1: 97

2.41.1: 84

2.55.4.1: 138

3.1.13: 81,111

3.9.1: 111 


\begin{tabular}{|c|c|}
\hline 3.13.7: 92 & 6.28.4: 168,170 \\
\hline $3.27 .1: 108$ & 6.30.19: 170 \\
\hline 3.27.2: 108 & 6.30.22.14: 170 \\
\hline 4.10.1: 170 & 6.36.8: 168,170 \\
\hline 4.13: 64 & 6.37.8: 77 \\
\hline 4.18: 139 & $6.47 .1: 170,180$ \\
\hline 4.19: 180 & $6.59[60] .3: 62$ \\
\hline 4.19.2: 180 & 7.33: 86 \\
\hline 4.19.3: 121,180 & 7.47.un: $88,89,90$ \\
\hline 4.19.5: 169 & 7.63: 84,110 \\
\hline 4.19.6: 168,180 & 7.63.2: 111 \\
\hline 4.19.7: 140 & $8.48 .1: 156$ \\
\hline 4.20: 169,180 & $8.52: 23,85,86$ \\
\hline 4.20.20: 180 & $8.53[52] .1: 86$ \\
\hline $4.21: 164,180$ & $8.53[52] .2: 86,87$ \\
\hline 4.21.17: $122,124,125,126,132$ & 9.6.6: 101 \\
\hline 4.21.17.1: 124 & 9.24.2: 60 \\
\hline $4.21 .20: 138,170$ & 12.45: 108 \\
\hline 4.29.22: 169 & \\
\hline 4.30.3: 64,169 & \\
\hline 4.30.13: 64 & INSTITUTIONES \\
\hline $4.31 .4: 170$ & 1.2.9: 86,122 \\
\hline 4.35.22: 169 & \\
\hline 4.37.1: 163 & \\
\hline 4.44.2: 176 & $\begin{array}{l}\text { NOVELLAE } \\
\text { Nov } 44=\text { Coll IV tit } 7 \cdot 130\end{array}$ \\
\hline 4.45.2: 169 & $\begin{array}{l}\text { Nov. } 44=\text { Coll. IV, tit. } 7: 130 \\
\text { Nov } 53=\text { Coll V } 6 \cdot 111\end{array}$ \\
\hline 4.49.4: 169 & Nov. 3 = Coll. 12, tit. $0: 111$ \\
\hline 4.50.6: 121 & \\
\hline 5.51.13: 138 & \\
\hline 6.1.3: 170 & LIBER EXTRA \\
\hline 6.2.3: 170 & 2.19.3: 123 \\
\hline 6.3.10[6]: 62 & $2.19 .7: 123$ \\
\hline $6.7 .2: 60,170$ & $2.19 .11: 123$ \\
\hline 6.11.2: 170 & 2.22.10: 91 \\
\hline 6.14.3: 170 & 2.22.2: 123 \\
\hline 6.18.1: 170 & $2.22 .6: 123$ \\
\hline $6.20 .13: 168,170$ & 2.22.9: 123 \\
\hline 6.20.17: 151 & 2.22.15: 123 \\
\hline 6.22.8: 168,169 & $2.32 .15: 134$ \\
\hline 6.23.7: 96, 180 & \\
\hline 6.23.24: 106 & \\
\hline $6.23 .27: 180$ & LIBER SEXTUS \\
\hline $6.24 .1: 104$ & 5.5: 118 \\
\hline 6.26.8: 168 & 5.5.2: 116 \\
\hline
\end{tabular}


BiBLIOGRAFIA

Abbreviazioni e sigle:

ACaMo = Archivio Capitolare di Modena.

AIMAe = L.A. Muratori, Antiquitates Italicae Medii Aevi, 6 voll., Mediolani, ex typographia Societatis Palatinae in regia curia, 1738-1742.

AMASLA $=$ «Atti e Memorie dell'Accademia Nazionale di Scienze Lettere e Arti di Modena».

AMDSPMo $=$ «Atti e Memorie della Deputazione di Storia Patria per le Antiche Provincie Modenesi».

ASCMo = Archivio Storico Comunale di Modena .

ASMo $=$ Archivio di Stato di Modena.

Autographa, I.1. = G. Murano (a cura di), Autographa, I.1. Giuristi, giudici e notai (sec. XII-XV med.), Bologna 2012.

Autographa, I.2. = G. Murano (a cura di), Autographa, I.2. Giuristi, giudici e notai (sec. XII-XV), Imola (Bo) 2016.

DBI = Dizionario Biografico degli Italiani.

DBGI = Dizionario biografico dei giuristi italiani (XII-XX secolo), a cura di I. Birocchi, E. Cortese, A. Mattone, M.N. Miletti, 2 voll., Bologna 2013.

$\mathrm{ED}=$ Enciclopedia del Diritto.

IC $=$ «Ius Commune».

IMU $=$ «Italia Medioevale e Umanistica».

QSUP $=$ «Quaderni per la Storia dell’Università di Padova».

RIDC $=$ «Rivista Internazionale di Diritto Comune».

RIS = Rerum Italicarum Scriptores. 
RM = E.P. VICINI (a cura di), Respublica Mutinensis (1306-1307), 2 voll., Milano 1929-32 (Corpus Statutorum Italicorum, 11-14).

RPCM = L. Simeoni, E.P. Vicini (a cura di), Registrum Privilegiorum Comunis Mutinae, I, Reggio E. 1940; II, Modena 1949.

RSDI $=$ «Rivista di Storia del Diritto Italiano».

$\mathrm{SM}=\ll$ Studi Medievali $»$.

$\mathrm{TR}=$ «Tijdschrift voor Rechtsgeschiedenis».

TUI = Tractatus Universi Iuris.

Fonti e letteratura:

Abbondanza R., voce Arsendi Raniero, in DBI, 4, Roma 1962, pp. 330339.

Alberico da Rosciate, Commentarium de statutis, in TUI, II, Venetiis 1584, ff. 2r-85v.

Alberico da Rosciate, Commentaria, 8 voll., Venetiis [Società dell'aquila che si rinnova] 1585 (rist. an. Bologna 1974-79).

Alberico da Rosciate, Dictionarium iuris tam civilis quam canonici, Venetiis, Impensis Societatis Librorum Legalium, 1603.

Albini G. (a cura di), Le scritture del Comune. Amministrazione e memoria nelle città dei secoli XII e XIII, Torino 1998.

Al KalaK M., Il monastero di Sant'Eufemia. Origini, storia e sviluppo di una religiosità al femminile, in AMASLA, s. VIII, VIII/1 (2005), pp. 253-350.

Amelotti M., Notaio (diritto romano), in ED, 28, Milano 1978, pp. 553-559.

Andreolli B., Dai 'Figli di Manfredo' ai Pio: genesi di una signoria, in P. Bonacini, A.M. Ori (a cura di), Storia di Carpi, I. La città e il territorio dalle origini all'affermazione dei Pio, Carpi (Mo) 2008, pp. 395-412.

ANDReolli B., La soccida nel territorio mirandolese del tardo medioevo, in AMDSPMo, s. XI, VII (1984), pp. 85-99 (riedito in ID., Signori e contadini nelle terre dei Pico, Modena 1988, pp. 61-78).

Andreolli B., Gatti D., Greci R. et alii (a cura di), Repertorio della cronachistica emiliano-romagnola (secc. IX-XV), Roma 1991.

Andrich G. (a cura di), Glosse di Antonio Porcellino ai nomi di alcuni giureconsulti iscritti nel s. Collegio de' giuristi di Padova, da un manoscritto dell'archivio universitario, Padova 1892.

Angeli Ubaldi, In I. atque II. Digesti Veteris partem Commentaria [...], Venetiis, Ad signum Aquilae se renovantis, 1580. 
Angelo da Chivasso, Summa angelica reverendi patris fratris Angeli del Clavasio [...], Lugduni, apud Scipionem de Gabiano in Vico mercuriali sub signo fontis, 1534 .

Annales Patavini, a cura di A. Bonardi, in RIS, II ed., VIII/1, Città di Castello 1903, pp. 177-265.

Annales Veteres Mutinensium ab anno MCXXXI usque ad anno MCCCXXXVI, in RIS, I ed., XI, Mediolani, 1728, coll. 53-86.

Antognoli G., Conservator pacis et iustitiae. La signoria di Taddeo Pepoli a Bologna (1337-1347), Bologna 2004.

Antonazzi G., Lorenzo Valla e la polemica sulla donazione di Costantino con testi inediti dei secoli XV-XVII, Roma 1985.

Antonius Matharellus, Ad Franc. Hotomani Franco-Galliam Antonii Matharelli responsio, in qua agitur de initio regni Franciae, successione regum, publicis negotiis et politia, ex fide annalium nostrorum Germaniaeque et aliarum gentium graecisque et latinis scriptoribus, Lutetiae, ex officina F. Morelli, 1575.

Arabeyre P., voce Jean (Ignaeus) Feu, in Dictionnaire historique des juristes français, $X I I^{e}-X X^{e}$ siècle, sous la direction de P. Arabeyre, J.-L. Halpérin, J. Krynen, Paris 2007, p. 329.

Ascheri M., Saggi sul Diplovatazio, Milano 1971.

Ascheri M., Tribunali, giuristi e istituzioni dal medioevo all'età moderna, Bologna 1989.

Ascheri M., Bartolo da Sassoferrato: introduzione a un giurista globale, in Mundos medievales. Espacios, sociedades y poder. Homenaje al Profesor José Angel Garcìa de Cortazar y Ruiz de Aguirre, editores B. Arizaga Bolumburu, D. Mariño Velras, C. Diez Herrera et alii, II, Santander 2012, pp. 1029-1040.

Ascheri M., Bartolo da Sassoferrato: il civilista 'globale' del Medioevo, in E. Mecacci, M.A. Panzanelli Fratoni (a cura di), Bartolo da Sassoferrato a Siena nel VII centenario della nascita. Manoscritti, incunaboli, cinquecentine. Con un ricordo di Domenico Maffei, Siena 2014, pp. 13-22.

Ascheri M., Diplovatazio (Diplovataccio) Tommaso, in DBGI, II, pp. 772773.

Ascheri M., G.B. Caccialupi (1420 ca.-1496) fautore dei Monti di Pietà, in Grundlagen des Rechts. Festschrift für Peter Landau zum 65. Geburtstag, hrsg. von R.H. Helmholz, P. Mikat, J. Müller und M. Stolleis, Paderborn-München-Wien-Zürich 2000, pp. 643-653.

Ascheri M., La normativa di diritto comune per lo straniero nell'opera di 
G.B. Caccialupi, in Stranieri e forestieri nella Marca dei secc. XIVXVI. Atti del XXX Convegno di studi maceratesi. Macerata, 19-20 novembre 1994, Macerata 1996, pp. 93-113.

Astegiano L., Codex Diplomaticus Cremonae, 715-1334, 2 voll., Augustae Taurinorum 1895-98 (rist. an. Sala Bolognese 1983).

Atti \& formule di Rolandino. XXXVIII Congresso Nazionale del Notariato, Bologna, 8-11 ottobre 2000, Bologna 2000.

Avesani R., Il preumanesimo veronese, in Storia della cultura veneta, II. Il Trecento, Vicenza 1976, pp. 111-141.

Azonis, Lectura Azonis et magni apparatus ad singulas leges duodecim librorum Codicis Iustiniani, Parisiis, Apud Sebastianum Nivellium sub Ciconiis, via Iacobaea, 1581.

Azonis, Summa super codicem, in Corpus glossatorum juris civilis, II, Augustae Taurinorum 1966, pp. 1-346.

Azonis Iurisconsultissimi in ius civile Summa, Lugduni 1564.

Baietto L., Scrittura e politica. Il sistema documentario dei comuni piemontesi nella prima metà del secolo XIII, in «Bollettino Storico-Bibliografico Subalpino», XCVIII/I (2000), pp. 105-165; XCVIII/II (2000), pp. 473-528.

Balbi G., Catholicon, Mainz 1460 (rist. an. Westmead 1971).

Baldi Ubaldi, Consiliorum sive responsorum liber tertium, Venetiis, Francesco de' Franceschi, Gaspare Bindoni, eredi di Niccolò Bevilacqua, Damiano Zenaro, 1575.

Baldi lectura feudorum, Venetiis, apud Iuntas, 1580.

BALdi Ubaldi, In primum, secundum, tertium codicis libros commentaria [...], Venetiis, apud Iuntas, 1586.

BALDI UBALDI, In quartum et quintum codicis libros commentaria [...], Venetiis, apud Iuntas, 1599.

Baldi Ubaldi, In sextum codicis librum commentaria [...], Venetiis, apud Iuntas, 1599.

Baldi Ubaldi, In VII, VIII, IX, X et XI Codicis libros Commentaria [...], Venetiis, apud Iuntas, 1615.

Baldi Ubaldi, Tractatus solemnis de constituto, in De mercatura decisiones et tractatus varii et de rebus ad eam pertinentibus, Lugduni, expensis Petri Landry, 1608, pp. 629a-632b. (altre edd. Lione 1582, 1590, 1592, 1593, 1610, 1621; Francoforte 1612, 1652; Colonia 1622, 1630; anche in TUI, VI/I, ff. 38r-39r).

Baracchi O.-Manicardi A., Modena: quando c'erano i canali, Modena 1985. 
Baracchi O.-Giovannini C., Il Duomo e la torre di Modena. Nuovi documenti e ricerche, Modena 1988.

Bartoli de Saxoferrato, Prima super Digesto Veteri [...], Lugduni, ex officina Sebastiani Gryphij, 1533.

Bartoli de Saxoferrato, In primam Infortiati partem, in ID., Omnium Iuris interpretum antesignani commentaria [...], t. III, Venetiis, apud Iuntas, 1596.

Bartholus de Saxoferrato, Commentaria cum additionibus Thomae Diplovatatii [...], curavit G. Polara, IX, Roma 1996 (rist. an. dell'ed. curata e pubblicata dal Diplovatazio nel 1520).

Bartocci A., Giovanni d'Andrea (Johannes Andreae de Bononia), in DBGI, I, pp. 1008-1012.

Bassanellus. Mar. Mantui, Bonauiti, Patauini, iuriscon. Caesareique comitis minimi, Colloquia, seu dialogi CC iuris, nunc primum in literatorum gratiam editi [...],Venetiis, ex officina Erasmiana Vincentij Valgrisij, 1553.

BASSANi A., Note a margine della vita e delle opere di Nello Cetti da San Gimignano, in M.G. DI ReNZo VILlATA (a cura di), Lavorando al cantiere del 'Dizionario Biografico dei Giuristi Italiani (XII-XX sec.)', Milano 2013, pp. 429-463.

BAUMGäRTNER I., Martinus Garatus Laudensis. Ein italienischer Rechtsgelehrter des 15. Jahrhunderts, Köln-Wien 1986.

BAUMGÄRTNER I., «De privilegiis doctorum». Über Gelehrtenstad und Doktorwürde im späten Mittelalter, in «Historisches Jahrbuch», 106 (1986), pp. 298-332.

BAUMGÄRTNER I., An expediat habere multos libros. Zum Wert von Büchern oder der Bildungshorizont eines Oldradus de Ponte, in «Archiv für Kulturgeschichte», 74 (1992), pp. 303-322.

BAUMgäRTnER I., Quidam presbiter beneficialis. Der niedere Klerus in den Rechtsgutachten des späten Mittelalters, in «Zeitschrift der Savigny-Stiftung für Rechtsgeschichte. Kanonistiche Abteilung», LXXXI (1995), pp. 189-224.

Bedoni G., G.B. Scanaroli e L.A. Muratori, in AMDSPMo, s. VIII, XI (1959), pp. 222-235.

Bedoni G., La medicina a Modena durante il medioevo regolata dal diritto canonico e comunale, in AMDSPMo, s. XI, XVIII (1996), pp. 73-96.

Bedoni G., Niccolò Mattarelli: il politico, il docente, il giurista, in AMDSPMo, s. XI, V (1983), pp. 75-106. 
Bellomo M., Due 'libri magni quaestionum disputatarum' e le 'quaestiones' di Riccardo da Saliceto, in ID., Inediti della giurisprudenza medievale, Frankfurt am M. 2011, pp. 245-277 (già in «Studi Senesi», s. III, LXXXI, 1969, pp. 256-291).

Bellomo M., Glossae contrariae di Cino da Pistoia, in TR, 38 (1970), pp. 433-447.

Bellomo M., Legere, repetere, disputare. Introduzione ad una ricerca sulle «quaestiones» civilistiche, in ID., Aspetti dell'insegnamento giuridico nelle università medievali, I. Le «Quaestiones disputatae», Reggio Calabria 1974, pp. 13-81.

Bellomo M., Le istituzioni particolari e i problemi del potere. Dibattiti scolastici dei secoli XIII-XIV, in ID., Inediti della giurisprudenza medievale, Frankfurt am M. 2011, pp. 207-244 (già, con modifiche, in Studi in memoria di Giuliana D’Amelio, I. Studi storico-giuridici, Milano 1978, pp. 1-40).

Bellomo M., Società e istituzioni in Italia dal Medioevo agli inizi dell'età moderna, Catania 1982.

Bellomo M., Giuristi cremonesi e scuole padovane. Ricerche su Nicola da Cremona, in ID., Inediti della giurisprudenza medievale, Frankfurt am M. 2011, pp. 137-172 (già in Studi in onore di Ugo Gualazzini, I, Milano 1981, pp. 81-112).

Bellomo M., Consulenze professionali e dottrine di professori. Un inedito «consilium domini Accursii», in Studi in onore di Cesare Sanfilippo, III, Milano 1983, pp. 17-36 (già in «Quaderni catanesi di studi classici e medievali», 7 (1982), pp. 199-219).

Bellomo M., Factum proponitur certum, sed dubium est in iure, in Die Kunst der Disputation. Probleme der Rechtsauslegung und Rechtswendung im 13. und 14. Jahrhundert, hrsg. von M. Bellomo, München 1997, pp. 1-28.

Bellomo M., Tracce di lectura per viam quaestionum in un manoscritto del Codex conservato a Rovigo, in RIDC, 8 (1997), pp. 217-273.

Bellomo M., Intorno a Roffredo Beneventano: professore a Roma?, in M. Bellomo (a cura di), Scuole, diritto e società nel Mezzogiorno medievale d'Italia, I, Catania 1985, pp. 135-181 (anche in ID., Medioevo edito e inedito, III. Profili di giuristi, Roma 1998 (I libri di Erice, 20), pp. 5-54).

Bellomo M., L'Europa del diritto comune, Roma 1994.

Bellomo M., La scienza del diritto al tempo di Federico II, in RIDC, 3 (1992), pp. 173-196 (poi in P. Toubert, A. Paravicini Bagliani (a cura di), Federico II e le scienze, Palermo 1994 pp. 86-106). 
Bellomo M., 'Factum' e 'ius'. Itinerari di ricerca tra le certezze e i dubbi del pensiero giuridico medievale, in ID., Medioevo edito e inedito, II. Scienza del diritto e società medievale, Roma 1997, pp. 63-89 (già in RIDC, 7, 1996, pp. 21-46).

Bellomo M., Saggio sulle distinctiones di Giovanni Calderini, in ID., Inediti della giurisprudenza medievale, Frankfurt am Main 2001, pp. 355384 (già in RIDC, 9, 1998, pp. 165-196).

Bellomo M., Saggio sull'Università nell'età del Diritto Comune, Roma 1996.

Bellomo M., I fatti e il diritto tra le certezze e i dubbi dei giuristi medievali (secoli XIII-XIV), Roma 2000.

Bellomo M., Quaestiones in iure civili disputatae. Didattica e prassi colta nel sistema del diritto comune fra Duecento e Trecento. Contributi codicologici di Livia Martinoli, in Appendice, Roma 2008 (Fonti per la storia dell'Italia medievale. Antiquitates 31).

Bellomo M., Società e diritto nell'Italia medievale e moderna, Roma 2002.

Bellomo M., Sulle tracce d'uso dei «libri legales», in ID., Medioevo edito $e$ inedito, I, Roma 1997, pp. 121-138 (già in Civiltà Comunale: Libro, Scrittura, Documento. Atti del Convegno, Genova, 8-11 novembre 1988, Genova 1989, pp. 33-51).

Bellomo M., Giuristi bolognesi del tempo di Taddeo Pepoli. Maccagnano e Tommaso degli Azzoguidi, in ID., Inediti della giurisprudenza medievale, Frankfurt am Main 2011, pp. 301-345 (già con modifiche in ID., Aspetti dell'insegnamento giuridico nelle Università medievali. I. Le 'quaestiones disputatae', Reggio Calabria 1974, pp. 119-194).

Belloni A., Professori giuristi a Padova nel secolo XV. Profili bio-bibliografici e cattedre, Frankfurt am Main 1986.

Belloni A., Bartolo studente e maestro e i suoi commentari, in Bartolo da Sassoferrato nel VII centenario della nascita: diritto, politica, società. Atti del L Convegno storico internazionale, Todi-Perugia, 13-16 ottobre 2013, Spleto 2014, pp. 559-584.

Benatti C., voce Guido da Suzzara, in DBGI, I, pp. 1093-1094.

Bernardini Scodobil, In auctenticam habita C. Ne filius pro patre, omnibus iuris utriusque studiosis iuvenibus frugifera comprimis ac festivissima interpretatio, Venetiis, apud Cominum de Tridino Montisferrati, 1547 (anche Venetijs, apud Cominum de Tridino Montisferrati, 1548).

Bernicoli S., Governi di Ravenna e di Romagna dalla fine del secolo XII alla fine del secolo XIX, Ravenna 1898 (rist. an. Sala Bolognese 1968).

Bertazzoli B., Consiliorum seu responsorum iuris in criminalibus et pena- 
libus controversiis emissorum liber primus. Multiplici rerum cognitione ad quotidianam criminum praxim spectantium maxime refertus a Claudio Bertazzolio, auctoris filio, Venetiis, apud Ioannem Baptistam Somaschum, 1584.

Bertoni G. (a cura di), Atlante storico-paleografico del Duomo di Modena, Modena 1909.

Bertoni G.-VIcIn E.P., I codici di un medico modenese del secolo XIV (Jacopino Cagnoli), Modena 1904 (estratto da AMDSPMo, s. V, IV, 1904).

Bertram M., Maffei P., A Catalogue of the Manuscripts in the Chapter Library of La Seu d'Urgell, in Iuris Historia. Liber Amicorum Gero Dolezalek, ed. by V. Colli and E. Conte, Berkeley 2008, pp. 61-72.

BESTA E., L'opera d'Irnerio (contributo alla storia del diritto italiano), II. Glosse inedite d'Irnerio al Digestum vetus, Torino 1896.

Besta E., Su due opere sconosciute di G. e di Arsendino Arsendi, Venezia 1896.

Besta E., Baldo e la storia letteraria del diritto, in L'opera di Baldo per cura dell'Università di Perugia nel V centenario della morte del grande giureconsulto, Perugia 1901, pp. 81-111.

BESTA E., Per la storia della nostra letteratura processuale nella prima metà del secolo decimo terzo, in Studi di diritto romano, di diritto moderno e di storia del diritto pubblicati in onore di V. Scialoja nel XXV anniversario del suo insegnamento, II, Milano 1905, pp. 655-670.

Besta E., Fonti: legislazione e scienza giuridica dalla caduta dell'impero romano al secolo decimosesto, Milano 1925 (Storia del diritto italiano, pubblicata sotto la direzione di P. Del Giudice, vol. I, parte II).

Besta E., Riccardo Malombra professore nello studio di Padova consultore di stato in Venezia, Venezia 1894.

BetTetini A., voce Pascipovero (Passipoverus), in DBGI II, pp. 1516-1517.

Bevilacoua M., Nicolai de Mactarellis Consilium de pace promissa et non servata, in «Latinitas», VIII (1960), pp. 218-220.

Bevilacoua M., Una «quaestio» di Niccolò Mattarelli (Vat. Lat. 10726), in Collectanea Vaticana in honorem Anselmi M. card. Albareda a Bibliotheca Apostolica edita, I, Città del Vaticano 1962, pp. 139-157.

Bezemer C.H., Les répétitions de Jacques de Révigny. Recherches sur la répétition comme forme d'enseignement juridique et comme genre littéraire, suivies d'un inventaire des textes, Leiden 1987.

Billanovich G., Epitafio, libri e amici di Alberico da Rosciate, in IMU, 3 (1960), pp. 251-261 (riedito con integrazioni con il titolo Cultura bergamasca del Trecento, in M. CORTESI (a cura di), Statuti rurali e statuti 
di valle. La provincia di Bergamo nei secoli XIII-XVIII. Atti del convegno, Bergamo 5 marzo 1983 (Fonti per lo studio del territorio bergamasco, V), Bergamo 1984, pp. 21-41).

Billanovich G., Il preumanesimo padovano, in Storia della cultura veneta, II. Il Trecento, Vicenza 1976, pp. 19-110.

Billanovich G., I primi umanisti padovani e gli epitafi di Seneca e di Livio, in IMU, 43 (2002), pp. 115-146.

Birocchi I.-Mattone A., voce Can (Cane, a Canibus, de Canibus) Giovanni Giacomo, in DBGI, I, pp. 407-408.

BIROcCHI I., voce Ranieri (Rainerio) da Perugia, in DBGI, II, pp. 1654-1655.

BIRocchI I., voce Rolandino Passeggeri (Passaggeri), in DBGI, II, pp. 1717 1720.

BiRocchI I., voce Salatiele, in DBGI, II, pp. 1769-1771.

BiRocchI I., voce Zaccaria di Martino, in DBGI, II, pp. 2074-2075.

Boccolari G., Lodovico Vedriani e la storia di Modena, in AMDSPMo, s. X, VII (1972), pp. 51-63.

Bonacin P., Dinamiche istituzionali e circolazione dei podestà a Modena nel secolo XIII, in AMASLA, s. VIII, IV (2000-2001), pp. 411-484.

Bonacin P., Il «Registrum Comunis Mutine» (1299). Politica e amministrazione corrente del Comune di Modena alla fine del XIII secolo, Modena 2002 [Quaderni dell'Archivio Storico, XV].

Bonacin P. (a cura di), La Fabbrica di San Geminiano. Regesto del Codice Capitolare O.II.11. Regesto di J. Elefante. Presentazione di F. Piccinini, Modena 2012.

Bonacin P., Alle origini del codice O.II.11. Il Liber iurium della Fabbrica del Duomo, in P. Bonacin (a cura di), La Fabbrica di San Geminiano, pp. 9-44.

Bonacini P., Il governo del Comune cittadino tra Due e Trecento, in P. Bonacini, A.M. Ori (a cura di), Storia di Carpi, I. La città e il territorio dalle origini all'affermazione dei Pio, Carpi (Mo) 2008, pp. 353-394.

Bonacini P., Scribarum turba Mutine. Notai a Modena nel tardo medioevo tra cultura, politica e società, in G. TAMBA, E. TAVILLA (a cura di), Nella città e per la città. I notai a Modena dal IX al XX secolo, Milano 2013, pp. 47-69.

Bonato E. (a cura di), Il «Liber contractuum» dei Frati Minori di Padova e di Vicenza (1263-1302), Roma 2002.

BordinI S., Per un profilo di Uberto da Bobbio. Ricerche e ipotesi di lavoro su un giurista del primo Duecento, in N. Sarti-S. Bordini, L'avvocato 
medievale tra mestiere e scienza giuridica. Il Liber cautele et doctrine di Uberto da Bobbio (...1211-1245), Bologna 2011, pp. 9-98.

Borino I.B. (a cura di), Codices Vaticani Latini. Codices 10701-10875 (Bibliothecae Apostolicae Vaticanae codices manu scripti recensiti, 9,1), in Bibliotheca Vaticana 1947.

Bortolami S., Politica e cultura nell'import-export del personale itinerante di governo dell'Italia medioevale: il caso di Padova comunale, in J.-C. Maire Vigueur (a cura di), I podestà dell'Italia comunale, I. Reclutamento e circolazione degli ufficiali forestieri (fine XII sec.-metà XIV sec.), Roma 2000, pp. 203-258.

Boscaino M., voce Facciolati Iacopo, in DBI, 44, Roma 1994, pp. 65-68.

Braidi V., I Modenesi nel Trecento. Il «Liber magne masse populi civitatis Mutine», Modena 2004.

Braidi V., Lo Statuto del Collegio dei notai dell'anno 1336, in G. TAmba, E. TAvilla (a cura di), Nella città e per la città. I notai a Modena dal IX al XX secolo, Milano 2013, pp. 5-18.

Braidi V., Tavilla E. (a cura di), Statuti del Collegio dei giudici e degli avvocati della città di Modena 1270-1337, Modena 2006.

BRIANI G., Aggiunta a' Ragguagli di Parnaso del molt'Illustre et Eccellentissimo Signor Trajano Boccalini romano, in Amsterdam, appresso Giovanni Blaeu, 1669.

BrinKhus G., Mentzel-Reuters A., Handschriftenkatalog der Universitätsbibliothek Tübingen, 1. Die lateinischen Handschriften, teil 2. Signaturen Mc 151 bis Mc 379 sowie die lateinischen Handschriften bis 1600 aus den Signaturgruppen Mh, Mk und aus dem Druckschriftenbestand, Wiesbaden 2001.

Buigues O.G., La solucion amistosa de los conflictos, en derecho romano: el arbiter ex compromisso, Madrid 1990.

Caccialupi G.B., Tractatus de modo studendi, \& vita doctorum [...], Papie, per Iacob de Burgofranco, 1510.

Caillemer R., Les débuts de la science du droit en Provence: Iohannes Blancus Massiliensis, in Congrès des sociétés savantes de Provence, Aix-en-Provence - Marseille 1907, pp. 767-792.

Calasso F., Medio Evo del diritto. I. Le Fonti, Milano 1964.

Cammarosano P., Prospettive di ricerca dal Liber Censuum del Comune di Pistoia, in E. VAnnucchi (a cura di), Pistoia e la Toscana nel Medioevo. Studi per Natale Rauty, Pistoia 1997 (Biblioteca Storica Pistoiese, I), pp. 61-69. 
CAmpori C., Intimazioni legali del vescovo Ardizzone de' Conti al Comune di Modena per la correzione degli statuti del 1283, in «Atti e Memorie delle RR. Deputazioni di Storia Patria per le Provincie Modenesi e Parmensi», I (1863), pp. 337-340.

CAMPori C., Del governo a Comune in Modena secondo gli Statuti ed altri documenti sincroni, in Statuta civitatis Mutine, Parmae 1864, pp. IXCCLXXX (Monumenti di Storia Patria delle Provincie modenesi. Serie degli Statuti, tomo I).

Cappelli A., Dizionario di abbreviature latine ed italiane, Milano $1912^{2}$.

CAPucci M., Lodovico Vedriani e il biografismo artistico municipale, in A. Prosperi (a cura di), Il piacere del testo. Saggi e studi per Albano Biondi, Roma 2001, pp. 555-571.

CASElli G.C. (a cura di), Corpus glossatorum juris civilis, V.3, Augustae Taurinorum 1975 (rist. an. dell'ed. Lugduni, per Io. de Cambrey, 1522).

Catálogo de los manuscritos jurícos de la biblioteca capitular de la Seu d'Urgell, elaborado bajo la direccíon de Antonio García y García por M. Bertram, G. Fransen, A. Antonio García y García, D. Maffei, P. Maffei, B. Marqués Sala, A. Pérez Martín, La Seu d'Urgell 2009.

Cavalca D., Il bando nella prassi e nella dottrina giuridica medievale, Milano 1978.

Cavallar O., Degenring S., Kirshner J., A grammar of signs. Bartolo da Sassoferrato's Tract on insignia and Coats of Arms, Berkeley 1994.

Cecina A., Notizie istoriche della città di Volterra alle quali si aggiunge la serie de' podestà, e capitani del popolo di essa, In Pisa, per Gio. Paolo Giovannelli, e compagni stampat. dell'Almo Studio Pisano, 1758.

Cerasi L., voce Andrea Gloria, in DBI, 57, Roma 2001, pp. 411-415.

Cerroni M., voce Guglielmo da Pastrengo, in DBI, 61 (2004), pp. 17-22.

Cesarini Sforza W., Sull'ufficio bolognese dei Memoriali (sec. XIII-XV), in «L'Archiginnasio», IX (1914), pp. 379-392.

Chiappini L., Gli Estensi. Mille anni di storia, Ferrara 2001.

Cino da Pistoia, Lectura super Codice, a cura di G. Polara, 2 voll., Roma 1998 (rist. an. dell'ed. Francofurti ad Moenum, Impensis Sigismundi Feyerabendt, 1578).

Cipolla C., Codici bobbiesi della Biblioteca Nazionale Universitaria di Torino, I, Milano 1907.

Cipriani R., Codici miniati dell'Ambrosiana. Contributo a un catalogo, Milano 1968.

Cochetti M., Repertori bibliografici del Cinquecento, Roma 1987.

CoING H. (a cura di), Handbuch der Quellen und Literaturn der neueren 
europäischen Privatrechtsgeschichte, I. Mittelalter (1100-1500). Die gelehrten Rechte und die Gesetzgebung, München 1973.

Colle F.M., Storia scientifico-letteraria dello Studio di Padova, II, Padova 1824.

Colli G., Introduzione bibliografica, in Tractatus Universi Iuris extravagantes, I. Tractatus de dignitate et potestate seculari, Venetiis 1548, Napoli 2005, pp. XIII-XVI

Colli G., Le edizioni dell'Index librorum omnium iuris civilis et pontificii di Giovanni Battista Ziletti. Sulle tracce dei libri giuridici proibiti nella seconda metà del XVI sec., in M. Ascheri, G. Colli (a cura di), Manoscritti, editoria, biblioteche dal medioevo all'età contemporanea. Studi offerti a Domenico Maffei per il suo ottantesimo compleanno, I, Roma 2006, pp. 205-244.

Colli G., Per una bibliografia dei trattati giuridici pubblicati nel XVI secolo, I. Indici dei Tractatus universi iuris, Milano 1994 (Ius Nostrum, 20).

Colli G., Per una bibliografia dei trattati giuridici pubblicati nel XVI secolo, I. Bibliografia delle raccolte. Indici dei trattati non compresi nei Tractatus universi iuris, Roma 2004 (ius Nostrum, 28).

Colli V., Il cod. 351 della Biblioteca capitolare «Feliniana» di Lucca: editori quattrocenteschi e libri consiliorum di Baldo degli Ubaldi (13271400), in M. Ascheri (a cura di), Scritti di storia del diritto offerti dagli allievi a Domenico Maffei, Padova 1991, pp. 255-282.

Colli V., Le opere di Baldo. Dal codice d'autore all'edizione a stampa, in C. Frova, M.G. Nico Ottaviani, S. Zucchini (a cura di), VI Centenario della morte di Baldo degli Ubaldi 1400-2000, Perugia 2005, pp. 25-85.

Colli V., Lo Speculum iudiciale di Guillaume Durand: codice d'autore ed edizione universitaria, in Juristische Buchproduktion im Mittelalter, hrsg. von V. Colli, Frankfurt am Main 2002, pp. 517-566.

Colli V., La biblioteca di Bartolo. Intorno ad autografi e copie d'autore, in Bartolo da Sassoferrato nel VII centenario della nascita: diritto, politica, società. Atti del L Convegno storico internazionale, Todi-Perugia, 13-16 ottobre 2013, Spoleto 2014, pp. 67-105.

Condorelli O., Sul contributo dei canonisti alla definizione del concetto di interesse. Frammenti di ricerca (metà sec. XII-metà sec. XIII), in Der Einfluß der Kanonistik auf die europäische Rechtskultur, V. Das Recht der Wirtschaft, hrsg. von D. von Mayenburg, O. Condorelli, F. Roumy, M. Schmoeckel, Köln-Weimar-Wien 2016, pp. 23-60.

Conetti M., La dottrina dell'impero e la donazione di Costantino in Alberico da Rosciate, in Studi di storia del diritto, II, Milano 1999, pp. 303-405. 
Conte E., Un sermo pro petendis insigniis al tempo di Azzone e Bagarotto, in RSDI, LX (1987), pp. 71-86.

Coppr E., Le università italiane nel medio evo, Firenze $1880^{2}$ (rist. an. Bologna 1978).

Corbino A., Il danno qualificato e la lex Aquilia. Corso di diritto romano, Padova $2008^{2}$.

Cortese E., La norma giuridica. Spunti teorici nel diritto comune classico, 2 voll., Milano 1962-64.

Cortese E., Scienza di giudici e scienza di professori tra XII e XIII secolo, in Legge, giudici, giuristi. Atti del convegno tenuto a Cagliari nei giorni 18-21 maggio 1981, Milano 1982, pp. 93-148.

Cortese E., Alle origini della scuola di Bologna, in RIDC, 4 (1993), pp. 7-49.

Cortese E., Il diritto nella storia medievale, II. Il basso medioevo, Roma 1995.

Cortese E., Il Rinascimento giuridico medievale, Roma $1996^{2}$ (rielaborazione di ID., Legisti, canonisti e feudisti: la formazione di un ceto medievale, in Università e società nei secoli XII-XVI. Atti del IX convegno internazione, Pistoia, 20-25 settembre 1979, Pistoia 1982, pp. 195-281).

Cortese E., Controversie medievali sul dominio utile: Bartolo e il quidam doctor de Aurelianis, A. Padoa Schioppa, G. Di Renzo Villata, G.P. Massetto (a cura di), in Amicitiae pignus. Studi in ricordo di Adriano Cavanna, I, Milano 2003, pp. 613-635.

Cortese E., Jean Feu a Pavia nel 1509-1510. Propaganda francese nella Lombardia conquistata, in "Ins Wasser geworfen und Ozeane durchquert». Festschrift für Knut Wolfgang Nörr, hrsg. von M. Ascheri, F. Ebel, M. Heckel et alii, Köln-Weimar-Wien 2003, pp. 121-143.

Cortese E., Intorno all'edizione di Bartolo curata dal Diplovatazio e alla sua ristampa anastatica, in Iuris Historia. Liber Amicorum Gero Dolezalek, ed. by V. Colli and E. Conte, Berkeley 2008, pp. 369-385.

CORTESE E., voce Baldo degli Ubaldi, in DBGI, I, pp. 149-152.

Cortese E., voce Canetoli (Canedoli, de Canitulo, Canedulo) Giovanni, in DBGI, I, p. 410.

Cortese E., voce Paolo di (da) Castro, in DBGI, II, pp. 1505-1507.

Cortese E., voce Pepo, in DBGI, II, pp. 1532-1533.

Cortese E., voce Pillio da Medicina, in DBGI, II, pp. 1587-1590.

Coser E., Giansante M., Le locazioni di bestiame nel bolognese fra XIII e XIV secolo da un registro di conti della famiglia Guastavillani, in «At- 
ti e Memorie della Deputazione di Storia Patria per le Province di Romagna», 53 (2002), pp. 223-242.

Coser E., Giansante M. (a cura di), Libro di conti della famiglia Guastavillani (1289-1304), Bologna 2003.

Cremaschi G., Contributo alla biografia di Alberico da Rosciate, in «Bergomum», 30/1 (1956), pp. 3-102.

Crescenzi V., La rappresentazione dell'evento giuridico. Origini e struttura della funzione documentaria, Roma 2005.

Crescenzi V., L'ordine isonomico e il problema della struttura della prova: la prova per documenti, in F. Cerrone e G. Repetto (a cura di), Alessandro Giuliani: l'esperienza giuridica fra logica ed etica, Milano 2012 (Per la storia del pensiero giuridico moderno, 95), pp. 393-430.

Cronaca modenese di Tommasino de' Bianchi detto de' Lancellotti, XI, Modena 1881 [Monumenti di Storia Patria delle Provincie Modesesi. Serie delle Cronache, tomo XII].

Cursi M.F., Iniuria cum damno. Antigiuridicità e colpevolezza nella storia del danno aquiliano, Milano 2002.

Danusso C., Ricerche sulla «Lectura feudorum» di Baldi degli Ubaldi, Milano 1991.

D’Amelio G., voce Caccialupi Giovanni Battista, in DBI, 15, Roma 1972, pp. $790-797$.

D’Amelio G., Il Dictionarium iuris di Jacques de Revigny, in TR, 40 (1972), pp. 43-72.

De Caro G., voce Briani Girolamo, in DBI, 14, Roma 1972, pp. 211-213.

Del Negro P., Da Iacopo Facciolati a Francesco Maria Colle la continuazione dei Fasti Gymnasii Patavini dal 1760 al 1763, in QSUP, 32 (1999), pp. 197-212.

Del Negro P. (a cura di), Clariores. Dizionario biografico dei docenti e degli studenti dell'Università di Padova, Padova 2015.

Del Re N., Farinacci giureconsulto romano (1544-1618), in «Archivio della Società Romana di Storia Patria», LXXXXVIII (1975), pp. 135-220.

Della Fontana G., Il collegio degli avvocati di Modena (1337-1797), in AMDSPMo, s. VI, II (1960), pp. 58-70.

Desanti L., La legge Aquilia. Tra verba legis e interpretazione giurisprudenziale, Torino 2015.

Di CesARE F. (a cura di), Catalogo dei manoscritti in scrittura latina datati per indicazione di anno, di luogo o di copista. II. Biblioteca Angelica di Roma, Torino 1982. 
Di Noto Marrella S., La nobilitazione del professore dopo vent'anni di insegnamento: applicazioni di C. 12.15 in età moderna, in Nozione, formazione e interpretazione del diritto dall'età romana alle esperienza moderne. Ricerche dedicate al Professor Filippo Gallo, III, Napoli 1997, pp. 75-96.

Di Pietro P., Girolamo Tiraboschi, Rimini 1996.

DI Renzo Villata G., voce Cotta Catelliano, in DBGI I, p. 601.

di Renzo Villata G., voce Giasone del Maino, in DBGI I, pp. 995-999.

di Renzo Villata G., Diritto canonico, ricchezza e... povertà nell'opera di Felino Sandei, in Der Einfluß der Kanonistik auf die europäische Rechtskultur, V. Das Recht der Wirtschaft, hrsg. von D. von Mayenburg, O. Condorelli, F. Roumy, M. Schmoeckel, Köln-WeimarWien 2016, pp. 145-171.

DionisotTi C., Filologia umanistica e testi giuridici fra Quattro e Cinquecento, in La critica del testo. Atti del secondo congresso internazionale della Società Italiana di Storia del Diritto, I, Firenze 1971, pp. 189-204.

Diplovatati Th., Liber de claris iuris consultis, pars posterior curantibus $F$. Schulz, H. Kantorowicz, G. Rabotti, Bononiae 1968 (= Studia Gratiana post octava Decreti saecularia. Collectanea historiae iuris canoni$c i$, curantibus I. Forchielli, A.M. Stickler, X).

Dolcini C., Velut aurora surgente. Pepo, il vescovo Pietro e l'origine dello Studium bolognese, Roma 1987.

Dolcini C., Pepo, Irnerio, Graziano. Alle origini dello 'Studium' di Bologna, in O. CAPITANI (a cura di), L'università a Bologna. Personaggi, momenti e luoghi dalle origini al XVI secolo, Cinisello Balsamo (Mi) 1987, pp. 17-27.

DolezaleK G., Repertorium manuscriptorum veterum Codicis Iustiniani, unter Mitarbeit von L. Mayali, Frankfurt am Main 1985.

DolezaleK G., The Lectura Codicis of Odofredus, recensio I, and Jacobus Balduini, in The two Laws. Studies in Medieval Legal History Dedicated to Stephan Kuttner, ed. by L. Mayali and S.A.J. Tibbetts, Washington D.C. 1990, pp. 97-120.

Dolezalek G.-Weigand R., Das Geheimnis der roten Zeichen. Ein Beitrag zur Paläographie juristischer Handschriften des zwölften Jahrhunderts, in "Zeitschrift der Savigny-Stiftung für Rechtsgeschichte. Kanonistische Abteilung", 100 (1983), pp. 143-199.

Dondi A., Notizie storiche ed artistiche del Duomo di Modena, Modena 1896. 
Dufour J.-Giordanengo G.-Gouron A., L'attrait des 'leges'. Note sur la lettre d'un moine victorin (vers 1124/1127), in «Studia et Documenta Historiae et Iuris», XLV (1979), pp. 504-529.

Dumas A., Testament, in Dictionnaire de Droit Canonique, VII, Paris 1965, coll. 1190-2000.

DuYNSTEE M., An unknown fourteenth century lecture of the Orléans school: Jean Nicot on book VI of the Code, in TR, 60/3 (1992), pp. 363-375.

Duynstee M., Nicot (Nicoti, alias de Tramilguer) Jean, in Dictionnaire historique des juristes français, $X I I^{e}-X X^{e}$ siècle, sous la direction de $\mathrm{P}$. Arabeyre, J.-L. Halpérin, J. Krynen, Paris 2007, pp. 590-591.

Ebel F., FiJAl A., Kocher G., Römisches Rechtsleben im Mittelalter. Miniaturen aus den Handschriften des Corpus Iuris Civilis, Heidelberg 1988.

Emili A., voce Fridericus de Scalis, in C.A.L.M.A. Compendium Auctorum Latinorum Medii Aevi (500-1500), a cura di M. Lapidge, F. Santi, III/5, Firenze 2011, pp. 582-584.

ERrera A., Il concetto di Scientia iuris dal XII al XIV secolo. Il ruolo della Logica platonica e aritstotelica nelle scuole giuridiche medievali, Milano 2003.

Facciolati J., Fasti Gymnasii Patavini, I, Patavii, typis Seminarii, apud Joannem Manfrè, 1757 (rist. an. Sala Bolognese 1978).

FARINACCI P., Variarum quaestionum et communium opinionum criminalium liber quintus, de falsitate et simulatione titulus decimus sextus [...], Lugduni, sumptibus Horatii Cardon, 1612.

Favino L., Giovanni da Capestrano ed il diritto civile, in SM, s. III, XXXVI/1 (1995), pp. 255-284.

Feenstra R.-Duynstee M., Un recueil de textes de l'école de droit d'Orléans dans la Huntington Library à San Marino (Cal.), in TR, 60 (1992), pp. 81-108.

Feo G.-IAnnacci L.-Zuffrano A., Il formulario del documento privato tra norma giuridica e prassi notarile. L'apporto della scuola bolognese di notariato del secolo XIII, relazione presentata al congresso Les formulaires. Compilation et circulation des modèles d'actes dans l'Europe médiévale et moderne - XIII ${ }^{\mathrm{e}}$ congrès de la Commission internationale de diplomatique (Paris, 3-4 septembre 2012). Leggibile on-line alla URL < http://elec.enc.sorbonne.fr/cid2012/part7>.

Ferrari M., Il codex Muratorianus e il suo ultimo inedito, in IMU, 32 (1989), pp. 1-51. 
FICHARD J., Iurisconsultorum vitae veterum quidem, per Bernardinum Rutilium una cum eiusdem Decuria, recentiorum vero ad nostra usque tempora, per Ioannem Fichardum Francofurtensem [...], Basileae 1539.

Fichard J., Vitae recentiorum iurisconsultorum [...], Patauii, apud Iacobum Iordanum, ad signum Aquilae, 1565.

FIORI G., Osservazioni sulle famiglie nobili di Bobbio, in «Bollettino Storico Piacentino», LVII/1 (1962), pp. 23-27.

Fiori G., I podestà di Bobbio (sec. XIII-XVII), in «Bollettino Storico Piacentino», LVIII/2-3 (1963), pp. 97-103.

Fiori G., Le antiche famiglie di Piacenza e i loro stemmi, Piacenza 1979.

FIORI G., Le istituzioni ecclesiastiche minori bobbiesi: canonicati, cappellanie, benefici, congregazioni, oratori con un'appendice sulle sepolture delle antiche famiglie bobbiesi, in «Bollettino Storico Piacentino», XCVII/2 (2002), pp. 307-340.

FitTiNG H., Das Castrense Peculium in seiner geschichtlichen Entwicklung und heutigen gemeinrechtlichen Geltung, Halle 1871 (rist. an. Aalen 1969).

Fontana A., Amphiteatrum legale [...] seu bibliotheca legalis amplissima [...] quinque partibus divisa, Parmae, typis Iosephi ab Oleo \& Hippolyti Rosati, 1688 (rist. an. Torino 1961).

Fontana A., Bibliothecae legalis amplissimae continuatio, Parmae, typis haeredum Galeatii Rosati, 1694 (rist. an. Torino 1961).

Forciroli F., Vite dei modenesi illustri, a cura di S. Cavicchioli, trascrizione di G. Mancini, Modena 2007.

ForNilli C.C., Giambattista Scanaroli un modenese da conoscere, un apostolo delle carceri romane (1579-1665), Roma 1970.

Fornilli C.C., Delinquenti e carcerati a Roma alla metà del '600. Opera dei papi nella riforma carceraria, Roma 1991.

Fortunati M., Scrittura e prova. I libri di commercio nel diritto medievale e moderno, Roma 1996.

Fowler L., Forms of Arbitration, in Proceedings of the fourth international congress of medieval canon law, Toronto, 21-25 August 1972, ed. by S. Kuttner, Città del Vaticano 1976, pp. 133-147.

FranchinI V., L'istituto dei «Memoriali» in Bologna nel secolo XIII, in «L’Archiginnasio», IX (1914), pp. 95-106.

FrancIA V., Il contratto di soccida nel Bolognese nei secoli XIII e XIV, in «Archivio Giuridico», 77/1 (1922), pp. 68-93.

FREYMON J.W., Elenchus omnium auctorum sive scriptorum qui in iure tam civili quam canonico [...] claruerunt [...], Francofurti ad Moenum, apud Georgium Corvinum, 1574. 
Freymon J.W., Symphonia juris utriusque chronologica [...], Francofurti, ex officina typographica Nicolai Bassaei, 1574.

Fried J., Die Entstehung des Juristenständes im 12. Jahrhundert. Zur sozialen Stellung und politischen Bedeutung gelehrter Juristen in Bologna und Modena, Köln 1974.

Friederichs H.F, voce Fichard, Johann von, in Neue Deutsche Biographie, V, Berlin 1961, pp. 120-121.

FrIES J.J., Bibliotheca instituta et collecta primum a Conrado Gesnero, deinde in Epitomen redacta et novorum librorum accessione locupletata [...], Tiguri, apud Christophorum Froschoverum, 1583.

Fueter E.K., voce Gesner Konrad, in Neue Deutsche Biographie, 6, Berlin 1964, pp. 342-345.

Frigerio A., Martino da Fano e $i$ De modo studendi nelle università medievali, in V. Piergiovanni (a cura di), Medioevo notarile. Martino da Fano e il Formularium super contractibus et libellis, Milano 2007, pp. 57-67.

Frigerio A., Umanesimo del diritto: il De modo in iure studendi di Giovanni Battista Caccialupi (1467), in «Annali dell'Istituto storico italogermanico in Trento», XXX (2004), pp. 35-48.

Frugoni C. (a cura di), Il Duomo di Modena. The Cathedral of Modena. Testi. Text, Modena 1999.

Frugoni C. (a cura di), Il Duomo di Modena. The Cathedral of Modena. Atlante fotografico. Photo-Atlas, Modena 1999.

Galasso G., Il Regno di Napoli. Il Mezzogiorno angioino e aragonese (1266-1494), Torino 1992 (Storia d'Italia UTET, XV/1).

Gallo D., Università e signoria a Padova dal XIV al XV secolo, Trieste 1998.

GANDINO A., Quaestiones statutorum, in Scripta anecdota glossatorum vel glossatorum aetate composita (Bibliotheca iuridica medii aevi, III), Bononiae 1901, pp. 155-214.

García y García, A., Iter hispanicum, Firenze 1973.

Gargan L., L'enigmatico «conduxit». Libri e dogana a Padova fra Tre e Quattrocento, in QSUP, 16 (1983), pp. 1-41.

Gargan L., Nuovi codici "condotti” a Padova nel Tre e Quattrocento, in QSUP, 22-23 (1989-90), pp. 1-27.

GARGAN L., Le note «conduxit». Libri di maestri e studenti nelle università italiane del Tre e Quattrocento, in Manuels, programmes de cours et techniques d'enseignement dans les universités médiévales. Actes du colloque international de Louvain-la-Neuve (9-11 septembre 1993), ed. par J. Hamesse, Louvain-la-Neuve 1994, pp. 385-400. 
GaRgan L., Un nuovo elenco di note «conduxit»: la circolazione del libro universitario a Padova nel Tre e Quattrocento, in G.P. BrizzI, M.G. TAVONI (a cura di), Dalla pecia all'e-book. Libri per l'Università: stampa, editoria, circolazione e lettura. Atti del Convegno internazionale di studi. Bologna, 21-25 ottobre 2008, Bologna 2009, pp. 69-76.

GARDONI G., Per la biografia di Bovetino da Mantova decretorum doctor nello studio padovano $(†$ 1301), in QSUP, 37 (2004), pp. 3-30.

GaSSNER B., Nomenclatura doctorum in utroque iure et canonico et civili, passim per compendia literarum citatorum [...], Augustae Vindelicorum, in officina Henrici Steyneri, 1543.

GazzetTI E.-BARBiERI A., Cardinali, vescovi e abati nella storia della diocesi di Modena e Nonantola (sec. IV-sec. XX), S. Pietro in Cariano (VR) 1993.

Gesner C., Pandectarum sive partitionum universalium Conradi Gesneri Tigurini [...], Tiguri, excudebat Christophorus Froschouerus, 1548.

GESNER C., Bibliotheca universalis, sive catalogus omnium scriptorum locupletissimus [...] autore Conrado Gesnero Tigurino doctore medico, Tiguri, apud Christophorum Froschouerum, 1545 (rist. an. Konrad Gesner, Bibliotheca universalis und Appendix. Mit Nachwort von Prof. Dr. Hans Widmann, 2 voll., Osnabr ck 1966).

Ghirardacci C., Della Historia di Bologna parte prima, in Bologna, per Giovanni Rossi, 1596.

Ghisalberti C., La condanna al bando nel diritto comune, in «Archivio Giuridico», 158/1-2 (1960), pp. 3-75.

Giazzi E., voce Riccardo Malombra, in Autographa, I.2., pp. 66-72.

Giazzi E., voce Alberico da Rosciate, in Autographa, I.2., pp. 86-92.

GinanNI P.P., Memorie storico-critiche degli scrittori ravennati, II, in Faenza, presso Gioseffantonio Archi, 1769.

Giordanengo G., Les feudistes (XII ${ }^{e}-X V^{e}$ s.), in El dret comú $i$ Catalunya. Actes del II ${ }^{\text {on }}$ Simposi internacional, Barcelona 31 maig - juni de 1991, edició d’A. Iglesia Ferreirós, Barcelona 1992, pp. 67-139.

Giordanengo G., Jean Blanc feudiste de Marseille, (XIII ${ }^{e}$ siècle), in ID., Féodalités et droit savants dans le Midi médiéval, Aldershot-Brookfield 1992, pp. 71-93 (ed. orig. 1978).

GioRdanengo G., Le droit féodal dans les pays de droit écrit. L'exemple de la Provence et du Dauphiné, XII -début XIVe siècle, Rome 1988.

Giordanengo G., voce Blanc (Blancus) Jean, in Dictionnaire historique des juristes français, $X I I^{e}-X X^{e}$ siècle, sous la direction de P. Arabeyre, J.-L. Halpérin, J. Krynen, Paris 2007, p. 86 s.

Giovanni D'ANDREA, In secundum Decretalium librum Novella commenta- 
ria, Venetiis, apud Franciscum Franciscium Senensem, 1581 (rist. an. Torino 1963).

Giovanni D'Andrea, In titulum de Regulis iuris Novella Commentaria, Venetiis, apud Franciscum Franciscium senensem, 1581 (rist. an. Torino 1966).

Giovanni D’Andrea, Novella in Sextum, Venetiis, Philippus Pincius, 1499 (rist. an. Graz 1963).

Gloria A., Monumenti della Università di Padova (1222-1318), Venezia 1884 (rist. an. Bologna 1972).

Gnan P.-Toniolo F. (a cura di), Splendore nella Regola. Codici miniati da monasteri e conventi nella Biblioteca universitaria di Padova, RomaPadova 2011.

Gómez L., Index librorum omnium qui in vtroque iure hinc inde eduntur [...], Venetijs, per Gregorium de Gregoriis, 1525.

Greci R., Note sul commercio del libro universitario a Bologna nel Due e Trecento, in Studi di storia medioevale e di diplomatica, 9, Bologna 1987, pp. 49-97.

Gualazzini U., Contributi alla storia della scuola giuridica cremonese nel XII e XIII secolo, in Studi di storia e diritto in onore di Arrigo Solmi, I, Milano 1940, pp. 67-114.

Gualazzini U., Nuovi contributi per la storia dello «Studium» di Cremona nel Medioevo, in «Bollettino Storico Cremonese», 27 (1975-77), pp. 99-120.

Guglielmo da Pastrengo, De viris illustribus et De originibus, a cura di G. Bottari, Padova 1991 (Studi sul Petrarca, 21).

Guglielmo Durante, Speculum iudiciale, illustratum et repurgatum a Giovanni Andrea et Baldo degli Ubaldi, 2 tomi, Aalen 1975 (rist. an. dell'ed. Basileae, apud Ambrosium et Aurelium Frobenios fratres, 1574)

Guidi PAnZIRoli, De claris legum interpretibus libri quatuor, cura D. Christiani Godofredi Hoffmanni, Lipsiae, apud Jo. Frid. Gleditschii B. Filium, 1721.

Guidi PAnZIRoli, De claris legum interpretibus libri quatuor, Venetiis, apud Marcum Antonium Brogiollum, 1637.

HaAn B. (a cura di), Correspondance du nonce en France Gasparo Silingardi, évêque de Modène, 1599-1601, Rome 2002.

Henrici de Segusio cardinalis Hostiensis, Summa aurea, ad vetustissimos codices summa fide diligentiaque nunc primum collata [...], Basileae, apud Thomam Guarinum, 1573. 
Halm K.-Meyer G., Catalogus codicum latinorum Bibliothecae Regiae Monacensis, t. II, p. IV. Codices num. 21406-27268 complectens, Monachii 1881.

Hefele C.-J.-Leclerco H., Histoire des conciles, VI/1, Paris 1914.

Houben H., I Normanni, Bologna 2013.

[Iacobi Philipi Bergomensis], Supplementum chronicarum orbis ab initio mundi usque ad annum 1482, Venetiis 1483.

IACOPO BotTRIGARI, In primam et secundam veteris digesti partem, Romae, Typis Lepidi Fatii, 1606 (rist. an. Sala Bolognese 1978).

Iacopo D'Arena, Tractatus de bannitis, in TUI, XI/1, Venetiis 1584, ff. 355rb-357ra.

Iacopo d'Arena, Commentarii in universum ius civile [...], s.n.t. 1541 (rist. an. Bologna 1971).

Iannacci L., Modesti M., Zuffrano A., La misteriosa scrittura grande dei papiri ravennati, tra prassi documentaria pubblica e legislazione, in «Legal Roots», 1 (2012), pp. 89-119.

IASONIS MAYNI, In secundam digesti novi partem commentaria, Venetiis, apud Iuntas, 1585.

IOANNIS DE IMOLA, In primam Infortiati partem commentaria [...], Venetiis [Società dell'Aquila che si rinnova] 1580.

IOANNIS DE IMOLA, In secundam Infortiati partem commentaria [...], Venetiis [Società dell'Aquila che si rinnova] 1580.

IoANNIS DE IMOLA, In secundam Digesti novi partem commentaria [...], Venetiis [Società dell'Aquila che si rinnova] 1580.

IoANNIS AB Imola, In secundum Decretalium commentaria [...], Venetiis, apud Dominicum Nicolinum, 1575.

IsotTon R., voce Galeotti Alberto, in DBGI I, pp. 929-930.

Iurisconsultorum vitae, veterum quidem, per Bernardinum Rutilium una cum eiusdem Decuria, recentiorum verum ad nostra usque tempora per Ioannem Fichardum Francofurtensem, ad haec indices duo locupletissimi omnium scriptorum in iure tam pontificio quam civili [...], Basileae, per Ioannem Oporinum, 1539.

Johannes Jacobus Canis, De modo studendi in utroque iure, P[adua], $\mathrm{B}$ [artholomaeus de] V[aldezoccho], 1476.

Johannes Jacobus Canis, De executoribus ultimarum voluntatum, Constantiae, ex officina Leonhardi Straub., 1593 (già in TUI, VIII/1, cc. 185va-194vb).

Iohannis IAcobi CANis, De modo studendi in iure libellus. Nach der editio princeps vom Jahre 1476 hrsg. von G. Pescatore, Berlin 1889. 
Jung R., Dr. Johann Fichard, 1512-1581, in «Archiv für Frankfurts Geschichte und Kunst», s. III, 2 (1889), pp. 209-259.

Kantorowicz H.-Schulz F., De claris iuris consultis, 1. Lebensgeschichtliche Einleitung, Berlin und Leipzig 1919.

KARG A., Fichard Johann, in Handwörterbuch zur deutschen Rechtsgeschichte, I, Berlin 2008 (Zweite völlig überarbeitete und erweiterte Auflage), pp. 1570-1572.

KleINHEYER G.-SchröDER J., Deutsche Juristen aus fünf Jahrhunderten, Heidelberg 1990.

Kohl B.G., voce Lovati Lovato, in DBI, 66 (2006), pp. 215-220.

Kuttner S., Elze R. (a cura di), A Catalogue of Canon and Roman Law Manuscripts in the Vatican Library, I. Codices Vaticani latini 5412299, Città del Vaticano 1986; II. Codices Vaticani latini 2300-2746, Città del Vaticano 1987.

Labardi A., voce Malombra Riccardo (a), in DBI, 68 (2007), pp. 261-264.

LABARDi A., voce Malombra Riccardo (b), in DBGI, II, p. 1237.

LABARDi A., Mattarelli Niccolò, in DBI, 72, Roma 2009, pp. 101-103.

Legendre P., Manuscrits avec gloses post-accursiennes, in «Revue Historique de Droit français et étranger», s. IV, 42 (1964), pp. 702-704.

Lehmann K. (a cura di), Consuetudines feudorum, II ed., Aalen 1971.

LePsius S., voce Bartolo da Sassoferrato, in DBGI, I, pp. 177-180.

Liber Regiminum Padue, a cura di A. Bonardi, in RIS, II ed., VIII/1, Città di Castello 1903, pp. 267-376.

LiotTa F., Martino da Fano giurista e pratico del diritto nell'Italia del XIII secolo, in V. Piergiovanni (a cura di), Medioevo notarile. Martino da Fano e il Formularium super contractibus et libellis, Milano 2007, pp. 1-5.

Liotta F., voce Del Cassero Martino, in Dizionario Biografico degli Italiani, 36, Roma 1988, pp. 442-46.

LIPEN M., Biblioteca realis iuridica [...], Lipsiae, apud Fridericum Matthiam Frisium, 1736.

Lorenzoni G., Prime ricerche sulla famiglia Grassoni tra Vignola e Modena (XII-XIV secolo), in V. Braidi, G. Lorenzoni, Consorterie nobiliari sul confine tra Modena e Bologna. I Boccadiferro e i Grassoni (secc. XI-XIV), Modena 2003, pp. 173-293.

Loschiavo L., voce Morisio Omobono, in DBI, 76, Roma 2012, pp. 863866. 
Loschiavo L., voce Omobono Morisio, in DBGI, II, pp. 1455-1456.

Lucchesi C., Manoscritti della Biblioteca Comunale dell'Archiginnasio di Bologna contenenti opere di lettori dello Studio di Padova, in «Studi e memorie per la storia dell’Università di Bologna», 7 (1922), pp. 33-54.

Lucchi G., "Camera Segreta". Codici statutari, registri ed atti conoscitivi della Comunità e delle arti. Inventario, Modena 1963.

Macino F., Contributo alla storia della trasmissione del Tractatus super instrumentis di Niccolò Mattarelli, in U. Petronio, O. Diliberto (a cura di), Scritti di storia del diritto e bibliografia giuridica offerti a Giuliano Bonfanti, Macerata 2012, pp. 257-265.

Maffer D., Baldo e Alberto Guidalotti fra Perugia e Padova con altre notizie, in C. Frova, M.G. Nico Ottaviani, S. Zucchini (a cura di), VI centenario della morte di Baldo degli Ubaldi 1400-2000, Perugia 2005, pp. 17-24.

MAFFEI D., Qualche postilla alle ricerche di E.M. Meijers sulle scuole di Orléans, Tolosa e Montpellier, in ID., Studi di storia delle università e della letteratura giuridica, Goldbach 1995, pp. 89-102 (già in TR, 36 (1968), pp. 387-400).

MAFFeI D., Un trattato inedito di Bonaccorso degli Elisei e i più antichi statuti dello Studio di Bologna nel manoscritto 22 della Robbins Collection, in ID., Studi di storia delle università e della letteratura giuridica, Goldbach 1995, pp. 23-51 (già in «Bulletin of Medieval Canon Law», 5 (1975), pp. 73-101).

MAFFeI D., Di un inedito «De modo in iure studendi» di Diomede Mariconda. Con notizie su altre opere e lo Studio di Napoli nel Quattrocento in ID., Studi di storia delle università e della letteratura giuridica, Goldbach 1995, pp. 299-321 (già in RIDC 2 (1991), pp. 7-29).

Maffer D., Giovan Battista Caccialupi biografo, in «Zeitschrift der Savigny-Stiftung für Rechtsgeschichte. Kanonistiche Abteilung», LXXXIII (1997), pp. 392-400.

Maffer D., Osberto da Cremona a Vercelli e una sua quaestio sul giudice testimone, in Iuris Historia. Liber Amicorum Gero Dolezalek, ed. by V. Colli and E. Conte, Berkeley 2008, pp. 315-321.

Maffer P., voce Cino Sinibuldi da Pistoia, in DBGI, I, pp. 543-546.

Maffer P., voce Mariconda Diomede, in DBGI, II, p. 1275.

MaIre Vigueur J.-C., Religione e politica nella propaganda pontificia (Italia comunale, prima metà del XIII secolo), in P. CAMmAROSANO (a cura di), Le forme della propaganda politica nel Due e Trecento, Roma 1994, pp. 65-83. 
Mantovani G.P., Le orazioni accademiche per il dottorato: una fonte per la biografia degli studenti? Spunti dal caso padovano, in F. Piovan, L. Sitran Rea (a cura di), Studenti, università, città nella storia padovana. Atti del convegno, Padova, 6-8 febbraio 1998, Trieste 2001 (Contributi alla storia dell'Università di Padova, 34), pp. 73-115.

Marangon P., Gli «Studia» degli ordini mendicanti, in ID., Ad cognitionem scientiae festinare. Gli studi nell'Università e nei conventi di Padova nei secoli XIII e XIV, a cura di T. Pesenti, Trieste 1997 (Contributi alla storia dell'Università di Padova, 31), pp. 70-114 (già edito in Storia e cultura a Padova nell'età di sant'Antonio, Padova 1985, pp. 344-380).

Marcello A., Opere giustamente e ingiustamente attribuite a Jacopo d'Arena, in «Rendiconti del Reale Istituto Lombardo di Scienze e Lettere», s. II, 61 (1928), pp. 850-861.

Marchetti P., Inventario dell'Archivio Notarile di Modena con prefazione storica sull" "Ufficio del Memoriale”, in G. Degli Azzi (a cura di), Gli Archivi della Storia d'Italia, s. II, v. III, Rocca S. Casciano 1913, pp. 1-66.

Marci Mantuae, Epitoma virorum illustrium qui vel scripserunt, vel iurisprudentiam docuerunt in scholis, \& quo tempore etiam floruerunt, ordine alphabetico constitutum [...], Patauij, Gratiosus Perchacinus excudebat, 1555.

MARI P., Un consiglio collegiale dei dottori pavesi nel primo Quattrocento, in RSDI, LXIII (1990), pp. 23-118.

MARI M., Il genio freddo. La storiografia letteraria di Girolamo Tiraboschi, Milano $1999^{2}$.

Marrara D., L'età medicea (1543-1737), in Storia dell'Università di Pisa, 1* - 1343-1737, Pisa 1993, pp. 79-187.

Marrella F.-Mozzato A., Alle origini dell'arbitrato commerciale internazionale. L'arbitrato a Venezia tra medioevo ed età moderna, Padova 2001.

Martino F., Ricerche sull'opera di Guido da Suzzara. Le «Supleciones», Catania 1981.

Martino F., Testimonianze sull'insegnamento del diritto a Napoli nei secoli XIII-XIV. Il manoscritto ambrosiano E. 29 inf., in M. Bellomo, Scuole, diritto e società nel Mezzogiorno medievale d'Italia, II, Catania 1987, pp. 25-38.

MARTINo F., «Lecturae per viam additionum» nel ms. 317 della Biblioteca Capitolare di Lucca, in «Quellen und Foschungen aus italienischen Archiven un Bibliotheken», 67 (1987), pp. 462-476.

Martino F., Argumenta ex legibus, in Die Kunst der Disputation. Probleme 
der Rechtsauslegung und Rechtsanwendung im 13. und 14. Jahrhundert, hrsg. von M. Bellomo, München 1997, pp. 147-156.

Martinoli L., Quattro frammenti di 'Libri magni quaestionum disputatarum', in RIDC, 15 (2004), pp. 269-280 (poi in M. AsCheri, G. CoLLI (a cura di), Manoscritti, editoria e biblioteche dal medioevo all'età contemporanea. Studi offerti a Domenico Maffei per il suo ottantesimo compleanno, II, Roma 2006, pp. 665-677).

Martinoli Santini L., Peruzzi P., Catalogo delle opere giuridiche contenute nel ms. Vittorio Emanuele 1511 della Biblioteca Nazionale Centrale di Roma, in RIDC, 7 (1996), pp. 217-315.

Martone L., Arbiter-arbitrator. Forme di giustizia privata nell'età del diritto comune, Napoli 1984.

Mattarelli N., Tractatus super instrumentis, a cura di F. Macino, Roma 2012.

Matteo Gribaldi Mofa, De methodo ac ratione studendi libri tres [...], Lugduni, apud A. Vincentium, 1541 (altra ed. apud Theobaldum Paganum, 1543).

Mattone A., Manuale giuridico e insegnamento del diritto nelle università italiane del XVI secolo, in «Diritto@Storia», 6 (2007), ed. on-line alla URL < http://www.dirittoestoria.it/6/Contributi/Mattone-Manualegiuridico-insegnamento-universit-XVI-secolo.htm $\geq$.

Mattone A., voce Solmi Arrigo, in DBGI II, pp. 1889-1892.

Mazzacane A., voce Diplovatazio Tommaso, in DBI, 40, Roma 1991, pp. 249-254.

Mazzacane A., voce Farinacci Prospero, in DBI, 45, Roma 1995, pp. 1-5.

Mazzanti G., voce Guido da Suzzara, in DBI, 61, Roma 2003, pp. 421-426.

Mazzanti G.-Murano G., voce Guido da Suzzara, in Autographa, 1.2., pp. 19-28.

Mazzatinti G., Inventari dei manoscritti delle biblioteche d'Italia, 79, Firenze 1954.

Meccarelli M., Arbitrium. Un aspetto distematico degli ordinamenti giuridici in età di diritto comune, Milano 1998.

Medica M., La Bibbia di Gerona e il suo committente: una proposta per il cardinale Jean Cholet, in «Arte Medievale», II/2 (2003), pp. 65-85.

Megli Frattini L., Foresti Giacomo Filippo, in Dizionario Biografico degli Italiani, 48, Roma 1997, pp. 801-803.

MeIJERs E.M., Etudes d'histoire du droit, III. Le droit romain au moyen age, a cura di R. Feenstra, H.F.W.D. Fischer, Leyde 1959.

Mellusi G.G., Giovanni d'Anguissola, in DBGI I, p. 1012. 
Menant F., L'Italia dei comuni (1100-1350), Roma 2011.

Menant F., Podestats et capitaines du people d'origine crémonaise, in J.-C. Maire Vigueur (a cura di), I podestà dell'Italia comunale, I. Reclutamento e circolazione degli ufficiali forestieri (fine XII sec.-metà XIV sec.), Roma 2000, pp. 75-105.

Menzinger S., voce Federico della Scala (delle Scale), in DBGI, I, p. 833.

Menzinger S., Vallerani M., Giuristi e città: fiscalità, giustizia e cultura giuridica tra XII e XIII secolo. Ipotesi e percorsi di ricerca, in M.T. Caciorgna, S. Carocci, A. Zorzi (a cura di), I comuni di Jean-Claude Maire Vigueur. Percorsi storiografici, Roma 2014, pp. 201-234.

Mercati A., Per la storia letteraria di Reggio Emilia, in AMDSPMo, s. V, XII (1919), pp. 37-116.

Mercati A., M. Tulli Ciceronis De re publica libri e codice rescripto vaticano latino 5757 [...], Città del Vaticano 1934.

Mercati S.G., Sul luogo e sulla data di composizione delle «Derivationes» di Uguccione da Pisa, in «Aevum», 33 (1959), pp. 490-494.

Merzbacher F., Freymon von Randeck, Johann Wolfgang, in Neue Deutsche Biographie, V, Berlin 1961, pp. 423-424.

Merzbacher F., Das Testamentsrecht des Corpus Iuris Canonici, in «Österreichische Archiv für Kirchenrecht», 19 (1968), pp. 289-307.

Meyer A., Felix et inclitus notarius. Studien zum italienischen Notariat vom 7. bis zum 13. Jahrhundert, Tübingen 2000.

Migliorino F., «Dominus meus in legibus»: the Search for a Liber quaestionum of Martinus Sillimani, in The Two Laws. Studies in Medieval Legal History dedicated to Stephan Kuttner, ed. by L. Mayali and S.A.J. Tibbetts, Washington D.C. 1990, pp. 121-151.

Milani G., Prime note sulla disciplina del bando a Bologna attorno alla metà del XIII secolo, in «Mélanges de l'Ecole française de Rome. Moyen-Age, Temps modernes», 109/2 (1997), pp. 501-523.

Milani G., L'esclusione dal Comune. Conflitti e bandi politici a Bologna e in altre città italiane tra XII e XIV secolo, Roma 2003.

Milani G., I comuni italiani. Secoli XII-XIV, Roma-Bari 2005.

Milani G., Banditi, malesardi e ribelli. L'evoluzione del nemico pubblico nell'Italia comunale (secoli XII-XIV), in I diritti dei nemici, Milano 2009, pp. 109-140 ("Quaderni Fiorentini per la storia del pensiero giuridico moderno", 38/I).

Missere Fontana F., Guido Panciroli numismatico e alcune annotazioni sul rapporto fra imprese e «medaglie antiche», in «Memorie dell'Accademia Italiana di Studi Filatelici e Numismatici», VI/I (1995), pp. 93-126. 
Montanos Ferrin E., «An de die vel de nocte», in RIDC, 9 (1998), pp. 49-80.

MontFaucon (DE) B., Bibliotheca bibliothecarum manuscriptorum nova, 2 voll., Parisiis, apud Briasson, via Jacobaea, ad Insigne Scientiae, 1739.

Montorzi M., Fides in rem publicam. Ambiguità e tecniche del diritto comune, Napoli 1984.

Montorzi M., L'«intellettualismo pratico» della Lectura super usibus feudorum di Baldo degli Ubaldi tra prassi forense e nuove sistemazioni dottorali (prove e problemi di lettura), in C. Frova, M.G. Nico OtTAVIANI (a cura di), VI centenario della morte di Baldo degli Ubaldi 14002000, S. Zucchini, Perugia 2005, pp. 205-261 (anche in ID., Processi istituzionali. Episodi di formalizzazione giuridica ed evenienze d'aggregazione istituzionale attorno ed oltre il feudo. Saggi e documenti, Padova 2005, pp. 290-338).

Montorzi M., voce Sandei Felino, in DBGI, II, pp. 1781-1783.

Montorzi M., Felino Sandei, in Autographa, I.1., pp. 273-275.

Mor C.G., La divisione in paragrafi delle leggi del Digesto (contributo alla storia della scuola di Bologna), in RSDI, $26-27$ (1953-54), pp. 145-162 (riedito in Scritti in memoria di Sergio Mochi Onory, Milano 1958, pp. 139-156).

Mor C.G.-Di Pietro P., Storia dell'Università di Modena, 2 voll., Firenze 1975.

Morelli G.-Murano G., voce Salatiele, in Autographa, I.1., pp. 22-26.

MüLler W.P., The Recovery of Justinian's Digest in the Middle Ages, in "Bulletin of Medieval Canon Law", 20 (1990), pp. 1-29.

MüLLER W.P., Signorolus de Homodeis and the medieval interpretation of statutory law, in RIDC, 6 (1995), pp. 217-232.

Murano G., Copisti a Bologna (1265-1270), Turnhout 2006.

Murano G., Firme e monogrammi d'artista. R magister e Bartolomeo Tinti da Modena, in «Rivista di storia della miniatura», 17 (2013), pp. 75-82.

Murano G., Paolo di Jacopino Avvocati (fl. 1251-1297, in G.P. BrizzI, M.G. TAVONI (a cura di), Dalla pecia all'e-book. Libri per l'Università: stampa, editoria, circolazione e lettura. Atti del Convegno internazionale di studi (Bologna, 21-25 ottobre 2000), Bologna 2009 (Centro interuniversitario per la storia delle università italiane. Studi, 11), pp. 13-31.

Murano G., voce Baldo degli Ubaldi, in Autographa, I.1., pp. 103-108.

Murano G., voce Cino da Pistoia, in Autographa, I.1., pp. 35-39.

Murano G., voce Felino Sandei (le opere e la biblioteca), in Autographa, I.1., pp. 276-282.

Murano G., voce Raffaele Fulgosio, in Autographa, I.1., pp. 145-147. 
Murano G., voce Rolandino Passaggeri, in Autographa, I.1., pp. 27-31.

Murano G., voce Guillaume Durand (Guglielmo Durante), in Autographa, I.2., pp. 49-53.

Murano G., voce Dino del Mugello, in Autographa, I.2., pp. 54-65.

Murano G., voce Oldrado da Ponte, in Autographa, I.2., pp. 80-85.

Murano G., voce Martino Garati da Lodi, in Autographa, I.2., pp. 187191.

Muzzarelli M.G., Candelabrum lucem ferens. Il prestito del Monte di Pietà nel pensiero dei giuristi Benedetto capra e Baglione dei Montevibia$n i$, in D. Quaglioni, G. Todeschini. G.M. Varanini (a cura di), Credito e usura fra teologia, diritto e amministrazione. Linguaggi a confronto (sec. XII-XVI), Roma 2005, pp. 181-196.

Najemy J.M., Storia di Firenze, 1200-1575, Torino 2014 (ed. orig. 2006).

Nardi P., Giovanni Battista Caccialupi a Siena: giudice delle Riformazioni e docente nello Studio, in «Studi Senesi», CIX (1997), pp. 83-124.

Nardi P., Martino da Fano e la sua attività di docente nello Studium di Arezzo, in V. Piergiovanni (a cura di), Medioevo notarile. Martino da Fano e il Formularium super contractibus et libellis, Milano 2007, pp. 19-30.

NARducci E., Catalogus codicum manuscriptorum praeter Graecos et Orientales in Bibliotheca Angelica olim coenobii Sancti Augustini de Urbe, Tomus prior [mss. 1-1543], Romae 1893.

Nicolaj G., Lineamenti di diplomatica generale, in «Scrineum Rivista», I (2003), ed. on-line alla URL <http://www.fupress.net/index.php/scrineum/article/view/12094>.

Nicolaj G., Documenti e libri legales a Ravenna: rilettura di un mosaico leggendario, in Ravenna da capitale imperiale a capitale esarcale. Atti del XVII Congresso internazionale di studio sull'alto medioevo, Ravenna, 6-12 giugno 2004, II, Spoleto 2005, pp. 761-799.

Nicolaj G., Lezioni di diplomatica generale, I. Istituzioni, Roma 2007.

Nicolini U., Trattati 'de positionibus' attribuiti a Martino da Fano, Milano 1935.

Nicolini U., I giuristi postaccursiani e la fortuna della glossa in Italia, in G. Rossi (a cura di), Atti del Convegno Internazionale di Studi Accursiani, III, Milano 1968, pp. 799-943 (in versione più breve e priva di note in ID., Scritti di storia del diritto italiano, Milano 1983, pp. 336-363).

Nuovo A., I Giolito e l'editoria giuridica del XVI secolo, in M. AscherI, G. Colli (a cura di), Manoscritti, editoria, biblioteche dal medioevo all'e- 
tà contemporanea. Studi offerti a Domenico Maffei per il suo ottantesimo compleanno, III, Roma 2006, pp. 1019-1051.

Nuovo A., Produzione e circolazione di libri giuridici tra Italia e Francia (sec. XVI): la via commerciale Lione-Trino-Venezia, in G.P. BRIzzI, M.G. TAVONI (a cura di), Dalla pecia all'e-book. Libri per l'Università: stampa, editoria, circolazione e lettura. Atti del Convegno internazionale di studi (Bologna, 21-25 ottobre 2000), Bologna 2009 (Centro interuniversitario per la storia delle università italiane. Studi, 11), pp. 341-349.

Odofredi, In primam Codicis partem Praelectiones, Lugduni, excudebant Franciscus et Claudius Marchant fratres, 1552 (rist. an. Bologna 1968).

OldRado da Ponte, Consilia seu responsa et quaestiones aureae, Venetiis, apud Franciscum Zilettum, 1570.

OrLandelli G., Appunti sulla scuola bolognese di notariato nel secolo XIII per un'edizione della «Ars notariae» di Salatiele, in Studi e Memorie per la storia dell'Università di Bologna, II, Bologna 1961, pp. 1-54.

Orlandelli G., Genesi dell' «ars notariae» nel secolo XIII, in SM, s. III, VI/ II (1965), pp. 329-366.

Orlandelli G., I Memoriali bolognesi come fonte per la storia dei tempi di Dante, in Dante e Bologna nei tempi di Dante, Bologna 1967, pp. 191-205 (ristampato in ID., Scritti di paleografia e diplomatica, a cura di R. Ferrara-G. Feo, Bologna 1994, pp. 77-91).

OsLer D., Towards a legal-historical Bibliography: a Census of 16th Century Legal Imprints, in IC, 15 (1988), pp. 231-242.

Pace G., 'Cosmas de Veronisiis de Sicilia' studente a Padova, in RIDC 1 (1990), pp. 193-199.

Pace G., 'Garnerius Theutonicus'. Nuove fonti si Irnerio e i 'quattro dottori', in RIDC, 2 (1991), pp. 123-133 (e in Miscellanea Domenico Maffei dicata. Historia - Ius - Studium, I, Goldbach 1995, pp. 91101).

PaCE G., Riccardo da Saliceto. Un giurista bolognese del Trecento, Roma 1995.

Padoa Schioppa A., Martino da Fano processualista. Note sul Formularium, in V. Piergiovann (a cura di), Medioevo notarile. Martino da Fano e il Formularium super contractibus et libellis, Milano 2007, pp. 67-81 (riedito in Iuris Historia. Liber Amicorum Gero Dolezalek, ed. by V. Colli and E. Conte, Berkeley 2008, pp. 281-292). 
Padovani A., L'archivio di Odofredo. Le pergamene della famiglia Gandolfi Odofredi. Edizione e regesto (1163-1499), Spoleto 1992.

Padovani A., The 'Additiones et apostillae super secunda parte Infortiati' of Cinus de Pistoia, in The two Laws. Studies in medieval legal history dedicated to Stephan Kuttner, ed. by L. Mayali and S.A.J. Tibbetts, Washington 1990, pp. 152-165.

Padovani A., Studi storici sulla dottrina delle sostituzioni, Milano 1983.

Padovani A., A proposito di Manlio Bellomo, I fatti e il diritto. Tra le certezze e i dubbi dei giuristi medievali (secoli XIII-XIV), Roma, Il Cigno Galileo Galilei, 2000, pp. 7-750, in RSDI, LXXIV-LXXV (20012002), pp. 431-452.

Padovani A., Modernità degli antichi. Breviario di argomentazione forense, Bologna 2006.

Padovani A., voce Giovanni da Imola, in Autographa, I.1., pp. 155-164.

Padovani A., voce Dino Rossoni del Mugello, in DBGI, I, pp. 769-771.

Padovani A., Sette orationes pavesi pro doctoratu di Baldo degli Ubaldi, in B. Pio, R. Parmeggiani (a cura di), L'università in tempi di crisi. Revisioni e novità dei saperi e delle istituzioni nel Trecento, da Bologna all'Europa, Bologna 2016, pp. 27-61.

Padovani A., Giustizia e lavoro nelle Summae di Antonino da Firenze, Angelo da Chivasso e Giovanni Battista Trovamala, in Der Einfluß der Kanonistik auf die europäische Rechtskultur, V. Das Recht der Wirtschaft, hrsg. von D. von Mayenburg, O. Condorelli, F. Roumy, M. Schmoeckel, Köln-Weimar-Wien 2016, pp. 375-403.

Padovani A., Un sermo di Cino da Pistoia dal ms. Biblioteca Vaticana Chigi E.VIII.245, in RIDC, 27 (2016), pp. 11-41.

Padovani A., Alle origini dell'Università di Bologna: l'insegnamento di Irnerio, in «Bulletin of Medieval Canon Law», 33 (2016), pp. 13-25.

Padovani A., voce Giovanni Nicoletti (de Calcis) da Imola, in DBGI, I, pp. 1015-1018.

Padovani A., Dall'alba al crepuscolo del commento. Giovanni da Imola (1375 ca.-1436) e la giurisprudenza del suo tempo, Frankfurt am Main 2017.

Palmieri G.B. (a cura di), Pillii libellus de preparatoriis litium et earum preambulis ex cod. Ms. Cassinensi XX 213, in Scripta anecdota glossatorum vel glossatorum aetate composita (Bibliotheca iuridica medii aevi, III), Bononiae 1901, pp. 15-68.

Panini F., Cronica della Città di Modona, a cura di R. Bussi, R. Montagnani, Modena 1978. 
Papadopoli Nicolai Comneni, Historia gymnasii patavini, post ea, quæ hactenus de illo scripta sunt, ad hæc nostra tempora pleniùs et emendatius deducta. Tomus I, Venetiis, apud Sebastianum Coleti, 1726.

Papias Vocabulista, Elementarium doctrinae erudimentum, Venetiis, Philippus Pincius, 1496 (rist. an. Torino 1966).

Papillon Ph., Bibliothéque des auteurs de Bourgogne, II, Dijon, Philippe Marteret, 1742.

PARMeggiani R., Visite pastorali e riforma a Bologna durante l'episcopato di Niccolò Albergati (1417-1443), in «Rivista di Storia della Chiesa in Italia», 59/1 (2015), pp. 21-47.

Pasolini S., Lustri ravennati [...] parte seconda, in Bologna, per Giacomo Monti, 1679.

PAsolini S., Lustri ravennati [...] parte terza, in Bologna, per Giacomo Monti, 1680.

Pasolini S., Huomini illustri di Ravenna antica et altri degni professori di lettere e armi, in Bologna, per Pier Maria Monti, 1703.

Paulus de Castro, Prima super Codice [...], Lugduni [Vincent de Portonariis] 1543.

Paulus de Castro, Super Codice pars I, Venetiis [al segno della Corona] 1550.

Paulus de CAStro, Consiliorum siue responsorum [...] volumen primum [...], Venetiis, [Società dell'Aquila che si rinnova], 1580.

Pazzaglini P.R., The criminal ban of the Sienese Commune, 1225-1310, Ann Arbor 1986.

Pecorella C., Fides pro se, in «Studi Parmensi», XXII (1978), pp. 131-231 (riedito in ID., Studi e ricerche di storia del diritto, Torino 1995, pp. 373-450).

Pecorella C., Studi sul notariato a Piacenza nel sec. XIII, Milano 1968.

Peruzzi P., Prime note sulla vita e sull'opera scientifica di Francesco Tigrini da Pisa, in SM, s. III, XXXI/II (1990), pp. 853-899.

Peruzzi P., Da un manoscritto miscellaneo ad una raccolta di «quaestiones». Prima descrizione del ms. Vittorio Emanuele 1511 della Biblioteca Nazionale di Roma, in Die Kunst der Disputation. Probleme der Rechtsauslegung und Rechtsanwendung im 13. und 14. Jahrhundert, hrsg. von M. Bellomo, München 1997, pp. 205-215.

Peruzz P., Tigrini (Tigrino) Francesco, in DBGI, II, pp. 1951-1952.

Pescani P., La posizione del V 1406 nella ricostruzione della prima parte del Digesto, in Studi in onore di Giuseppe Grosso, V, Torino 1972, pp. 81-111. 
Pescani P., La scoperta del "Bononiensis". Sue forme, in "Bullettino dell'Istituto di Diritto Romano Vittorio Scialoja”, s. III, XXVII (1985), pp. 383-396.

Pescatore G. (a cura di), Thomae Diplovatatii opus de praestantia doctorum. Erste abteilung, Berlin 1890.

Petitjean M., voce Taisand Pierre, in Dictionnaire historique des juristes français $\left(X I I^{e}-X X^{e}\right.$ s. ), a cura di P. Arabeyre, J.-L. Halpérin, J. Krynen, Paris $2015^{2}$.

Petitmengin P., Montfaucon, dom Le Maitre et la Bibliotheca Bibliothecarum, in Du copiste au collectionneur. Mélanges d'histoire des textes et des bibliothèques en l'honneur d'André Vernet, textes réunis par D. Nebbiai-Dalla Guarda, J.-F. Genest, Turnhout 1998, pp. 537-584.

Petoletti M., «Ad utilitatem volentium studere in ipsa comedia»: il commento dantesco di Alberico da Rosciate, in IMU, 38 (1995), pp. 141-216.

Petti Balbi G., Martino da Fano, podestà a Genova, in V. Piergiovanni (a cura di), Medioevo notarile. Martino da Fano e il Formularium super contractibus et libellis, Milano 2007, pp. 7-17.

Petti Balbi G., Cultura e potere a Genova: la biblioteca di Raffaele Adorno (1396), in EAD., Governare la città. Pratiche sociali e linguaggi politici a Genova in età medievale, Firenze 2007, pp. 247-260 (Reti Medievali. Monografie, 4).

Pezzella S., voce Carletti Angelo, in DBI, 20, Roma 1977, pp. 136-138.

Piana C., Nuove ricerche su le Università di Bologna e di Parma, Florentiae 1966.

Piano Mortari V., voce Arbitrato (diritto intermedio), in ED, II, Milano 1958, pp. 895-899.

Piccialuti M., voce Battista da Sambiagio, in DBI, 7, Roma 1970, pp. 253256.

Piergiovanni V., Un trattatello sui mercanti di Baldo degli Ubaldi, in M. Ascheri (a cura di), Scritti di storia del diritto offerti dagli allievi a Domenico Maffei, Padova 1991, pp. 235-254.

Piergiovanni V., Profili storici dal diritto romano al diritto medievale e moderno, in G. Alpa (a cura di), L'arbitrato. Profili sostanziali, Torino 1999, pp. 3-15.

Polizzi F., Nuovi documenti e ricerche sul cenacolo preumanistico padovano, in IMU, 28 (1985), pp. 137-181.

Pomaro G. (a cura di), I manoscritti medievali della Biblioteca capitolare feliniana di Lucca, Firenze 2015. 
Portenari A., Della felicità di Padova libri nove, in Padova, per Pietro Paolo Tozzi, 1627.

Post G., Masters' salaries and student-fees in the mediaeval universities, in «Speculum», 7/2 (1932), pp. 181-198.

Preto P., voce Colle Francesco Maria, in DBI, 26, Roma 1982, pp. 797-799.

Prosdocimi L., Alberico da Rosciate e la giurisprudenza italiana del secolo XIV, in «Bergomum», 29/4 (1956), pp. 1-7 (e in RSDI, 29 (1956), pp. 67-74).

Prosdocimi L., voce Alberico da Rosate, in DBI, 1, Roma 1960, p. 656 s.

Prosdocimi L., voce Bellencini Bartolomeo, in DBI, 7, Roma 1965, p. 629.

Puncuh D., Rovere A. (a cura di), I Libri Iurium della Repubblica di Genova. Introduzione, Roma 1992 (Pubblicazioni degli Archivi di Stato. Fonti, XII).

Quaglioni D., Politica e diritto nel Trecento italiano. Il «De tyranno» di Bartolo da Sassoferrato (1314-1357). Con l'edizione critica dei trattati «De Guelphis et Gebellinis», «De regimine civitatis»e «De tyranno», Firenze 1983.

Quaglioni D., Legislazione statutaria e dottrina della legislazione: le «quaestiones statutorum» di Alberico da Rosciate, in ID., «Civilis sapientia». Dottrine giuridiche e dottrine politiche fra medioevo ed età moderna. Saggi per la storia del pensiero giuridico moderno, Rimini 1989, pp. 35-75.

Quaglioni D., voce Caccialupi Giovanni Battista, in DBGI, I, pp. 369-370.

Quaglioni D., voce Gandino Alberto, in DBGI, I, pp. 942-944.

Quaglioni D., voce Dell'Arena (D'Arena) Jacopo, in DBI, 37, Roma 1989, pp. 243-250.

Quaglioni, voce Iacopo d'Arena, in DBGI, I, pp. 1099-1101.

Quaglioni D., voce Delle Scale Federico, in DBI, 38, Roma 1990, pp. 5560.

Quaglioni D., voce Gribaldi Moffa Matteo, in DBI, 59, Roma 2002, pp. 345-349.

Quaglioni D., Autosufficienza e primato del diritto nell'educazione giuridica preumanistica, in Sapere elè potere. Discipline, dispute e professioni nell'Università medievale e moderna. Il caso bolognese a confronto. Atti del $4^{\circ}$ Convegno, Bologna, 13-15 aprile 1989, II. Verso un nuovo sistema del sapere, a cura di A. Cristiani, Bologna 1990, pp. 125-134.

Quaglioni D., Il pubblico dei legisti trecenteschi: i 'lettori' di Bartolo, in M. Ascheri (a cura di), Scritti di storia del diritto offerti dagli allievi a Domenico Maffei, Padova 1991, pp. 181-201. 
Quaglioni D., Standum canonistis? Le usure nella dottrina civilistica medievale, in D. Quaglioni, G. Todeschini, G.M. Varanini (a cura di), Credito e usura fra teologia, diritto e amministrazione. Linguaggi a confronto (sec. XII-XVI), Roma 2005, pp. 247-264.

Radding C.M.-Ciaralli A., The Corpus iuris civilis in the Middle Ages. Manuscripts and transmission from the sixth century to the juristic revival, Leiden-Boston 2007.

RagGi O., Elogio di Giovanni Battista Scanarolo, vescovo di Sidonia autore dell'opera sulla visita de' carcerati, Roma 1842.

Ranieri da Perugia, Ars Notariae, hrsg. von L. Wahrmund (Quellen zur Geschichte des römisch-kanonischen Prozessen im Mittelalter, III/II), Innsbruck 1917 (rist. an. Aalen 1962).

Raphaelis Cumani nempe et Fulgosil, Consilia sive responsa acutissimorum iuris interpretum, Venetiis, apud Gasparem Bindonum, 1575.

Ricci D., Di G.B. Scanaroli modenese, Arcivescovo di Sidone e di Tiro, Roma 1889.

Ricciardi R., voce Cani Giovanni Iacopo, in DBI, 18, Roma 1975, pp. 74-76.

Riccoboni A., De Gymnasio patavino commentariorum libri sex, Patavii, apud Franciscum Bolzetam, 1598.

Riessner C., Die Magnae Derivationes des Uguccione da Pisa und ihre Bedeutung für die romanische Philologie, Roma 1965.

Rodolico N., Dal Comune alla Signoria. Saggio sul governo di Taddeo Pepoli in Bologna, Bologna 1898 (rist. an. Bologna 1974).

Rolandini Rodulphini Bononiensis, Summa totius artis notariae [...] in eandem summam locupletissimus apparatus qui Aurora per excellentia dicitur [...], Venetiis, apud Iuntas, 1546 (rist. an. Bologna 1977).

Rölker R., Il Registrum privilegiorum del Comune di Modena. Fasi compositive e redazione notarile di un liber iurium del secolo XIII, in AMASLA, s. VIII, VI/I (2003), pp. 55-83.

Rölker R., Nobiltà e Comune a Modena. Potere e amministrazione nei secoli XII e XIII, Modena 1997 (ed. orig. 1994).

Romano G., Un documento cremonese relativo all'Universitas scholarium, in «Archivio Storico Lombardo», s. III, XXIII/2 (1896), pp. 138-149.

Rossetti G., 'Poena' e 'rei persecutio' nell'actio ex lege Aquilia, Napoli 2013.

Rossi G., Historiarum ravennatum libri decem, Venetiis, ex Typographia Guerrae, 1589.

Rossi G., voce Panciroli (Panzirolus) Guido, in DBGI, II, p. 1496-1497.

Roumy F., voce Durand (Durant, Durandi) Guillaume, in Dictionnaire hi- 
storique des juristes français, $X I I^{e}-X X^{e}$ siècle, sous la direction de $\mathrm{P}$. Arabeyre, J.-L. Halpérin, J. Krynen, Paris 2007, p. 290-292.

Rovere A., I «libri iurium» dell'Italia comunale, in Civiltà Comunale: Libro, Scrittura, Documento. Atti del Convegno. Genova, 9-11 novembre 1988, Genova 1989 (=«Atti della Società Ligure di Storia Patria», n.s., XXIX/II), pp. 157-199.

ROVERE A., I «Libri iurium» delle città italiane: problematiche di lettura e di edizione, in «Archivi per la Storia», VI/1-2 (1993), pp. 79-94.

Rovere A., Tipologie documentali nei Libri Iurium dell'Italia comunale, in W. Prevenier, T. de Hemptinne (a cura di), La diplomatique urbaine en Europe au moyen age. Actes du congres de la Commission internationale de diplomatique, Gand 25-29 aout 1998, Leuven 2000, pp. 417-436 (on-line alla URL <http://scrineum.unipv.it/biblioteca/rovere.html>).

RutıLIo B., Iurisconsultorum vitae Bernardino Rutilio auctore, Romae, apud Antonium Bladum, 1536.

Ryan M., Political Thought, in The Cambridge Companion to Roman Law, ed. by D. Johnston, Cambridge 2015, pp. 423-451.

SaвBA F., La 'Bibliotheca universalis' di Conrad Gesner. Monumento della cultura europea, Roma 2012.

Sala E., Della vita e degli scritti di monsignor Giovanbattista Scanaroli da Modena, orazione inaugurale recitata dal professor Erio Sala nella solenne apertura della $R$. Università degli studi in Modena nel giorno 15 novembre 1865, Modena 1866.

Salatiele, Ars notarie, a cura di G. Orlandelli, 2 voll., Milano 1961.

SAndonnini T., Di un codice del XIV secolo e dell'antico Studio modenese, in «Rassegna per la Storia dell'Università di Modena e della cultura superiore modenese», I (1929), pp. 90-129 (ed. orig., priva della revisione del testo e dei regesti finali curati da E.P. Vicini, in AMDSPMo, s. V, IV, 1905).

SANTinI G., Giuristi settentrionali allo Studio di Napoli in età federiciana. Uberto di Bonaccorso e Martino da Fano, in Atti delle seste giornate federiciane (Oria 22-23 ottobre 1983), Bari 1986, pp. 57-71.

SANTINI G., Università e società a Modena tra il XII e il XIII secolo, in Università e società nei secoli XII-XVI. Atti del IX Convegno internazionale di studi, Pistoia, 20-25 settembre 1979, Pistoia 1982, pp. 327-362.

SANTINI G., Università e società nel XII secolo: Pillio da Medicina e lo Studio di Modena, Modena 1979.

SAntoni F., Copisti-editores di manoscritti giuridici: 1. Il codice Vatica- 
no latino 1406 del Digestum Vetus e l'edizione del testo fra copisti e glossatori, in H. SPILLING (a cura di), La collaboration dans la production de l'écrit médiéval. Actes du XIII ${ }^{\mathrm{e}}$ Colloque du Comité international de Paléographie Latine (Weingarten, 22-25 settembre 2000), Paris 2003, pp. 231-249.

SARTI M., De claris Archigymnasii Bononiensis professoribus a saeculo XI usque ad saeculum XIV, tomus I, pars II, Bononiae, Ex Typographia Lelii a Vulpe instituti Scientiarum Impressoris, 1772.

SARTI M.-FATTORINI M., De claris archigymnasii bononiensis professoribus a saeculo XI usque ad saeculum XIV, 2 voll., Bononiae 1888-1896.

Sarti N., Martino da Fano e i suoi «Notabilia super Institutionibus», in RSDI, 69 (1996), pp. 115-154 (riedito con modifiche in EAD., Tre itinerari di storia giuridica: $i$ manoscritti, $i$ giuristi, gli istituti, Torino 2007, pp. 137-170).

SARTi N., Il Liber cautele et doctrine di Uberto da Bobbio, in N. Sarti, S. Bordini, L'avvocato medievale tra mestiere e scienza giuridica. Il Liber cautele et doctrine di Uberto da Bobbio (...1211-1245), Bologna 2011, pp. 99-193.

Sarti N., Martino da Fano e i suoi Notabilia super Institutionibus: una didattica per le università minori, in V. Piergiovanni (a cura di), Medioevo notarile. Martino da Fano e il Formularium super contractibus et libellis, Milano 2007, pp. 135-156.

SArTi N., voce Uberto da Bobbio, in DBGI, II, pp. 1989-1990.

SARTi N., Publicare - exemplare - reficere. Il documento notarile nella teoria e nella prassi del XIII secolo, in G. TAMBA (a cura di), Rolandino e l'ars notaria da Bologna all'Europa. Atti del Convegno internazionale di studi storici sulla figura e l'opera di Rolandino, Bologna - città europea della cultura, 9-10 ottobre 2000, Milano 2002, pp. 613-665.

SARTI N., Il diritto nei fatti. A proposito di una recente pubblicazione di Manlio Bellomo, in RSDI, 84 (2011), pp. 456-469.

Sarti N., La città e lo Studio nell'età di Pietro d'Anzola, in G. TAmba (a cura di), L'opera di Pietro d'Anzola per il notariato di diritto latino. Atti del Convegno di studi storici (Bologna-Anzola dell'Emilia, 6 ottobre 2012), Bologna 2014, pp. 1-9.

SARTI R., voce Fontana Agostino, in DBI 48, Roma 1997, pp. 606-607.

Savigny F.C., Storia del diritto romano nel medio evo, 3 voll., Torino 1854 57 (rist. an. Roma 1972).

Scalvanti O., Notizie e documenti sulla vita di Baldo, Angelo e Pietro degli 
Ubaldi, in L'opera di Baldo per cura dell'Università di Perugia nel V centenario della morte del grande giureconsulto, Perugia 1901, pp. 181-359.

Scanaroli I.B., De visitatione carceratorum libri tres [...], Romae, typis Reuerendae Camerae Apostolicae, 1655.

Schembs H.-O., voce Fichard, in Frankfurter Biographie. Personengeschichtlichen Lexikon, I, Frankfurt am Main 1994, pp. 203-204.

SchIPANI S., Contrbuti romanistici al sistema delle responsabilità extracontrattuali, Torino 2009.

Schlosser H., Prospero Farinacci (1544-1618), ein bedeutender Kanonist?, in «Ins Wasser geworfen und Ozeane durchquert». Festschrift für Knut Wolfgang Nörr, hrsg. von M. Ascheri, F. Ebel, M. Heckel et alii, Köln-Weimar-Wien 2003, pp. 893-903.

Schulte J.F., Die Geschichte der Quellen und Literatur des canonischen Rechts, I-II, Graz 1956 (ed. orig. Stuttgart 1875).

SeEBASS O., Handschriften von Bobbio in der Vatikanischen und Ambrosianischen Bibliothek, in «Centralblatt für Bibliothekswesen», XIII/1 (1896), pp. 1-57.

Sella P., Appunti su alcuni Codici Giuridici Vaticani, in «L'Archiginnasio» XXVIII (1933), pp. 382-84.

Semeraro M., Osberto da Cremona. Un giurista dell'età del dirittto comune, Roma 2000.

Semeraro M., voce Martino del Cassero da Fano, in DBGI, II, pp. 1291 92.

Semeraro M., voce Niccolò Mattarelli (Mattarelli), in DBGI II, pp. 14251426.

Serrai A., Storia della bibliografia, I. Bibliografia e Cabala. Le Enciclopedie rinascimentali (I), a cura di M. Cochetti, Roma 1988.

Serrai A., Storia della bibliografia, II. Le Enciclopedie rinascimentali (II). Bibliografi universali, a cura di M. Cochetti, Roma 1991.

SerRai A., Storia della bibliografia, III. Vicende e ammaestramenti della Historia Literaria, a cura di M. Cochetti, Roma 1991.

Serrai A., Storia della bibliografia, IV. Cataloghi a stampa. Bibliografie teologiche. Bibliografie filosofiche. Antonio Possevino, a cura di M.G. Ceccarelli, Roma 1993.

Sheenan M.M., The Will in Medieval England. From the Conversion of the Anglo-Saxons to the End of the Thirteenth Century, Toronto 1963.

Silingardi G., Catalogus omnium episcoporum Mutinensium [...], Mutinæ, ex officina episcopali apud Ioannem Mariam de Verdis, 1606. 
Simeoni L., Ricerche sulle origini della signoria estense a Modena, in “AMDSPMo", s. V, XII (1919), pp. 127-186.

Simmler J., Epitome Bibliothecae Conradi Gesneri [...], Tiguri, apud Christophorum Froschoverum, 1555.

Simmler J., Appendix Bibliothecae Conradi Gesneri [...], Tiguri, apud Christophorum Froschoverum, 1555.

Simmler J., Bibliotheca instituta et collecta primum a Conrado Gesnero, deinde in Epitomen redacta et novorum librorum accessione locupletata [...], Tiguri, apud Christophorum Froschoverum, 1574.

SinIsi L., Formulari e cultura giuridica notarile nell'età moderna. L'esperienza genovese, Milano 1997.

SinISI L., Alle origini del notariato latino: la Summa Rolandina come modello di formulario notarile, in G. TAмвA (a cura di), Rolandino e l'ars notaria da Bologna all'Europa. Atti del Convegno internazionale di studi storici sulla figura e l'opera di Rolandino, Bologna - città europea della cultura, 9-10 ottobre 2000, Milano 2002, pp. 163-233.

SinIsI L., Nel solco di Rolandino. L'opera di Pietro d'Anzola fra «theorica» $e$ «practica», in G. TАмвА (a cura di), L'opera di Pietro d'Anzola per il notariato di diritto latino. Atti del Convegno di studi storici (BolognaAnzola dell'Emilia, 6 ottobre 2012), Bologna 2014, pp. 45-62.

Sitzia F., Riflessioni in tema di arbitrato in diritto giustinianeo e bizantino, in «Annali del Seminario giuridico dell'Università degli Studi di Palermo», 57 (2014), pp. 239-264.

Soetermeer F., A propos d'une famille de copistes. Quelques remarques sur la librairie à Bologne aux XIII ${ }^{e}$ et XIV ${ }^{e}$ siècles, in SM, s. III, XXX/1 (1989), pp. $425-478$ (riedito in ID., Livres et juristes au Moyen Âge, Goldbach 1999, pp. 95-148).

Soetermeer F., Une catégorie de commentaires peu connue. Les «commenta» ou «lecturae» inédits des précurseurs d'Odofréde, in RIDC 2 (1991), pp. $47-67$ (riedito in ID., Livres et juristes au Moyen Âge, Goldbach 1999, pp. 149-170).

Soetermeer F., Recherches sur Franciscus Accursii. Ses Casus Digesti Novi et sa répétition sur la loi Cum pro eo (C. 7.47.un.), in TR 51 (1983), pp. 3-49 (riedito in ID., Livres et juristes au Moyen Âge, Goldbach 1999, pp. 1-48).

Soetermeer F., Exemplar und Pecia. Zur Hestellung juristischer Bücher in Bologna im 13. und 14. Jahrhundert, in Juristische Buchproduktion im Mittelalter, hrsg. von V. Colli, Frankfurt am Main 2002, pp. 481-516.

Soetermeer F., The origin of ms. d'Ablaing 14 and the Transmissio of the 
Clementines to the Universities, in TR, 54 (1986), pp. 101-112 (riedito in ID., Livres et juristes au Moyen Âge, Goldbach 1999, pp. 83-94). Soetermeer F., Utrumque ius in peciis. Aspetti della produzione libraria a Bologna fra Due e Trecento, Milano 1997.

SofFiETTI I., voce Uberto di Bonaccorso, in DBGI, II, p. 1991.

Soli G., La chiesa di S. Matteo, poi di S. Domenico ed il monastero dei PP. Domenicani, in ID., Chiese di Modena, a cura di G. Bertuzzi, I, Modena 1974, pp. 349-411.

Soli G., La chiesa e il monastero di S. Eufemia, in ID., Chiese di Modena, a cura di G. Bertuzzi, I, Modena 1974, pp. 421-448.

Solmi A., Alberto da Gandino e il diritto statutario nella giurisprudenza del sec. XIII, in «Rivista italiana per le scienze giuridiche», XXXII/I-II (1901), pp. 128-202.

Sorbelli A., Inventari dei manoscritti delle biblioteche d'Italia, 41, Firenze 1930.

Sorbelli T., Emilio Paolo Vicini. Lo storico del Comune di Modena, in AMASLA, s. V, VII (1947), pp. 50-56.

Sorbelli T., In memoria di Emilio Paolo Vicini, in AMDSPMo, s. VIII, I (1948), pp. 15-27.

Sorrenti L., Testimonianze di Giovanni d'Andrea sulle 'Quaestiones' civilistiche, Catania 1980.

SorReNTI L., Tra «lecturae» e «quaestiones» in un esemplare del «codex». Il manoscritto Lucca, Biblioteca Capitolare, 322, in «Quaderni catanesi di Studi Classici e Medievali», 17 (1987), pp. 103-133.

Spagnesi E., Il diritto, in Storia dell'Università di Pisa, 1* - 1343-1737, Pisa 1993, pp. 191-257.

Spagnesi E., voce Denari Odofredo, in DBI, 38, Roma 1990, pp. 700-705.

Spagnesi E., voce Odofredo Denari, in DBGI II, pp. 1450-1452.

SPAGNESI, Introduzione, in Bibliografia delle edizioni giuridiche antiche in lingua italiana, I. Testi statutari e dottrinali dal 1470 al 1700. Bibliografia cronologica, Firenze 1978, pp. VII-L.

Speciale G., Henrigetus magistri Gerardi giudice e cronista. La Marca Trevigiana in un'inedita cronaca trecentesca, in RIDC, 3 (1992), pp. 231-275.

Speciale G., La memoria del diritto comune. Sulle tracce d'uso del Codex di Giustiniano (secoli XII-XV), Roma 1994.

Statuta civitatis Mutine anno 1327 reformata, a cura di C. Campori, Parma 1864 (Monumenti di Storia Patria delle Provincie Modenesi. Serie degli Statuti, XII/1). 
Stephanus Maleretus (o Malleretus), Ingeniosissima lucubratio viri undecumque doctissimi domini Stephani Malereti [...] electionis libere faciende [...], Poitiers, G. Bouchet, 1515 (altra ed.: Lucubratio de electione libere facta et potestate regia, Parisiis, Nicol. Hincman, 1533).

Stintzing (von) R., voce Fichard, Johann von, in Allgemeine Deutsche Biographie, 6, Leipzig 1877, pp. 757-759.

Stintzing (von) R., Geschichte der deutchen Rechtswissenschaft, I, München und Leipzig 1880 (rist. an. Aalen 1957).

Stintzing (von) R.-Oefele (von) E., Freymonius, Johann Wolfgang, in Allgemeine Deutsche Biographie, VII, Leipzig 1877, p. 372.

Storti C., voce Alberico da Rosciate, in DBGI I, pp. 20-23.

Storti Storchi C., Intorno ai Costituti pisani della legge e dell'uso (secolo XII), Napoli 1998.

Storti Storchi C., Prassi dottrina ed esperienza legislativa nell'«opus statutorum» di Alberico da Rosciate, in Confluence de droits savants et des pratiques juridiques. Actes du colloque de Montpellier, 12-14 dec. 1977, Milano 1979, pp. 435-489.

Storti Storchi C., Compromesso e arbitrato nella Summa totius artis notariae di Rolandino, in G. TАмвА (a cura di), Rolandino e l'ars notaria da Bologna all'Europa. Atti del Convegno internazionale di studi storici sulla figura e l'opera di Rolandino, Bologna - città europea della cultura, 9-10 ottobre 2000, Milano 2002, pp. 329-376.

Struve T., Die Salier und das römische Recht. Ansätze zur Entwicklung einer säkularen Herrschaftstheorie in der Zeit des Investiturstreites, Mainz-Stuttgart 1999.

Sünderhauf E.S., Wissenstransfer zwischen Deutschland und Italien am Beispiel des Frankfurter Italienreisenden Johann Fichard (15121581), in Schade K., Rössler D., Schäfer A. (Hrsg.), Zentren und Wirkungsräume der Antikenrezeption. Münster 2007, pp. 99-109.

Sünderhauf E.S., Von der Wahrnehmung zur Beschreibung. Johann Fichards Italia (1536/37), in BöHME H., RAPP CH., RöSLER W. (Hrsg.), Übersetzung und Transformation, Berlin-New York 2007 (Transformationen der Antike, Band 1), pp. 425-453.

Tafaro S., C.I. 7.47.1. Giustiniano e i limiti della condannna del debitore, in S. TAFARO (a cura di), L'usura ieri e oggi, Bari 1997, pp. 213-230.

Tafaro S., Debito e responsabilità. Profili romanistici, Bari 2000.

TAFARo S., La limitazione dei debiti, in «Diritto@Storia», 6 (2007), ed. on- 
line alla URL < http://www.dirittoestoria.it/6/Tradizione-romana/Tafaro-Limitazione-dei-debiti.htm>.

TAISAND P., Les vies des plus celebres jurisconsultes de toutes le nations [...], Paris, L. Sevestre, 1731.

Tamassia N., Baldo studiato nelle sue opere, in L'opera di Baldo per cura dell'Università di Perugia nel $V$ centenario della morte del grande giureconsulto, Perugia 1901, pp. 3-35 (anche in ID., Scritti di storia giuridica pubblicati a cura della Facoltà di Giurisprudenza dell'Università di Padova, II, Padova 1967, pp. 481-502).

ТАмва G., Una corporazione per il potere. Il notariato a Bologna in età comunale, Bologna 1998.

Тамва G., Pietro d'Anzola, il "commentatore" di Rolandino (1257/591312). Appunti per una biografia, in G. TамвA (a cura di), L'opera di Pietro d'Anzola per il notariato di diritto latino. Atti del Convegno di studi storici (Bologna-Anzola dell'Emilia, 6 ottobre 2012), Bologna 2014, pp. 155-195 (già in «Atti e Memorie della Deputazione di storia patria per le province di Romagna», n.s., 61 (2010-11), pp. 161-201).

Tамва G., voce Pietro d'Anzola (a), in DBGI, II, pp. 1580-1581.

Tамва G., voce Pietro d'Anzola (b), in DBI, 83 (2015), pp. 451-453.

TAVILla C.E., Homo alterius: $i$ rapporti di dipendenza personale nella dottrina del Duecento. Il trattato De hominiciis di Martino da Fano, Napoli 1993.

Tavilla E., voce Bellencini (Bellincini) Bartolomeo, in DBGI, I, p. 206.

Tenella Sillani C., L'arbitrato di equità. Modelli, regole, prassi, Milano 2006.

Tilatti A., «Legatus de latere domini pape». Il cardinale Latino e le costituzioni del 1279, in A. Degrand et alii (a cura di), Scritti in onore di Girolamo Arnaldi offerti dalla Scuola nazionale di studi medioevali, Roma 2001, pp. 513-543.

TilatTI A., Legati del papa e propaganda nel Duecento, in La propaganda politica nel basso medioevo, Spoleto 2002, pp. 145-176.

Tiraboschi G., Biblioteca modenese o notizie della vita e delle opere degli scrittori natii degli Stati del Serenissimo Signor Duca di Modena, 6 voll., Modena, presso la Società Tipografica, 1781-86 (rist. an. Sala Bolognese 1970).

Tiraboschi G., Storia della letteratura italiana, 11 voll., Modena 1772-95.

Todeschini G., I mercanti e il tempio. La società cristiana e il circolo virtuoso della ricchezza fra Medioevo ed Età Moderna, Bologna 2002. 
Tomasi F.-Zendri C., Mantova Benavides Marco, in DBI, 69, Roma 2007, pp. 214-220.

Tomasini Iacobi Philippi, Gymnasium Patavinum, Utini, Ex Typographia Nicolai Schiratti, 1654 (rist. an. Sala Bolognese 1986 - Athenaeum. Biblioteca di storia della scuola e delle università, a cura di E. Cortese, D. Maffei, n. 33).

TonIolo F., Frati maestri e libri miniati a Padova tra XIII e XV secolo. La biblioteca dei frati agostiniani del convento degli Eremitani, in Medioevo: $i$ committenti. Atti del XIII convegno internazionale di studi, Parma, 21-26 settembre 2010, a cura di A.C. Quintavalle, Milano 2011, pp. 578-599.

Torelli P.-Vicini E.P., Documenti su Guido da Suzzara, in P. Torelli, Scritti di storia del diritto italiano, Milano 1959, pp. 317-348 (ed. orig. in «Rassegna per la storia della Università di Modena e della cultura superiore modenese», I, 1929).

Tramontana S., La monarchia normanna e sveva, in A. Guillou et alii, Il Mezzogiorno dai Bizantini a Federico II, Torino 1983, pp. 435-768 (Storia d'Italia UTET, III).

Tramontana S., Il Mezzogiorno medievale. Normanni, svevi, angioini, aragonesi nei secoli XI-XV, Roma 2000.

Treggiari F., La laurea del giurista: le orazioni dottorali di Bartolo da Sassoferrato, in A. Esposito, U. Longo (a cura di), Lauree. Università e gradi accademici in Italia nel medioevo e nella prima età moderna, Bologna 2013, pp. 97-111.

Trithemius J., Liber de scriptoribus ecclesiasticis [...], Basileae [Johann Amerbach] 1494 (altra ed. Coloniae, per me Petrum Quentell, 1531).

Trota E., L'ordine dei cavalieri templari a Modena e l'ospitale del ponte di S. Ambrogio, in AMDSPMo, s. XI, VI (1984), pp. 29-55.

Tucc U., Il documento del mercante, in AA.VV., Civiltà comunale. Libro, Scrittura, Documento, Genova 1989, pp. 543-565.

Valentini Forsteri Iurisconsulti, De historia Iuris Civilis Romani libri tres, Basileae, per Ioannem Oporinum et haeredes Ioannis Hervagii, 1565 (confluita poi in TUI, t. I, ff. 25vb-58vb).

VAlENTINI V., Il «Tractatus de tabellionibus» di Baldo degli Ubaldi attribuito anche a Bartolo da Sassoferrato nonché a Gozzadino de' Gozzadini, Milano 1966 (estratto da «Studi Urbinati», 18, 1965-66).

Valentini V., L'ordine degli apparati accursiani in una notizia di Angelo degli Ubaldi, in TR, 53 (1985), pp. 99-134. 
VAlLeRANI M., Il diritto in questione. Forme del dubbio e produzione del diritto nella seconda metà del Duecento, in SM, s. III, 48/I (2007), pp. 1-40.

Vallerani M., Il giudice e le sue fonti. Note su inquisitio $e$ fama nel Tractatus de maleficiis di Alberto da Gandino, in «Rechtsgeschichte. Zeitschrift des Max-Planck-Institut für europäische Rechtsgeschichte», 14 (2009), pp. 40-61.

Vallerani M., Consilia iudicialia. Sapienza giuridica e processo nelle città comunali italiane, in «Mélanges de l’École française de Rome - Moyen Âge», 123/1 (2011), pp. 129-149.

Valsecchi C., Un'auctoritas del primo Trecento. Oldrado da Ponte e i suoi consilia, Milano 2000.

Valsecchi C., voce Oldrado da Ponte (a), in DBGI, I, pp. 1452-1453.

VAlsecCHI C., voce Oldrado da Ponte (b), in DBI, 79, Roma 2013, pp. 191-194.

VALSECCHI C., voce Raimondi Raffaele (Cumano), in DBGI, II, pp. 1649-1650.

van De Wouw H., Zur Textgeschichte des Infortiatum und zu seiner Glossierung durch die frühen Bologneser Glossatoren, in IC, 11 (1984), pp. 231-280.

Vedova G., Biografia degli scrittori padovani, II, Padova 1836.

VedRIANi L., Raccolta de' pittori, scultori, et architetti modonesi più celebri [...], In Modona, per lo Soliani stampator ducale, 1662 (rist. an. Bologna 1970).

Vedriani L., Vite et elogij de' cardinali Modonesi, In Modona, per Bartolomeo Soliani, stampator ducale, 1662.

VedRIANI L., Vita del glorioso s. Geminiano vescouo, e protettore della citta di Modona [...], In Modona, per Andrea Cassiani stampator vescouale, 1663.

VedRIANI L., Memorie di molti santi martiri, confessori, e beati modonesi, e di tutti i corpi santi, che riposano nelle chiesa di Modona [...], In Modona, per Andrea Cassiani stampator ducale, 1663.

Vedriani L., Vita della beata Camilla Pia [...], In Modona, per Andrea Cassiani, 1664.

Vedriani L., Dottori modonesi di teologia, filosofia, legge canonica e civile, in Modona, per Andrea Cassiani stampatore ducale, 1665.

Vedriani L., Historia dell'antichissima città di Modona, 2 voll., Modena, per Bartolomeo Soliani, 1666-67 (rist. an. Bologna 1967).

Vedriani L., Catalogo de vescoui modonesi [...], In Modona, per Bartolomeo Soliani stampator ducale, 1669.

VedRIANI L., Breve racconto dell'arma stilata dalla nobilissima famiglia de Cortesi da Modona [...], In Bologna, per Gioseffo Longhi, 1671. 
Venturi A.R. (a cura di), Girolamo Tiraboschi. Miscellanea di studi, Modena 1997.

Venturi A.R., Girolamo Tiraboschi e le figure dei bibliotecari estensi nella Modena capitale, in A. Spaggiari, G. Trenti (a cura di), Lo Stato di Modena, Una capitale, una dinastia, una civiltà nella storia d'Europa. Atti del convegno, Modena, 25-28 marzo 1998, II, Roma 2001, pp. 1047-1059.

Vermiglioli G.B., Biografia degli scrittori perugini e notizie delle opere loro, t. I, p. I, Perugia, presso Vincenzio Bartelli e Giovanni Costantini, 1828.

Vicini C., La caduta del primo dominio estense a Modena e la nuova costituzione democratica del Comune (1306-1307), Modena 1922.

VIcINI E.P., Di Niccolò Mattarelli ricerche e critica (...1240...1310...), Modena 1900.

Vicini E.P., Di un codice di statuti notarili del secolo XIV, Modena 1910 (estratto da AMASLA, s. III, X/1, pp. 51-112).

Vicini E.P., I Capitani del Popolo di Modena e di Reggio, in «Studi e Documenti della Deputazione di Storia Patria per le Provincie Modenesi», III/IV (1939), pp. 189-209; IV/I (1940), pp. 37-64; IV/III (1940), pp. 171-188; IV/IV (1940), pp. 234-250; V/I (1941), pp. 55-64.

Vicini E.P., I confini della parrocchia del Duomo nel secolo XIV. Saggio di topografia modenese medioevale, in AMDSPMo, s. VII, IV (1927), pp. 65-117.

Vicini E.P., I podestà di Modena (1156-1796). Parte prima (1156-1336), Roma 1913.

Vicini E.P., I Visconti Estensi in Modena, in "Studi e Documenti della R. Deputazione di Storia Patria per l'Emilia e la Romagna. Sez. di Modena”, n.s., I (1942), pp. 24-39.

Vicini E.P., Le pergamene del monastero di S. Eufemia di Modena, in «Studi e Documenti della R. Deputazione di Storia Patria per l'Emilia e la Romagna. Sez. di Modena», I/II (1937), pp. 1-20; I/III (1937), pp. 21-52.

VIcINI E.P., Medici modenesi nei secoli XIII e XIV. Appunti e documenti, Modena 1930 (estratto da «Rassegna per la Storia dell’Università di Modena e della cultura superiore modenese», II, 1930).

Vicini E.P., Profilo storico dell'antico Studio di Modena, Modena 1926.

VICINI E.P., Statuta iudicum et advocatorum collegii civitatis Mutinae MCCLXX-MCCCXXXVII, Modena 1935 (Pubblicazioni della facoltà di Giurisprudenza della R. Università di Modena, 64). 
Vischi L., Sandonnini T., Raselli O. (a cura di), Cronache modenesi di Alessandro Tassoni, di Giovanni da Bazzano e di Bonifazio Morano, Modena 1888 (Monumenti di storia patria delle provincie modenesi. Serie delle cronache 15).

Volante R., voce Bertazzoli Bartolomeo, in DBGI, I, pp. 234-235.

Waelkens L., La théorie de la coutume chez Jacques de Révigny. Edition et analyse de sa répétition sur la loi De quibus (D. 1.3.32), Leiden 1984.

WeIJERs O., Queritur utrum. Recherches sur la 'disputatio' dans les universités médiévales, Turhout 2009.

Widman H., Nachwort, in K. Gesner, Bibliotheca Universalis und Appendix, 2 voll., Osnabrück 1966 (rist. an. dell'ed. Tiguri, apud Christophorum Froschoverum, 1545 e 1555), pp. I-XI.

WIEAcKer F., Privatrechtsgeschichte der Neuzeit, Göttingen $1967^{2}$.

Winterberg H., voce Fichard Johann, in Handwörterbuch zur deutschen Rechtsgeschichte, I, Aachen 1971, pp. 1129-1130.

Woelki T., voce Angelo di Francesco degli Ubaldi (post 1334-1400), in Autographa, I.2., pp. 119-128.

WolfHart C., Elenchus scriptorum omnium, veterum scilicet ac recentiorum [...], Basileae, per Ioannem Oporinum, 1551.

Zaccaria di Martino, Summa artis notarie, a cura di R. Ferrara, S. Giovanni in P. (Bo) 1993.

ZaCCARIA M., Nuovi contributi per la biografia di Bovetino da Mantova ( + 1301), in QSUP, 46 (2013), pp. 281-292.

ZENDri C., voce Mantova Benavides Marco Antonio, in DBGI, II, pp. 12611262.

ZiLETTI G.B., Index librorum omnium nomina complectens in utroque iure tam pontificio quam caesareo, Venetiis, ex officina Stellae Iordani Ziletti, 1559.

Ziliotto P., L'imputazione del danno aquiliano tra iniuria $e$ damnum in corpore datum, Padova 2000. 



\section{INDICE DEI NOMI DI PERSONA}

Si omette la voce Niccolò Mattarelli per l'eccessiva frequenza delle occorrenze. In maiuscoletto i nomi degli autori citati anche nella bibliografia finale. qd.: quondam, v.: vedi

AbBondanZa R., 30

Accorsi, famiglia, 2

Accursio, 1, 20, 24, 40, 76, 81, 150

Accursio da Reggio, 75, 76, 77

Adelardi Guglielmo, 37

Agostino, santo, 149

Agustin A., 163

Al KalaK M., 10

Albergati Nicolò, vescovo, 97

Alberico da Rosciate (A. da RosaTE), 3, 21, 22, 23, 85, 87, 91, 92, 93, 99, 100, 101, 102, 103, 104, 105, 106, 108, 109, 110, 115, 116, 117, 151, 152, 153, 172, 185

Albertina qd. Albertino da Montevallaro, 37

Albertina tezolana, 34

Albertino qd. Martino da Sala, 34

Alberto da Gandino, 23, 104, 106, 108, 109

Alberto de Montis, 49

Alberto di Odofredo, 22, 75, 76

Alberto Galeotti, 27, 76

Alberto Papiense (A. da Pavia), 26, 76
Albertum Bidellum, 71

Albini G., 13

Alciato Andrea, 63, 64, 165, 180

Alessandro d'Este, cardinale, 179

Alessandro III (Rolando Bandinel-

li), papa, 116

Alfonso II d'Este, 182

Altemanno tuscho, 48

Altigrado da Lendinara, 29, 55

Amadeo de Veneciis, 48

Ambrogio da Imola, 48

Amelotti M., 124

Andrea Donolina, 48

Andreolli B., 36, 54, 67

ANDRich G., 145, 156, 190

Angelo da Amelia, 149

Angelo degli Ubaldi, 40, 85, 86, 88, 97, 122, 129, 149, 167

Angiò, v. Roberto, Carlo II, Carlo

Martello, Carlo Roberto, Ludovico

Angustis de, v. Gerardo, Gianbuono, Martino

Anthonio de Rubeis, 49

Anthonius de Aretio, 95 
Antognoli G., 157

AnTONAZZI G., 79

Antonello principe di Salerno, 149

Antonio da Budrio, 115, 116, 155

Antonio de Pizollbechariis, 32

Antonius de Pii, legum doctor, 95

Arabeyre P., 89

Ardizio da Lovoleto, 32

Ardizzone de' Conti, vescovo, 39, 50

Arsendi, v. Federico, Ranieri

AsCheri M., 146, 150, 153, 177, 178

Astegiano L., 26, 29

Augustis de, Gerardo, 35, 51

-, Giambuono, 35, 51

-, Martino, 35, 51

Avesani R., 21

Azzo VIII d'Este, 54, 57, 58

Azzone, 20, 23, 26, 41, 43, 73, 78, $81,86,87$

Baccellieri Niccolò, 127

Baietto L., 13

BALBI G., 103

BALdo degli Ubaldi, 3, 5, 60, 62, 79, 84, 86, 95, 96, 98, 104, 106, 115, 117, 119, 120, 121, 122, 129, 130, 133, 136, 137, 139, 142, 145, 147, 149, 155, 156, 157, 164, 167, 168, 169, 170, 171, 176, 180, 185, 186, 190

Balugola da, famiglia, 50

- Francesco, 50

Baracchi O., 11, 65

BARbiERI A., 182

Barnaba Malaspina, legum professor, 70
Barone dei Mangiatori, podestà, 60

BARTOCCI A., 7

Bartolo da Sassoferrato, 1, 3, 30, $31,62,73,85,103,114,121$, 129, 132, 133, 142, 149, 151, 152, 153, 156, 158, 159, 167, 168, 169, 176, 178, 181, 183, 184, 190

Bartolomeo da Brescia, 83

Bartolomeo, priore, 51

Bartolomeo Spetiali, 54

Bartolus de Corazino, legum doctor, 60

Barufaldino de Lavello, 29

Bassani A., 108

BAUMGÄRTNER I., 85, 119

Bedoni G., 4, 17, 28, 30, 181

Belcaro da Padova, legum professor, 70

Bellincini Bartolomeo, 64

- Francesco, 64, 65

Bellomo M., 1, 2, 5, 20, 24, 25, 28, $29,60,72,73,78,92,93,94$, 95, 96, 97, 98, 99, 100, 101, 102, 105, 106, 109, 110, 111, 112, 113, 114, 115, 156

Belloni A., 145, 147, 156, 158, 188, 190

Belvisi, famiglia, 2

- Jacopo, v. Jacopo Belvisi

Benassati Martino, 39

- Ugolino, 39

Benatti C., 27

Benedetto d'Isernia, 75

Benedetto XII (Jacques Fournier), papa, 174

Bentivoglio Bentivogli/de Bentivoleis, 148, 149

Bentivoglio Giovanni, 73 
Bernardino de Poltoneriis, 17

Bernardino qd. Arduino de Macre-

$$
\text { to, } 35
$$

Bernardus qd. Petri de Suzo, 15, 34

Bernicoli S., 187

Bertazzoli B., 164, 180

Bertazzoli Claudio, 180

Berto de Robertis, 70

BERTONI G., 15, 17

Bertram M., 92

Bertrando del Poggetto, 31, 43, 157

Besta E., 1, 2, 5, 62, 70, 80, 104, $145,155,169$

Bettetini A., 24

Bevilacqua M., 5, 79, 95, 98

Bevilacqua Niccolò, tipografo, 159

Bezemer C.H., 85

Bianchi Lodovico, 68, 183

- Tommasino, 68, 160

Billanovich G., 21, 23

BIROCCHI I., 24, 134, 147

Bitini Giovanni, 97

Boccalini Traiano, 69

BOCCOLARI G., 182

Bochignolli Bernardino, 41, 42

- Giliolo, 41

Bochoni Raynerius, 10

Boconi Giovannibuono, v. Mattarelli Giovannibuono (padre di Niccolò)

Bonaccorso da Sassoferrato, 73

Bonaccorso degli Elisei, 22

BonaCinI P., 12, 14, 17, 37, 43, 45, $47,52,54,127,129$

Bonamici Lazzaro, 166

Bonato E., 70

Bonfigliolo qd. Guidotto da Marano, copista, 44

Bonifacio Morano, notaio, 17
Bonifacio VIII (Benedetto Caetani), papa, 89, 116

Bonincontro archipresbiter, 49

Bonincontro Spingardo, 48

Bonvisi Bonviso, cardinale, 179

BoRDINI S., 26, 27

Borghesani Rainaldo, 35

BorINo I.B., 95, 98

BORTOLAMI S., 58

Boscaino M., 190

Boschetti, famiglia, 52, 58

- Alberto, vescovo, 11, 12

- Dainesio, 52

- Filippo, vescovo, 52

- Nicolò, 52

Bovetino da Mantova, decretorum doctor, 55, 70

Bozzalini Gerardo, 51

BRAidi V., 16, 17, 19, 52, 53, 55, $56,58,61$

Brancati Bartolomeo, 112

BRIANI G., 69

BRINKHUS G., 82, 85

Bruneta Niccolò, notaio, 11, 14, 16, 49

Bruschi U., 8

Buccabadatis de, Raynerius Pance, 47

Buigues O.G., 33

Bulgaro, 77, 79

Burgundio da Pisa, 80

Caccialupi G.B., 145, 146, 147, 148, 149, 150, 154, 167, 171, $172,173,180,190,191$

CAillemer R., 120

Calasso F., 1, 31

Calderini Giovanni, 110, 147, 149

Cammarosano P., 13 
CAmpori C., 10, 50

Canetoli Giovanni, 116

Cani Giovanni Iacopo, 147

Cantarelli Luca, 97

Capestrano da, Giovanni, 171

Cappelli A., 29

CAPucci M., 182

Carletti A. (Angelo da Chivasso), 175

Carlo I d'Angiò, 25

Carlo II d'Angiò, 61, 62

Carlo Martello d'Angiò, 62

Carlo Roberto d'Angiò, 62

Carlo V, re di Francia, 42

Carnevale tezolanus, 34

Carretti, famiglia, 188

Caselli G.C., 84

Castellani Rolando, notaio, 97

Castellano, servitore, 32

Castello da, famiglia, 188

- Rainero, 39

Castiglioni Cristoforo, 88

Cataldino Boncompagni de Visso, 149

Cavalca D., 103, 104, 108

Cavallar O., 103

Cavicchioli Sonia, 179

Cavina M., 8

Cecina A., 46

Cerasi L., 192

Cerroni M., 21

Cesare, 162

Cesarini Sforza W., 127

Cesi Paganino, 48

Chiappini L., 54

Chivasso da, Angelo, v. Carletti A.

Cholet Jean, 42

Ciaralli A., 80

Cicerone, 149
Cino da Pistoia, 3, 7, 22, 23, 31, $62,73,82,83,85,86,87,89$, 90, 95, 99, 100, 106, 114, 117, 121, 122, 125, 126, 132, 140, 142, 151, 159, 172, 183, 186, 189, 190, 192

Cipolla C., 118

Cipriani R., 77

Clemente V (Bertrand de Got), papa, 43, 63

Clemente VIII (Ippolito Aldobrandini), papa, 182

Cochetтi M., 146, 153, 155, 161, 162, 164, 165, 173, 176, 189

Codebò Carlo, 68

Codolis de, Bernardo, 70

-, Stefano de Hemanzo, 70

CoIng H., 1

Colle F.M., 3, 55, 69, 71, 72, 97, 191

Colli G., 164, 165, 176, 177

Colli V., 27, 69, 153, 170, 171

Condorelli O., 89

Conetti M., 21, 23, 93, 104

Conte E., 74

Conversano di Leone da Viterbo, 76

Coppr E., 29, 30

Corbino A., 107

Cornazzano da, Bartolomeo, 76

Cortese E., 6, 25, 73, 86, 87, 88, $89,98,115,116,120,122$, 145, 146, 151, 152, 153, 156, $157,158,170$

Cortesi Oddone, 48

- Niccolò, 48

Coser E., 36

Cosimo I de' Medici, 158

Cosimo Veronesi, 78

Costantino, imperatore, 93 
Costrignano da, Gerardo, 27, 28, Dominicus de Ponte, v. Pontius, 46 magister

Cotta Cattelliano, 145

Donatus de Imola, 79

Cremaschi G., 22, 23

Dondi A., 65

Cremona, da, v. Egidiolo, Nicola, Omobono, Osberto, Uberto

Dufour J., 158

Dumas A., 116

Crescenzi V., 123, 124, 125, 138

Duynstee M., 85, 151

Cursi M.F., 107

D’Amelio G., 103, 145, 149

Dadeo da Cesena, decretorum doctor, 70

DANusso C., 120, 169

Dyonisius de Barigianis, iuris utriusque doctor, 95

De Caro G., 69

DegenRing S., 103

Degoldeo, notaio, 12

Delacore di Pietro da Ganaceto, 40

Delaito di Pietro da Ganaceto, 40

Delayto, notaio, 12

Della Fontana G., 16

Del Negro P., 2, 189, 190, 192

Del Re N., 164

Denari, famiglia, 2

- Odofredo, v. Odofredo Denari

Desanti L., 107

Di Cesare F., 149

Di Noto Marrella S., 146

Di Pietro Paola, 178

Di Pietro Pericle, 4, 26, 27, 28, 40

di Renzo Villata G., 68, 145

Ebel F., 82

Egidia de Poltoneriis, 16, 17

Egidio dei Foscherari, 29

Egidiolo Madalberti (E. da Cremona), decretorum doctor, 30, 63, 70,71

ELZE R., 79

EMILI A., 60

Enselmini Enselmino, giudice, 58

Enrico da Susa (Ostiense), 124

Enrico VII, imperatore, 63

Ercole III d'Este, 64

Eriberto, vescovo, 10

ERrera A., 99

Este, v. Alessandro, Alfonso II, Azzo VIII, Ercole III, Obizzo II

Ezzelino da Este, 59

Facciolati J., 168, 188, 190, 191

FARINACCI P., 164

Dino Rossoni (D. del Mugello), 29, $60,72,75,76,77,82,83,102$, 104, 105, 112, 113, 121, 149

FATTORINI M., 19, 20, 157

FAVINo L., 171

Federico delle Scale, 60, 76

Dionisotti C., 146, 147, 185

Federico di Ranieri Arsendi, 77

Diplovatazio T., 145, 150, 151, 152, 153, 154, 155, 158, 159, 167, 175, 181, 184, 185

Federico II, imperatore, 26, 47

Federicus de Lascha, legum doctor, 60

DolCINI C., 6

Dolezalek G., 76, 79

Federicus de Senis, decretorum doctor, 95 
FeEnStRa R., 85

FEO G., 124

Ferrari M., 118

Ferrara da, Jacopo, vescovo, 181

Ferro del, v. Giovanni, Tommasino

FicHARD J., 162, 164, 165, 166, 167 , 168, 190, 191

Fieschi Sinibaldo, v. Innocenzo IV

FIJAL A., 82

Filipo de Oculis/de Occulis, v. Filippo degli Occhi

Filippo degli Occhi, 48, 49

Filippo II, re di Spagna, 182

FIORI G., 118

Firenze da, Antonino, 175

FitTing H., 73

Floriano di San Pietro, 88

FontANA A., 64, 163, 164, 177, 188

Fontana, famiglia, 184

- Roberto, vescovo, 65, 184

Forciroli F., 64, 178, 179, 180, 181

Foresti G.F. (Filippo da Bergamo), 172, 173, 180, 183, 186

ForNILLI C.C., 181

Forster V., 163

Fortunati M., 136, 139, 140

Fosaregius ferarius, 10

Foschero Pietro, 68

FOWLER L., 33

Francesco d'Accursio, 60, 76, 77, 82

Francesco de Pepis, 88

Francesco de Robertis, 70

Francesco di Grasolfo, notaio e copista, 44

Francesco Guicciardini, 88, 91

Francesco Merzadro, 48

Francesco Petrarca, 21

Franchini V., 127
Francia V., 36

Francesco Albergotti, 95

Fratta dalla, famiglia, 188

FREYMON J.W., 162, 163, 164, 165

FRIED J., 26

FrIEDERICHS H.F., 166

FRIES J.J., 173

Frigerio A., 146

Frugoni C., 15, 65

Fueter E.K., 174

Gabriele Fabricii, 149

Galasso G., 61

Gallieno, imperatore, 79, 140

GALlo D., 192

García y García A., 111

GARDONI G., 55

GaRGAN L., 77, 78

Garsia, canonista, 29

GASSNER B., 176

GAtTI D., 67

GAzZETTI E., 182

Georgis de, famiglia, 118; v. Giorgi

-, Antonio, 118, 119

Gerardino da Bagnolo, 32

Gerardo Carepto/de Careptis, 49, 52

Gerardo de Angustis, 51

Gesner C., 154, 164, 173, 174, 183, 186

GHIRARDACCI C., 30

Ghisalberti C., 104, 106, 108

Giacobbe, 74

Gianbuono de Angustis, 51

GiansANTE M., 36

Giason Del Maino, 150, 159, 168, 183, 186

Giazzi E., 23, 71, 76

Giliolo de' Canonici, 35, 51 
GinANNI P.P., 186, 188

GiordanENGo G., 120, 158

Giordano Caxarius, 51

Giorgi Giannotto, 118, 119

Giovanni Bassiano, 76, 80, 86, 87

Giovanni Crisostomo, santo, 149

Giovanni D'AndRea, 3, 7, 20, 22, 62, 70, 71, 72, 89, 91, 92, 94, 96, 97, 98, 100, 105, 108, 110, 114, 118, 120, 121, 122, 125, 133, 140, 141, 142, 147, 148, 151, 152, 153, 155, 156, 159, 172, 176, 183, 185, 186, 189, 190, 192

Giovanni d'Anguissola, v. Iohannes de Anguisellis

Giovanni da Imola (Giovanni Nicoletti): 72, 88, 114, 115, 133, $134,141,142$

Giovanni da Legnano, 95

Giovanni da Nonantola, 26, 27

Giovanni da Piscarolo, 47

Giovanni da San Martino, 38

Giovanni de Rechenati, 55

Giovanni de Ursinis, canonico, 70

Giovanni de Zochis, 149

Giovanni del Ferro, 48

Giovanni di Dio, 149

Giovanni di Giroldo/Giroldi, 63, 71

Giovanni Fagioli, 75

Giovanni Ispano, 80

Giovannibuono, vicario del Capitolo di Padova, 54

Giovannini C., 65

Giuliano qd. Scaminax, 32

Giustiniano, imperatore, 124, 162

Gloria A., 19, 22, 54, 59, 63, 70, 71, 191, 192

GNAN P., 79
Gómez L., 161, 162, 164

Gonzaga, famiglia, 67

Gorgadelis de, Bartolomea, 31, 74

-, Iacopinus, 31, 74

Gouron A., 158

Grassoni, famiglia, 52, 58

- Bernardino, 46

- Grassone, 46, 47

- Guglielmo, 52

- Rodolfo, 52

Graziadio Andriani, 32, 49

Greci R., 42, 67

Gregorio IX (Ugolino di Anagni), papa, 116

Gregorio X (Tedaldo Visconti), papa, 115,116

Griffolus de Monte Palitiano, legum doctor, 95

Gualandi Giovanni, 40

GualazzInI U., 26, 29

Guecellone da Camino, 76

Guglielmo da Pastrengo, 3, 5, 20, $21,22,23,91,145,172,180$, 185

Guglielmo da Perugia, 149

Guglielmo de Cuneo, 83, 121

Guglielmo de Plaisian, 83

Guglielmo di Laon, 43, 44

Guglielmo Durante, 26, 27, 28, 44, 62, 71, 72, 94, 95, 96, 97, 98, $100,105,108,120,121,124$, $125,134,140,147,152,172$, 176

Guglielmo Oldoini, 47

Guiberto calegarius, 48

Guido a Ferris, doctor decretorum, v. Guido Ferri

Guido da Pavia, 26

Guido da Suzzara, 25, 27, 28, 31, 
46, 75, 76, 77, 80, 81, 82, 83, Iacopo da Foligno, 51 108

Iacopo de Tadulinis, 48

Guido de Husbertis, 46

Iacopo di Grasolfo, 44

Guido di Angelo da Castiglione, 171

IANNACCI L., 124, 125

Guido Ferri, 63, 70, 71

Guidoni, famiglia, 52, 58

Imola da, v. Ambrogio, Donatus, Giovanni

- Francesco, 36, 37

- Gerardo, 36, 37

- Guidoni Giovanna, 52

- Guido, 52

- Pellegrino, 46

Guidotto Arcidiaconi, 47

Guillaume de Cun, v. Guglielmo de Cuneo

Guizzardino da Bologna, 104

Guizzardo da Colorno, 26

HAAN B., 182

HaLm K., 85

HeFele C.-J., 116

Heidemberg Johann, v. Trithemius

Henrigetus, giudice, 76

Houben H., 63

Innocenzo III (Lotario dei conti di Segni), papa, 116

Innocenzo IV (Sinibaldo Fieschi), papa, 12, 116, 121

Innocenzo VIII (Giovan Battista Cybo), papa, 149

Iohannes de Anguisellis, legum doctor, 60

Iohannes Pagliarensis, legum doctor, 95

Iohannis bidellum, 29

Iohannis Ferario de Ganaceto notarius, 49

Irnerio (Guernerius, Hirnerius), 76, 79, 144, 146, 148

Isacco, 74

ISOTTON R., 27

Iacobus Antonius Stenuus, sive de Malitia, 189, 190

Jacques de Révigny, 83, 85, 103

Jean Blanc, 120

Jean de Monchy, 85

Jean Feu, 89

Joannes Igneus, v. Jean Feu

Johannes de Fabrica, 79

Iacopino de Bozalibus, 48

Johannes de Guidonibus de Accu-

$75,76,77,78,82,87$ mulo, 98

Iacopo Belvisi, 82, 83, 102, 106, $112,114,121$

Johannes Jacobus Canis, v. Cani Giovanni Iacopo

IACOPO BOTTRIGARI, 77, 78, 82, 87,

95, 109, 112, 121

Johannes von Heidenberg, 153, 154

JuNG R., 166

IACOPO D'ARENA, 60, 76, 77, 81, 82,

83，90，105，107，108，109， Kantorowicz H., 150

$114,132,147,149$

KARG A., 167 
KirSChNer J., 103

KLeinheyer G., 167

Kocher G., 82

KoHL B.G., 76

KuTtNer S., 79

LABARDI A., 2, 76, 115

Lamandini Tommaso, 54

Latino Malabranca, cardinale, 48, 49,50

LECLERCQ H., 116

LEGENDRE P., 92

LEHMANN K., 82

Lendinara da, v. Altigrado

LePsius S., 158, 167

Levizzani Orazio, 181

Lichanore di S. Lucia, doctor decretorum, 70, 71

LiotTA F., 11, 27

LIPEN M., 177

Lodoyco de Lodoycis, 49

Lovoleto da, v. Ardizio

LORENZONI G., 46

Loschiavo L., 27, 30

Lovato da, Lovato, giudice e podestà, 70,76

-, Rolandino, 70

Luca da Penne, 149

LuCCHESI C., 97

LuCCHI G., 12

Ludovico d'Angiò, 62

Ludovico il Bavaro, imperatore, 174

Lugardo Enrico, 146

Maccagnano degli Azzoguidi, 78

Macino F., 120, 121, 185

MAFFEI D., 22, 30, 60, 83, 92, 93, $96,97,98,145,146,155,171$
Maffei P., 7, 90, 147

Maire Vigueur J.-C., 49

Malagola C., 19

Malcapelli de', Leone, 70

Malleret Etienne (Stephanus Maleretus/Malleretus), 177

Mancini Giorgia, 179

Manicardi A., 11

Mansueto da Perugia, 149

Mantovani G.P., 73

Marangon P., 59, 71, 72, 74

Marcello A., 90

MarchetTI P., 128

Marco Mantova Benavides, 154, 155, 156, 157, 158, 159, 165, 166, 173, 184, 186, 190, 191

Marcus de Perusio, 95

MARI M., 179

MARI P., 119

Mariconda Diomede, 146

Marrara D., 158

Marrella F., 33

Martino, 80

Martino del Cassero (M. da Fano) $11,24,26,75,76,77,81,82$, 83

Martino de Angustis, 51

Martino F., 25, 69, 83, 84, 99

Martino Garato, 85, 119

Martino Sillimani, 76, 106, 110

Martinoli L., 110, 112, 113

Martone L., 33

Matharel Antoine (Antonius Matharellus), 177

Mattarelli, famiglia, 188

- Achille, 188

- Antonio, 2, 16, 54, 56, 63

- Attaleus/Athaleus, 18, 54, 56

- Bartolomea, 54, 61 
- Bartolomeo, 54, 61, 63

- Bitina, 39

- Corradino/Corrado, 18, 56

- Diocles/Dyocles, 16, 18, 56, 71

- Diomedes/Diomede, 16

- Francesco, 2, 16, 18, 31, 32, 35, $39,50,51,52,54,56,59,61$, $63,71,72,74,82,90,110$, 151, 152, 157, 187, 188, 189, 191

- Gentilis/Gentile, 16, 39

- Gian Novello, 15, 16, 17, 18, 32, $34,36,37,38,39,50,51,54$, 56,63

- Giovanni, 187

- Giovanni Battista, 188

- Giovannibuono (figlio di Niccolò), 2, 53, 56, 61, 63

- Giovannibuono (padre di Niccolò), $2,10,11,12,14,15,18$, $34,37,38$

- Manfredino, 61

- Richelda, 10, 53, 61

- Sander/Alessandro, 15, 16, 17, $18,34,37,38,50,51,53,54$, $56,57,71$

- Thomas/Tommaso, 16

- Ubertino, 54, 61, 63

Matteo, santo, 149

Matteo Gribaldi Mofa, 146

Matteo Mattesillani, 116

Mattone A., 4, 147, 159, 161

MAZZACANE A., 150, 164

MazzANTI G., 25, 27, 29, 97

Meccarelli M., 33

Medica M., 41, 42

Medici, v. Cosimo I

Megli Frattini L., 173

Meijers E.M., 79, 89, 168
Mellusi G.G., 60

Menant F., 47

Mentzel-Reuters A., 82, 85

MenZinger S., 41, 74, 76

Mercati A., 97, 118

Mercati S.G., 103

Merzbacher F., 116, 162

Meyer G., 85, 126, 129, 130

Michele Paleologo, imperatore, 46

Migliarini de', Armanino, 46

-, Corradino, 27, 32, 39, 46, 49

Migliorino F., 92, 110

Milani G., 47, 103

Missere Fontana F., 159

Modenese Purzilini, 48

Modesti M., 125

Molza Gerardino, 53, 54, 61

- Giunta, 53

- Guido, 53, 61

Mommsen Th., 5

Montanos Ferrin E., 102

Montfaucon De, B., 119, 186, 187

Montorzi M., 68, 156, 169

Mor C.G., 4, 26, 27, 28, 40, 80

Morelli G., 134

Mozzato A., 33

MÜLLER W.P., 80, 119

Munari Armanino, 46

- Gerardino, 46

Murano G., 2, 7, 25, 28, 29, 35, 37, $38,40,41,42,44,68,71,85$, 119, 134, 135, 145, 146, 167, 170

MURATORI L.A., 153, 184, 186

Mutinensis fratris Valentini, notaio, 32

MuzZarelli M.G., 146

NajEMy J.M., 49 
NARdi P., 11, 145

Narducci E., 149

Nasi Leonardo, 158

Nello da S. Gemignano, 108

Nevizzano Giovanni, 161, 162, 164

Nicholao de Rebusatis, 49

Nicola da Cremona, 24, 95, 99

Nicolaj G., 79, 119

Nicolaus Michaelis, 116

Nicolaus Plebeius, 189, 190

Nicolini U., 1, 83

Nicolò Bruneta/de Brunecta, notaio

Nicolò Furioso, 80

Nicolò Spinelli, 78, 79

Nicolò II (Gerard de Bourgogne), papa, 62

Nicolò III (Giovanni Gaetano Orsini), papa, 48

Nicot Jean, 151

Nonantola da, v. Giovanni, Petrus

Nuovo A., 171

Obizzo II d'Este, 51, 54

Obizzo, giudice, 60

Oddone, 83

Odofredo Denari, 5, 20, 21, 22, 23 , $24,25,75,77,80,82,83,86$, $87,126,132,145,146,186$

Odorico Trenti, 48

Oefele E. von, 162

Oldrado da Lodi (O. da Ponte), 20, 22, 59, 63, 70, 71, 77, 91, 112, 146, 149, 152, 170, 172, 184

Omobono Morisio (O. da Cremona), 26, 27, 29, 76, 120

Opizzonibus de, Bernardinus, 47

Oprandino de Solaria, 46

Orlandelli G., 127, 134
Osberto da Cremona, 30, 31

OsLer D., 160

Ottone da Pavia, 79

Pace de Pacibus, legum doctor, 59

Pace de Tadis, legum doctor, 59

Pace G., 77, 78, 79

Padoa Schioppa A., 11

Padovani A., 6, 8, 24, 29, 31, 72, 73, 78, 88, 99, 115, 116, 133, 169, 173, 175

Pagliaroli Matteo, 179

Palmerio di Martino da Fano, 11

Palmieri G.B., 104

Panciroli G., 65, 68, 145, 146, 147, 158, 159, 160, 167, 175, 183, $184,189,190$

Panini F., 160, 188

Paolo de Sulimano, doctor legum, 63, 71

Paolo de Titulo, giudice, 59

Paolo di Castro, 116, 142, 167, 176

Paolo III (Alessandro Farnese), papa, 64

Paolo V (Camillo Borghese), papa, 118

Paolo, santo, 149

Papadopoli N.C., 189, 190

PAPIAS, 103

Papillon Ph., 160

Parmeggiani R., 8, 97

Pascipovero, 24

Pasolini S., 187, 188

Pasqua scudario, 49

Passaponti Guido, 32

- Iacopo, 32

- Maria, 32

Patusio Giovanni Battista, 156 
Paolo de Titulus/Paulus Titulus, 59, Pietro da Montecucco, 38, 39, 53 189, 190

Pietro de Podiodorphila, 83

PaZZaglini P.R., 103

Pecorella C., 120, 122, 126

Pietro de Suntino, 149

Pietro dei Cerniti, 75, 112

Pepo, 6

Pepoli Taddeo, 157

Peruzzi P., 113, 119, 167

Pescani P., 80

Pescatore G., 147, 150

Petitjean M., 160

Petitmengin P., 187

Petoletti M., 23

Petrezzani Guglielmo, 51

- Siletto, 48

Petrucci Giovanni, 88

Petrus (da Nonantola), 32

Petrus Benintendus, 91

Petrus de Bellapertica, v. Pierre de

Belleperche

Petrus de Lusscho, 42

Petrus de Suzo, 15, 34

Petrus Roberti, 46

Petti Balbi G., 11, 118

Pezzella S., 175

Pietro del Bosco, notaio, 42

Pietro preceptor et procurator, v. Pietro da Montecucco

Pietro qd. Antonio da Roteglia, 36

Pietrobono qd. Ianuario, 48

Pillio da Medicina, 25, 28, 104, 120

Pincella S., 12

Pio, famiglia, 54

- Egidio, 54, 61

- Manfredino, 54, 61

Platon Bernardo, vescovo, 72

Polenta da, Lamberto, podestà, 187

Polizzi F., 21

Poltoneriis, v. Bernardino, Egidia

Pomaro G., 64, 68

Pomponio, 149

Pontius, magister, v. Ponzio

Ponzio, 149

Porcellini Antonio, 145, 156, 190

Portenari A., 183, 185

Philippus domini Petri de Rodelia, Possevino Antonio, 159 46

Post G., 29

Preto P., 191

Prosdocimi L., 22, 23, 64

PiAna C., 72

Piano Mortari V., 33

Piccialuti M., 145

Piergiovanni V., 33, 139

Pieri B., 8

Pierre de Belleperche, 64, 83, 85, $86,88,89,91$

Pietro Bernardo de Cedollis, 44

Pietro d'Ancarano, 97, 149

Pietro d'Anzola, 135

Puncuh D., 13

Quaglioni D., 23, 73, 76, 90, 98, 99, 106, 108, 117, 145, 146, 156

Rabotti Giuseppe, 150

RADDING C.M., 80

Raffaele Fulgosio, 77, 78, 119, 156

Pietro da Ganaceto 40; v. Delacore, Delaito

Raffaele Raimondi (Cumano), 156

RAGGI O., 181 
Rainerius de Monterubiano, legum RossetTI G., 107 doctor, 95

Rossi GIOVAnNI, 159

Rangoni, famiglia, 52, 58

Rossi Girolamo, 188

- Alda, 52

- Erminia, 52

Rossi Giovanni Antonio, 166

- Gerardino, 32

Roumy F., 28

- Iacopino, 32

ROVERE A., 13

- Tobia, 32, 52

Ruini Carlo, 88, 90

Rutilio B., 165

Ranieri Arsendi (R. da Forlì), 30, Ryan M., 85 $72,77,121,158$

Ranieri da Perugia, 124, 135

SABBA F., 164, 174

RASELLI O., 67

Restani Giovanni, 32

Sadoleto, famiglia, 65, 184, 188

- Giovanni, 88

Riccardo Malombra, 2, 22, 55, 59, - Jacopo, 65 $62,63,70,71,76,82,112$, 152

Ricci D., 181

RicCIARDI R., 147

Riccoboni A., 184, 189

Richelda qd. Raynerii Bochoni, 10

Ricobono de' Tregesi, 48

RIESSNER C., 103

Riticheldi Alberto, 32

- Giovanni, 32

- Iacopo, 32

- Simone, 32

Robertis de, v. Berto, Francesco

Roberto d'Angiò, 61, 62, 63

Roberto il Guiscardo, 62

Rodolico N., 157

Roffredo Beneventano, 75, 79

Rogerio, 80

- Nicolò, 65

Sala E., 181

SAlatiele, 24, 126, 130

Saliceto da, famiglia, 2

-, Bartolomeo, 78, 79, 82, 83

-, Riccardo, 77, 78, 95

Sambiagio da, Giovanni Battista, 145,156

Sandei Felino, 64, 68

SANDONNINI T., 40, 41, 42, 43, 44, 67

SANTINI G., 10, 17, 26, 27, 45, 50

SANTONI F., 79

SARTI M., 19, 20, 157

SARTI N., 1, 5, 8, 24, 26, 127, 135

SARTI R., 164

Sassuolo da, famiglia, 52, 58

Savignano da, famiglia, 52, 58

Rolandino Passaggeri, 135, 136, -, Corradino, 52 147

Rolandino, campanario, 48

Rolando di Petrus de Lusscho, 42

RÖLKER R., 12, 26, 34, 37, 45, 50

SAVIGNY F.C., 3, 20, 22, 28, 31, 145, $160,167,174,192$

Scaligeri, famiglia, 67

SCALVANTI O., 170

Romano A., 92, 100, 101, 115, 152

RomANo G., 29

Scanaroli G.B., 178, 179, 181, 183

Scardeoni Bernardino, 189 
SchembS H.-O., 167

SCHIPANI S., 107

Schlosser H., 164

SCHRÖDER J., 167

Schulte J.F., 83, 175

Schulz F., 150

Scodobi B., 63, 64, 65, 180, 183

Scudari Enrico, 53

- Modenese, 38, 53

- Tomasina, 38, 53

SeEbas O., 118

Sella P., 79, 119

Semeraro M., 2, 11, 27, 30, 31

Seneca, 118

Serrai A., 21, 144, 146, 154, 159, 161,162, 164, 172, 173, 176, 177, 182

SheEHAn M.M., 116

Signorolo da Milano, 149

Silingardi G., 181, 182, 193

SiMEONI L., 45

SIMMLER J., 173

Simone, abate, 49

Simone de Carnelvariis, 32

Simone Vicentino, 76, 77

SINISI L., 134, 135, 167

Sisto IV (Francesco della Rovere), papa, 64

SitZiA F., 139

Socini/Sozzini, famiglia, 2

- Bartolomeo, 88, 90, 166

- Mariano, 166

Soetermeer F., 22, 40, 41, 42, 43, $44,89,90$

SOFFIETTI I., 26

Soli G., 10, 65

Solmi A., 3, 23, 92

Sorbelli A., 95

Sorbelli T., 4
SorrentI L., 26, 69, 72, 94, 95, 96, 97, 98, 100, 105, 185

Spadari Bartolomeo, notaio, 14

SPAGNESi E., 24, 158, 161

Speciale G., 25, 76, 77, 78, 79, 82

Speciali Francesco, 51

Spino de Nebola, 48

Stefanino da Bagnolo, 32

StintZing R. von, 162, 165, 166

Storti Storchi C., 23, 33, 102, 117 , 158

Strabone, 149

Struve T., 6

SÜNDERHAUF E.S., 166

Taddeo de Pocaterris, 55

TAFARO S., 88

TAISAND P., 160

TAMASSIA N., 169

ТАмва G., 124, 126, 127, 129, 130, 135

Tancredi, 83

Tassoni Alessandro, 67

TAvilla E., 8, 11, 16, 19, 26, 27, 52, 56,64

Tebaldi, famiglia, 188

Tedelendi Gianbuono, 34

- Guglielmo, 34

- Iacopino, 34

- Sigizo, 34

Tenella Sillani C., 33

Tigrini Francesco, 88, 95, 119, 167, 183

Tilatti A., 49

Tiraboschi G., 3, 8, 26, 57, 64, 178, $184,185,186$

Todeschini G., 117

TOMASI F., 154

ToMAsinI G.F., 189 
Tomasino de Cazadraghis, legum professor, 70

Tomax de la Fossa, 157

Tome Guglielmo de Hemanzo, 70

- Pietro, 70

Tommasino del Ferro, 53

Tommaso di Piperata, 112

Tommaso Pizoni, 48

Tommaso/Tommasino da Spezzano, 27,46

Toniolo F., 79

ToReLli P., 27, 128

Torti Torberto, 119

Tramontana S., 61, 63

TRegGiari F., 73

TRITHEMIUS J., 173, 174, 175, 180, 186

Trota E., 38

TuccI U., 136

Ubaldi, famiglia, 2

Uberto da Bobbio, 26, 76, 81, 83, 120, 132

Uberto da Cremona, 112

Uberto di Bonaccorso, 26, 83, 120

Ugolino de Ançillino, 49

Ugolino de' Crespi, 35

Ugolino Presbiteri, 25, 75, 76

Ugolino qui non sudat, 48

Uguccione da Pisa, 103

Ungarini Tomasino, 36, 51

Ulpiano, 82

VALENTini V., 40, 121

VALLERANI M., 41, 73, 99, 109, 110, 113

VALSECCHI C., 22, 156, 170

van DE Wouw H., 43
Varnerius de Falchis, 76

Vedova G., 189, 191

VEDRIANI L., 64, 65, 153, 178, 179, $182,183,184,185,188,190$

VentuRi A.R., 179, 186

Vermiglioli G.B., 168

Vianisio dei Passipoveri, 112

VICINI E.P., 2, 4, 10, 11, 15, 16, 17, $18,26,27,28,38,40,42,44$, $45,46,48,52,53,55,56,59$, 63, 71, 157, 187, 192

Vincenzo de Paliottis, 88

VISCHI L., 67

VOLANTE R., 180

WaeLKens L., 85, 86, 87

Weigand R., 80

WeIJers O., 96, 110

WidMAN H., 154, 174

WIEACKER F., 167

WinTERBERG H., 166, 167

Woelki T., 86

Wolfhart C., 173

Zabarella Francesco, 97

ZaCCARIA DI MARTINO, 135

ZACCARIA M., 55

Zanilino becarius, 51

Zanino da Bagnolo, 32

Zanne de Sigonibus, notaio, 17

Zanne qd. Ugolino, 31, 74

Zasius Ulrich, 166, 167

ZENDRI C., 154

ZiletTI G.B., 64, 159, 163, 164, 167

ZiLiotTo P., 107

Zuccardi Ubertino, 155

Zuffrano A., 124, 125

Zunta de Moza, 48 

INDICE DEI NOMI GEOGRAFICI

Abruzzo, 149

Accumuli (RI), 98

Alessandria, 166

Amelia (TR), 149

Arezzo, 11, 60, 95

Avignone, 21, 63, 71

Bagnolo (RE), 32

Balugola (MO), 50

Basilea, 153, 163, 164, 165, 166, 174

Beauvais, 42

Berlino, 6

Bobbio (PC), 118

Bologna, 11, 19, 24, 25, 28, 29, 30, $31,40,41,42,43,45,49,55$, $58,59,63,64,70,73,77,78$, $80,82,89,92,97,108,110$, $112,127,128,129,133,141$, 155, 156, 157, 158, 159, 178, 183, 184, 189, 190

Bomporto (MO), 34

Borgo San Sepolcro (AR), 70

Borgogna, 160
Calabria, 61, 63

Campi Flegrei, 166

Carpi (MO), 54

Cesena, 55, 60, 70, 91

Cittanova (MO), 49

Cittanova d'Istria, 189

Corlo (MO), 36

Corneto (VT), 98

Correggio (RE), 155

Costantinopoli, 130

Cremona, 1, 2, 4, 7, 29, 30, 46, 47, $63,70,71,76$

Digione, 160

Felina (RE), 68

Ferrara, 52, 64, 82, 103, 133, 149, $150,155,180$

Finale (MO), 181

Firenze, 48, 49

Foligno (PG), 4, 7, 36, 51, 61, 70

Forlì, 30

Francia, 42, 82, 160, 182

Francoforte, 156, 162, 165, 166, 167 
Freiburg, 166

Freto (MO), 35

Ganaceto (MO), 40, 49

Genova, 11, 27, 64

Girona, 42

Gorzano (MO), 34

Heidelberg, 166

Imola (BO), 78, 79

Italia, 49, 63, 85, 103, 165, 166, 177,184

Laon, 43

Lione, 116, 156, 161, 165

Lombardia, 6

Losanna, 63, 71

Lucca, 46, 65, 68, 159, 160, 182, 183,187

Macerata, 181

Magreta/Macreto (MO), 35

Mantova, 25, 27, 54, 63, 70, 71

Marano (MO), 44

Marche, 49

Massa (MO), 32

Melfi (PZ), 62

Modena, 2, 4, 7, 9, 10, 11, 12, 14, $15,16,17,22,24,25,26,27$, 28, 29, 32, 34, 36, 37, 38, 39, 40, 41, 42, 44, 45, 46, 47, 48, $49,50,51,52,53,54,55,57$, $58,60,61,63,64,65,67,69$, $70,80,81,82,88,91,94,120$, 127, 151, 156, 159, 172, 178, 180, 181, 182, 183, 184, 185, 186, 191

Monte Palitiano, 95
Monterubbiano (Fermo), 95

Montesperello (PG), 88

Montevallaro (MO), 37

Napoli, 25, 61, 63, 75, 77, 113, 150, 164, 166

Navicello (MO), 35

Nola (NA), 149

Nonantola (MO), 32, 34, 35, 48, 51

Norcia (PG), 98

Orléans, 42, 85, 151

Oxford, 97

Padova, 1, 2, 5, 7, 22, 25, 50, 54, 55, 57, 58, 59, 60, 61, 63, 70, 71, 76, 77, 78, 79, 80, 81, 82, 94, 97, 102, 108, 111, 112, 113, 121, 133, 145, 149, 151, 152, 155, 156, 159, 165, 166, 170, 171, 178, 181, 182, 183, 184, 185, 186, 189, 190, 191

Panzano (MO), 34

Parigi, 42, 160

Parma, 26, 46, 64

Pavia, 73, 78, 89, 119, 156, 165, 170,171

Perugia, 30, 31, 46, 84, 98, 113, 121, 145, 146, 149, 150, 167, 170

Pisa, 31, 88, 116, 155, 156, 157, 158, 159, 183, 184, 185, 190

Prataglia (AR), 79

Prussia, 6

Puglia, 63

Ravenna, 2, 187, 188

Reggio E., 11, 29, 81, 82, 91, 97, 180

Reims, 43 
Ripatransone (AP), 182

Sponheim, 153

Roma, 64, 89, 113, 145, 146, 148, Stoccarda, 85 $149,165,166,179$

Romagna, 49

Tolosa, 78

Roteglia (RE), 36, 46

Torino, 118, 159

Toscana, 49

Saint-Lucien de Beauvais, 42

Trebbio (MO) 37

Sala (BO), 34

Treviso, 2, 187

Salerno, 149, 150

San Felice (MO), 46

Trino (VC), 156, 171

Trittenheim, 153

San Marco (BO), 14

Tubinga, 82, 85

San Miniato (PI), 60

San Salvatore (MO), 35

Urbino, 64, 149

San Severino Marche (MC), 145, 149

Venezia, 21, 25, 63, 146,156, 164,

Santa Vittoria in Matenano (Fermo), 149 171,192

Vercelli, 26, 30

Verona, 21, 171

Vienna, 6

Vignola (MO), 15, 32, 34

Villavara (MO), 34, 35, 51

Sicilia, 63

Viterbo, 76, 98

Siena, 88, 95, 103, 106, 133, 145, 149

Volterra (PI), 46, 47

Solara (MO), 46

Spagna, 182

Würzburg, 153

Spezzano (MO), 27, 46, 188

Spira, 165

Zurigo, 164, 173 



\section{SOMMARIO}

\section{CAPITOLO I CLARUS JURIS CAESAREI PROFESSOR \\ TAPPE DI UNA BIOGRAFIA PROFESSIONALE E POLITICA}

1. Le origini famigliari

2. La matricola dei giudici e avvocati e la matricola dei notai $\quad 15$

3. La formazione giuridica: verso una 'linea alternativa' 18

4. Lo Studio modenese nel Duecento e il primo magistero di Mattarelli 25

5. Denaro, case, terre 31

6. La committenza di manoscritti 39

7. Al governo del Comune 44

8. Ancora nelle file del Popolo 54

9. Il ritorno a Padova 59

CAPITOLO II

FONS BONA, NIHIL ALIUD

OPERE DOTTRINARIE E LORO RICEZIONE NELLA SCIENZA GIURIDICA

1. Nessuno è profeta in patria 67

2. Accipe igitur librum sacratissimarum legum 70

3. Tracce sparse di attività didattica $\quad 75$

4. Repetitiones e tractatus 83

5. Nello strumentario del giurista postaccursiano: le quaestiones 91

6. Libri magni quaestionum 109

7. Un isolato interesse canonistico 115

8. De hac materia summam prolixam formavit 118

8.1. Le imbreviature dei notai 123

8.2. Le scritture dei mercanti 136 


\section{CAPITOLO III \\ SCIENTIA CLARUS, SED ELOQUIO RUDIS \\ LA MEMORIA DI MATTARELLI NELLA LETTERATURA STORICO-GIURIDICA}

1. Storia e biografia giuridica tra Quattro e Cinquecento 143

2. Da Caccialupi a Panciroli $\quad 145$

3. Un genere nuovo: la bibliografia giuridica 160

4. Altri percorsi della bibliografia germanica 165

5. Esempi di bibliografie universali e settoriali 171

6. Erudizione locale fra Cinque e Settecento 178

$\begin{array}{ll}\text { 7. Glorie patrie non soltanto modenesi } & 187\end{array}$

Manoscritti e documenti d'archivio 193

Indice dei passi del Corpus Iuris Civilis e del Corpus Iuris Canonici 197

$\begin{array}{ll}\text { BIBLIOGRAFIA } & 199\end{array}$

$\begin{array}{ll}\text { INDICE DEI NOMI DI PERSONA } & 245\end{array}$

$\begin{array}{ll}\text { INDICE DEI NOMI GEOGRAFICI } & 261\end{array}$ 


\section{P U B B L I C A Z I O N I D E L S E M I N A R I O G I U R I D I C O DELLA UNIVERSITÀ DI BOLOGNA}

1. Coli U., Collegia et sodalitates, 1913.

2. Donatelli I., La "consortia" di Avesa, 1914.

3. VAlenZa P., Il diritto di usufrutto nelle leggi sulle tasse del registro, 1915.

4. Zingali G., La statistica della criminalità, 1916.

5. Tumedei C., La separazione dei beni ereditari, 1917.

6. Albertoni A., L“Apokeryxis", 1923.

7. SALVI F., La cessione dei beni ai creditori, 1947.

8. Milani F., Distinzioni delle servitù prediali, 1948.

9. FAssò G., I "quattro autori" del Vico, 1949.

10. FERri L., La trascrizione degli acquisti "mortis causa" e problemi connessi, 1951.

11. Rossi G., La "Summa arboris actionum" di Ponzio da Ylerda, 1951.

12. Poggeschi R., Le associazioni e gli altri gruppi con autonomia patrimoniale nel processo, 1951.

13. Matteucci N., Antonio Gramsci e la filosofia della prassi, 1951.

14. Forchieldi P., I contratti reali, 1952.

15. SALvi F., Il possesso di stato familiare, 1952.

16. FASsò G., La storia come esperienza giuridica, 1953.

17. Palazzini Finetti L., Storia della ricerca delle interpolazioni nel Corpus iuris giustinianeo, 1953.

18. Rossi G., Consilium sapientis iudiciale, 1958.

19. Mancini G.F., La responsabilità contrattuale del prestatore di lavoro, 1957.

20. FERRI L., L'autonomia privata, 1959.

21. Torelli P., Scritti di storia del diritto italiano, 1959

22. Santini G., I Comuni di Valle del medioevo. La Costituzione federale del "Frignano", 1960.

23. GIANNITI F., I reati della stessa indole, 1959.

24. GHEZZI G., La prestazione di lavoro nella comunità familiare, 1960.

25. NARDI E., Case "infestate da spiriti" e diritto romano e moderno, 1960.

26. FERRI L., Rinunzia e rifiuto nel diritto privato, 1960.

27. GHezzi G., La responsabilità contrattuale delle associazioni sindacali, 1963.

28. Bonsignori A., Espropriazione della quota di società a responsabilità limitata, 1961.

29. Redenti E., Scritti e discorsi giuridici di un mezzo secolo, vol. I, Intorno al diritto processuale, 1962.

30. REDENTI E., Scritti e discorsi giuridici di un mezzo secolo, vol. II, Intorno al diritto sostanziale, 1962.

31. Gualandi A., Spese e danni nel processo civile, 1962.

32. Bonsignori A., Assegnazione forzata e distribuzione del ricavato, 1960.

33. Mancini G.F., Il recesso unilaterale e $i$ rapporti di lavoro, vol. I, Individuazione della fattispecie. Il recesso ordinario, 1962.

34. NARDI E., Rabelais e il diritto romano, 1962.

35. Romagnoli U., Il contratto collettivo di impresa, 1963.

36. Santini G., I "comuni di pieve" nel medioevo italiano, 1964.

37. RUdAN M., Il contratto di tirocinio, 1966.

38. Bonini R., I "libri de cognitionibus" di Callistrato. Ricerche sull'elaborazione giurisprudenziale della "cognitio extra ordinem", 1964.

39. Colliva P., Ricerche sul principio di legalità nell'amministrazione del Regno di Sicilia al tempo di Federico II, 1964.

40. Mengozzi P., L'agenzia di approvvigionamento dell'Euratom, 1964.

41. Scritti minori di Antonio Cicu, tomi I e II, Scritti di teoria generale del diritto - Diritto di famiglia, 1965.

42. Scritti minori di Antonio Cicu, Successioni e donazioni. Studi vari, 1965.

43. SACChi Morsiani G., Il potere amministrativo delle Comunità europee e le posizioni giuridiche dei privati, I, 1965.

44. GHEZZI G., La mora del creditore nel rapporto di lavoro, 1965.

45. Roversi Monaco F.A., Enti di gestione. Struttura, funzioni, limiti, 1967.

46. Gianniti F., L'oggetto materiale del reato, 1966. 
47. Mengozzi P., L'efficacia in Italia di atti stranieri di potestà pubblica su beni privati, 1967.

48. Romagnoli U., La prestazione di lavoro nel contratto di società, 1967.

49. Montuschi L., I limiti legali nella conclusione del contratto di lavoro, 1967.

50. Ranieri S., Scritti e discorsi vari, vol. I, Scritti di diritto penale, 1968.

51. Ranieri S., Scritti e discorsi vari, vol. II, Scritti di procedura penale, 1968.

52. Bonini R., Ricerche di diritto giustinianeo, 1968.

53. Santini G., Ricerche sulle "Exceptiones legum romanorum", 1969.

54. Lo Castro G., La qualificazione giuridica delle deliberazioni conciliari delle fonti del diritto canonico, 1970.

55. Sacchi Morsiani G., Il potere amministrativo delle Comunità europee e le posizioni giuridiche dei privati, II, 1970.

56. Roversi Monaco F.A., La delegazione amministrativa nel quadro dell'ordinamento regionale, 1970.

57. Gianniti F., Studi sulla corruzione del pubblico ufficiale, 1970.

58. De Vergottini G., Indirizzo politico della difesa e sistema costituzionale, 1971.

59. Mengozzi P., Il regime giuridico internazionale del fondo marino, 1971.

60. CARINCI F., Il conflitto collettivo nella giurisprudenza costituzionale, 1971.

61. Osti G., Scritti giuridici, voll. I e II, 1973.

62. Zuelli F., Servizi pubblici e attività imprenditoriale, 1973.

63. Pergolesi F., Sistema delle fonti normative, 1973.

64. Montuschi L., Potere disciplinare e rapporto di lavoro, 1973.

65. Pattaro E., Il pensiero giuridico di L.A. Muratori tra metodologia e politica, 1974.

66. Pini G., Arbitrato e lavori pubblici, 1974.

67. CARPI F., L'efficacia "ultra partes" della sentenza civile, 1974.

68. De Vergottini G., Lo "Shadow cabinet", 1973.

69. PaOLucCi L.F., La mutualità nelle cooperative, 1974.

70. De Gennaro A., Crocianesimo e cultura giuridica italiana, 1974.

71. Stortoni L., L'abuso di potere nel diritto penale, 1978.

72. Gianniti F., Prospettive criminologiche e processo penale, 1977.

73. Bonvicini D., Le "joint ventures": tecnica giuridica e prassi societaria, 1977.

74. De Vergottini G., Scritti di storia del diritto italiano, voll. I, II, III, 1977.

75. Lambertini R., I caratteri della Novella 118 di Giustiniano, 1977.

76. DaLla D., L'incapacità sessuale in diritto romano, 1978.

77. Di Pietro A., Lineamenti di una teoria giuridica dell'imposta sull'incremento di valore degli immobili, 1978.

78. Mazzacuva N., La tutela penale del segreto industriale, 1979.

79. Romanelli G., Profilo del noleggio, 1979.

80. Borghesi D., Il contenzioso in materia di eleggibilità, 1979.

81. Dalla Torre G., L'attività assistenziale della Chiesa nell'ordinamento italiano, 1979.

82. CARPI F., La provvisoria esecutorietà della sentenza, 1979.

83. Alleva P., Il campo di applicazione dello statuto dei lavoratori, 1980.

84. Puliatti S., Ricerche sulla legislazione "regionale" di Giustiniano, 1980.

85. FAssò G., Scritti di filosofia del diritto, voll. I, II, III, 1982.

86. Sgubbi F., Uno studio sulla tutela penale del patrimonio, 1980.

87. Lambertini R., Plagium, 1980.

88. Dalla D., Senatus consultum Silanianum, 1980.

89. VANDELLI L., L'ordinamento regionale spagnolo, 1980.

90. NARDi E., L'otre dei parricidi e le bestie incluse, 1980.

91. Pellicanò A., Causa del contratto e circolazione dei beni, 1981.

92. Giardini D., Politica e amministrazione nello Stato fondato sul decentramento, 1981.

93. Bortolotti D., Potere pubblico e ambiente, 1981.

94. Roffi R., Contributo per una teoria delle presunzioni nel diritto amministrativo, 1982.

95. Alessi R., Scritti minori, 1981.

96. Bassanelli Sommariva G., L'imperatore unico creatore ed interprete delle leggi e l'autonomia del giudice nel diritto giustinianeo, 1983.

97. ZanotTi A., Cultura giuridica del Seicento e jus publicum ecclesiasticum nell'opera del cardinal Giovanni Battista De Luca, 1983.

98. Illuminati G., La disciplina processuale delle intercettazioni, 1983.

99. Toniatti R., Costituzione e direzione della politica estera negli Stati Uniti d'America, 1983.

100. NARDi E., Squilibrio e deficienza mentale in diritto romano, 1983. 
101. Dalla D., Praemium emancipationis, 1983.

102. Mazzacuva N., Il disvalore di evento nell'illecito penale - L'illecito commissivo doloso e colposo, 1983.

103. Studi in onore di Tito Carnacini. I. Studi di diritto costituzionale, civile, del lavoro, commerciale, 1983.

104. CAIA G., Stato e autonomie locali nella gestione dell'energia, 1984.

105. Baratti G., Contributo allo studio della sanzione amministrativa, 1984.

106. Вовтоцотті D., Attività preparatoria e funzione amministrativa, 1984.

107. Puliatti S., Ricerche sulle novelle di Giustino II. La legislazione imperiale da Giustiniano I a Giustino II, 1984.

108. Lambertini R., La problematica della commorienza nell'elaborazione giuridica romana, 1984.

109. ZueLLI F., Le collegialità amministrative, 1985.

110. Pedrazzoli M., Democrazia industriale e subordinazione, 1985.

111. Zanotтi M., Profili dogmatici dell'illecito plurisoggettivo, 1985.

112. Ruffolo U., Interessi collettivi o diffusi e tutela del consumatore, I, 1985.

113. Biagi M., Sindacato democrazia e diritto, 1986.

114. Insolera G., Problemi di struttura del concorso di persone nel reato, 1986.

115. Malagù L., Esecuzione forzata e diritto di famiglia, 1986.

116. RICCI G.F., La connessione nel processo esecutivo, 1986.

117. ZanotTi A., Il concordato austriaco del 1855, 1986.

118. Selmini R., Profili di uno studio storico sull'infanticidio, 1987.

119. Dalla D., "Ubi venus mutatur", 1987.

120. Zunarelli S., La nozione di vettore, 1987.

121. ZoLI C., La tutela delle posizioni "strumentali" del lavoratore, 1988.

122. Cavina M., Dottrine giuridiche e strutture sociali padane nella prima età moderna, 1988.

123. CaLIfano L., Innovazione e conformità nel sistema regionale spagnolo, 1988.

124. Sarti N., Gli statuti della società dei notai di Bologna dell'anno 1336 (contributo allo studio di una corporazione cittadina), 1988.

125. SCARPONI S., Riduzione e gestione flessibile del tempo di lavoro, 1988.

126. Bernardini M., Contenuto della proprietà edilizia, 1988.

127. La Torre M., La "lotta contro il diritto soggettivo". Karl Larenz - la dottrina giuridica nazionalsocialista, 1988.

128. Garcia De Enterria J., Le obbligazioni convertibili in azioni, 1989.

129. Biagi Guerini R., Famiglia e Costituzione, 1989.

130. CaIa G., Arbitrati e modelli arbitrali nel diritto amministrativo, 1989.

131. Magagni M., La prestazione caratteristica nella Convenzione di Roma del 19 giugno 1980, 1989.

132. Petroni L., La disciplina pubblicistica dell'innovazione tecnologica in Francia, 1990.

133. Zалотті A., Le manipolazioni genetiche e il diritto della Chiesa, 1990.

134. SARTOR G., Le applicazioni giuridiche dell'intelligenza artificiale, 1990.

135. Rossi L.S., Il "buon funzionamento del mercato comune". Delimitazione dei poteri fra CEE e Stati membri, 1990.

136. Luchetтi G., La legittimazione dei figli naturali nelle fonti tardo imperiali e giustinianee, 1990.

137. SarTi N., Un giurista tra Azzone e Accursio, 1990.

138. Gustapane A., La tutela globale dell'ambiente, 1991.

139. Bottari C., Principi costituzionali e assistenza sanitaria, 1991.

140. Donini M., Illecito e colpevolezza nell'imputazione del reato, 1991.

141. Perulli A., Il potere direttivo dell'imprenditore, 1992.

142. VANDELLI L. (a cura di), Le forme associative tra enti territoriali, 1992.

143. GASPARRI P., Institutiones iuris publici, 1992.

144. CaPUZzo E., Dal nesso asburgico alla sovranità italiana, 1992.

145. Biavati P., Accertamento dei fatti e tecniche probatorie nel processo comunitario, 1992.

146. Ferrari F., Atipicità dell'illecito civile. Una comparazione, 1992.

147. Gustapane A., Sartor G., Verardi C.M., Valutazione di impatto ambientale. Profili normativi e metodologie informatiche, 1992.

148. Orlandi R., Atti e informazioni della autorità amministrativa nel processo penale. Contributo allo studio delle prove extracostituite, 1992.

149. Carpani G., Le aziende degli enti locali. Vigilanza e controlli, 1992. 
150. Musso A., Concorrenza ed integrazione nei contratti di subfornitura industriale, 1993.

151. Donini M., Il delitto contravvenzionale. "Culpa iuris" e oggetto del dolo nei reati a condotta neutra, 1993.

152. Califano Placci L., Le commissioni parlamentari bicamerali nella crisi del bicameralismo italiano, 1993.

153. FoRNASARI G., Il concetto di economia pubblica nel diritto penale. Spunti esegetici e prospettive di riforma, 1994.

154. MANZINI P., L'esclusione della concorrenza nel diritto antitrust italiano, 1994.

155. Ттмотео M., Le successioni nel diritto cinese. Evoluzione storica ed assetto attuale, 1994.

156. Sesta M. (a cura di), Per i cinquant'anni del codice civile, 1994.

157. Tullini P., Contributo alla teoria del licenziamento per giusta causa, 1994.

158. Rescigno F., Disfunzioni e prospettive di riforma del bicameralismo italiano: la camera delle regioni, 1995.

159. Lugaresi N., Le acque pubbliche. Profili dominicali, di tutela, di gestione, 1995.

160. SARTi N., Maximum dirimendarum causarum remedium. Il giuramento di calunnia nella dottrina civilistica dei secoli XI-XIII, 1995.

161. Colliva P., Scritti minori, 1996.

162. Dugato M., Atipicità e funzionalizzazione nell'attività amministrativa per contratti, 1996.

163. GaRdini G., La comunicazione degli atti amministrativi. Uno studio alla luce della legge 7 agosto 1990, n. 241, 1996.

164. Manzini P., I costi ambientali nel diritto internazionale, 1996.

165. Мıттіса M.P., Il divenire dell'ordine. L'interazione normativa nella società omerica, 1996.

166. LuchetTi G., La legislazione imperiale nelle Istituzioni di Giustiniano, 1996.

167. La Torre M., Disavventure del diritto soggettivo. Una vicenda teorica, 1996.

168. Самоn A., Le intercettazioni nel processo penale, 1996.

169. Mancini S., Minoranze autoctone e Stato. Tra composizione dei conflitti e secessione, 1996.

170. Zanobetti Pagnetti A., La non comparizione davanti alla Corte internazionale di giustizia, 1996.

171. Bricola F., Scritti di diritto penale. Vol. I, Dottrine generali, Teoria del reato e sistema sanzionatorio. Vol. II, Parte speciale e legislazione complementare, Diritto penale dell'economia, 1997.

172. Graziosi A., La sentenza di divorzio, 1997.

173. Mantovani M., Il principio di affidamento nella teoria del reato colposo, 1997.

174. Biavati P., Giurisdizione civile, territorio e ordinamento aperto, 1997.

175. Rossi G. (1916-1986), Studi e testi di storia giuridica medievale, a cura di Giovanni Gualandi e Nicoletta Sarti, 1997.

176. Pellegrini S., La litigiosità in Italia. Un'analisi sociologico-giuridica, 1997.

177. Boni G., La rilevanza del diritto dello Stato nell'ordinamento canonico. In particolare la canonizatio legum civilium, 1998.

178. Scritti in onore di Giuseppe Federico Mancini. Vol. I, Diritto del lavoro, 1998.

179. Scritti in onore di Giuseppe Federico Mancini. Vol. II, Diritto dell'Unione europea, 1998

180. Rossi A., Il GEIE nell'ordinamento italiano. Criteri di integrazione della disciplina, 1998.

181. Bongiovanni G., Reine Rechtslehre e dottrina giuridica dello Stato. H. Kelsen e la Costituzione austriaca del 1920, 1998.

182. Caputo G., Scritti minori, 1998.

183. Garrido J.M., Preferenza e proporzionalità nella tutela del credito, 1998.

184. Bellodi Ansaloni A., Ricerche sulla contumacia nelle cognitiones extra ordinem, I, 1998.

185. Franciosi E., Riforme istituzionali e funzioni giurisdizionali nelle Novelle di Giustiniano. Studi su nov. 13 e nov. 80, 1998.

186. Cattabriga C., La Corte di giustizia e il processo decisionale politico comunitario, 1998.

187. Mancini L., Immigrazione musulmana e cultura giuridica. Osservazioni empiriche su due comunità di egiziani, 1998.

188. GustaPane A., L'autonomia e l'indipendenza della magistratura ordinaria nel sistema costituzionale italiano. dagli albori dello Statuto Albertino al crepuscolo della bicamerale, premessa di Giuseppe De Vergottini, 1999.

189. RiCCI G.F., Le prove atipiche, 1999.

190. Canestrari S., Dolo eventuale e colpa cosciente. Ai confini tra dolo e colpa nella struttura delle tipologie delittuose, 1999.

191. FAssò G., La legge della ragione. Ristampa, a cura di Carla Faralli, Enrico Pattaro, Giampaolo Zucchini, 1999. 
192. FAssò G., La democrazia in Grecia. Ristampa, a cura di Carla Faralli, Enrico Pattaro, Giampaolo Zucchini, 1999.

193. Scarciglia R., La motivazione dell'atto amministrativo. Profili ricostruttivi e analisi comparatistica, 1999.

194. Briguglio F., "Fideiussoribus succurri solet", 1999.

195. Maltoni A., Tutela dei consumatori e libera circolazione delle merci nella giurisprudenza della Corte di giustizia, profili costituzionali, prefazione di Augusto Barbera, 1999.

196. Fondaroli D., Illecito penale e riparazione del danno, 1999.

197. Rossi L.S., Le convenzioni fra gli Stati membri dell'Unione europea, 2000.

198. Gragnoli E., Profili dell'interpretazione dei contratti collettivi, 2000.

199. Boni G., La rilevanza del diritto secolare nella disciplina del matrimonio canonico, 2000.

200. Lugaresi N., Internet, privacy e pubblici poteri negli Stati Uniti, 2000.

201. Lalatta Costerbosa M., Ragione e tradizione. Il pensiero giuridico ed etico-politico di Wilehlmvon Humboldt, 2000.

202. Semeraro P., I delitti di millantato credito e traffico di influenza, 2000.

203. VerZa A., La neutralità impossibile. Uno studio sulle teorie liberali contemporanee, 2000.

204. Loldi A., L'atto amministrativo nell'ordinamento democratico. Studio sulla qualificazione giuridica, 2000 .

205. Busetto M.L., Giudice penale e sentenza dichiarativa di fallimento, 2000.

206. Campanella P., Rappresentatività sindacale: fattispecie ed effetti, 2000.

207. Bricola F., Scritti di diritto penale. Opere monografiche, 2000.

208. LASSANDARI A., Il contratto collettivo aziendale e decentrato, 2001.

209. Bianco A., Il finanziamento della politica in Italia, 2001.

210. Raffi A., Sciopero nei servizi pubblici essenziali. Orientamenti della Commissione di garanzia, 2001.

211. Piergigli V., Lingue minoritarie e identità culturali, 2001.

212. Cafaro S., Unione monetaria e coordinamento delle politiche economiche. Il difficile equilibrio tra modelli antagonisti di integrazione europea, 2001.

213. Morrone A., Il custode della ragionevolezza, 2001.

214. Masutti A., La liberalizzazione dei trasporti in Europa. Il caso del trasporto postale, 2002.

215. Zanotti A., Orlando F., L'itinerario canonistico di Giuseppe Caputo, 2002.

216. Lupor M.A., Conflitti transnazionali di giurisdizioni. Vol. I, Policies, metodi, criteri di collegamento. Vol. II, Parallel proceedings, 2002.

217. Lolli A., I limiti soggettivi del giudicato amministrativo. Stabilità del giudicato e difesa del terzo nel processo amministrativo, 2002.

218. Curi F., Tertium datur. Dal Common Law al Civil Law per una scomposizione tripartita dell'elemento soggettivo del reato, 2003.

219. Cottignola G., Studi sul pilotaggio marittimo, 2003.

220. Gardini G., L'imparzialità amministrativa tra indirizzo e gestione. Organizzazione e ruolo della dirigenza pubblica nell'amministrazione contemporanea, 2003.

221. Cevenini C., Virtual enterprises. Legal issues of the on-line collaboration between undertakings, 2003.

222. Monducci J., Diritto della persona e trattamento dei dati particolari, 2003.

223. VIllecco Bettelli A., L'efficacia delle prove informatiche, 2004.

224. Zucconi Galli Fonseca E., La convenzione arbitrale rituale rispetto ai terzi, 2004.

225. BRIGHI R., Norme e conoscenza: dal testo giuridico al metadato, 2004.

226. Luchetti G., Nuove ricerche sulle istituzioni di Giustiniano, 2004.

227. Studi in memoria di Angelo Bonsignori, voll. I, II, 2004.

228. Piperata G., Tipicità e autonomia nei servizi pubblici locali, 2005.

229. Canestrari S., Foffani L. (a cura di), Il diritto penale nella prospettiva europea. Quali politiche criminali per l'Europa? Atti del Convegno organizzato dall'Associazione Franco Bricola (Bologna, 28 febbraio-2 marzo 2002), 2005.

230. Memmo D., Miconi S. (a cura di), Broadcasting regulation: market entry and licensing. Regolamentazione dell'attività radiotelevisiva: accesso al mercato e sistema di licenze. Global Classroom Seminar, 2006.

230.bis Briguglio F., Studi sul procurator, 2007.

231. Querzola L., La tutela anticipatoria fra procedimento cautelare e giudizio di merito, 2006.

232. TARozZ S., Ricerche in tema di registrazione e certificazione del documento nel periodo postclassico, 2006.

233. Вотті F., L'eutanasia in Svizzera, 2007. 
234. Fondaroli D., Le ipotesi speciali di confisca nel sistema penale, 2007.

235. Alagna R., Tipicità e riformulazione del reato, 2007.

236. Giovannini M., Amministrazioni pubbliche e risoluzione alternativa delle controversie, 2007.

237. Montalti M., Orientamento sessuale e costituzione decostruita. Storia comparata di un diritto fondamentale, 2007.

238. Tordini Cagli S., Principio di autodeterminazione e consenso dell'avente diritto, 2008.

239. Legnani Annichini A., La mercanzia di Bologna. Gli statuti del 1436 e le riformagioni quattrocentesche, 2008.

240. LOLLI A., L'amministrazione attraverso strumenti economici, 2008.

241. Vaccarella M., Titolarità e funzione nel regime dei beni civici, 2008.

242. Tubertini C., Pubblica amministrazione e garanzia dei livelli essenziali delle prestazioni, 2008.

243. Fioriglio G., Il diritto alla privacy. Nuove frontiere nell'era di Internet, 2008.

244. Bотті F., Manipolazioni del corpo e mutilazioni genitali femminili, 2009.

245. Nisco A., Controlli sul mercato finanziario e responsabilità penale. Posizioni di garanzia e tutela del risparmio, 2009.

246. Zanobetti Pagnetti A., Il rapporto internazionale di lavoro marittimo, 2008.

247. Mаттіо F., Ricerche sulla formazione della categoria dei cosiddetti quasi delitti, 2010.

248. Bertaccini D., La politica di polizia, 2009.

249. Astrologo A., Le cause di non punibilità. Un percorso tra nuovi orientamenti interpretativi e perenni incertezze dogmatiche, 2009.

250. Di MARIA S., La cancelleria imperiale e $i$ giuristi classici: "Reverentia antiquitatis" e nuove prospettive nella legislazione giustinianea del codice, 2010.

251. VALENTINI E., La domanda cautelare nel sistema delle cautele personali, 2010.

252. Querzola L., Il processo minorile in dimensione europea, 2010.

253. Bologna C., Stato federale e "national interest". Le istanze unitarie nell'esperienza statunitense, 2010.

254. Rasia C., Tutela giudiziale europea e arbitrato, 2010.

255. Zucconi Galli Fonseca E., Pregiudizialità e rinvio (Contributo allo studio dei limiti soggettivi dell'accertamento), 2011.

256. Bellodi Ansaloni A., Ad eruendam veritatem. Profili metodologici e processuali della quaestio per tormenta, 2011.

257. Pontoriero I., Il prestito marittimo in diritto romano, 2011.

258. Giustizia senza confini. Studi offerti a Federico Carpi, 2012.

259. Gustapane A., Il ruolo del pubblico ministero nella Costituzione italiana, 2012.

260. Cataniello M., Premesse per una teoria del pregiudizio effettivo nelle invalidità processuali penali, 2012.

261. Briguglio F., Il Codice Veronese in trasparenza. Genesi e formazione del testo delle Istituzioni di Gaio, 2012 .

262. VAlENTiNi E., La domanda cautelare nel sistema delle cautele personali, Nuova edizione, 2012.

263. TAssinari D., Nemo tenetur se detegere. La libertà dalle autoincriminazioni nella struttura del reato, 2012.

264. Martelloni F., Lavoro coordinato e subordinazione. L'interferenza delle collaborazioni a progetto, 2012.

265. Roversi-Monaco F. (a cura di), Università e riforme. L'organizzazione delle Università degli Studi ed il personale accademico nella legge 30 dicembre 2010, n. 240, 2013.

266. Torre V., La privatizzazione delle fonti di diritto penale, 2013.

267. Raffiotта E.C., Il governo multilivello dell'economia. Studio sulle trasformazioni dello Stato costituzionale in Europa, 2013.

268. Caruso C., La libertà di espressione in azione. Contributo a una teoria costituzionale del discorso pubblico, 2013.

269. Pedrini F., Le "clausole generali". Profili teorici e aspetti costituzionali, 2013.

270. Curi F., Profili penali dello stress lavoro-correlato. L'homo faber nelle organizzazioni complesse, 2013.

271. Casale D., L'idoneità psicofisica del lavoratore pubblico, 2013.

272. Nicodemo S., Le istituzioni della conoscenza nel sistema scolastico, 2013.

273. Legnani Annichini A., «Proxeneta est in tractando». La professione ingrata del mediatore di commercio (secc. XII-XVI), 2013.

274. Monducci J., Il dato genetico tra autodeterminazione informativa e discriminazione genotipica, 2013.

275. Mantovani M., Contributo ad uno studio sul disvalore di azione nel sistema penale vigente, 2014.

276. DE Donno M., Consensualità e interesse pubblico nel governo del territorio, 2015. 
277. PaCilli M., L'abuso dell'appello, 2015.

278. PIšTan Č., Tra democrazia e autoritarismo. Esperienze di giustizia costituzionale nell'Europa centro-orientale e nell'area post-sovietica, 2015.

279. Bellodi Ansaloni A., L'arte dell'avvocato, actor veritatis. Studi di retorica e deontologia forense, 2016.

280. Нохна D., La giustizia criminale napoleonica. A Bologna fra prassi e insegnamento universitario, 2016.

281. Querzola L., L'efficacia dell'attività processuale in un diverso giudizio, 2016.

282. Pieri B., Usurai, ebrei e poteri della Chiesa nei consilia di Paolo da Castro, 2016.

283. Rasia C., La crisi della motivazione nel processo civile, 2016.

284. Drigo C., Le Corti costituzionali tra politica e giurisdizione, 2016.

285. Polacchini F., Doveri costituzionali e principio di solidarietà, 2016.

286. Calcagnile M., Inconferibilità amministrativa e conflitti di interesse nella disciplina dell'accesso alle cariche pubbliche, 2017.

287. VILLA E., La responsabilità solidale come tecnica di tutela del lavoratore, 2017.

288. VINCIERI M., L'integrazione dell'obbligo di sicurezza, 2017.

289. CASALE D., L'automaticità delle prestazioni previdenziali. Tutele, responsabilità e limiti, 2017.

290. GANARIN M., L'interpretazione autentica nelle attuali dinamiche evolutive del diritto canonico, 2018.

291. LaUs F., Il rapporto collaborativo tra pubblico e privato nella contrattazione pubblica. Unione Europea e ordinamenti nazionali: analisi comparata di modelli e riforme, 2018.

292. Bonacini P., Multa scripsit, nihil tamen reperitur. Niccolò Mattarelli giurista a Modena e Padova (1204 ca.-1314 ca.), 2018. 
Finito di stampare nel mese di giugno 2018 per i tipi di Bononia University Press 